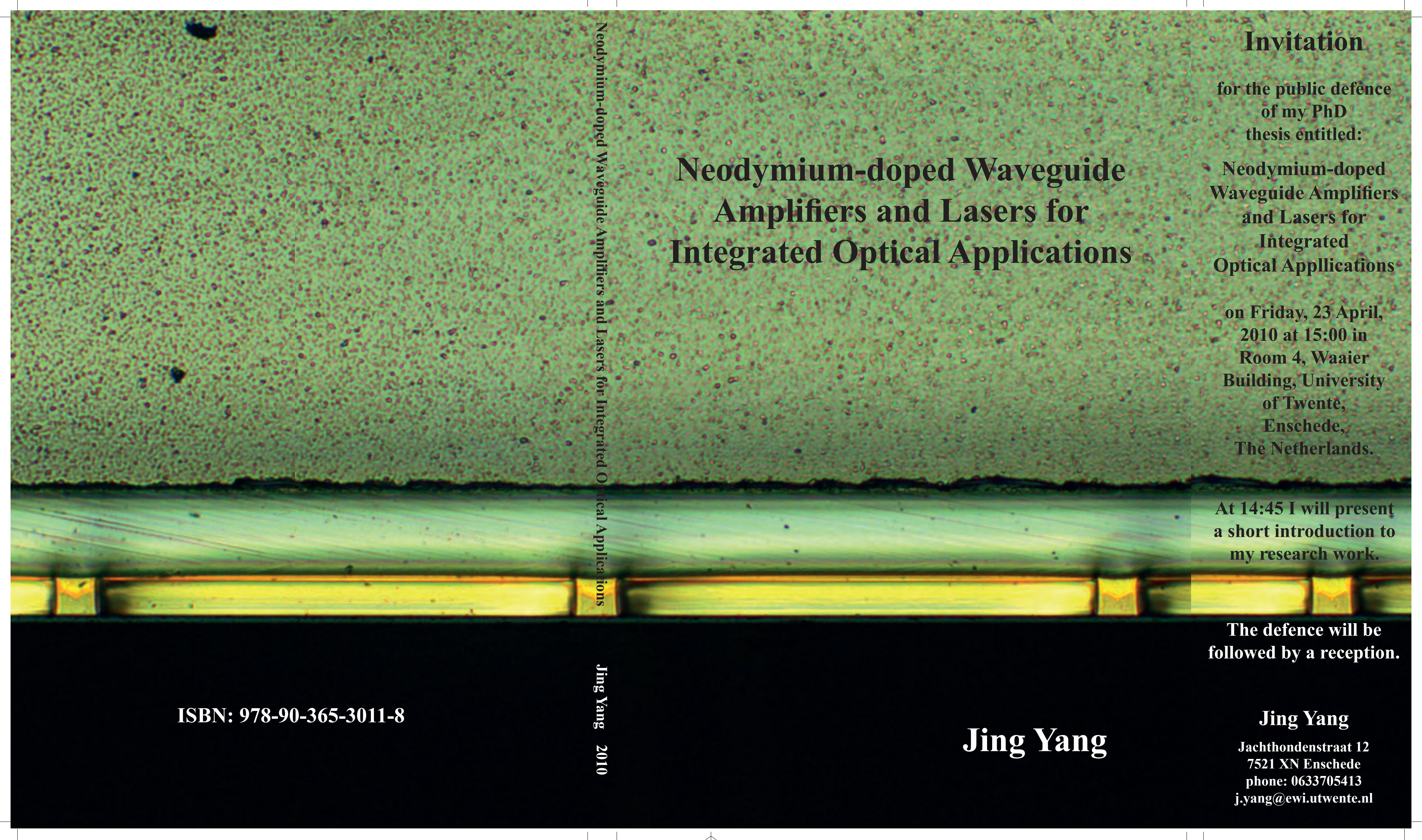




\section{Neodymium-doped Waveguide Amplifiers and Lasers for Integrated Optical Applications}

Jing Yang 


\section{Graduation committee:}

\section{Chairman and Secretary:}

Prof. Dr. Ir. A. J. Mouthaan

University of Twente

\section{Promoters:}

Prof. Dr. M. Pollnau

University of Twente

Prof. Dr. A. Driessen

University of Twente

\section{Members:}

Prof. Dr. F. Laurell

Royal Institute of Technology

Prof. Dr. M. K. Smit

Eindhoven University of Technology

Prof. Dr. W. L. Vos

University of Twente

Dr. M. B. J. Diemeer

Optisense BV

Dr. A. P. Mosk

University of Twente

The research described in this thesis was carried out at the Integrated Optical Micro Systems (IOMS) Group, Faculty of Electrical Engineering, Mathematics and Computer Science, MESA+ Institute for Nanotechnology, University of Twente, P.O. Box 217, 7500 AE Enschede, the Netherlands.

This research was financially supported by the Dutch Technology Foundation STW (project: 06986 “Optical Backplanes”).

Cover design:

Microscope picture of the cross section of neodymium-complex-doped polymer channel waveguides.

ISBN: 978-90-365-3011-8

Printed by IPSKAMP, Enschede, The Netherlands

Copyright (C) 2010 by Jing Yang, Enschede, The Netherlands 


\title{
NEODYMIUM-DOPED WAVEGUIDE AMPLIFIERS AND LASERS FOR INTEGRATED OPTICAL APPLICATIONS
}

\section{DISSERTATION}

\author{
to obtain \\ the degree of doctor at the University of Twente, \\ on the authority of the rector magnificus, \\ prof. dr. H. Brinksma, \\ to be publicly defended \\ on Friday the $23^{\text {rd }}$ of April 2010 at 15.00 \\ by \\ Jing Yang \\ born on the $1^{\text {st }}$ of July 1981 \\ in Hangzhou, Zhejiang, China
}

on account of the decision of the graduation committee, 
This dissertation is approved by:

the promoter: Prof. Dr. M. Pollnau

the promoter: Prof. Dr. A. Driessen 
This thesis is dedicated to my parents,

who have given me everything they could possibly give and to whom there is no way of paying back...

谨以此书献给我的父母， 盛谢他们为我所讨出的一切, 养育之恩，无以回报! 



\section{Contents}

1. Introduction 1

1.1 Integrated Optics 2

1.2 Rare-earth-ion-doped Amplifiers and Lasers 3

1.2.1 Rare-earth Ions $\quad 3$

1.2.2 Host Materials $\quad 4$

$1.3 \mathrm{Nd}^{3+}$-doped Active Waveguide Devices 4

1.3.1 $\mathrm{Nd}^{3+}$ ions 4

1.3.2 $\mathrm{Nd}^{3+}$-doped Polymer Waveguides $\quad 6$

$\begin{array}{lll}\text { 1.3.3 } & \mathrm{Al}_{2} \mathrm{O}_{3}: \mathrm{Nd}^{3+} \text { Waveguides } & 8\end{array}$

1.4 Amplification for Applications in Optical Backplanes 8

$\begin{array}{lll}1.5 & \text { Outline } & 9\end{array}$

2. Optical Processes in $\mathrm{Nd}^{3+}$ Ions $\quad 11$

$\begin{array}{ll}2.1 \text { Introduction } & 12\end{array}$

2.2 Interaction between Light and Atomic Systems in Active Media 12

2.2.1 Einstein Coefficients $\quad 12$

2.2.2 Absorption, Emission, and Gain 14

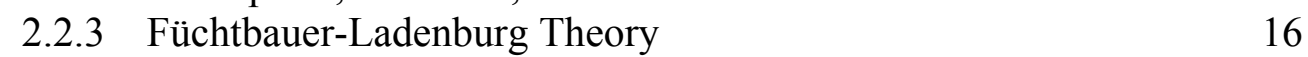

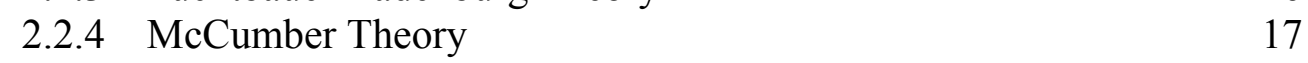

$\begin{array}{lll}2.2 .5 & \text { Judd-Ofelt Theory } & 18\end{array}$

$\begin{array}{ll}2.3 \text { Amplification and Lasing in } \mathrm{Nd}^{3+} & 19\end{array}$

$\begin{array}{lll}2.3 .1 & \text { Energy Transitions of } \mathrm{Nd}^{3+} \text { Ions } & 19\end{array}$

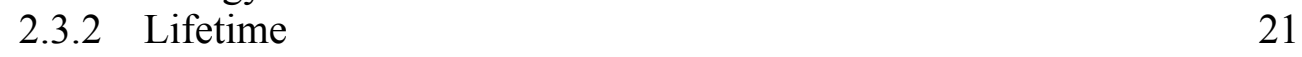

2.3.3 Energy-transfer Upconversion $\quad 21$

2.3.4 Amplified Spontaneous Emission $\quad 22$

2.3.5 Rate Equations $\quad 22$

$\begin{array}{lll}2.4 & \text { Summary } & 25\end{array}$

3. $\mathrm{Nd}^{3+}$-doped Waveguides: Fabrication and Optical Characterization 27

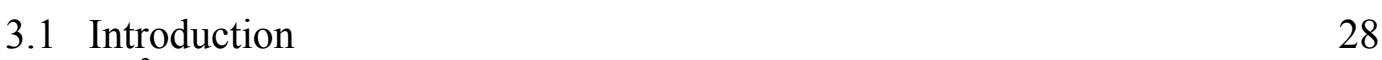

$3.2 \mathrm{Nd}^{3+}$-complex-doped Polymer Waveguide Fabrication 28

$\begin{array}{ll}\text { 3.2.1 Film Deposition } & 28\end{array}$

$\begin{array}{ll}\text { 3.2.2 Waveguide Structuring } & 31\end{array}$

$\begin{array}{lll}3.3 & \mathrm{Al}_{2} \mathrm{O}_{3}: \mathrm{Nd}^{3+} \text { Waveguide Fabrication } & 36\end{array}$

3.3.1 Film Deposition $\quad 36$

$\begin{array}{lll}\text { 3.3.2 Waveguide Structuring } & 38\end{array}$

$\begin{array}{ll}3.4 & \text { Optical Losses }\end{array}$

3.4.1 Optical Attenuation and Loss Measurement $\quad 40$

3.4.2 Loss and Absorption Spectra 43

3.4.3 Judd-Ofelt Analysis 49

3.4.4 Determination of $\mathrm{Nd}^{3+}$ Concentration in Host Materials 51 
3.5 Luminescence and Lifetime Measurements 52

3.5.1 Luminescence Spectra $\quad 52$

3.5.2 Luminescence Lifetime $\quad 55$

$\begin{array}{ll}3.6 & \text { Summary } \\ & 60\end{array}$

4. $\mathrm{Nd}^{3+}$-doped Waveguide Amplifiers

$\begin{array}{lll}4.1 & \text { Introduction } & 62\end{array}$

4.2 Optical Gain Investigation 63

$\begin{array}{ll}4.3 \text { Optical Gain at } 1064 \mathrm{~nm} & 66\end{array}$

4.4 Optical Gain at $1330 \mathrm{~nm} \quad 69$

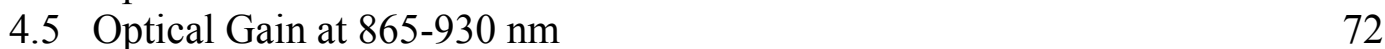

4.6 Energy-transfer Upconversion Parameters $\quad 81$

$\begin{array}{lll}4.7 & \text { Summary } & 85\end{array}$

$\begin{array}{lr}\text { 5. } \mathrm{Nd}^{3+} \text {-doped Polymer Waveguide Lasers } & 87\end{array}$

$\begin{array}{llr}5.1 & \text { Introduction } & 88\end{array}$

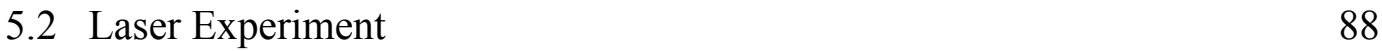

5.3 Laser Results $\quad 89$

$\begin{array}{lll}5.4 \text { Summary } & 96\end{array}$

6. Waveguide Amplifiers Integrated in Optical Backplanes 99

6.1 Introduction 100

6.2 Polymer Waveguides Embedded in Optical Backplanes 101

$6.3 \quad \mathrm{Al}_{2} \mathrm{O}_{3}: \mathrm{Nd}^{3+}$ Large-core Waveguide Amplifiers $\quad 102$

6.3.1 Motivation and Waveguide Geometries 102

6.3.2 Investigation of Waveguide Amplifiers 104

6.4 Design of $\mathrm{Nd}^{3+}$-doped Waveguide Amplifiers Integrated in Optical 106 Backplanes

6.4.1 $\mathrm{Al}_{2} \mathrm{O}_{3}: \mathrm{Nd}^{3+}$ Waveguides Integrated with Polymer 106

Waveguides

6.4.2 Waveguide Tapers in $\mathrm{Al}_{2} \mathrm{O}_{3}: \mathrm{Nd}^{3+} \quad 109$

$\begin{array}{ll}\text { 6.4.3 Side Pumping Coupler } & 110\end{array}$

6.5 Demonstration of Waveguide Amplifiers Integrated in Optical 112

Backplanes

6.5.1 Gain Measurement 112

6.5.2 Gain Results 113

$\begin{array}{ll}6.6 \text { Summary } & 115\end{array}$

$\begin{array}{lr}\text { 7. Conclusions } & 117\end{array}$

$\begin{array}{lr}\text { References } & 121\end{array}$

$\begin{array}{ll}\text { Acknowledgements } & 131\end{array}$

$\begin{array}{ll}\text { List of Publications } & 133\end{array}$ 


\section{Chapter 1}

\section{Introduction}

In this Chapter an introduction to integrated optics is presented. Rare-earthion-doped waveguides and, in particular, $\mathrm{Nd}^{3+}$-doped waveguides are used as active devices such as amplifiers and lasers. The advantages of polymers and $\mathrm{Al}_{2} \mathrm{O}_{3}$ as host materials are discussed. One of their attractive applications and the final aim of this work is optical waveguide amplification in optical backplanes. Finally, the layout of this thesis is presented. 


\subsection{Integrated Optics}

The main aim of integrated optics is developing miniaturized optical devices of high functionality on a common substrate to reduce the size and cost of optical systems. Over the last few decades, there has been a continuous expansion in the field of integrated optics along-side the growth of fiber-optic communications.

There are various applications for integrated optics. Interconnection by optics is an ideal solution for the speed limit in Print Circuit Board (PCB) backplanes [1-4]. Besides short-distance signal transmission, there are also potential applications of integrated optics in telecommunication components including those facilitating fiber-to-the homes [5-8]. Furthermore, integrated optical sensors, imaging devices, and laser sources are promising for food safety, medical diagnostics, and biological warfare [9-13].

A basic element of a photonic integrated circuit is the planar optical waveguide, which is usually fabricated on a substrate with thickness of $\sim 0.5-1 \mathrm{~mm}$. The most commonly used ones are silicon substrates. An optical waveguide consists of a high refractive index guiding layer, sandwiched between two lower-index cladding layers. The optical signal travels in the guiding layer and is confined in the transverse directions. The propagating light in slab waveguides is confined only in one direction, while in channel waveguides it is confined in both horizontal and vertical directions. In contrast to optical fibers, which typically have a core with a radius of $9 \mu \mathrm{m}$ and a refractive index contrast between the core and cladding layers is about $\Delta n=0.003$ for single-mode guiding, planar channel waveguides can have core sizes as small as a few hundreds of nanometers and index contrasts of up to 2.5 . The difference between refractive index of the core and cladding of channel waveguides determines the minimum bend radius, thus the size of devices on the chip.

A key consideration in the design and realization optical waveguides and integrated optical systems is the choice of materials. There are several requirements for a good waveguide material, including low optical loss, ease of fabrication and reproducibility, and long-time stability. Various materials have been investigated and applied to form optical waveguides. Traditionally, fine optical devices have been made of inorganic materials, such as crystals, glasses, silicon, III-V semiconductors and dielectric materials, with high thermal stability, long-term reliability, and low loss. With a broad range of property adjustability and multiple choices of processing approaches, polymers have been emerging as very promising light transmission media for integrated optics as well as fiber optical telecommunication.

According to the functions, the multiple components included in an optical circuit can be divided into passive and active devices. To manipulate the optical signals on a local scale, passive devices like splitters, couplers, multiplexers, and demultiplexers are used, while optical sources for providing light, amplifiers for compensating signal loss, and modulators for converting energy between light and electricity are so-called active devices.

Optical losses in waveguides, coupling losses, or intrinsic intensity losses in optical splitters, call for the integration of optical amplifiers with waveguides and other components in optical integrated circuit. Besides, integrated waveguide laser sources are also attractive in many integrated systems. One simple and efficient way to achieve optical gain in waveguides is doping the guiding layer with an active element which generates light when pumping it properly. 
In this thesis, polymer and amorphous aluminum oxide waveguides doped with $\mathrm{Nd}^{3+}$ ions are investigated as active materials for waveguide amplification and lasing. In the next two sections, more details of rare-earth ions, host materials, $\mathrm{Nd}^{3+}$ doped amplifiers and lasers, and the advantages of two different material systems will be presented. In Section 1.4, application of $\mathrm{Nd}^{3+}$-doped waveguide amplifiers in integrated optics, specifically optical amplification for use in optical backplanes, will be discussed.

\subsection{Rare-earth-ion-doped Amplifiers and Lasers}

\subsubsection{Rare-earth Ions}

Rare-earth elements [14] include elements 57-71 (the lanthanides) and 89-103 (the actinides) of the periodic table, such as erbium, neodymium, europium, and are well known for the special luminescence properties of their trivalent ions. Their optical properties result from the fact that the electrons of the partially filled $4 f$ shell are shielded from the surrounding completely filled $5 s$ and $5 p$ shells. The energy levels of the $4 f$ shell have equal parity, and hence electric dipole transitions are forbidden. In a solid, the small mixing with odd-parity wave functions makes the transition allowed. When optically pumped, exciting the ions to a higher energy level, a large number of transitions are possible, producing optical emission at characteristic wavelengths. The influence of the local electric field around the ion in a solid environment removes the degeneracy of the $4 f$ levels, resulting in a Stark splitting of the energy levels. The emission wavelengths of rare-earth ions are almost independent of the host material.

Study of the rare-earth ions started from the early 1900s, and rare-earth-iondoped amplifiers and lasers have been theoretically and experimentally studied since then [14-16]. Several of the rare-earth ions have been applied extensively in amplifiers and lasers, and Table 1.1 gives the luminescence wavelength peaks of the most commonly used transitions in prominent rare-earth ions. Although the emission wavelength is mainly dependent on the rare-earth ion, there is some slight shift of the emission peak values and difference of emission bandwidth due to the host materials.

Table 1.1. Luminescence wavelengths of rare-earth ions.

\begin{tabular}{cc}
\hline \hline Rare-earth ion & Luminescence wavelength \\
\hline $\mathrm{Nd}^{3+}$ & $880,1060,1330 \mathrm{~nm}$ \\
$\mathrm{Eu}^{3+}$ & $615 \mathrm{~nm}$ \\
$\mathrm{Ho}^{3+}$ & $2100 \mathrm{~nm}$ \\
$\mathrm{Er}^{3+}$ & $1550 \mathrm{~nm}$ \\
$\mathrm{Tm}^{3+}$ & $1900 \mathrm{~nm}$ \\
$\mathrm{Yb}^{3+}$ & $1020 \mathrm{~nm}$ \\
\hline
\end{tabular}

The Nd:YAG laser at $1064 \mathrm{~nm}$, first demonstrated by Geusic et. al at Bell Laboratories in 1964 [17], is one of the most commonly used laser. For telecommunication applications, $\mathrm{Er}^{3+}$ and $\mathrm{Nd}^{3+}$ are used as they have transitions at $1530 \mathrm{~nm}$ and $1340 \mathrm{~nm}$, respectively, which are two standard wavelengths for telecommunication. The rare-earth-ion-doped optical fiber amplifier was invented by H. J. Shaw and Michel Digonnet in the early 1980s, and the Er-doped fiber amplifier (EDFA) $[18,19]$ enables the most widely influential application of rare-earth ions in 
optical fiber communications. Fiber amplifiers and lasers with various rare-earth dopants, such as $\mathrm{Eu}^{3+}, \mathrm{Yb}^{3+}, \mathrm{Nd}^{3+}$, have also been widely studied and used [16, 20-26]. Furthermore, to provide light sources and amplify optical signals, rare-earth-iondoped waveguide amplifiers and lasers have attracted considerable interest and have been reported in various host materials [27-47].

\subsubsection{Host Materials}

Various host materials have been investigated and reported for rare-earth-ion dopants in integrated optical applications. Along the general requirements for optical waveguide materials in integrated optics, there are several requirements which must be met for a good host material, including availability of suitable rare-earth-ion bonding sites, high solubility of ions without clustering, a sufficiently low phonon energy to prevent non-radiative decay and long radiative lifetimes of excited states to provide efficient luminescence transitions.

Crystals, glasses, and polymers are three main groups of host materials that have been used in rare-earth-ion-doped systems. Each type has its specific advantages and disadvantages. Crystals have high stability and sharp emission cross sections for excellent laser performance [15, 27-29, 34-37, 44]. However, the bandwidth and tunable wavelength range of amplifiers and lasers are limited by the narrow wavelength range of the peaks. Besides, special growth and structuring facilities are required for waveguide realization, and these materials cannot be integrated with other materials platforms due to the requirement of crystalline lattice matching. Polymers are of interest due to their low cost, ease of fabrication, straight-forward integration with other materials, and broad emission spectra [30-33, 38, 39, 49]. The drawbacks of such materials are the thermal stability and the additional absorption color centers. Glass materials, on the other hand, exhibit a broad emission spectrum and rather high thermal stability and durability. Various types of glasses have been investigated for optical gain media [45-48], but most of them have low refractive index contrasts which limit the integration density.

\section{$1.3 \mathrm{Nd}^{3+}$-doped Active Waveguide Devices}

\subsubsection{Nd ${ }^{3+}$ Ions}

Neodymium is a widely used rare-earth ion for optical amplifications and lasing. Neodymium-doped lasers [27-29] and amplifiers [28, 30-33] are of interest for applications at the ion's specific emission wavelengths and have been widely studied. Figures 1.1(a) and (b) display the absorption and luminescence spectra, respectively, of $\mathrm{Nd}^{3+}$ in a phosphate glass host [48]. There are five major absorption bands of $\mathrm{Nd}^{3+}$ in the visible and near infrared wavelength range, which are $520 \mathrm{~nm}, 580 \mathrm{~nm}, 745 \mathrm{~nm}$, $800 \mathrm{~nm}$, and $880 \mathrm{~nm}$. The three luminescence peaks around $880 \mathrm{~nm}, 1060 \mathrm{~nm}$, and $1330 \mathrm{~nm}$ correspond to the transitions ${ }^{4} F_{3 / 2} \rightarrow{ }^{4} \mathrm{I}_{9 / 2},{ }^{4} F_{3 / 2} \rightarrow{ }^{4} \mathrm{I}_{11 / 2}$, and ${ }^{4} F_{3 / 2} \rightarrow{ }^{4} \mathrm{I}_{13 / 2}$, respectively. The spectra of absorption and luminescence are much wider and than in crystalline hosts [15]. 


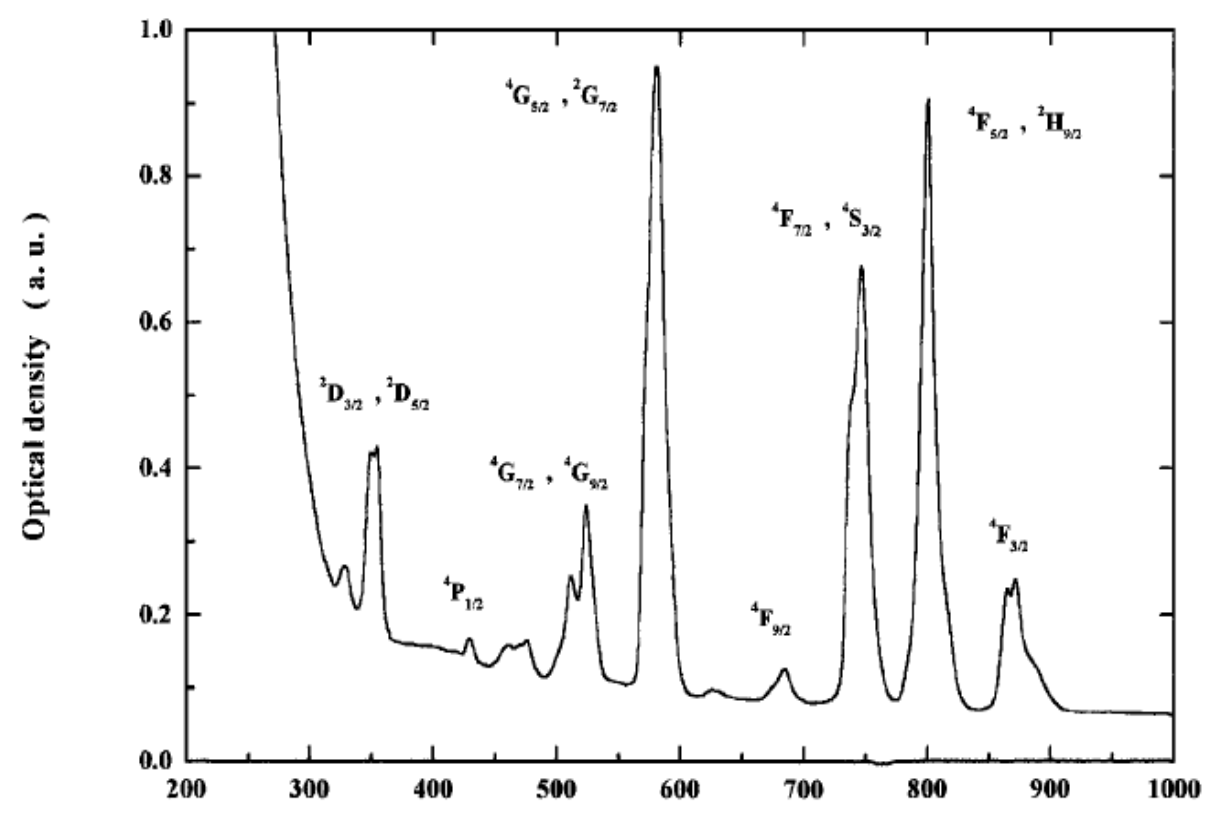

Wavelength ( $\mathrm{nm})$

(a)

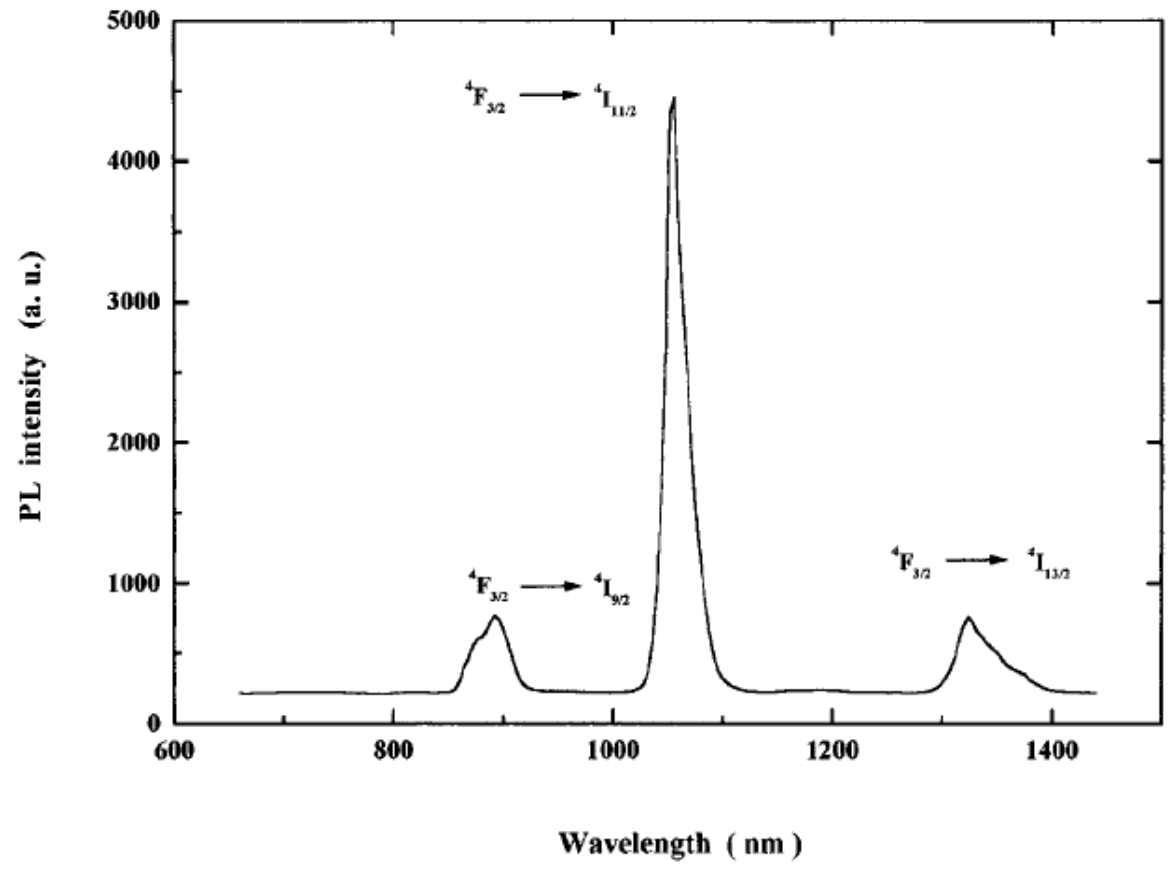

(b)

Fig. 1.1. (a) Absorption and (b) luminescence spectrum of $\mathrm{Nd}^{3+}$ in a phosphate glass.

The $\mathrm{Nd}^{3+}$ luminescence transition at approximately $1060 \mathrm{~nm}$ involves a fourlevel scheme with fast multiphonon transitions populating the upper and depleting the lower laser level. Its large stimulated emission cross section allows a low laser threshold. Nd-doped crystal and glass amplifiers and lasers at $1060 \mathrm{~nm}$ have been widely studied and reported in both bulk materials as well as waveguide structures $[15$, 17, 27-29].

The $\mathrm{Nd}^{3+}$ transition ${ }^{4} \mathrm{~F}_{3 / 2} \rightarrow{ }^{4} \mathrm{I}_{13 / 2}$ around $1330 \mathrm{~nm}$, corresponding to the 
wavelength of the second standard telecommunication window, is interesting for amplification of optical signal in integrated devices and systems at the telecommunication O-band (1260-1360 nm).

Furthermore, the emission on the ${ }^{4} \mathrm{~F}_{3 / 2} \rightarrow{ }^{4} \mathrm{I}_{9 / 2}$ ground-state transition around $865-930 \mathrm{~nm}$ is of interest for signal amplification in integrated optical applications, e.g. data transmission in optical interconnects and medical diagnostics. However, the efficiency at this wavelength range is not as large as that of the other two transitions due to the re-absorption by $\mathrm{Nd}^{3+}$ ions in their ground state.

\subsection{2 $\mathrm{Nd}^{3+}$-doped Polymer Waveguides}

Polymers are promising host candidates for integrated optical devices due to their low cost, ease of fabrication, and potential combination with many substrate or layer materials.

Most commonly developed polymers for use in integrated optical applications include acrylates, polyimides, and olefins [49]. The refractive index of these polymers can be tuned with an accuracy of less than 0.0001 by blending and copolymerizations [50].

Many techniques have been used to fabricate polymer waveguides and devices. Photolithography followed by reactive ion etch (RIE) is a common process for polymer waveguide fabrication and the complexity of the process is similar to those used for glass devices. Simple direct writing processes, such as UV lithography and electron beam lithography [51] are advantageous over other techniques because fewer steps are involved. Using one-step electron beam direct writing, nanometer patterns with flexibility in writing complex structures are also possible. Microfabrication using non-photolithographic techniques has also been used in fabrication of perfluorocyclobutane (PFCB) polymer waveguides [49]. Furthermore, photodefinition is a simple and straightforward way to realize polymer waveguides [52], as only standard processes, e.g. spin-coating and standard lithography, are required.

Low optical loss is one of the major requirements for waveguide materials. The waveguide propagation loss of polymer structures can be below $0.1 \mathrm{~dB} / \mathrm{cm}[30$, 51], which is well acceptable for integrated optical devices with $\mathrm{cm}$ length scales. The optical loss in polymer waveguides is mainly caused by side-wall and top-wall roughness, scattering from polymer particles, and material absorption from fundamental and overtone vibrations of molecular bonds. The most important absorption at the infrared wavelength range is due to vibrational states of $\mathrm{O}-\mathrm{H}$ and $\mathrm{C}$ $\mathrm{H}$ bonds. It can be reduced by substitution of the hydrogen atoms by deuterium, fluorine, or chlorine.

Low-loss polymer passive waveguides for many integrated optical applications, such as $1 \times \mathrm{N}$ splitters [49], directional couplers [49], arrayed-waveguide grating (AWG) multiplexers [49, 53], and embedded waveguides in optical backplanes [2-4], have been realized. High speed modulation has also been demonstrated in tailored electro-optic polymer materials [54], showing that fast electronic response in polymer materials is possible.

Optical amplification in rare-earth-ion-doped polymers is also an interesting and important research area. However, rare-earth ions are insoluble in polymers. This 
makes the fabrication of rare-earth-ion-doped polymer waveguides more complicated than that of inorganic waveguides. This problem can be solved using a variety of techniques. One simple way is to encapsulate the rare-earth ion into an organic complex, making it dissolvable in a polymer matrix.

Recent work [30] on polymer-based, rare-earth-ion-doped planar optical waveguides has resulted in the demonstration of optical gain, e.g. in neodymiumdoped [31-33] and erbium-doped [38, 39] polymer waveguide amplifiers. In Refs. [31] and [32], $\mathrm{Nd}^{3+}$-doped photolime gel polymer planar waveguides were demonstrated with a maximum signal enhancement of $8.5 \mathrm{~dB}$. However, the photolime gel polymer contains a significant amount of $\mathrm{O}-\mathrm{H}$ groups, which leads to a decrease in the signal output, and the device does not possess long-term stability. In Ref. [33], after a highly complex fabrication procedure involving a multi-layer architecture, a signal enhancement of $8 \mathrm{~dB}$ at $1060 \mathrm{~nm}$ was obtained in a $\mathrm{NdCl}_{3}$-doped polymer channel waveguide. A challenge for the realization of polymer -based $\mathrm{Nd}^{3+}$-doped channel waveguide amplifiers is to exploit the potential simplicity of polymer deposition and micro-structuring.

To date laser action has been achieved in many optically pumped solid-state waveguides based on dye-doped polymers [55] and highly luminescent semiconducting polymers [56], including conjugated polymers, dendrimers, and spirocompounds. An attractive feature of these lasers is that they naturally form a four-level laser system and, as their $\pi-\pi^{*}$ transitions are allowed, most of them exhibit extremely high absorption and stimulated-emission cross-sections, attributes that can be exploited for lasing. However, the downside of these lasers is that they suffer from the accumulation of triplet excitons produced through intersystem crossing, which are responsible for the excited-state triplet absorption. The latter is due to its spectral overlap with the stimulated emission and the long lifetime of the triplet state limits the prospects of continuous-wave lasing. For this reason pumping of these types of polymer lasers is performed with pulsed lasers operating at repetition rates sufficiently low (between $10 \mathrm{~Hz}$ and $10 \mathrm{kHz}$ ) to allow dissipation of the triplet populations between the pulses. In addition, the pulse widths of the excitation sources are typically in the range between $100 \mathrm{fs}$ to $10 \mathrm{~ns}$ in order to provide the gain medium with sufficient time to recover between successive pump pulses [56]. For solid-state dye lasers, the only report to date is on quasi-continuous-wave laser operation involving a dye-doped polymer and was achieved using a disk-shaped active medium, which was rotated at a high speed to resemble the circulating flow of a liquid dye laser that continuously refreshes the chromophores that are exposed to the excitation beam. This laser, however, is impractical for device integration and has delivered a rather noisy output [57]. Laser emission from rare-earth-ion-doped polymer systems was reported for $\mathrm{Nd}^{3+}$-doped chelates embedded in a PMMA host, where heavily dumped relaxation oscillations were observed under excitation with an arc-length flash tube [58]. In a more recent report transient laser emission was obtained from an $\mathrm{Eu}^{3+}$ complex-doped polymer by pumping with an ultraviolet $\mathrm{N}_{2}$ pulsed laser [59]. Although there are several reports on optical waveguide amplifiers based on various polymer materials doped with rare-earth ions such as $\mathrm{Nd}^{3+}, \mathrm{Er}^{3+}, \mathrm{Eu}^{3+}$, and $\mathrm{Er} / \mathrm{Yb}^{3+}$, a rare-earth-ion-doped polymer waveguide laser has as yet not been demonstrated. Besides, the continuous wave (CW) laser operation is another challenging aim in polymer materials due to the low damage threshold compared to that of inorganic materials. Therefore, polymer waveguide materials with good thermal stability are required and are of interest for research. 


\subsection{3 $\mathrm{Al}_{2} \mathrm{O}_{3}: \mathrm{Nd}^{3+}$ Waveguides}

Amorphous $\mathrm{Al}_{2} \mathrm{O}_{3}$ is also a promising host for rare-earth ions and an attractive waveguide material. It has been studied and demonstrated to be a good material for passive integrated optics due to low losses and high transparency over a wide wavelength range. It has a higher refractive index contrast in comparison to other glass hosts, allowing smaller waveguide bend radii [60]. Furthermore, the material acts as an excellent host for rare-earth dopants, because the rare-earth ions are wellmatched to the oxygen bonding sites [61], and high solubility of rare-earth ions is possible. In addition, the amorphous nature allows broadband emission bands of rareearth ions. Compared with polymers, $\mathrm{Al}_{2} \mathrm{O}_{3}$ has better thermal stability. In addition, $\mathrm{Al}_{2} \mathrm{O}_{3}$ has better integration potential for active devices due to its higher index contrast.

$\mathrm{Al}_{2} \mathrm{O}_{3}: \mathrm{Er}^{3+}$ waveguide amplifiers have been reported by several research groups [40-42, 62-66]. A maximum internal net gain of $2 \mathrm{~dB} / \mathrm{cm}$ at $1533 \mathrm{~nm}$ by pumping at $977 \mathrm{~nm}$ [40], and a net gain of up to $11 \mathrm{~dB}$ at $1532 \mathrm{~nm}$ with data transmission at a rate of $170 \mathrm{Gbit} / \mathrm{s}$ [42] have been demonstrated. These results indicate that $\mathrm{Al}_{2} \mathrm{O}_{3}$ is well suited as a host for rare-earth ions. However, so far most of the work on $\mathrm{Al}_{2} \mathrm{O}_{3}$ active waveguide devices has been concentrated on $\mathrm{Er}^{3+}$ ions at the telecommunication wavelength near $1550 \mathrm{~nm}$. Study on $\mathrm{Al}_{2} \mathrm{O}_{3}: \mathrm{Nd}^{3+}$ waveguide amplifiers and integrated lasers is therefore promising.

\subsection{Amplification for Applications in Optical Backplanes}

Interconnect between cards over their PCB backplane has become a bottleneck in high-end systems like servers, telecom switches, and advanced radar systems as a result of the continuous increase of the microprocessor clock rate, in addition to the continuous increase of data transmission rates [67]. Solutions in the form of hybrid electro-optical interconnect systems have been proposed [68, 69], as well as a reevaluation of the frontiers of electrical interconnects [70]. While there are applications with a need for high bandwidth, nontrivial electromagnetic interference (EMI) problems must be solved [71]. These problems are related to the high-density packaging of copper conductors and the fact that these can act as antennas, thereby receiving and transmitting electromagnetic radiation. Besides, the increased signal attenuation and dispersion at high frequencies must also be tackled [72].

Use of optical waveguides in optical backplanes and motherboards is one possible solution (Fig. 1.2), because these are far less sensitive to EMI than electrical interconnects [71]. Polymer materials are promising as waveguide materials in this application due to the low cost, ease of fabrication, and rather high packaging density. There has been a significant worldwide research interest over the past 15 years on optical chip interconnects, optical motherboards and optical backplanes, and optical interconnections based on embedded polymer waveguides have recently been reported [2-4].

In the "Optical Backplanes" project, we proposed to develop PCB backplanes with an embedded polymeric optical waveguide layer, which was realized with PCB compatible, low-cost fabrication techniques for the transmission (over 0.5 $\mathrm{m})$ of broadband (2.5 Gbps/channel) signals. 


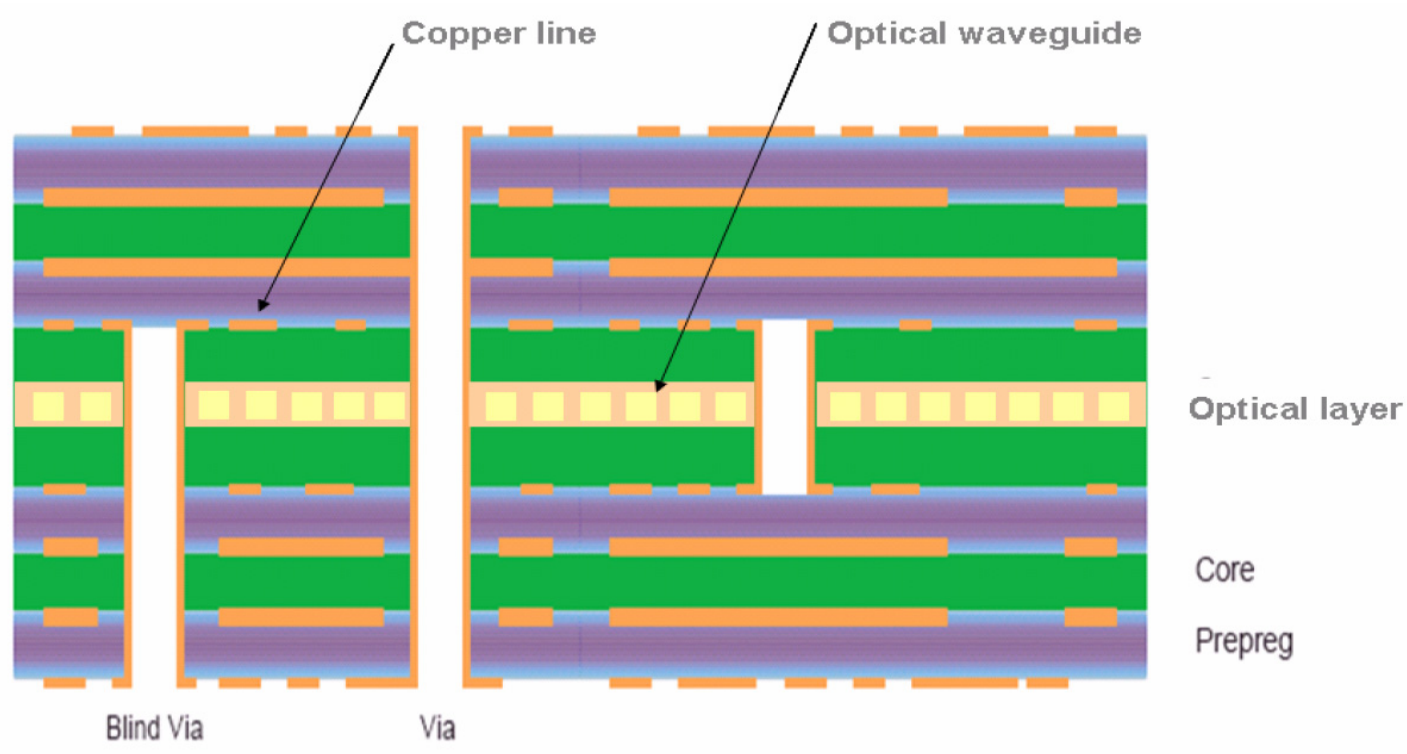

Fig. 1.2. Backplane PCB with embedded optical waveguides: Optical Backplane

However, investigations on the optical power budget for realistic polymerwaveguide based high-speed links via optical backplanes showed that coupling and routing dependent losses reduce the optical power to a significant extent [1]. Therefore, additional signal amplification within the board is necessary to compensate optical losses due to waveguide materials, signal routing, and input/output coupling. Integration of amplifying materials into optical backplanes is one efficient solution.

$850 \mathrm{~nm}$ is chosen for optical interconnects in printed circuit boards (PCBs) [2-4] due to the maturity of VCSEL technology at this wavelength. The emission of $\mathrm{Nd}^{3+}$ on the ${ }^{4} \mathrm{~F}_{3 / 2} \rightarrow{ }^{4} \mathrm{I}_{9 / 2}$ ground-state transition is close to this wavelength, and optical amplification based on rare-earth-ion-doped waveguides integrated in optical backplane systems is therefore attractive. Investigation of the feasibility of optical amplifiers for optical backplanes using rare-earth-ion-doped waveguide amplifiers is one of the aims of this work.

\subsection{Outline}

This thesis mainly focuses on the study of $\mathrm{Nd}^{3+}$-doped waveguide amplifiers and lasers for integrated optical applications. Two types of waveguide materials are investigated and corresponding waveguide amplifiers and lasers are fabricated and characterized.

In Chapter 2 a background theory of the interaction of light and matter in active media based on Einstein's treatment is presented. The main processes and parameters for optical amplifiers and lasers in neodymium-doped systems, including the absorption, emission, and gain cross section, lifetime, energy-transfer upconversion (ETU), and amplified spontaneous emission (ASE) are discussed. The rate equations of $\mathrm{Nd}^{3+}$ are explained and simplified for the simulation of optical gain in $\mathrm{Nd}^{3+}$-doped materials. 
In Chapter 3 the different fabrication techniques to realize $\mathrm{Nd}^{3+}$-complexdoped polymer waveguides and $\mathrm{Al}_{2} \mathrm{O}_{3}: \mathrm{Nd}^{3+}$ waveguides are presented. The optical properties of the two types of waveguides, including optical loss, luminescence spectrum, and luminescence lifetime are investigated and discussed.

In Chapter 4 optical gain is demonstrated for waveguide amplifiers at each of the three main $\mathrm{Nd}^{3+}$ transitions for the polymer and $\mathrm{Al}_{2} \mathrm{O}_{3}$-based materials. The results are compared with those from the theoretical study.

In Chapter $5 \mathrm{CW}$ laser operation achieved in $\mathrm{Nd}^{3+}$-complex-doped polymer waveguide lasers is presented, which a promising application of the polymer amplifiers discussed in Chapter 4.

In Chapter 6 other application of $\mathrm{Nd}^{3+}$-doped waveguide amplifiers is demonstrated. Optical waveguide amplifiers based on $\mathrm{Al}_{2} \mathrm{O}_{3}: \mathrm{Nd}^{3+}$ used in optical backplanes are discussed and demonstrated. are outlined.

In Chapter 7 general conclusions based on the work presented in this thesis 


\section{Chapter 2}

\section{Optical Processes in $\mathrm{Nd}^{3+}$ Ions}

In this Chapter, a background theory of the interaction of light and matter in active media based on Einstein's treatment is presented. The main processes and parameters for optical amplifiers and lasers in neodymium-doped systems, including the absorption, emission, and gain cross section, lifetime, energytransfer upconversion (ETU), and amplified spontaneous emission (ASE) are discussed. The rate equations of $\mathrm{Nd}^{3+}$ are explained and simplified for simulation of optical in $\mathrm{Nd}^{3+}$-doped materials. 


\subsection{Introduction}

Rare-earth ions are well known as gain media for optical amplification and lasing. Using an optical pump beam, the ions are pumped into an excited state. Once a signal photon passes by, the energy of the excited state can be released by emission of another photon with the same properties, i.e. same wavelength, direction, and phase, as the signal photon. By this way, signal light can be amplified. Furthermore, by introducing a resonant cavity as well as pumping above the threshold, laser operation can be achieved. Eleven out of fourteen rare-earth ions have been commonly used for amplification and lasing. In this work, we will focus on the study of neodymium ions in their trivalent oxidation state.

This Chapter contains the basic information, principles and concepts necessary for proper understanding of the physical phenomena important for $\mathrm{Nd}^{3+}-$ doped materials. As a starting point, a brief review of Einstein's treatment is presented, describing the interaction of light and matter in active media. The relationship among absorption, emission, and gain will be discussed, and the background spectroscopic theory related to these phenomena will be provided. Judd-Ofelt theory will be introduced to study the transition properties of $\mathrm{Nd}^{3+}$ ions. In Section 2.2, the energy transitions in neodymium system will be discussed. Various processes and parameters relevant to gain characterization, including lifetime, ETU, ASE, and rate equations, will be presented.

\subsection{Interaction between Light and Atomic Systems in Active Media}

\subsubsection{Einstein Coefficients}

An atom can be excited to a higher energy state when applying energy to the atom by means of electrical current or a photon. Only optical pumping will be considered in this thesis. In the Einstein treatment, a phenomenological description of the interaction of light with matter is given for the absorption and emission of radiation in a two level system, as illustrated in Fig. 2.1. It consists of an ensemble of isolated ions where only two levels are involved in the interaction. To begin with, the simplified two energy levels are considered unbroadened, and the radiation is described using photons.

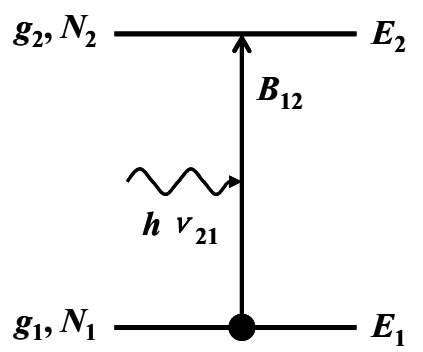

(a)

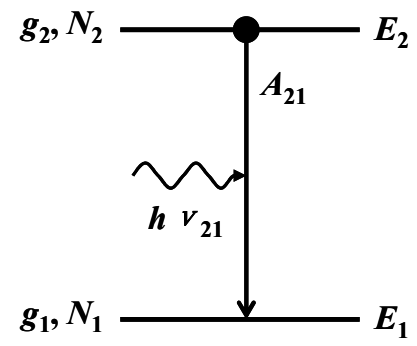

(b)

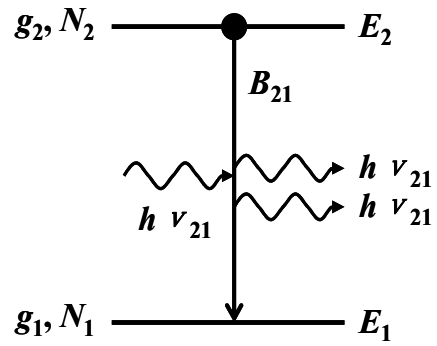

(c)

Fig. 2.1. Schematic representation of the (a) absorption, (b) spontaneous emission, and (c) stimulated emission process in a simplified two-level system.

Level 1 and 2 are the lower and upper level, respectively, with the corresponding energies $E_{1}$ and $E_{2}$ such that $E_{1}<E_{2}$. The number of atoms per unit 
volume in each of the two states is $N_{1}$ and $N_{2}$, respectively, and $g_{1}, g_{2}$ are the degeneracies of level 1 and 2, respectively. In thermal equilibrium, the distribution of population among the levels is described by Boltzmann statistics. The number of atoms per unit volume in the excited state 2 is given by the Boltzmann equation

$$
N_{2}=N_{1} \cdot \exp \left(-\left(E_{2}-E_{1}\right) / k_{B} T\right)
$$

where $k_{B}$ is Boltzmann's constant and $T$ is the absolute temperature [17].

When the system interacts with light, atoms in the lower state 1 can be promoted to the higher state 2 by absorbing a photon of energy equal to the energy difference between the two levels, as described in Fig. 2.1(a). Atoms found in the higher level 2 can relax spontaneously to level 1 either by emitting a photon of the same energy. This is the so-called spontaneous emission as depicted in Fig. 2.1(b). Spontaneous emission is a process governed by the Einstein $A$ coefficient, $A_{21}$. This represents the probability per unit time that the atom decays spontaneously from level 2 to level 1. Another process involved in the interaction between light and the atomic system is stimulated or induced emission, where a photon forces an atom in the excited state to decay by emitting another photon with the same phase as the incident photon (see Fig. 2.1(c)). This process, stimulated emission, contributes to the amplification of the light in an active medium. Stimulated absorption and emission are described by Einstein $B$ coefficients, $B_{12}$ and $B_{21}$, respectively.

In the case of interaction with light, or more generally with a radiation field, the population density of the ground state and first excited state is governed by absorption and emission transition rates, which are given by:

$$
\begin{aligned}
& \left.\frac{\partial N_{1}}{\partial t}\right|_{a b s}=-B_{12} \cdot \rho(v) \cdot N_{1}, \\
& \left.\frac{\partial N_{2}}{\partial t}\right|_{\text {stim }}=-B_{21} \cdot \rho(v) \cdot N_{2}, \\
& \left.\frac{\partial N_{2}}{\partial t}\right|_{\substack{\text { spont } \\
\text { em }}}=-A_{21} \cdot N_{2},
\end{aligned}
$$

where $\rho(v)$, the spectral energy density of the radiation per unit frequency, is assumed to be uniformly distributed near the transition frequency $v_{12} . \rho(v)$ is related to the intensity $I$ and the photon flux $\Phi$ by:

$$
\rho(v)=\frac{n}{c} \cdot I(v)=\frac{n h v}{c} \Phi(v)
$$


where $n$ is the real part of the refractive index of the medium, $c$ is the speed of light, and $h$ is Planck's constant. The absorption rate of photons with energy $h v$ is equal to the depopulation rate of the lower level. The Einstein absorption coefficient $B_{12}$ is related to the induced transition probability by $B_{12} \cdot \rho(v)$. The rate of photons generated at the radiative transition due to spontaneous emission is proportional to the population of the upper level. If the total number of atoms remains constant and the system is in thermal equilibrium a steady state situation arises where the transition rate from ground state to first excited state equals the transition rate from first excited state to ground state:

$$
B_{12} \cdot \rho(v) \cdot N_{1}=A_{21} \cdot N_{2}+B_{21} \cdot \rho(v) \cdot N_{2}
$$

Since the magnitude of the Einstein coefficients is solely an atomic property, they can be related to each other without loss of generality by considering the case of the atoms being in thermal equilibrium with a blackbody radiation field [17]. Using the Boltzmann distribution for describing the $N_{2} / N_{1}$ ratio in thermal equilibrium and Planck's law for the radiation density $I(v, T)$ of a blackbody

$$
I(v, T)=\frac{h v^{3}}{c_{0}{ }^{3}} \cdot \frac{1}{\exp \left(h v / k_{B} T\right)-1}
$$

the following relations between the Einstein coefficients can be derived [73]:

$$
\begin{aligned}
& g_{1} \cdot B_{12}=g_{2} \cdot B_{21}, \\
& A_{21}=\frac{8 \pi h v^{3} n^{3}}{c_{0}^{3}} B_{21} .
\end{aligned}
$$

It can be seen that the stimulated emission, the absorption, and the spontaneous emission are closely related to each other. In the steady-state case, knowing one of the Einstein coefficients, the others can be calculated and the two-level system is completely described.

\subsubsection{Absorption, Emission, and Gain}

As light traverses an amplifying medium of a finite length, a fraction of it may get either absorbed by the atoms of the medium, or may induce light emission by forcing atoms in the excited state to decay to a lower energy state. If the intensity of the beam at a certain location $\mathrm{z}$ in the medium is $I(z)$, the change in intensity per unit length for small signal amplification, satisfies a Beer's Law-like relation [74] 


$$
\frac{d I(z)}{d z}=\gamma(\lambda) I(z)
$$

where $\gamma(\lambda)$ is the gain coefficient $\left(\mathrm{cm}^{-1}\right)$, defined to contain both emission amplification and absorption losses. Also some atoms may decay by spontaneous emission, but since photons are emitted with equal probability in all directions, only the fraction emitted in the direction of the beam may contribute to the amplified spontaneous emission (ASE) which will be discussed in the later section. Under certain condition, ASE might contribute on the total intensity of light in the gain media and need to be subtracted when studying the small signal gain.

The photons added to the signal per unit time per unit volume can be written as

$$
\frac{d N_{2}}{d t}=\left[B_{21} g_{e m}(v) N_{2}-B_{12} g_{a b s}(v) N_{1}\right] \cdot \frac{I(z)}{c / n},
$$

where $g_{e m}(v)$ and $g_{a b s}(v)$ are the spectral line shape distributions of absorption and emission, respectively, which are used to describe the atom transitions. The change (increase for light amplification) in the energy density per unit time is

$$
\frac{d \rho}{d t}=\frac{d N}{d t} \cdot h v=\frac{d}{d t}\left(\frac{I(z)}{c / n}\right)
$$

With this, we can write the change in the beam intensity per unit length as

$$
\frac{d I(z)}{d z}=\left[B_{21} g_{e m}(v) N_{2}-B_{12} g_{a b s}(v) N_{1}\right] \cdot h v \cdot \frac{I(z)}{c / n}=\gamma(v) I(z)
$$

where the optical gain $\alpha(v)$ can be expressed in terms of the populations of the two manifolds as

$$
\gamma(v)=\left[B_{21} g_{e m}(v) N_{2}-B_{12} g_{a b s}(v) N_{1}\right] \cdot \frac{h v}{c / n} .
$$

We write the stimulated $\sigma_{e m}(v)$ emission and $\sigma_{a b s}(v)$ absorption cross sections of the transition as

$$
\sigma_{e m}(v)=B_{21} g_{e m}(v) \cdot \frac{h v}{c / n}
$$




$$
\sigma_{a b s}(v)=B_{12} g_{a b s}(v) \cdot \frac{h v}{c / n} .
$$

Therefore, the gain cross section $\sigma_{\text {gain }}(v)$ can be expressed in terms of the two cross sections as

$$
\sigma_{g a i n}(v)=\sigma_{e m}(v) N_{2}-\sigma_{a b s}(v) N_{1} .
$$

Amplification of light occurs when the term containing stimulated emission overcomes the one containing absorption, resulting in a positive net gain. The gain is determined by the two cross sections and the populations of the two levels. To design optical amplifiers and lasers, good knowledge of these parameters is required. Absolute values of the cross sections as well as spectral distributions are important.

The cross section is a fundamental property of the atom in a particular environment and represents a fictitious area within which the atom interacts (absorbs or emits) with an electromagnetic field incident on it.

In the wavelength $\lambda$ scale, the absorption cross section $\sigma_{a b s}(\lambda)$ in $\mathrm{cm}^{2}$ of rareearth ions can be calculated from the measured absorption spectrum by the equation:

$$
\sigma_{a b s}(\lambda)=\frac{\mathrm{a}_{a b s}(\lambda)}{N_{0}},
$$

where $\mathrm{a}_{a b s}$ is the absorption coefficient in $\mathrm{cm}^{-1}$, and $N_{0}$ is the ground-state population density in $\mathrm{cm}^{-3}$, which approximately equals the doping concentration of $\mathrm{Nd}^{3+}, N_{d}$, of the rare-earth ions in the host.

The emission cross section $\sigma_{e m}(\lambda)$ can be calculated by the measured emission cross section using Füchtbauer-Ladenburg theory, which will be discuss in the next section. Absorption and emission cross section can be derived from each other using the method of reciprocity, which will be discussed in Section 2.2.4.

\subsubsection{Füchtbauer-Ladenburg Theory}

Using the Einstein relation (Eq. 2.9) between the $B_{12}$ and $A_{21}$ coefficients, the emission cross section, as given by Eq. 2.14, can be related to the radiative lifetime $\tau_{\text {rad. }}$. This relationship is known as the Füchtbauer-Ladenburg equation [75]:

$$
\sigma_{e m}(v)=\frac{\lambda^{2}}{8 \pi n^{2} \tau_{r a d}} \cdot g(v)
$$

The radiative lifetime $\tau_{\text {rad }}$ can be calculated by Judd-Ofelt theory, which will be explained in Section 2.2.5. Although there are many possible line shapes, it is 
common to model the spectral line shape function as a Lorentzian distribution. After some simplifications, Eq. 2.19 can be translated to the form:

$$
\sigma_{e m}(\lambda)=\frac{\lambda^{4} B}{8 \pi c n^{2} \tau_{\text {rad }}} \frac{I(\lambda)}{\int I(\lambda) d \lambda}
$$

Where $B$ is the branching ratio of $\mathrm{Nd}^{3+}$ emission, and $I(\lambda)$ is the wavelength-dependent emission intensity. Using this equation, the emission cross section between an upper level and a lower level can be directly calculated by the measured emission spectrum.

\subsubsection{McCumber Theory}

An alternative way to obtain the emission or absorption cross section is to make use of the method of reciprocity. The McCumber theory [76] provides a treatment where a Boltzmann thermal population of the individual Stark levels is taken into account. Here the Stark splitting is assumed to be homogeneous and the relaxation within the Stark levels fast with respect to the manifold lifetime. The McCumber theory provides a relation between the emission cross section as a function of the absorption cross section as can be derived using Fig. 2.2 which schematically represents the Stark splitting, where $\sigma_{j i}(\lambda)$ and $\sigma_{i j}(\lambda)$ are the emission and absorption cross section of the transition, respectively, between two individual Stark levels $N_{1 i}$ and $N_{2 j}$ of the lower and upper manifold, respectively.

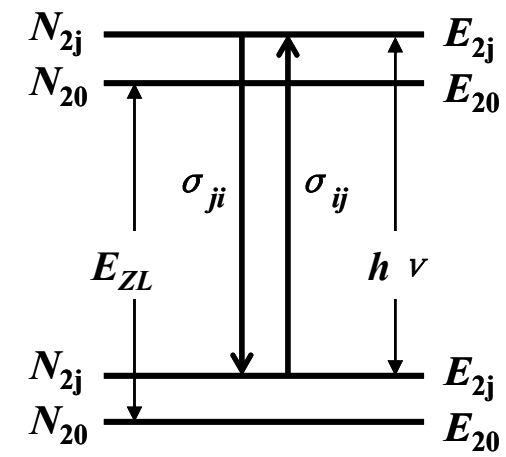

Fig. 2.2. Energy level scheme including Stark splitting.

By using the equality $\sigma_{j i}(\lambda)=\sigma_{i j}(\lambda)$, known as detailed balance or microscopic reciprocity, it can be derived that [77-79]:

$$
\sigma_{e m}(\lambda)=\frac{Z_{1}}{Z_{2}} \cdot \exp \left[h c\left(\frac{1}{\lambda_{Z L}}-\frac{1}{\lambda}\right) / k T\right] \cdot \sigma_{a b s}(\lambda)
$$

where the partition functions $Z_{1}$ and $Z_{2}$ of the Stark levels involved are given by [2]: 


$$
\begin{aligned}
& Z_{1}=\sum_{i} \exp \left[-\left(E_{1 i}-E_{10}\right) / k T\right], \text { and } \\
& Z_{2}=\sum_{j} \exp \left[-\left(E_{2 j}-E_{20}\right) / k T\right]
\end{aligned}
$$

The wavelength $\lambda_{\mathrm{ZL}}$ corresponds to the peak absorption wavelength and is related to the transition energy between the two lowest Stark levels, which is referred as zero-line energy:

$$
E_{Z L}=E_{20}-E_{10}=h c / \lambda_{Z L}
$$

If the Stark levels and the zero-line energy are known, the emission cross section may be determined from the measured absorption cross section, or vise versa.

\subsubsection{Judd-Ofelt Theory}

Knowledge of the transition intensities of $4 \mathrm{f}-4 \mathrm{f}$ transitions and of absorption and emission cross sections is the first step in investigating the performance of rare-earthion-doped laser and amplifiers. The Judd-Ofelt theory [80,81] is a successful model for the calculation and characterization of the optical transitions in rare-earth doped materials, which has been widely used in many previous investigations to predict the transition probabilities of rare-earth-ion doped polymer waveguides and study the feasibility of optical amplification and lasing [82-86]. It is based on the absorption spectrum of a rare-earth-ion-doped material.

The electric dipole line strength $S$ is adopted to describe the transition between two of the eigenstates of the ion in the Judd-Ofelt theory. The line strength $S$ between initial state $J$ characterized by $(S, L, J)$ and the final state $J^{\prime}$ given by $\left(S^{\prime}, L^{\prime}\right.$, $J$ ) can be written as [73-75]:

$$
S_{\text {calc }}\left(J \rightarrow J^{\prime}\right)=\sum_{t=2,4,6} \Omega_{t}\left|\left\langle(S, L) J\left\|U^{(t)}\right\|\left(S^{\prime}, L^{\prime}\right) J^{\prime}\right\rangle\right|^{2}=\Omega_{2} \cdot\left[U^{(2)}\right]^{2}+\Omega_{4} \cdot\left[U^{(4)}\right]^{2}+\Omega_{\mathrm{\sigma}} \cdot\left[U^{(6)}\right]^{2},
$$

where $\Omega_{t}(t=2,4,6)$ are the Judd-Ofelt parameters, and $U^{(t)}(t=2,4,6)$ are the doubly reduced matrix elements that depend only on angular momentum. As $U^{(t)}(t=2,4,6)$ are independent of the host, the values can be obtained from the literature $[82,87]$. The parameters $\Omega_{t}(t=2,4,6)$ are determined by measuring the line strength for a number of ground-state transition.

The measured line strengths $S_{\text {meas }}\left(J \rightarrow J^{\prime}\right)$ of the bands are determined using the following expression: 


$$
S_{\text {meas }}\left(J \rightarrow J^{\prime}\right)=\frac{3 \operatorname{ch}(2 J+1) n}{8 \pi^{3} \lambda e^{2} N_{0}} \cdot \frac{9}{\left(n^{2}+2\right)^{2}} \cdot \int \mathrm{a}(\lambda) d \lambda
$$

where $c$ is the velocity of light, $h$ is Planck's constant, $e$ is the elementary charge, $J$ is the angular momentum, $N_{0}$ is the density of ions, $\lambda$ is the mean wavelength of the absorption bands, $n$ is the wavelength-dependent refractive index which is determined from Sellmeier's dispersion equation, and $\int \mathrm{a}(\lambda) \mathrm{d} \lambda$ is the integrated absorption coefficient from measured absorption bands of rare-earth ions.

By a least-squares fit of $S_{\text {meas }}$ to $S_{\text {calc }}$, the values of the three parameters $\Omega_{t}(\mathrm{t}$ $=2,4,6)$ are obtained from Eq. 2.24 and 2.25. The line strengths corresponding to the transitions from the upper manifold states to their corresponding lower-lying manifold states are then calculated by Eq. 2.24, based on which the radiative decay rates $A_{J J}$, radiative lifetimes $\tau_{\text {rad }}$, and fluorescence branching ratios $B$ can be calculated as follows:

$$
\begin{aligned}
& A\left(J \rightarrow J^{\prime}\right)=\frac{64 \pi^{4} e^{2}}{3 h(2 J+1) \lambda^{3}} \frac{n\left(n^{2}+2\right)^{2}}{9} S_{\text {calc }}\left(J \rightarrow J^{\prime}\right), \\
& \tau_{\text {rad }}=\frac{1}{\sum A\left(J \rightarrow J^{\prime}\right)} \tau_{\text {rad }}, \\
& B\left(J \rightarrow J^{\prime}\right)=\frac{A\left(J \rightarrow J^{\prime}\right)}{\sum A\left(J \rightarrow J^{\prime}\right)}=A\left(J \rightarrow J^{\prime}\right) \tau_{\text {rad }} .
\end{aligned}
$$

In Chapter 3, the Judd-Ofelt theory will be applied to study $\mathrm{Nd}^{3+}$-doped waveguides.

\subsection{Amplification and Lasing in $\mathrm{Nd}^{3+}$}

\subsubsection{Energy Transitions of $\mathrm{Nd}^{3+}$ Ions}

The rare earths of interest are the lanthanides, which are chemical elements characterized by filling of the $4 f$ shell. They are placed in the sixth period of the periodic table with the atomic number varying from 58 (cerium) to 71 (lutetium), The $4 f$ electrons in lanthanides have almost no contribution to the chemical valence, thus the atom can easily lose the $6 \mathrm{~s}$ electrons and also one electron from the $4 f$ shell, so they are most stable as trivalent ions.

Neodymium is part of the lanthanide series in the periodic table of the elements and has the following electron configuration:

$$
1 s^{2} 2 s^{2} p^{6} 3 s^{2} p^{6} d^{10} 4 s^{2} p^{6} d^{10} f^{4} 5 s^{2} p^{6} 6 s^{2}
$$

Ionized in the $\mathrm{Nd}^{3+}$ state, both the $6 s^{2}$ electrons and one $4 f$ electron are 
missing. The electron configuration leading to this ionized state is:

$$
1 s^{2} 2 s^{2} p^{6} 3 s^{2} p^{6} d^{10} 4 s^{2} p^{6} d^{10} f^{3} 5 s^{2} p^{6}
$$

All shells, except the $4 f$-shell, are filled and thus give no contribution to the total orbital and spin momentum . Luminescent transitions within the $4 f$-shell can occur due to mixing with allowed transitions. Such metastable states in which the electrons reside, give rise to long luminescent lifetimes and low absorption coefficients.

Figure 2.3 shows a schematic representation of a selection of possible energy transitions of neodymium ions [88, 89]. According to Hund's rules, the ground state represents the electron state which has the highest total spin $S$, the lowest angular momentum $L$, and the lowest total angular momentum $J$, and is the ${ }^{4} \mathrm{I}_{9 / 2}$ state in the neodymium system.

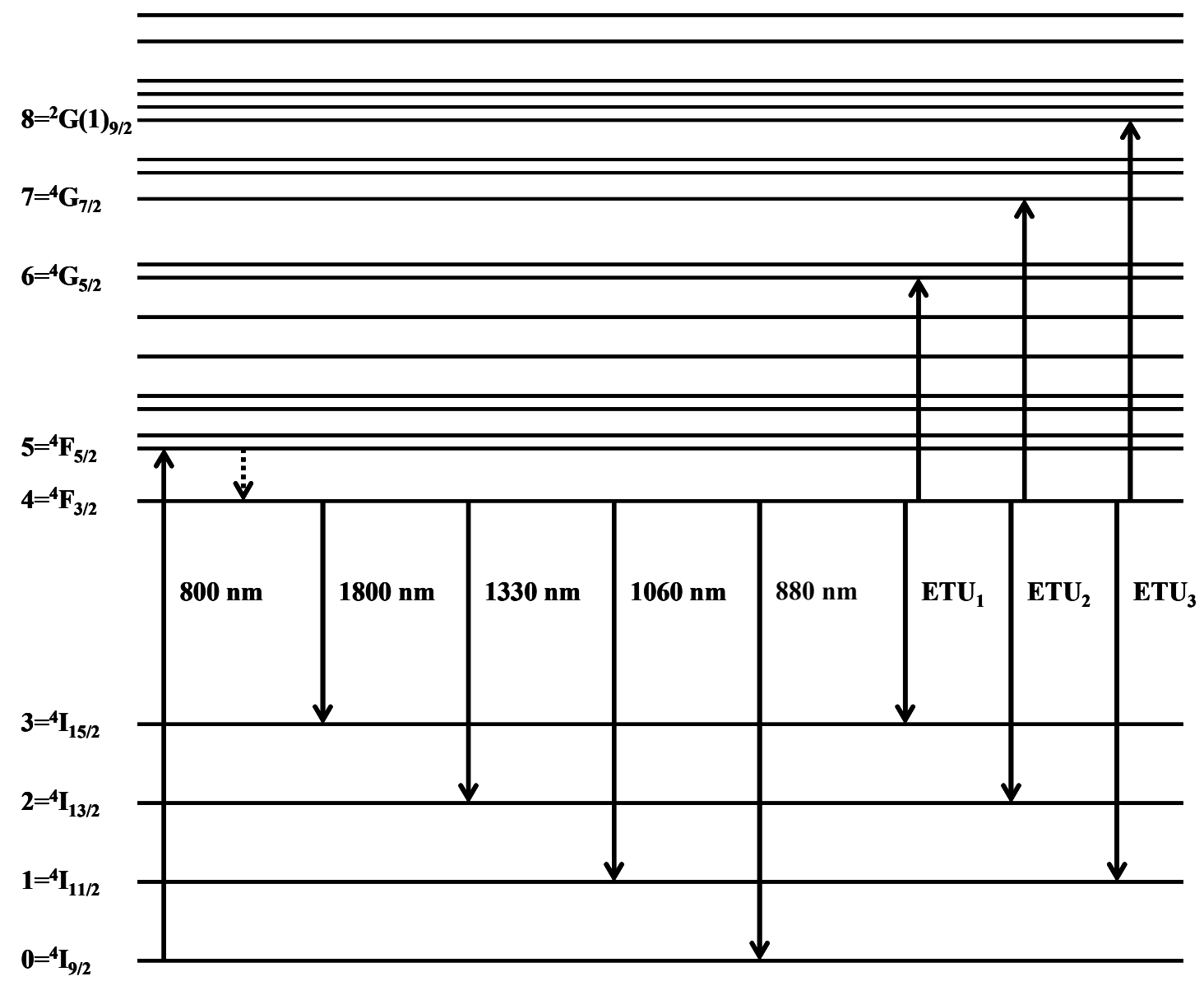

Fig. 2.3. A schematic of the energy levels of $\mathrm{Nd}^{3+}$ ions.

In Fig. 2.3, the absorption of $800 \mathrm{~nm}$ pump photons causes excitation of the $\mathrm{Nd}^{3+}$ to the ${ }^{4} \mathrm{~F}_{5 / 2}$ level. After a fast relaxation, the ion remains in the metastable ${ }^{4} \mathrm{~F}_{3 / 2}$ level with a lifetime that is typically in the order of hundreds of microseconds in case of low concentrations in different hosts [47, 90-94]. Similar absorption processes take place around, for instance, $580 \mathrm{~nm}$ and $745 \mathrm{~nm}$. The corresponding pump levels $\left({ }^{4} \mathrm{~F}_{5 / 2}\right.$, 
${ }^{4} \mathrm{~F}_{7 / 2},{ }^{4} \mathrm{G}_{5 / 2}$, and ${ }^{4} \mathrm{G}_{7 / 2}$ ) as well as the lower laser levels are depleted by fast multiphonon relaxation leading to significant excitation solely in the ${ }^{4} \mathrm{~F}_{3 / 2}$ level [88, 89]. After relaxation to the ${ }^{4} \mathrm{~F}_{3 / 2}$ level, several radiative decays to the ${ }^{4} \mathrm{I}_{9 / 2},{ }^{4} \mathrm{I}_{11 / 2},{ }^{4} \mathrm{I}_{13 / 2}$, and ${ }^{4} \mathrm{I}_{15 / 2}$ levels, corresponding to the wavelength bands centered at $880 \mathrm{~nm}, 1060 \mathrm{~nm}$, $1330 \mathrm{~nm}$ and $1800 \mathrm{~nm}$, respectively, can take place.

The gain coefficient, as derived from the two-level case in Section 2.2.1, is also vaild in multi-level systems and proportional to the population of the excited state. A deterioration arises if other competing depopulation mechanisms are present. Other mechanisms may occur, e.g. if a multilevel system is considered, or interactions with the host material and other ions are taken into account.

\subsubsection{Lifetime}

The lifetime of a given energy level is the time constant describing the exponential decay of ions from that level. It is inversely proportional to the probability per unit time of the decay of an ion from that level. The number of ions excited to a given level is proportional to the intensity of spontaneous emission from that level. The emitted intensity can be plotted as a function of time to obtain a luminescence decay curve. In the absence of other energy-transfer processes, the luminescence decay is represented by a single exponential curve. The inverse of the luminescent lifetime can be written as a sum of inverse lifetimes which represent the different decay paths. These can be divided into radiative and non-radiative decay. Radiative decay results in the spontaneous emission of a photon, while in non-radiative decay the energy is transferred to phonons, or vibrations of the host material. The following equation relates the various contributions to the luminescence lifetime:

$$
\frac{1}{\tau_{\text {lum }}}=\frac{1}{\tau_{\text {rad }}}+\frac{1}{\tau_{\text {non-rad }}},
$$

where $\tau_{l u m}$ is the luminescence lifetime, $\tau_{\text {rad }}$ is the radiative lifetime, and $\tau_{\text {non-rad }}$ is the non-radiative lifetime. Non-radiative decay occurs by energy transfer to the host material through a number of phonons with total energy equal to the energy gap between the levels, called multi-phonon relaxation.

\subsubsection{Energy-transfer Upconversion}

Besides the transitions between energy levels due to absorption, stimulated emission, and radiative and non-radiative decay, transitions caused by energy transfer between neighboring ions play an important role. A process involving energy transfer between ions is illustrated in Fig. 2.4, where two excited ions interact, with energy from the donor ion being transferred to the acceptor, promoting it to a higher energy state. This process is called energy-transfer upconversion (ETU). 


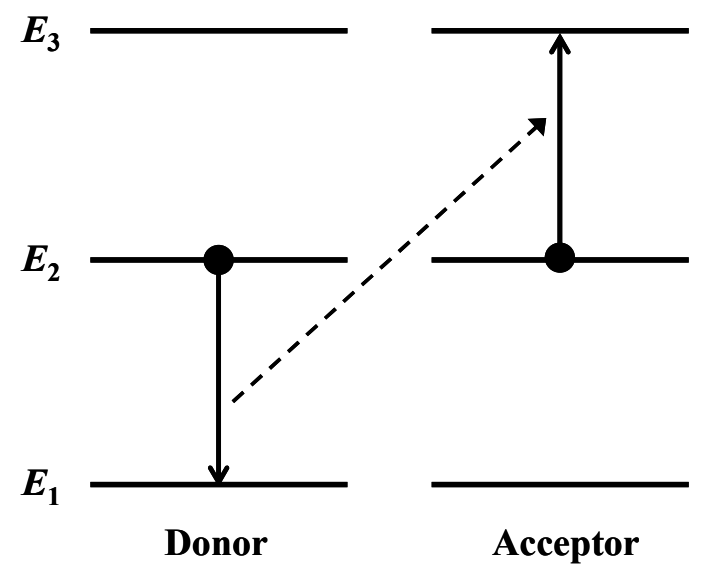

Fig. 2.4. $\mathrm{Nd}^{3+}-\mathrm{Nd}^{3+}$ inter-ionic energy-transfer upconversion (ETU).

In $\mathrm{Nd}^{3+}$-doped materials, the three ETU processes originating in the metastable ${ }^{4} \mathrm{~F}_{3 / 2}$ level (see Fig. 2.3) have a significant impact on the performance of amplifiers and lasers. To study the effect of ETU, three ETU processes are taken into account. Since all terminating levels of ETU processes in the $\mathrm{Nd}^{3+}$ system exhibit fast multiphonon decays into the ${ }^{4} \mathrm{~F}_{3 / 2}$ and ${ }^{4} \mathrm{I}_{9 / 2}$ levels, they lead to similar results concerning the population dynamics $[88,89]$. Therefore, the three ETU processes can be expressed by a single macroscopic parameter $W_{\mathrm{ETU}}$. The ETU parameters can be determined from either the non-exponential luminescent decay of the first excited state, or the gain measurement and corresponding simulation. More details of the relevant studies will be discussed in the following Sections and Chapters.

\subsubsection{Amplified Spontaneous Emission}

Stimulated transitions due to previously generated spontaneous emission also lead to depopulation of the metastable state. This mechanism is known as amplified spontaneous emission (ASE) [95, 96].

The ASE intensity is, like the pump and signal intensity, carried by a guided mode in the amplifier. It is an indication of optical gain in the rare-earth-ion systems. The influence of ASE can be calculated by spectrally resolving the evolution of the ASE spectrum both in the forward and backward directions along the amplifier.

It is necessary to remove the influence of ASE when measuring optical net gain. Besides, the ASE at the output of the amplifier gives rise to noise [97].

\subsubsection{Rate Equations}

To simulate the local population of the excited state at a point along the amplifier as shown in the energy level diagram (Fig. 2.3), the rate equations of $\mathrm{Nd}^{3+}$ were derived as follows $[88,89]$ :

$$
\frac{d N_{8}}{d t}=W_{3} N_{4}^{2}-\frac{N_{8}}{\tau_{8}},
$$




$$
\begin{aligned}
& \frac{d N_{7}}{d t}=W_{2} N_{4}^{2}+\frac{N_{8}}{\tau_{8}}-\frac{N_{7}}{\tau_{7}}, \\
& \frac{d N_{6}}{d t}=W_{1} N_{4}^{2}+\frac{N_{7}}{\tau_{7}}-\frac{N_{6}}{\tau_{6}}, \\
& \frac{d N_{5}}{d t}=R_{05}+\frac{N_{6}}{\tau_{6}}-\frac{N_{5}}{\tau_{5}}, \\
& \frac{d N_{4}}{d t}=\frac{N_{5}}{\tau_{5}}-\frac{N_{4}}{\tau_{4}}-2\left(W_{1}+W_{2}+W_{3}\right) N_{4}^{2}, \\
& \frac{d N_{3}}{d t}=\beta_{43} \frac{N_{4}}{\tau_{4}}-\frac{N_{3}}{\tau_{3}}+W_{1} N_{4}^{2}, \\
& \frac{d N_{2}}{d t}=\beta_{42} \frac{N_{4}}{\tau_{4}}+\frac{N_{3}}{\tau_{3}}-\frac{N_{2}}{\tau_{2}}+W_{2} N_{2}^{2}, \\
& \frac{d N_{1}}{d t}=\beta_{41} \frac{N_{4}}{\tau_{4}}+\frac{N_{2}}{\tau_{2}}-\frac{N_{1}}{\tau_{1}}+W_{3} N_{4}^{2}, \\
& \frac{d N_{0}}{d t}=-R_{05}+\beta_{40} \frac{N_{4}}{\tau_{4}}+\frac{N_{1}}{\tau_{1}},
\end{aligned}
$$

where $N_{i}$ and $\tau_{i}(i=0-8)$ are the population density and lifetime of each level, respectively, $\beta_{4 \mathrm{i}}(i=0-3)$ are the branching ratios of transitions from ${ }^{4} \mathrm{~F}_{3 / 2}, W_{1}, W_{2}$, and $W_{3}$ are the ETU parameters, and $R_{05}$ is the rates of pump absorption.

For simulating optical gain in $\mathrm{Nd}^{3+}$-doped waveguides at $880 \mathrm{~nm}, 1064 \mathrm{~nm}$, and $1330 \mathrm{~nm}$, the rate equations can be further simplified.

The $\mathrm{Nd}^{3+}$ transition ${ }^{4} \mathrm{~F}_{3 / 2} \rightarrow{ }^{4} \mathrm{I}_{9 / 2}$ around $880 \mathrm{~nm}$ is a three-level transition. $\mathrm{Nd}^{3+}$ ions are excited around $800 \mathrm{~nm}$ from the ground state ${ }^{4} \mathrm{I}_{9 / 2}$ to the pump level ${ }^{4} \mathrm{~F}_{5 / 2}$ followed by a fast decay to the metastable excited state ${ }^{4} \mathrm{~F}_{3 / 2}$. Since all other excited states in $\mathrm{Nd}^{3+}$ exhibit fast multi-phonon quenching and have very short lifetimes, the rate equations describing the population mechanisms of this system can be simplified as follows:

$$
\begin{aligned}
& d N_{4} / d t=R_{05}-R_{40}+R_{04}-\tau_{4}^{-1} N_{4}-W_{E T U} N_{4}^{2}, \\
& N_{0}=N_{d}-N_{4},
\end{aligned}
$$

where $N_{4}$ and $\tau_{4}$ are the population density and lifetime of the ${ }^{4} \mathrm{~F}_{3 / 2}$ level, respectively, $N_{0}$ is the ground-state population density, and $N_{d}$ is the dopant concentration. The three ETU processes originating in the metastable ${ }^{4} \mathrm{~F}_{3 / 2}$ level of the $\mathrm{Nd}^{3+}$ system were taken into account and expressed by a single macroscopic parameter $W_{\text {ETU }}$ in the simulation, where it is assumed that the upconverted ion returns instantaneously to the 
metastable state by fast multiphonon relaxation. The rates of pump absorption $R_{05}$, signal re-absorption $R_{04}$, and stimulated emission $R_{40}$ can be expressed as follows:

$$
\begin{aligned}
& R_{05} \approx \sigma^{p}{ }_{a b s} \frac{\lambda_{p}}{h c} I_{p} N_{0}, \\
& R_{04} \approx \sigma^{s}{ }_{a b s} \frac{\lambda_{s}}{h c} I_{s} N_{0}, \\
& R_{40} \approx \sigma^{s}{ }_{e m} \frac{\lambda_{s}}{h c} I_{s} N_{4},
\end{aligned}
$$

where $\sigma_{a b s}^{p}, \sigma_{a b s}^{s}$, and $\sigma_{e m}^{s}$ are the pump-absorption, signal-absorption, and stimulatedemission cross sections, $\lambda_{p}$ and $\lambda_{s}$ are the wavelengths and $I_{p}$ and $I_{s}$ the intensities of pump and signal light, respectively, launched into the waveguide in propagation direction $z$, and $h$ is Planck's constant. At steady state, we can solve the above equations analytically.

Since the terminating states ${ }^{4} \mathrm{I}_{11 / 2}$ and ${ }^{4} \mathrm{I}_{13 / 2}$ of the transitions at $1064 \mathrm{~nm}$ and $1330 \mathrm{~nm}$, respectively, exhibit a very short lifetime on the order of a few ns, these transitions constitute four-level systems. The re-absorption at the signal wavelength can be neglected, and the rate equations can be simplified further as follows:

$$
\begin{aligned}
& d N_{4} / d t=R_{05}-R_{4 i}-\tau_{4}^{-1} N_{4}-W_{E T U} N_{4}^{2} \quad i=1,2, \\
& N_{0}=N_{d}-N_{4}, \\
& R_{4 i} \approx \sigma_{c m}^{s} \frac{\lambda_{s}}{h c} I_{s} N_{4} \quad i=1,2,
\end{aligned}
$$

In addition to discretization in the propagation direction $z$, a radial discretization $[88,89]$ was included in the simulation. The percentage of pump and signal power inside the active region, which contributes to the population dynamics, is taken into account in the simulation. The optical mode profiles were then approximated by Gaussian profiles. The amount of pump or signal power $P_{P / S}(r, z)$ passing through a circle of radius $r$ at a propagation distance $z$ is described by the equation

$$
P_{(P / S)}(r, z)=P_{P / S, \text { total }}(z)\left[1-\exp \left(\frac{-2 r^{2}}{w_{P / S}^{2}}\right)\right] \text {, }
$$

where $P_{P / S, \text { total }}(z)$ is the total power propagating at distance $z$ and $w_{P / S}$ the Gaussian beam waist of pump and signal mode, respectively, which is defined as the radial distance at which the optical intensity drops to $1 / \mathrm{e}^{2}$ of its peak value. The total remaining power of pump and signal beams were each re-distributed in a Gaussian 
profile before entering the next longitudinal propagation step.

Gain investigation in $\mathrm{Nd}^{3+}$ systems will be presented in Chapter 4 .

\subsection{Summary}

The theoretical background of interaction between light and atomic system in active media relevant for this thesis has been presented. The properties of the optically active rare-earth dopants, especially neodymium, have been presented with a focus on optical gain. The main processes and parameters for optical amplifiers and lasers, absorption and stimulated emission, their cross sections, spotaneous emission and its lifetime, ETU, and ASE have been discussed and will be used for spectroscopic characterization and gain investigation. Rate equations of $\mathrm{Nd}^{3+}$ have been explained and simplified for the simulation of gain in a later Chapter. 
Chapter 2 


\section{Chapter 3}

\section{$\mathrm{Nd}^{3+}$-doped Waveguides: Fabrication and Optical Characterization}

In this Chapter the fabrication and optical characterization of Nd-doped waveguides are discussed. $\mathrm{Nd}^{3+}$-complex-doped polymer films were deposited by spin-coating, and channel waveguides were realized by a simple and reproducible procedure mainly based on spin-coating and photo-definition. $\mathrm{Al}_{2} \mathrm{O}_{3}: \mathrm{Nd}^{3+}$ films were deposited on thermally oxidized silicon wafers using reactive co-sputtering, and channel waveguides were fabricated using reactive ion etch (RIE) techniques. Optical losses in the two types of $\mathrm{Nd}^{3+}$ doped waveguides were investigated and compared. Both the $\mathrm{Nd}^{3+}$-complexdoped polymer waveguides and $\mathrm{Al}_{2} \mathrm{O}_{3}: \mathrm{Nd}^{3+}$ waveguide have very low losses at the visible and near infrared wavelength, and $\mathrm{Al}_{2} \mathrm{O}_{3}: \mathrm{Nd}^{3+}$ waveguides show a broadband transmission window extended to telecommunication wavelength range. The absorption bands of $\mathrm{Nd}^{3+}$ were observed in the visible and near infrared range, based on which the Judd-Ofelt analysis, was applied to study the transition properties of $\mathrm{Nd}^{3+}$ ions in the two hosts. Photoluminescence spectra of $\mathrm{Nd}^{3+}$ were experimentally studied, and three luminescence peaks near $880 \mathrm{~nm}, 1060 \mathrm{~nm}$, and $1330 \mathrm{~nm}$, corresponding to the transitions ${ }^{4} F_{3 / 2} \rightarrow$ ${ }^{4} I_{9 / 2},{ }^{4} F_{3 / 2} \rightarrow{ }^{4} I_{11 / 2}$, and ${ }^{4} F_{3 / 2} \rightarrow{ }^{4} I_{13 / 2}$, respectively, were observed. The luminescence lifetime of $\mathrm{Nd}^{3+}$ ions in the two hosts was measured, and ETU parameters of $\mathrm{Nd}^{3+}$ in $\mathrm{Al}_{2} \mathrm{O}_{3}$ were derived from the luminescence decay curves. The characterization results indicate that these two $\mathrm{Nd}^{3+}$-doped materials are well suited for optical amplification and lasing. 


\subsection{Introduction}

Neodymium-doped waveguide lasers [27-29] and amplifiers [28, 29, 31-33] are of interest for applications at the ion's specific emission wavelengths and have been widely studied. The trivalent neodymium ion $\left(\mathrm{Nd}^{3+}\right)$ is arguably the most important active rare-earth ion for the demonstration of significant gain and laser operation due to the large emission cross-section of its four-level transition $\left({ }^{4} \mathrm{~F}_{3 / 2} \rightarrow{ }^{4} \mathrm{I}_{11 / 2}\right)$ at approximately $1060 \mathrm{~nm}$, resulting in a very low laser threshold. On the other hand, emissions on the ${ }^{4} \mathrm{~F}_{3 / 2} \rightarrow{ }^{4} \mathrm{I}_{9 / 2}$ ground-state transition at $880 \mathrm{~nm}$ and ${ }^{4} \mathrm{~F}_{3 / 2} \rightarrow{ }^{4} \mathrm{I}_{9 / 2}$ excited-state transition at $1330 \mathrm{~nm}$ are interesting for signal amplification in integrated optical applications, e.g. data transmission in optical interconnects [1-4] and medical diagnostics $[13,14]$.

In this chapter, two types of $\mathrm{Nd}^{3+}$-doped waveguides, $\mathrm{Nd}^{3+}$-complex-doped polymer and $\mathrm{Al}_{2} \mathrm{O}_{3}: \mathrm{Nd}^{3+}$ waveguides are discussed. They have been fabricated based on different fabrication techniques. The optical propagation loss of waveguides in a broad wavelength range has been experimentally investigated. By subtracting the background loss of waveguides, the absorption of $\mathrm{Nd}^{3+}$ in the two hosts has been characterized. The photoluminescence spectra of the $\mathrm{Nd}^{3+}$ at three transitions ${ }^{4} \mathrm{~F}_{3 / 2} \rightarrow$ ${ }^{4} \mathrm{I}_{9 / 2},{ }^{4} \mathrm{~F}_{3 / 2} \rightarrow{ }^{4} \mathrm{I}_{11 / 2}$, and ${ }^{4} \mathrm{~F}_{3 / 2} \rightarrow{ }^{4} \mathrm{I}_{13 / 2}$, around $880 \mathrm{~nm}, 1060 \mathrm{~nm}$, and $1330 \mathrm{~nm}$, respectively, have been observed, and the luminescence lifetime of $\mathrm{Nd}^{3+}$ in the two host materials has been experimentally determined. Key spectroscopic parameters have been determined based on experimental measurements and the feasibility of optical amplification and lasing in these two $\mathrm{Nd}^{3+}$-doped systems is discussed.

\section{2 $\mathrm{Nd}^{3+}$-complex-doped Polymer Waveguide Fabrication}

Polymer waveguides are promising candidates for integrated optical devices due to their high packaging density, low cost, compatibility with other materials, low optical loss in the near-infrared wavelength range, and ease of fabrication and modification of their chemical structure. This latter property offers enormous flexibility in the design of optical waveguide amplifier and laser media. Rare-earth doped polymer waveguides used for optical amplification and lasing have been investigated in the past. However, polymer-based $\mathrm{Nd}^{3+}$-doped channel waveguides that exploit the potential simplicity of polymer deposition and micro-structuring have as yet not been demonstrated and the application of polymers in integrated active devices is limited by the long-time stability at the high excitation power.

Polymer systems based on low-cost and commonly used (in adhesives, coatings, encapsulants) epoxy prepolymers were applied in this work to formulate the optical gain medium of the channel waveguides. Besides, a photodefinable epoxy was chosen as the cladding material, because it can be patterned with simple processes, including spin-coating, photo-lithography, and thermal curing.

\subsubsection{Film Deposition}

Incorporation of rare-earth ions into polymers is challenging due to the immiscibility of their salt precursors with organic solvents. This problem can be overcome by encapsulating the ions with organic ligands to form stable complexes that can be easily dispersed in polymer solutions. Furthermore, the ligands can serve to shield the dopant ion from impurities in the surrounding matrix that otherwise would quench the luminescence [30]. In addition to facilitating doping, organic complexes may also 
incorporate antenna chromophores, which act as sensitizers for the ion by intramolecular energy transfer, thereby enhancing the efficiency of light absorption $[98,99]$. An alternative route for achieving doping is by employing silica colloidal spheres as rare-earth precursors. Provided that there is a match between the indices of the polymer and the colloids, the produced nanocomposites show high gain and a long luminescence lifetime, thereby easing the requirement for high pump powers [100]. Various Nd complexes have been synthesized, of which the luminescence properties have been investigated with a view to developing optical amplifiers [98, 101-105].

Another problem may arise in these structures due to the overtone vibrations of $\mathrm{C}-\mathrm{H}$ and $\mathrm{O}-\mathrm{H}$ bonds in the complex and the polymer (in its organic solvent), respectively, which are known to quench the luminescence by coupling of the excited state of the ion to the vibrational modes of these bonds $[49,106]$. This effect can be mitigated by reducing the energy of the fundamental vibration through substitution of the hydrogen atoms by lower reduced mass atoms such as deuterium, fluorine, or chlorine. In order to suppress the luminescence quenching of $\mathrm{Nd}^{3+}$ dopant ions, fluorinated chelates replaced part of the $\mathrm{C}-\mathrm{H}$ bonds by $\mathrm{C}-\mathrm{F}$ bonds in the complex used, while the neutral ligands eliminated the $\mathrm{H}_{2} \mathrm{O}$ molecules.

In this work, in order to overcome the insolubility problem of the inorganic precursor salts of the $\mathrm{Nd}^{3+}$ dopant ions in the polymer host and suppress the luminescence quenching of rare-earth dopants, the ions were encapsulated in a stable organic complex. $\mathrm{Nd}(\mathrm{TTA})_{3}$ phen $(\mathrm{TTA}=$ thenoyltrifluoroacetone, phen $=1,10$ phenanthroline), depicted in Fig. 3.1. Nd(TTA) $)_{3}$ phen $(1 \mathrm{mMol})$ was synthesized by following Ref [107]. TTA is a fluorinated ligand, while phen is natural. $1 \mathrm{mmol}$ $\mathrm{Nd}\left(\mathrm{NO}_{3}\right)_{3} \cdot 6 \mathrm{H}_{2} \mathrm{O}$ was dissolved in $20 \mathrm{ml}$ of methanol. TTAH $(3 \mathrm{mMol})$ was dissolved in $15 \mathrm{ml}$ of methanol in another glass beaker. The $\mathrm{Nd}\left(\mathrm{NO}_{3}\right)_{3}$ solution was poured into the TTAH solution and stirred for 30 minutes. A saturated solution of $\mathrm{NaOH}$ in methanol (with $\sim 5 \%$ water) was added gradually with stirring to $\mathrm{pH} 8$. The solution was stirred for $0.5 \mathrm{~h}$ after addition of $\mathrm{NaOH}$ and a clear solution was obtained. After addition of $1 \mathrm{mmol}$ of phen to the $\mathrm{Nd}$ solution, a white powder of $\mathrm{Nd}(\mathrm{TTA})_{3}$ phen was precipitated from the solution. After stirring for an additional $0.5 \mathrm{~h}$, the white product was collected by filtration under suction. The $\mathrm{Nd}(\mathrm{TTA})_{3}$ phen in the power form obtained in this way was washed with methanol and dried in a vacuum oven.

These organic ligands give a uniform size of the rare-earth-ion complex, which is typically below $10 \mathrm{~nm}[108,109]$. The small size of the rare-earth-ion complex as well as its transparency over the wavelength range of interest, make effects of the prolate geometry on the photophysical properties negligible. Compared to nanoparticle-doped polymers [110], polymers doped with an organic complex offer the advantages of a homogeneous distribution of active ions, allowing for higher doping concentrations before luminescence quenching occurs and lower sidewall roughness of waveguide channels.

The $\mathrm{Nd}^{3+}$-complex, $\mathrm{Nd}(\mathrm{TTA})_{3}$ phen, was dissolved into a solution of 6FDA/UVR that is based on 4,4'-(Hexafluoroisopropylidene)diphthalic anhydride (6FDA) and 3,4-epoxycyclohexylmethyl-3,4-epoxycyclohexane carboxylate (UVR), which is a fluorinated polymer material with low loss in the near infrared wavelength range. 


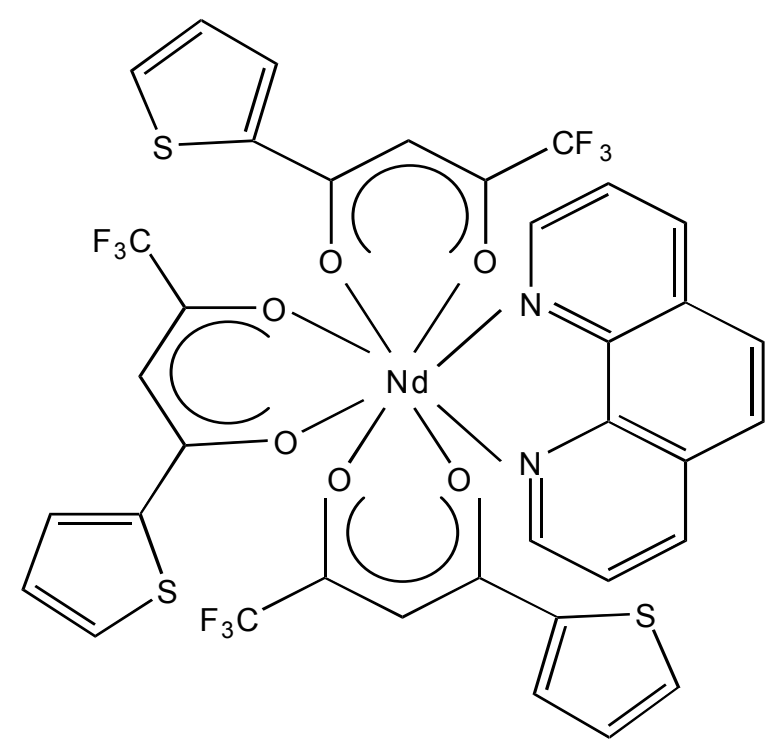

Fig. 3.1. The chemical structure of $\mathrm{Nd}(\mathrm{TTA})_{3}$ phen.

6-FDA (Aldrich) is a highly fluorinated molecule which further decreases the luminescence quenching originating from high-energy vibrations of $\mathrm{C}-\mathrm{H}$ and $\mathrm{O}-\mathrm{H}$ bonds in the polymer host. It was combined with the fluid epoxy monomer, UVR (Aldrich), whose molecules contain only aliphatic rings equipped with two highly reactive epoxy groups to yield a low refractive index to match the low-refractiveindex cladding material after cross-linking with the 6-FDA. The chemical structure of the 6-FDA/UVR network is presented in Fig. 3.2. To achieve a spin-coated film with good optical quality, a high boiling point solvent, Gamma-butyrolactone (Aldrich), was used, which due to its polar nature is capable of dissolving the fluorinated material.

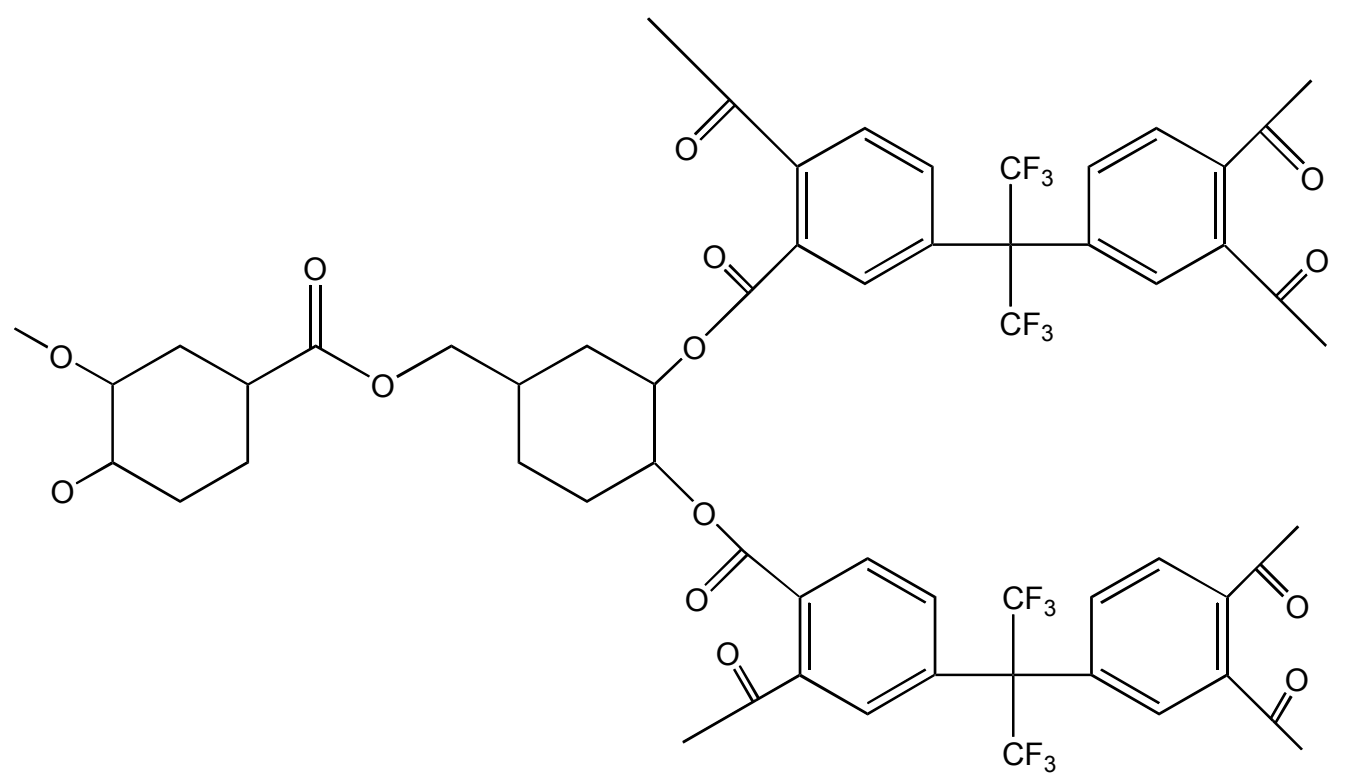

Fig. 3.2. The chemical structure of the 6-FDA/UVR network. 
The 6-FDA/UVR solution was synthesized as follows. $0.5 \mathrm{~g}$ FDA (Aldrich) was dissolved completely in $1.5 \mathrm{~g}$ Gamma-butyrolactone (Aldrich) and $1 \mathrm{~g}$ cyclopentanone (Aldrich) in a water bath for $1.5 \mathrm{~h}$. Nd(TTA) $)_{3}$ phen was dissolved in the FDA solution and $0.5 \mathrm{~g}$ UVR (Aldrich) was added thereafter.

Films of $\mathrm{Nd}(\mathrm{TTA})_{3}$ phen-doped 6-FDA/epoxy were achieved by depositing on thermally oxidized wafers using spin-coating and post-baking. The refractive index and the thickness as determined with a prism coupler were 1.53 (at $633 \mathrm{~nm}$ ) and 4.3 $\mu \mathrm{m}$, respectively. Figure 3.3 shows an atomic force microscope (AFM) image of the spin-coated $\mathrm{Nd}$ (TTA) 3 phen-doped 6-FDA/UVR film. The root mean square (rms) roughness of the film surface is $\sim 1.1 \mathrm{~nm}$.

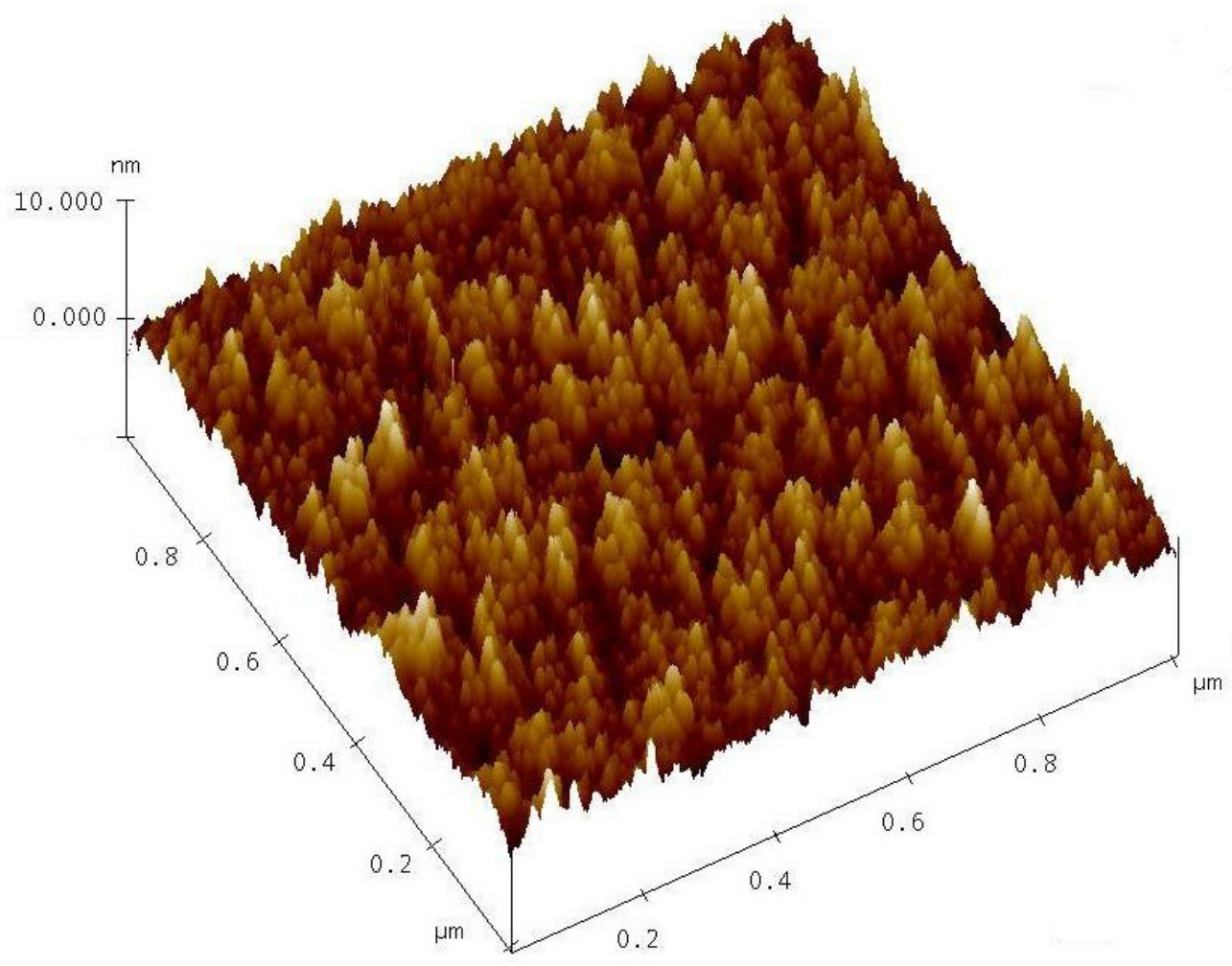

Fig. 3.3. AFM image of a $\mathrm{Nd}(\mathrm{TTA})_{3}$ phen-doped 6-FDA/epoxy film surface.

\subsubsection{Waveguide Structuring}

The functionalities of active doping and photo-definition were divided over two different polymers. While the properties of the core material were optimized to incorporate the $\mathrm{Nd}^{3+}$-complex, the cladding material was chosen for its simple processing properties, especially the straight-forward photodefinition of channel waveguide structures. An important requirement for a photodefinable epoxy polymer waveguide material is the solid nature of the epoxy prepolymer, which then prevents the fading by diffusion of the latent image of the photoacid catalyst in the epoxy matrix after masked UV exposure. Moreover, a solid material layer is required for contact mask alignment. 
In order to reduce the yellowing after aging owing to the high content of aromatic epoxies, which occurs in other photodefinable polymers such as the diglycidylether of bisphenol A (DGEBA) formulated in previous work [52], a cycloaliphatic epoxy prepolymer formulation (CHEP) [111] was identified as a suitable material, since it contains only saturated cyclohexyl groups with terminal epoxy groups. CHEP acts as a negative photoresist and fulfils the aforementioned attractive processing requirements, while it is also characterized by low optical loss and low yellowing properties. The main ingredient is EHPE-3150 of Daicel, which is the condensation product of 1-2-Epoxy-4(2-oxiranyl)-Cyclohexane of the 2,2bis(Hydoroxy methyl)1-butanol / (3'-4'-Epoxycyclohexane)methyl 3'-4'Epoxycyclohexyl-carboxylate mixture, sketched in Fig. 3.4. A photoacid generator (PAG) based on Triarylsulfonium hexafluorophosphate (UVI 6992 of Union Carbide) was used as photoinitiator. This PAG is known to induce less yellowing compared to the more commonly used PAG based on Triarylsulfonium hexafluoroantimonate. The photoacid-catalyzed epoxy was cross-linked during the post-exposure bake. As a result, the latent photoacid image in the polymer became insoluble and could not be washed away in the subsequent development step.

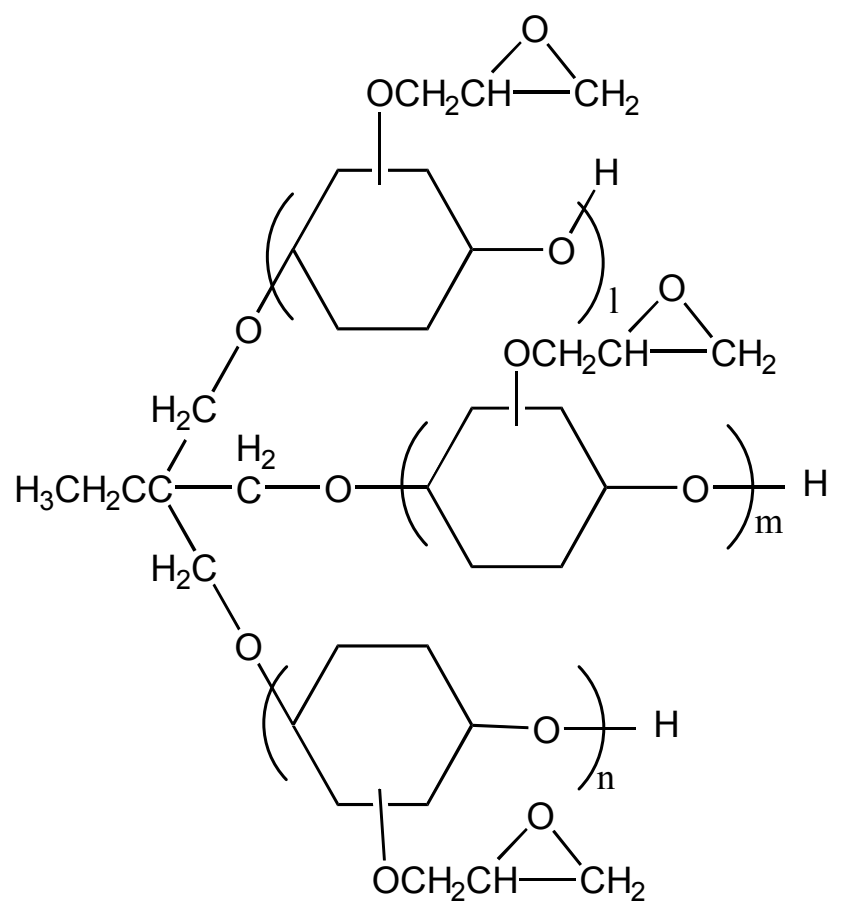

Fig. 3.4. The chemical structure of EHPE.

The recipe of the CHEP solution is follows. $3.40 \mathrm{~g}$ EHPE-3150 (Daicel) and $0.60 \mathrm{~g}$ CAPA (Aldrich) were dissolved in $6.3 \mathrm{~g}$ cyclopentanone (Aldrich) by stirring for $2 \mathrm{~h}$ at $65^{\circ} \mathrm{C}$. Afterwards $0.08 \mathrm{~g}$ UVI 6992 (Union Carbide) was added to the solution.

Polarization dependent refractive indices and thicknesses of polymer slab waveguides were measured by a prism-coupling setup (Metricon 2000), which provided measurements at wavelengths of $633 \mathrm{~nm}, 830 \mathrm{~nm}, 1300 \mathrm{~nm}$, and $1550 \mathrm{~nm}$. The measurement accuracy of the presented thickness and refractive index values are below $10 \%$ and $5 \times 10^{-4}$, respectively. The measured difference between TE and TM 
refractive indices in polymer layers are below $5 \times 10^{-4}$, which indicates that the layers are amorphous and have low birefringence. Based on the measured refractive indices at single wavelengths, the wavelength-dependent refractive index curves were fitted by Sellmeier dispersion model [112].

Figure 3.5 displays the wavelength-dependent refractive indices of 6FDA/UVR core and CHEP cladding layers by prism coupling measurements at TE polarization and Sellmeier fitting. While CHEP has a relatively low refractive index $(\mathrm{n} \sim 1.51$ at $633 \mathrm{~nm})$, the $\mathrm{Nd}^{3+}$-complex-doped material 6-FDA/UVR has a higher refractive index $(\sim 1.53$ at $633 \mathrm{~nm})$, making these two materials suitable as cladding and core materials, respectively, of rare-earth-ion complex-doped polymer waveguide structures. In this way, $\mathrm{Nd}^{3+}$-complex-doped polymer channel waveguides can be realized by a simple and reproducible procedure. Moreover, a fluorinated core material as host for the $\mathrm{Nd}^{3+}$-complex could now be applied. The difference of the refractive index between the undoped $\mathrm{Nd}^{3+}$-complex-doped 6-FDA/UVR layers is negligible.

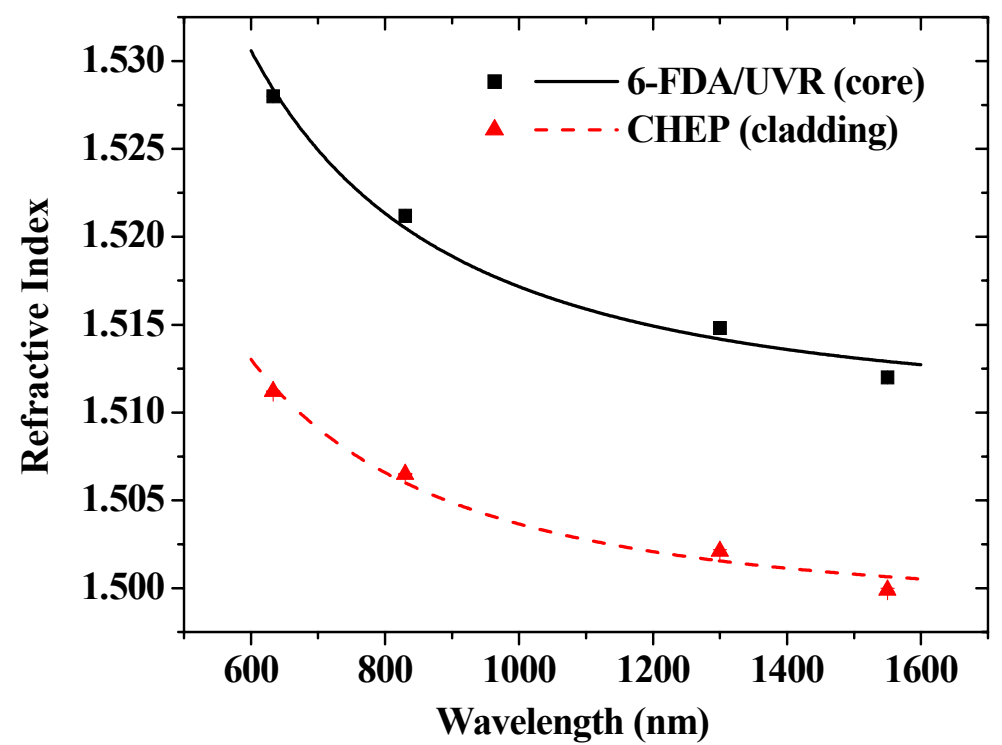

Fig. 3.5. Measured (dots) and fitted (line) refractive indices of 6-FDA/UVR and CHEP versus wavelength at TE polarization.

In order to investigate the thickness uniformity across the wafer in the spincoated polymer layers, the layer thicknesses of the two polymer films were measured as a function of distance from the center of the wafer. In general, the thickness of the polymer layers can be varied by changing the concentration of the polymer solution and speed of spin-coating. For each polymer, two layers with different thicknesses were measured with prism-coupling setup at $633 \mathrm{~nm}$ and the results are given in Fig. 3.6. The layer thickness was found to decrease by 0.31 and $1.1 \mu \mathrm{m}$ at a radius of 3.75 $\mathrm{cm}$ from the center of the wafer in a 4.4- and 8.0- $\mu \mathrm{m}$-thick (thickness measured in the center) 6-FDA/UVR layer, respectively. While in CHEP layers, the decrease of the layer thickness from the center of the wafer is $9.6 \%$ and $9.9 \%$ in a $3.49-$ and $4.90-\mu \mathrm{m}-$ thick layer, respectively. 


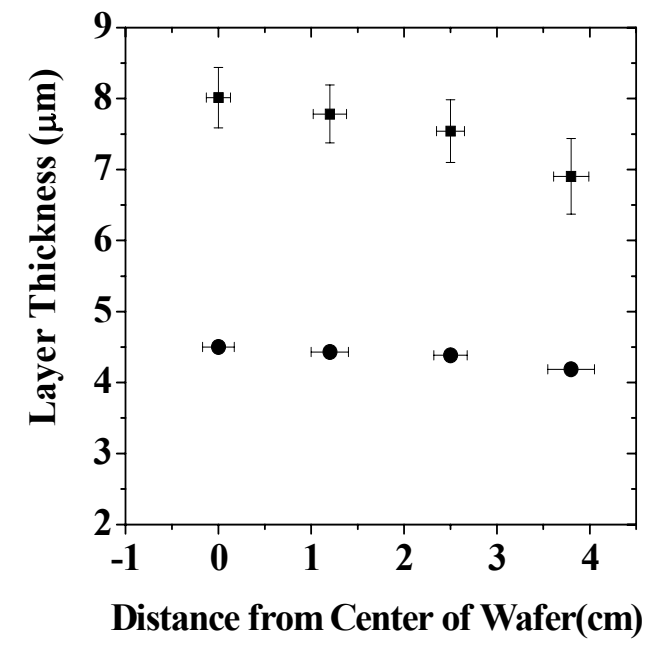

(a)

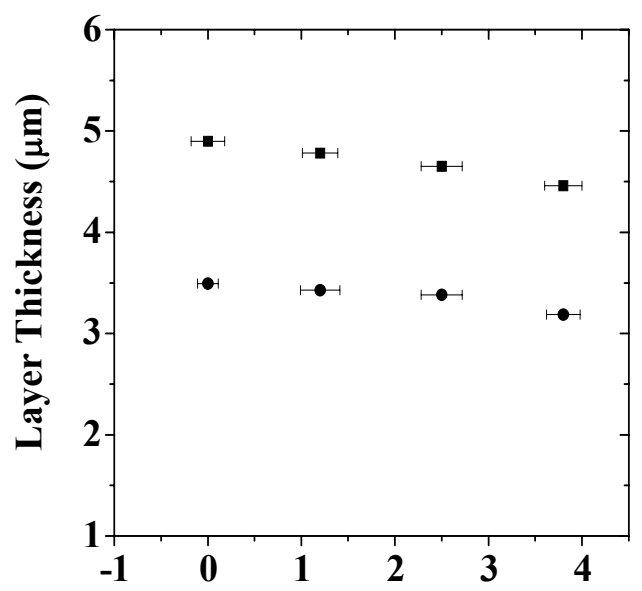

Distance from Center of Wafer(cm)

(b)

Fig. 3.6. The layer thickness of (a) 6-FDA/UVR and (b) CHEP as a function of distance from the center of the wafer.

Figure 3.7 indicates the fabrication process of $\operatorname{Nd}(\mathrm{TTA})_{3}$ phen-doped polymer channel waveguides. Thermally oxidized silicon wafers were used as substrates, with the $\sim 8-\mu \mathrm{m}$-thick oxide layer acting as the lower cladding layer. In the first approach, a 40- $\mu$ m-thick CHEP layer was spin-coated onto the oxidized silicon wafer and was used as side-cladding. (Fig. 3.7(a)). After UV exposure (350 W, I-line) through a mask with 40- $\mu$ m-wide dark lines (Fig. 3.7(b)) and subsequent curing and development, inverted channels were obtained (Fig. 3.7(c)). The core material, $\mathrm{Nd}^{3+}$ complex-doped 6-FDA/UVR, was then backfilled via spin-coating twice to achieve a smooth top wall after thermal curing (Fig. 3.7(d) and 3.7(e)). As a result, $40 \times 40-\mu \mathrm{m}^{2}$ $\mathrm{Nd}^{3+}$-complex-doped polymer channels were obtained. There was a thin $\mathrm{Nd}^{3+}$ complex-doped 6-FDA/UVR layer (of thickness of $\sim 2-4 \mu \mathrm{m}$ ) left on the top of the CHEP side-cladding after spin-coating. Since the layer is much thinner than the channel waveguides and does not have effect on light guiding, it is not necessary to remove it and can be ignored. A silicon containing epoxy with a lower refractive index of 1.49 at $633 \mathrm{~nm}$ was used as the upper cladding after UV exposure to achieve highly-multimode waveguides, and on the top of which a Pyrex glass wafer was applied to improve the quality of dicing and protect the structure (Fig. 3.7(f)).

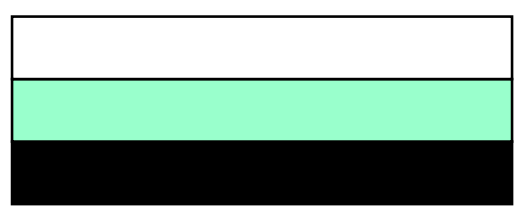

(a)

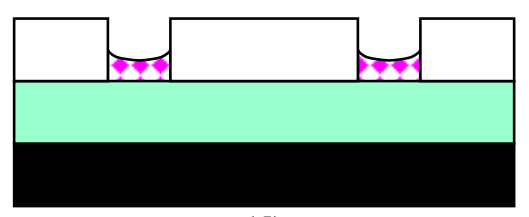

(d) 


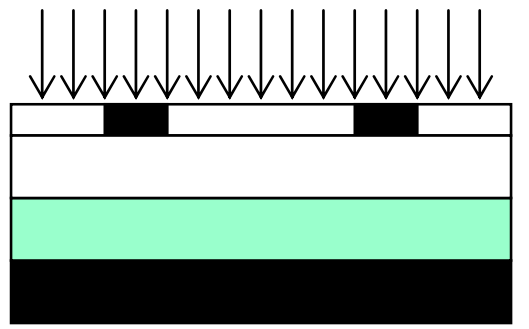

(b)

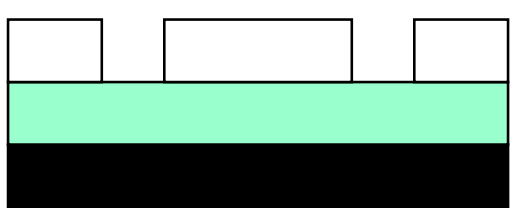

(c)

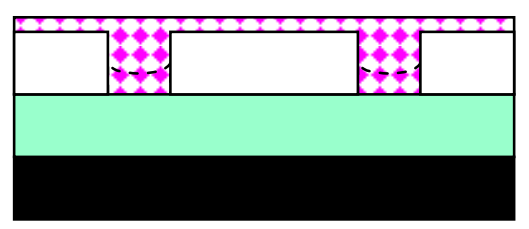

(e)

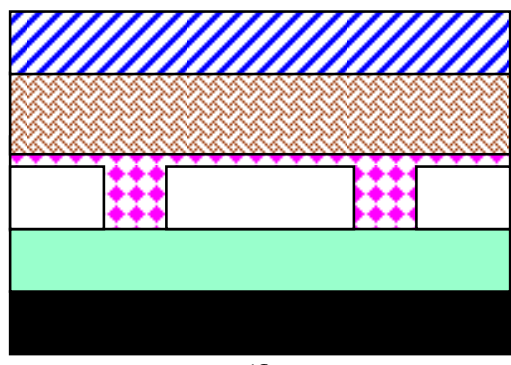

(f)

\section{Nd(TTA) ${ }_{3}$ phen-doped 6-FDA/UVR}

\section{Si-epoxy}

Pyrex wafer

Fig. 3.7. Fabrication process of $\mathrm{Nd}^{3+}$-complex-doped polymer channel waveguides. (a) spin-coating CHEP layer onto a thermally oxidized Si wafer; (b) UV exposure; (c) developing in RER 600; (d) and (e) backfilling core solution twice; (f) UV exposure of Si-epoxy upper cladding layer and Pyrex wafer.

Figure 3.8(a) is the top-view microscope picture of $40 \times 40-\mu \mathrm{m}^{2} \mathrm{Nd}^{3+}$-doped channel after backfilling the core material and Figure 3.8(b) shows a microscope picture of the cross section of the waveguide.

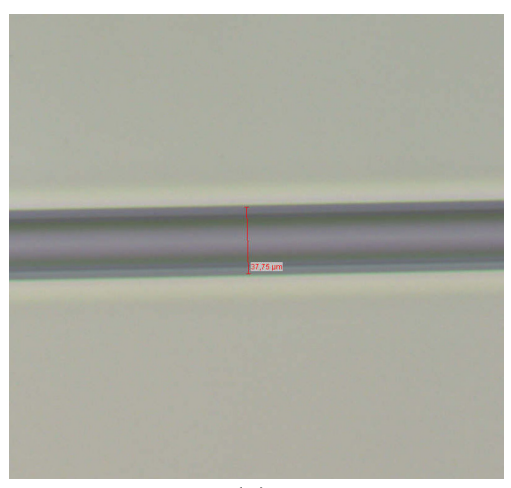

(a)

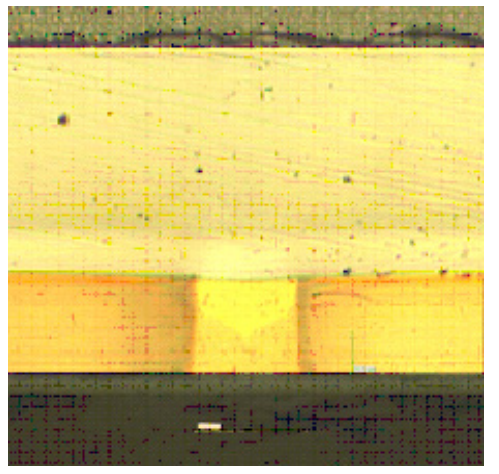

(b)

Fig. 3.8. Microscope pictures of $\mathrm{Nd}^{3+}$-complex-doped polymer $40 x 40-\mu \mathrm{m}^{2}$ channel waveguides: (a) top view without top-cladding; (b) cross section.

As the excitation fraction of $\mathrm{Nd}^{3+}$ ions in the excited state is strongly dependent on the excitation intensity, a smaller cross section of the channel is needed for achieving optical gain in the channel waveguides with $\mathrm{Nd}^{3+}$ dopants using reasonable pump powers. Therefore, in the second approach $5 \times 5-\mu \mathrm{m}^{2} \mathrm{Nd}^{3+}$-complexdoped polymer channels were fabricated based on a similar fabrication process as that 
of $40 \times 40-\mu \mathrm{m}^{2}$ channels. The only difference was that, instead of using a thick Si-

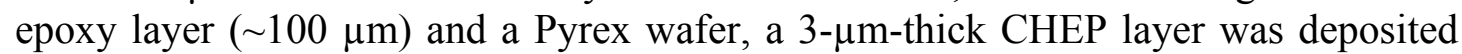
on top of the channels as the upper cladding layer by spin-coating and thermal curing. The geometry of the resulting structure together with a microscope image of the cross section of a channel waveguide is shown in Fig. 3.9. The $\mathrm{Nd}^{3+}$-complex-doped layer left on the surface of the CHEP side cladding after backfilling of the channels is sufficiently thin $(\sim 0.5-1 \mu \mathrm{m})$ that it can be ignored. The waveguide can be shortened by dicing with attaching a small piece of glass on the top or cleaving directly.

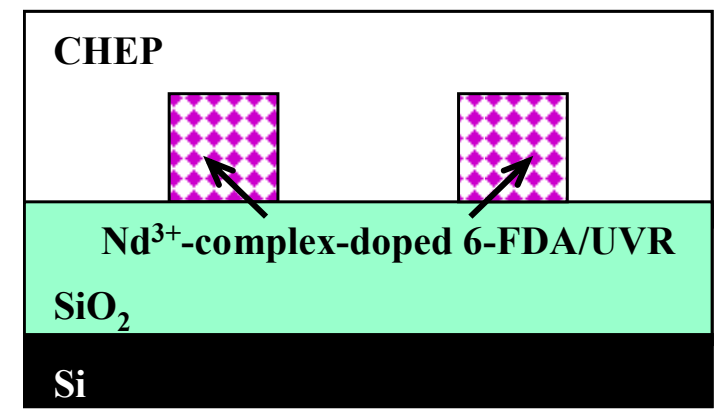

(a)

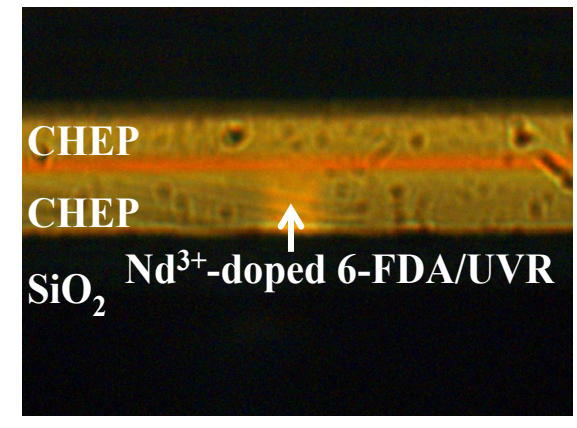

(b)

Fig. 3.9. (a) Geometry and (b) optical microscope cross section picture of the $5 \times 5-\mu \mathrm{m}^{2} \mathrm{Nd}^{3+}$-complexdoped polymer channel waveguides.

In conclusion, by dividing the functionalities of photo-definition and active doping over two different polymers, $\mathrm{Nd}^{3+}$-complex-doped, photo-defined polymer channel waveguides were realized on thermally oxidized silicon wafers with a simple and reproducible fabrication procedure.

\section{3 $\mathrm{Al}_{2} \mathrm{O}_{3}: \mathrm{Nd}^{3+}$ Waveguide Fabrication}

Amorphous aluminum oxide, which has high mechanical stability and thermal conductivity, doped with rare-earth ions is a very attractive material for active integrated optical applications such as amplifiers and lasers. Its relatively high refractive index allows for the realization of high-contrast channel waveguides with considerably more compact IO circuits compared to many polymers and other glasses. Furthermore, it is compatible with Si-technology.

\subsubsection{Film Deposition}

The deposition of $\mathrm{Al}_{2} \mathrm{O}_{3}$ layer with Er dopants by reactive co-sputtering has been well developed in the IOMS group in previous work [41, 42], and has been applied to the deposition of $\mathrm{Al}_{2} \mathrm{O}_{3}: \mathrm{Nd}^{3+}$ films.

For the $\mathrm{Al}_{2} \mathrm{O}_{3}$ layer growth, an AJA ATC 1500 sputtering system equipped with a load-lock and three sputtering guns was used. A schematic illustration of the sputtering system is displayed in Fig. 3.10. The sample was fixed in a bottom-up sputtering configuration on a substrate holder which can be rotated and heated up to a maximum temperature of $800^{\circ} \mathrm{C}$. The deposition chamber can be pumped to a background pressure of $10^{-7} \mathrm{mTorr}$, which is essential in order to reach a negligible $\mathrm{OH}^{-}$level in the deposition process. The three sputtering guns are designed for 2-inch 
sputtering targets and can be driven individually by RF or DC power supplies, having a maximum range of $500 \mathrm{~W}$. With respect to the optical loss of grown layers, the RF sputtering was chosen and process parameters were optimized.

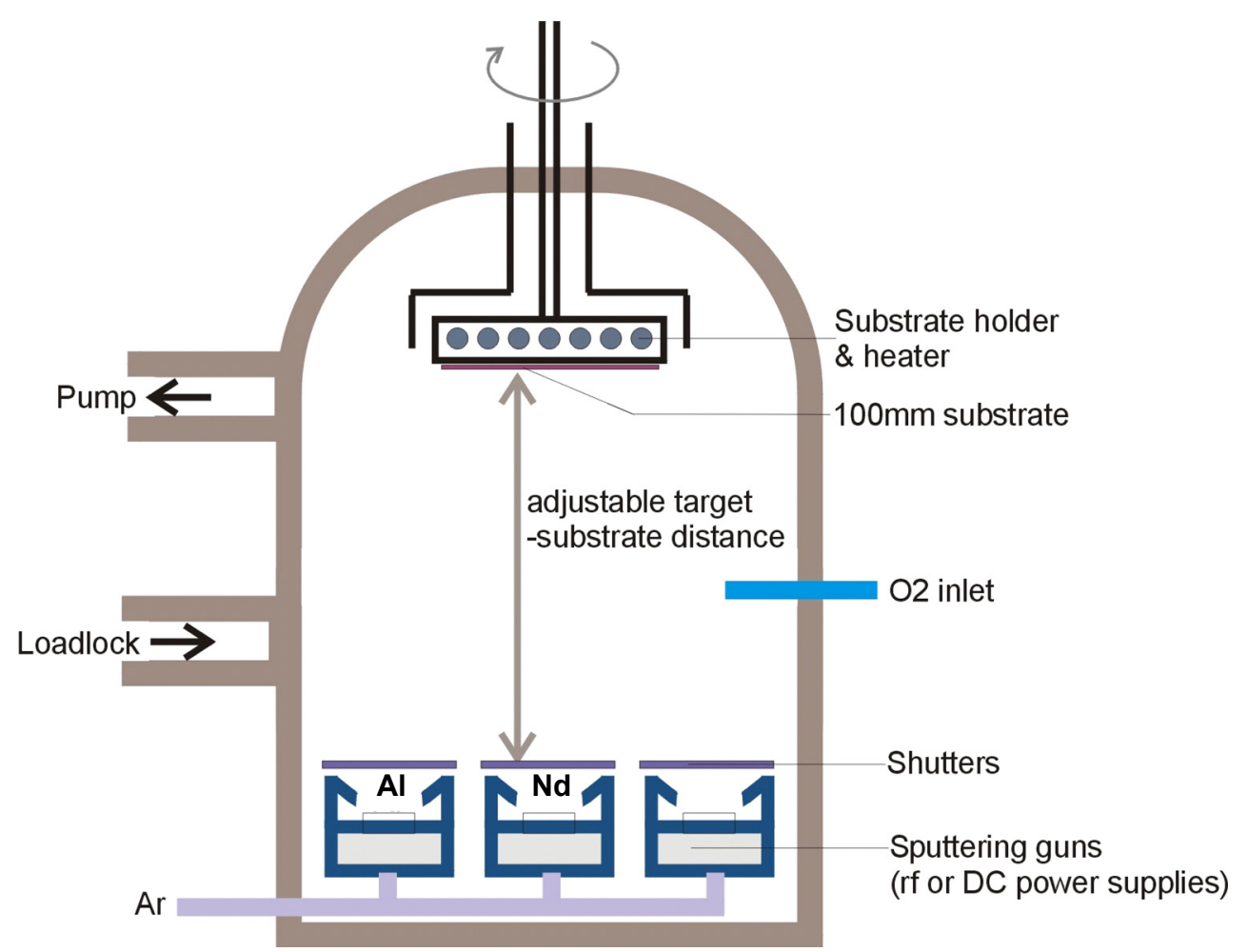

Fig. 3.10. Schematic illustration of the reactive co-sputtering system used for the $\mathrm{Al}_{2} \mathrm{O}_{3}: \mathrm{Nd}^{3+}$ film growth.

High purity metallic Al (99.999\% purity) and Nd (99.95\% purity) targets were sputtered separately using Ar guns, while oxygen was supplied as a gas. The sputtering power applied to the Al target was held constant at $200 \mathrm{~W}$, while the Ndtarget power was varied between 9 to $19 \mathrm{~W}$ in order to vary the $\mathrm{Nd}^{3+}$ concentration. The $\mathrm{Nd}^{3+}$ concentration, which is uniform throughout the layer, was determined by Rutherford Backscattering Spectroscopy (RBS) at the University of Utrecht. Figure 3.11 shows the resulting $\mathrm{Nd}^{3+}$ concentration versus sputtering power applied to the $\mathrm{Nd}$ target.

The refractive index and thickness of the deposited layers were measured by the prism coupling method (Metricon). Since all the layers are amorphous and yield low stress values, the birefringence is low and the difference between TE and TM refractive indices were measured to vary between 5 and $10 \times 10^{-4}$. Figure 3.12(a) shows the wavelength-dependent refractive indices of an $\mathrm{Al}_{2} \mathrm{O}_{3}: \mathrm{Nd}^{3+}$ and $\mathrm{Al}_{2} \mathrm{O}_{3}$ layer measured at $633 \mathrm{~nm}, 830 \mathrm{~nm}, 1300 \mathrm{~nm}$, and $1550 \mathrm{~nm}$ and fitted by the Sellmeier model. The refractive index of the layer doped with $\mathrm{Nd}^{3+}$ is slightly higher than that of the non-doped layer. The thickness uniformity of $\mathrm{Al}_{2} \mathrm{O}_{3}: \mathrm{Nd}^{3+}$ layers was studied, and the refractive indices of two layers as a function of distance from the center of the wafer are given in Fig 3.12(b). The decrease of the layer thickness from the center to the edge of the wafer in a $1.0-$ and $4.8-\mu \mathrm{m}$-thick layer is 0.16 and $0.52 \mu \mathrm{m}$, respectively, which gives a reduction of $\sim 10 \%$. 


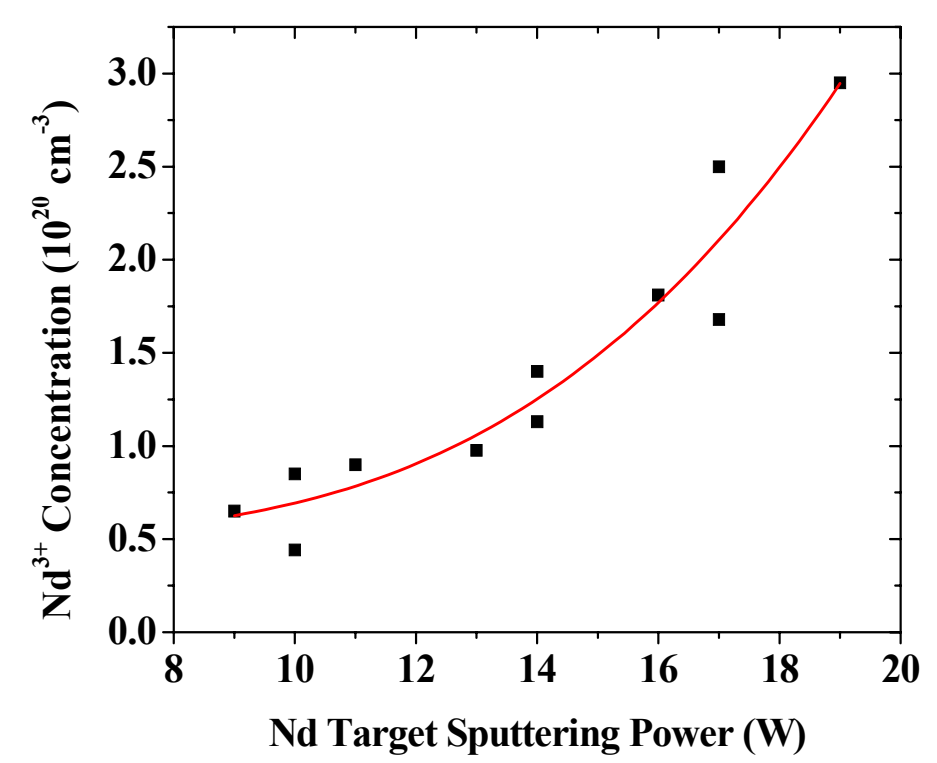

Fig. 3.11. $\mathrm{Nd}^{3+}$ concentration vs. Nd target sputtering power (dots) and polynomial fit (line).

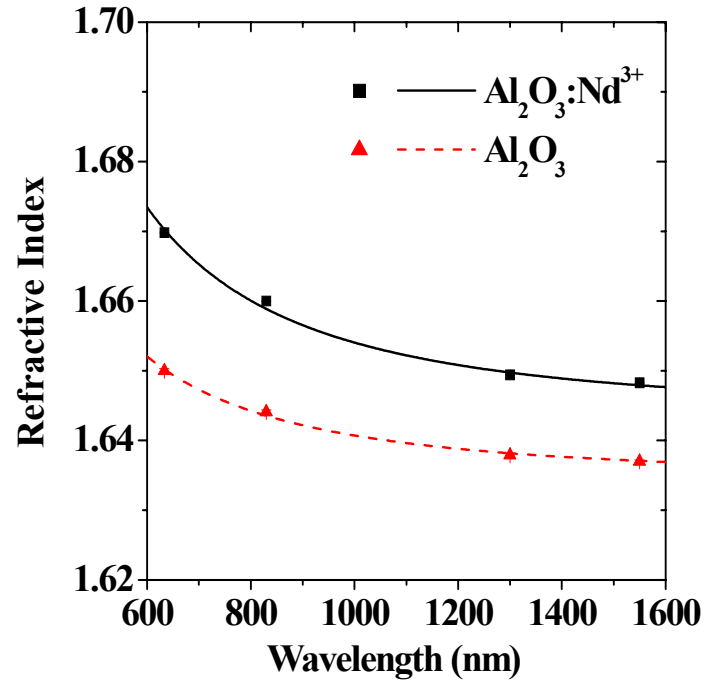

(a)

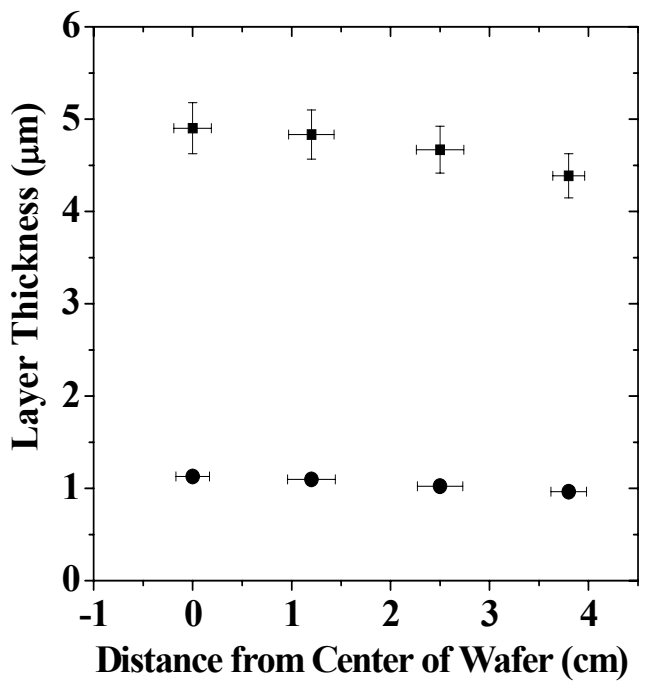

(b)

Fig. 3.12. (a) Measured (dots) and fitted (line) refractive indices of an $\mathrm{Al}_{2} \mathrm{O}_{3}: \mathrm{Nd}^{3+}$ and $\mathrm{Al}_{2} \mathrm{O}_{3}$ layer; (b) the layer thickness of two $\mathrm{Al}_{2} \mathrm{O}_{3}: \mathrm{Nd}^{3+}$ layers as a function of distance from the center of the wafer.

\subsubsection{Waveguide Structuring}

In order to define channel waveguides in $\mathrm{Al}_{2} \mathrm{O}_{3}$ films, the etching behaviour of $\mathrm{Al}_{2} \mathrm{O}_{3}$ has been investigated by Bradley et al. using an inductively coupled reactive ion etch (RIE) system [40-42]. The etch rate of $\mathrm{Al}_{2} \mathrm{O}_{3}$ and possible mask materials were studied by applying various common process gases and combinations of these gases, including $\mathrm{CF}_{4} / \mathrm{O}_{2}, \mathrm{BCl}_{3}, \mathrm{BCl}_{3} / \mathrm{HBr}$ and $\mathrm{Cl}_{2}$. Based on a comparison of the etch rates and patterning feasibility of the different mask materials, a $\mathrm{BCl}_{3} / \mathrm{HBr}$ plasma and standard resist mask were used to fabricate channel waveguide structures. The etched structures exhibit straight sidewalls with minimal roughness and etch depths of up to 1 
$\mu \mathrm{m}$, sufficient for defining waveguides with strong optical confinement and low bending losses. Low additional propagation losses $(\sim 0.1 \mathrm{~dB} / \mathrm{cm}$ at $1523 \mathrm{~nm})$ were measured in single-mode $\mathrm{Al}_{2} \mathrm{O}_{3}$ ridge waveguides defined using the developed etching process.

Figure 3.13 indicates the fabrication process of $\mathrm{Al}_{2} \mathrm{O}_{3}: \mathrm{Nd}^{3+}$ channel waveguides, which follows the etching receipt of Bradley. $1.5 \mu \mathrm{m}$ thick resist was deposited on an $\mathrm{Al}_{2} \mathrm{O}_{3}$ layer (Fig. 3.13(a)) by spin-coating (Fig. 3.13(b)) and patterned using standard lithography (Fig. 3.13(c)). Etching of the $\mathrm{Al}_{2} \mathrm{O}_{3}$ layer was carried out using an Oxford Plasmalab 100 inductively-coupled plasma (ICP) RIE system (Fig. 3.13(d)). The optimized gas ratio in terms of selectivity for the amorphous $\mathrm{Al}_{2} \mathrm{O}_{3}$ films was found to be $5: 2\left(\mathrm{BCl}_{3}: \mathrm{HBr}\right)$ and optimized process parameters were ICP power $=1750 \mathrm{~W}, \mathrm{RF}$ electrode power $=25 \mathrm{~W}$, pressure $=12 \mathrm{mTorr}$, and a total gas flow of $35 \mathrm{sccm}$.

In order to characterize the refractive index, layer thickness, and loss, one half of the layers were left unpatterned for planar waveguide experiments. Two types of $\mathrm{Al}_{2} \mathrm{O}_{3}: \mathrm{Nd}^{3+}$ channel waveguides were designed and fabricated for single-mode and multi-mode waveguide amplifiers.

The $\mathrm{Al}_{2} \mathrm{O}_{3}: \mathrm{Nd}^{3+}$ channels without top-cladding layer in Fig. 3.13(e) have a width of $2.0 \mu \mathrm{m}$ and shallow etch-depth of $\sim 70 \mathrm{~nm}$, using air as the cladding. The etch depth, layer thickness, and waveguide width were selected to ensure strong confinement of the propagating optical signal within the $\mathrm{Al}_{2} \mathrm{O}_{3}: \mathrm{Nd}^{3+}$ layers and single-mode behaviour at wavelengths longer than $850 \mathrm{~nm}$, with excellent overlap of signal and pump modes. In this Chapter, all the characterizations of the $\mathrm{Al}_{2} \mathrm{O}_{3}: \mathrm{Nd}^{3+}$ channel waveguides are based on such waveguide geometry.

For multi-mode waveguide amplifiers and devices, the $\mathrm{Al}_{2} \mathrm{O}_{3}: \mathrm{Nd}^{3+}$ layers

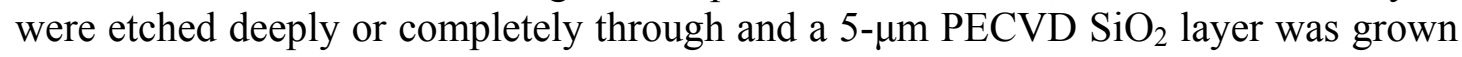
on top (Fig. 3.13(f)). The details of the waveguide characterization will be discussed in Chapter 5.

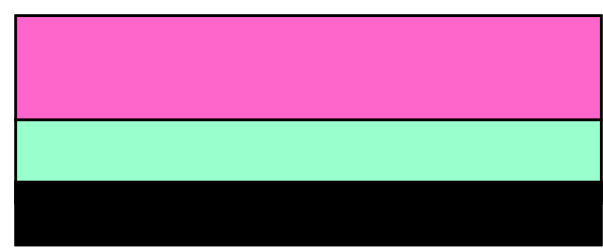

(a)

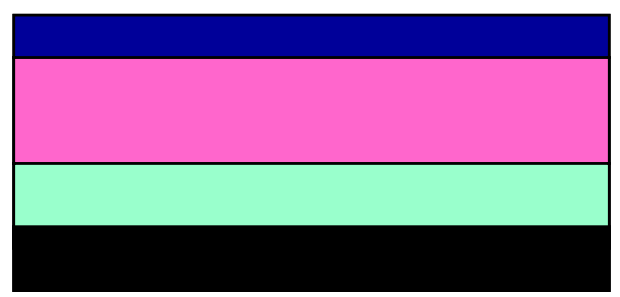

(c)

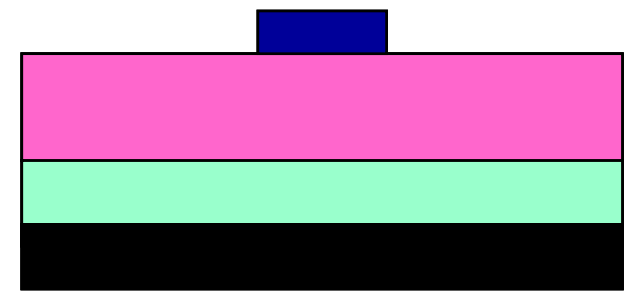

(b)

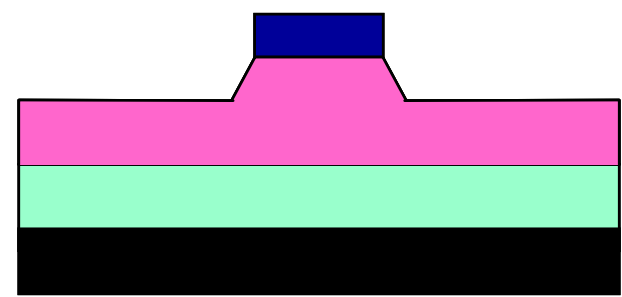

(d) 


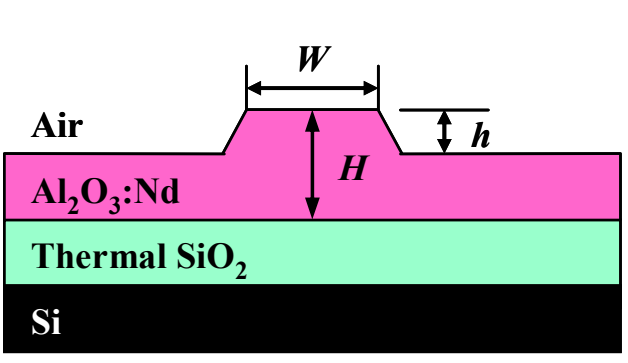

(e)

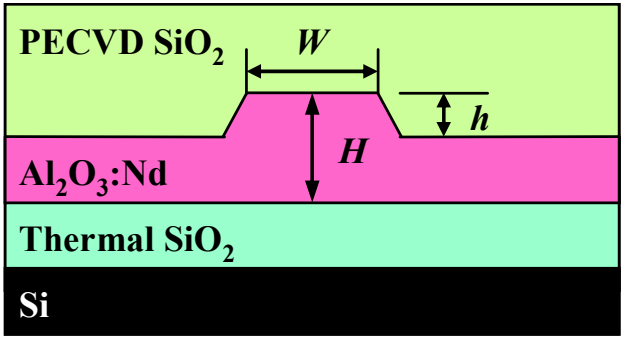

(f)

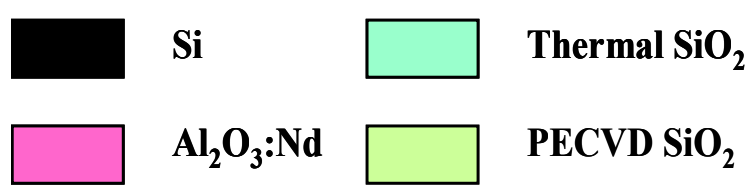

Fig. 3.13. Fabrication process of $\mathrm{Al}_{2} \mathrm{O}_{3}: \mathrm{Nd}^{3+}$ channel waveguides. (a) Reactive co-sputtering of an $\mathrm{Al}_{2} \mathrm{O}_{3}: \mathrm{Nd}^{3+}$ film on a thermally oxide Si substrate; (b) spin-coating resist; (c) pattering resist by standard lithography; (d) Etching $\mathrm{Al}_{2} \mathrm{O}_{3}: \mathrm{Nd}^{3+}$ in Oxford Plasmalab ICP RIE; (e) channel waveguide without top-cladding; (f) channel waveguide with PECVD top-cladding.

\subsection{Optical Losses}

\subsubsection{Optical Attenuation and Loss Measurement}

Before proceeding with waveguide amplifiers and lasers, it was important to demonstrate low propagation losses in the channel waveguides. Here, the theory and measurement methods related to optical loss characterization in waveguides is presented.

The intensity of light propagating through a medium can be attenuated due to several mechanisms, e.g. scattering and material absorption. The reduction of light intensity $I$ at a wavelength $\lambda$, when propagating through a medium of short length $d z$ (Fig. 3.14) can be described by the Lambert-Beer law [74]:

$$
\frac{d I}{d z}=-\mathrm{a}(\lambda) I
$$

where $\mathrm{a}(\lambda)$ is the wavelength-dependent loss coefficient (in $\mathrm{cm}^{-1}$ ).

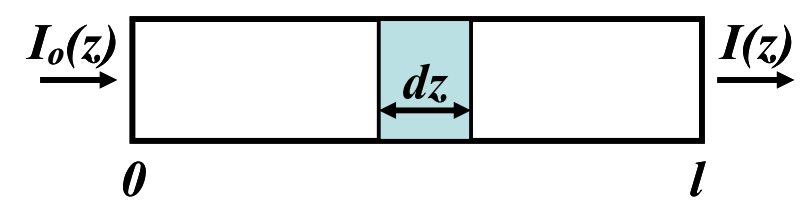

Fig. 3.14. Schematic of light propagating through an optical material.

Solving this differential equation for a propagation length $l$ (in $\mathrm{cm}$ ) results in: 


$$
I=I_{0} \cdot \exp (-\mathrm{a}(\lambda) \cdot l)
$$

Extraction of the loss coefficient a $(\lambda)$ yields:

$$
\mathrm{a}(\lambda)=\frac{1}{l} \cdot \ln \left(\frac{I}{I_{0}}\right)
$$

Often when describing the propagation losses in optical waveguides, the absorption coefficient is given in units of $\mathrm{dB} / \mathrm{cm}$. In this case the absorption coefficient is written as $\alpha(\lambda)$ and related to $\mathrm{a}(\lambda)$ by:

$$
\alpha(\lambda)=\frac{-10 \cdot \log \left(I / I_{0}\right)}{l}=10 \cdot \log e \cdot \mathrm{a} \approx 4.34 \cdot \mathrm{a}
$$

We will consistently use the parameter $\alpha(\lambda)$ when we discuss optical losses in waveguides. The total loss in a waveguide is the combination of the different loss mechanisms, and we mainly consider the following losses in this thesis:

$$
\alpha_{\text {total }}(\lambda)=\alpha_{\text {mat }}(\lambda)+\alpha_{\text {scat }}(\lambda)+\alpha_{a b s}(\lambda)
$$

where $\alpha_{\text {mat }}(\lambda)$ is the material-dependent background loss, $\alpha_{\text {scat }}(\lambda)$ is the loss due to scattering at the core-cladding interface or roughness of the waveguide sidewalls, and $\alpha_{a b s}(\lambda)$ is the loss due to absorption by $\mathrm{Nd}^{3+}$ ions. Optical measurements are required to determine each of these contributions to the total propagation loss of optical waveguides.

Optical losses of $\mathrm{Nd}^{3+}$-complex-doped polymer and $\mathrm{Al}_{2} \mathrm{O}_{3}: \mathrm{Nd}^{3+}$ waveguides have been investigated in both slab and channel structures. In general, the wavelength-dependent optical loss can be determined by measuring the transmitted light coupled out from a waveguide with different propagation lengths $l_{\mathrm{n}}(\mathrm{n}=0,1$, $2 \ldots)$. By a least-squares fit of a set of measured transmitted light intensities at a certain wavelength or transmitted spectra with Eq. 3.2 or 3.3, the single-wavelength loss or loss spectrum of a film waveguide can be determined.

A prism coupling method was applied to determine the optical loss of slab waveguides, and the experimental setup is depicted in Fig. 3.15. A light source is coupled into the film and coupled out after propagating a certain distance through the film using two prisms. The propagation distance $l$ in the film is varied by fixing the position of the in-coupling prism while moving the out-coupling prism. A large-core liquid guiding fiber, which is connected to a detector, is used to collect the transmitted light with different propagation lengths from the out-coupling prism. 


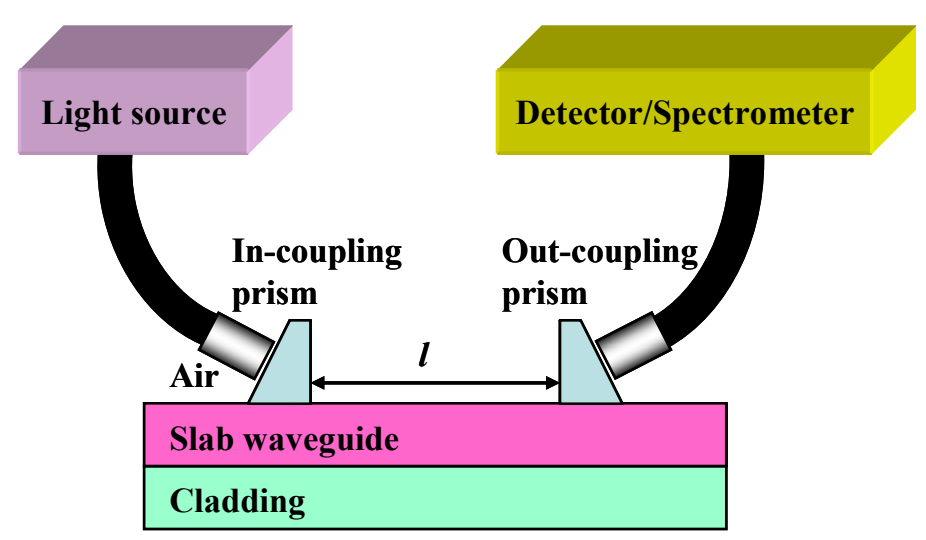

Fig. 3.15. Prism coupling setup used for propagation loss measurements in slab waveguides.

The cut-back method is a widely used approach for loss investigations in channel waveguides. The output from a light source is coupled into a channel waveguide and the transmitted light is coupled out by an optical fiber (see Fig. 3.16). The out-coupled transmitted light from the channel waveguide is then collected by a detector. The loss of the waveguide is determined from the power of transmitted light recorded for waveguides with different channel lengths cut from a single sample, which were otherwise identical. Assuming that the input- and output-coupling of fibers remain constant in a series of measurements for samples with different propagation length, the waveguide loss $\alpha(\lambda)$, can be obtained.

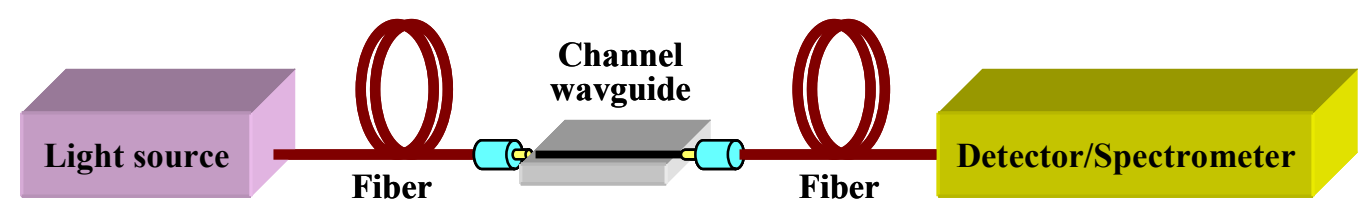

Fig. 3.16. Fiber coupling setup for cut-back loss measurement in a channel waveguide.

For determining the loss of a channel waveguide at a certain wavelength, a single-wavelength light source at the desired wavelength is coupled into the waveguide and the intensity of the transmitted light with various propagation lengths is collected by a photo detector $(\mathrm{Si} / \mathrm{Ge} / \mathrm{InGaAs})$, based on which the optical loss at this wavelength can be determined. For a single-mode waveguide, a polarized light source is used for measuring the optical loss at TE and TM polarization.

To obtain the loss spectrum at a certain wavelength range, the output from a broadband fiber-coupled white-light source (FemtoPower1060, SC450, Fianium) is launched into waveguides of varying lengths and a set of transmitted spectra is then recorded by a spectrometer (iHR550, Horiba, Jobin Yvon). The spectra were measured independent of polarization. By fitting the transmission spectra and calibrating with the measured loss at a single wavelength averaged with TE and TM polarization, the loss spectrum is obtained. 


\subsubsection{Loss and Absorption Spectra}

Fundamental requirements imposed on a host for optical gain and lasing provided by rare-earth dopant ions are high optical quality with a low level of scattering loss and a wide transparency range that covers both the pump and lasing wavelengths.

$\mathrm{Nd}^{3+}$ ions are suitable dopants for optical polymers, as many polymers exhibit very low loss at the infrared wavelength range, especially at the 1060-nm laser transition. The optical loss of the polymer host used in this work, 6-FDA/UVR, was first investigated in polymer films with and without $\mathrm{Nd}^{3+}$ complexes by prism coupling measurement and the results are given in Fig. 3.17. The polymer host, 6FDA/UVR, shows a very broad transmission band in the wavelength range 550-1150 $\mathrm{nm}$, which indicates the low yellowing effect in this material. The $\mathrm{Nd}^{3+}$ concentration in the doped polymer film (Fig. 3.17(b)) was $0.11 \times 10^{20} \mathrm{~cm}^{-3}$. The absorption peaks near 1200 and $1400 \mathrm{~nm}$ are due to vibrational modes of remaining $\mathrm{C}-\mathrm{H}$ bonds in the polymer host $[29,30]$. The absorption peaks at $580 \mathrm{~nm}, 745 \mathrm{~nm}, 800 \mathrm{~nm}$, and $870 \mathrm{~nm}$ in Fig. 3.17(b) are from the absorption transitions of $\mathrm{Nd}^{3+}$ ions.

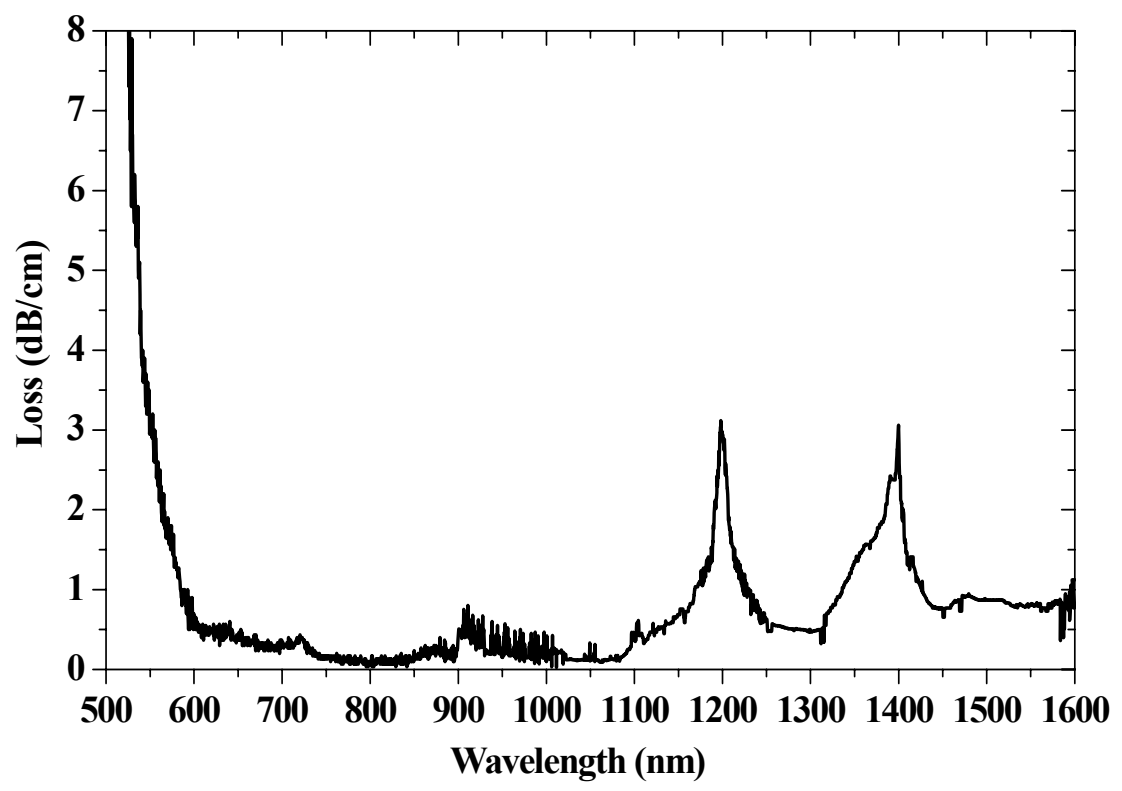

(a) 


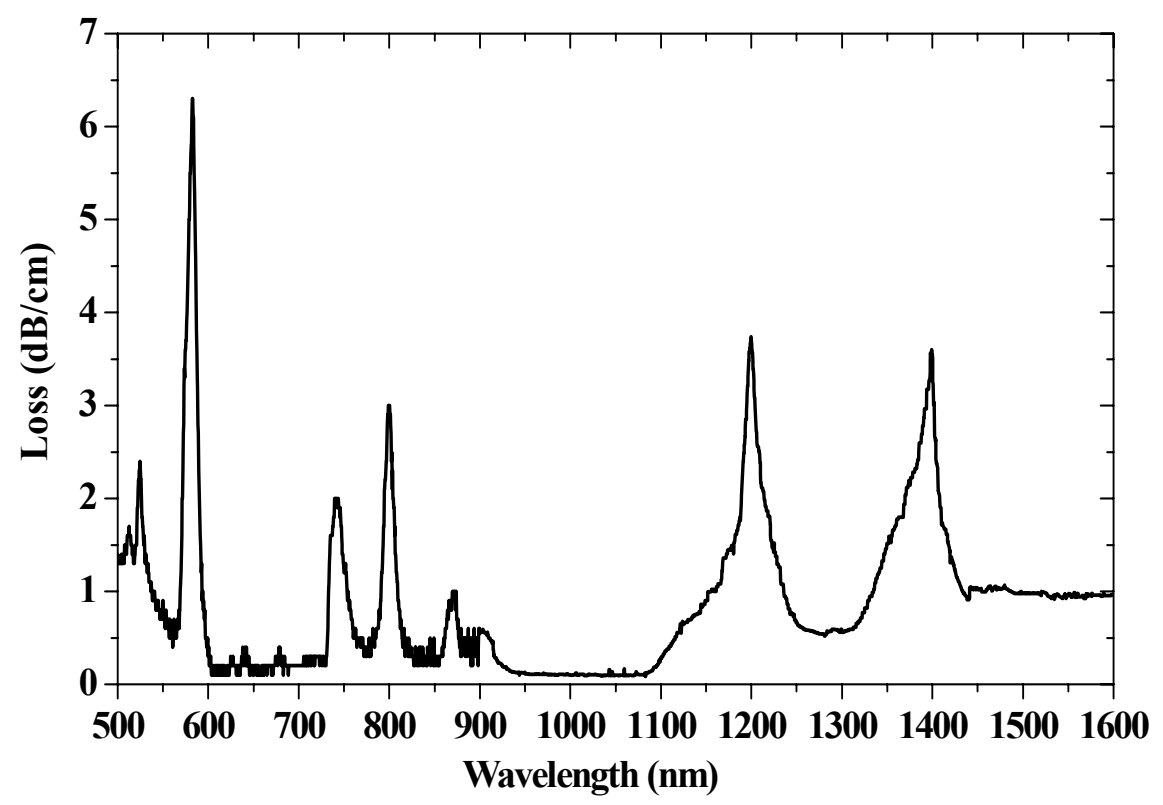

(b)

Fig. 3.17. Loss spectrum of (a) undoped and (b) $\mathrm{Nd}^{3+}$-complex-doped 6-FDA/UVR film

Losses of polymer channel waveguides were measured using the cut-back method. Fig. 3.18(a) displays the loss spectrum of a $\mathrm{Nd}^{3+}$-complex-doped large-core $\left(40 \times 40 \mu \mathrm{m}^{2}\right)$ polymer channel waveguide with $\mathrm{Nd}^{3+}$ concentration of $0.1 \times 10^{20} \mathrm{~cm}^{-3}$ with very low background propagation and scattering loss at 700-1100 nm

Small-core waveguides are preferable for achieving gain due to the lower pump power needed. Optical losses of the small-core $\mathrm{Nd}^{3+}$-complex-doped 6FDA/UVR waveguides channel waveguides at $633 \mathrm{~nm}, 830 \mathrm{~nm}, 1064 \mathrm{~nm}$, and 1330 $\mathrm{nm}$ were measured with unpolarized light due to the multi-mode waveguide structure, and the average losses at the four wavelengths are given in Table 3.1.

Table. 3.1. Optical loss (in $\mathrm{dB} / \mathrm{cm}$ ) in films and channel waveguides of $\mathrm{Nd}^{3+}$-complex-doped 6FDA/UVR and $\mathrm{Al}_{2} \mathrm{O}_{3}: \mathrm{Nd}^{3+}$ at $633 \mathrm{~nm}, 830 \mathrm{~nm}, 880 \mathrm{~nm}, 1064 \mathrm{~nm}$, and $1330 \mathrm{~nm}$.

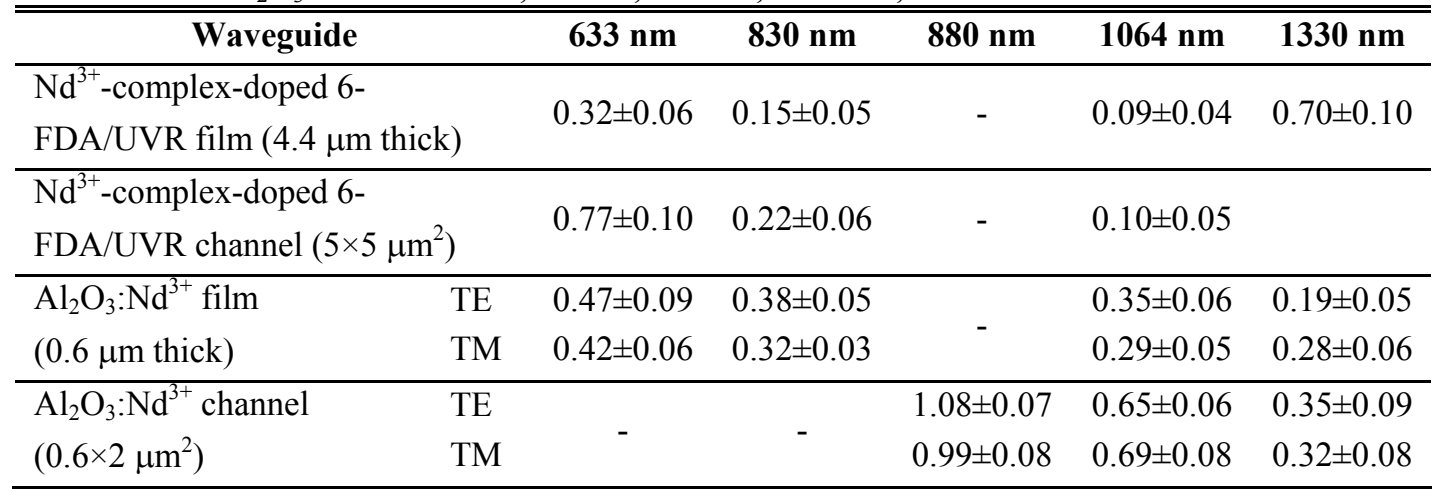

Figure 3.18 (b) displays the broadband loss spectrum of a $5 \times 5-\mu \mathrm{m}^{2} \mathrm{Nd}^{3+}$ complex-doped 6-FDA/UVR channel waveguide at the visible and infrared wavelength range calibrated by measured single-wavelength losses. The background propagation loss at $840-950 \mathrm{~nm}$ is around $0.2-0.3 \mathrm{~dB} / \mathrm{cm}$, and the value measured for the waveguide loss using this approach was $\sim 0.1 \mathrm{~dB} / \mathrm{cm}$ at $1060 \mathrm{~nm}$. 


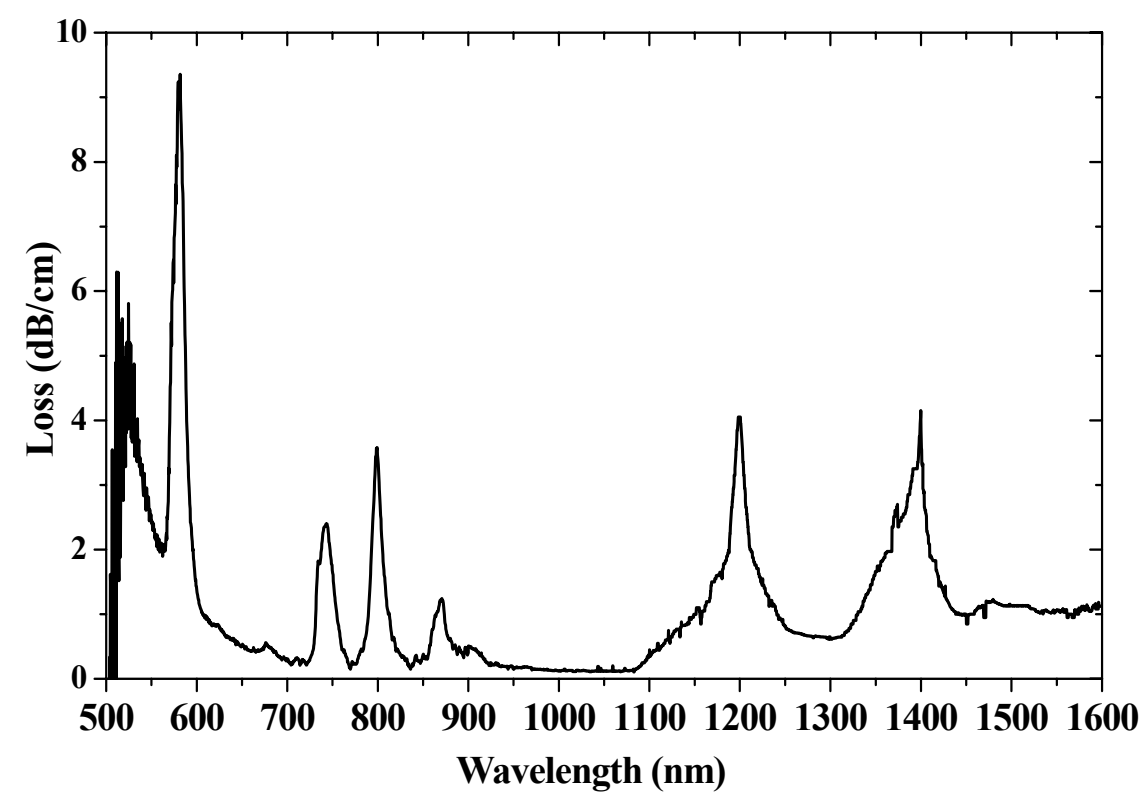

(a)

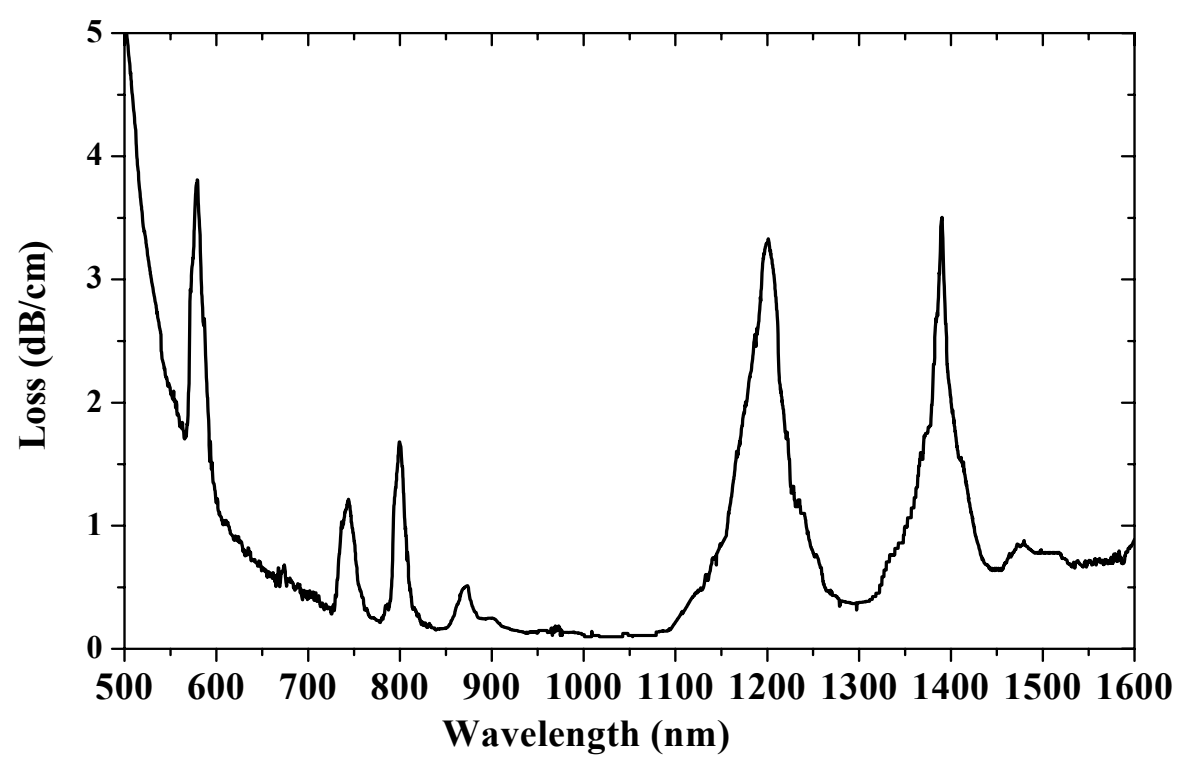

(b)

Fig. 3.18. Loss spectrum of a $\mathrm{Nd}^{3+}$-complex-doped 6-FDA/UVR channel waveguide of (a) $40 \times 40 \mu \mathrm{m}^{2}$ and (b) $5 \times 5 \mu \mathrm{m}^{2}$.

The absorption spectrum of $\mathrm{Nd}^{3+}$ in 6-FDA/UVR, depicted in Fig. 3.19(a), was obtained by subtracting the background loss from the recorded loss spectrum in Fig. 3.18(b). The absorption peaks at $580 \mathrm{~nm}, 745 \mathrm{~nm}, 800 \mathrm{~nm}$, and $870 \mathrm{~nm}$ correspond to the $\mathrm{Nd}^{3+}$ absorption transitions ${ }^{4} \mathrm{I}_{9 / 2} \rightarrow{ }^{4} \mathrm{G}_{5 / 2}+{ }^{2} \mathrm{G}_{7 / 2},{ }^{4} \mathrm{I}_{9 / 2} \rightarrow{ }^{4} \mathrm{~F}_{7 / 2}+{ }^{4} \mathrm{~S}_{3 / 2}$, ${ }^{4} \mathrm{I}_{9 / 2} \rightarrow{ }^{4} \mathrm{~F}_{5 / 2}+{ }^{2} \mathrm{H}_{9 / 2}$, and ${ }^{4} \mathrm{I}_{9 / 2} \rightarrow{ }^{4} \mathrm{~F}_{3 / 2}$, respectively. The absorption cross sections can be calculated from the measured absorption spectrum using Eq. 2.18. Considering the confinement factor $\Gamma$ of the beam in the waveguide and the relation between $\alpha$ and a (see Eq. 3.6), the absorption cross section given in Fig. 3.19(b) was calculated by the following equation: 


$$
\sigma_{a b s}(\lambda)=\frac{\alpha_{a b s}}{\log (e) \cdot N_{d} \cdot \Gamma}
$$

where $N_{d}$ is the $\mathrm{Nd}^{3+}$ concentration in the waveguide.

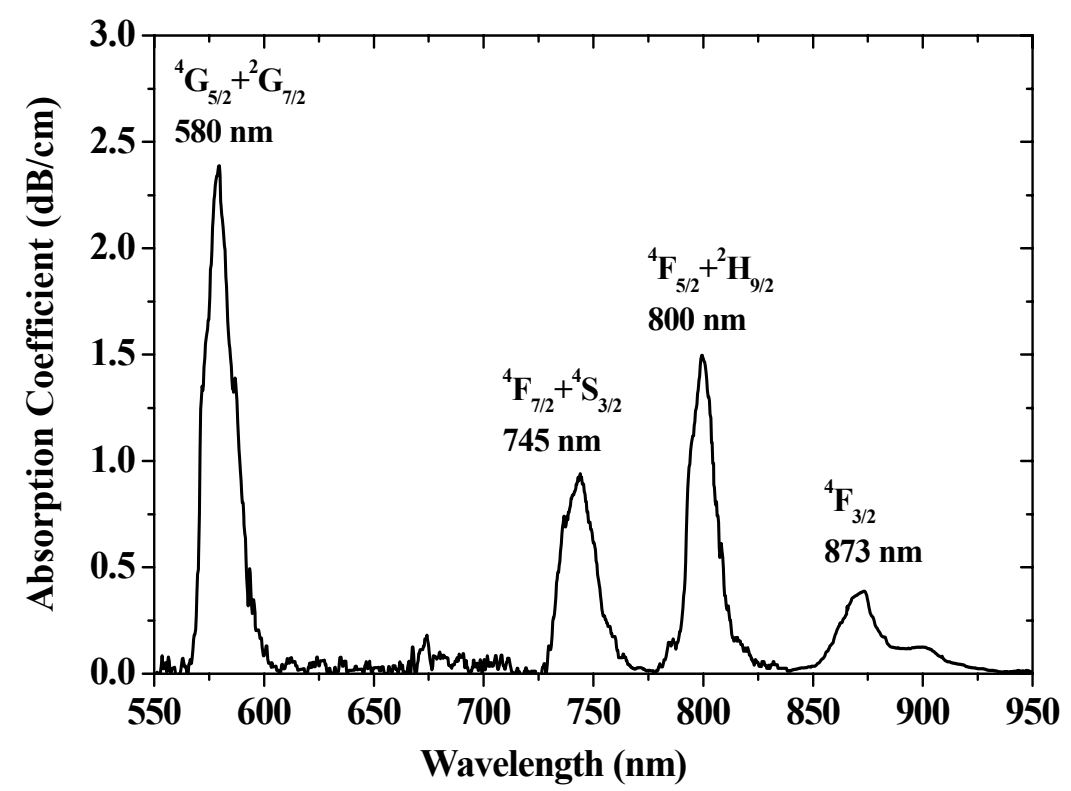

(a)

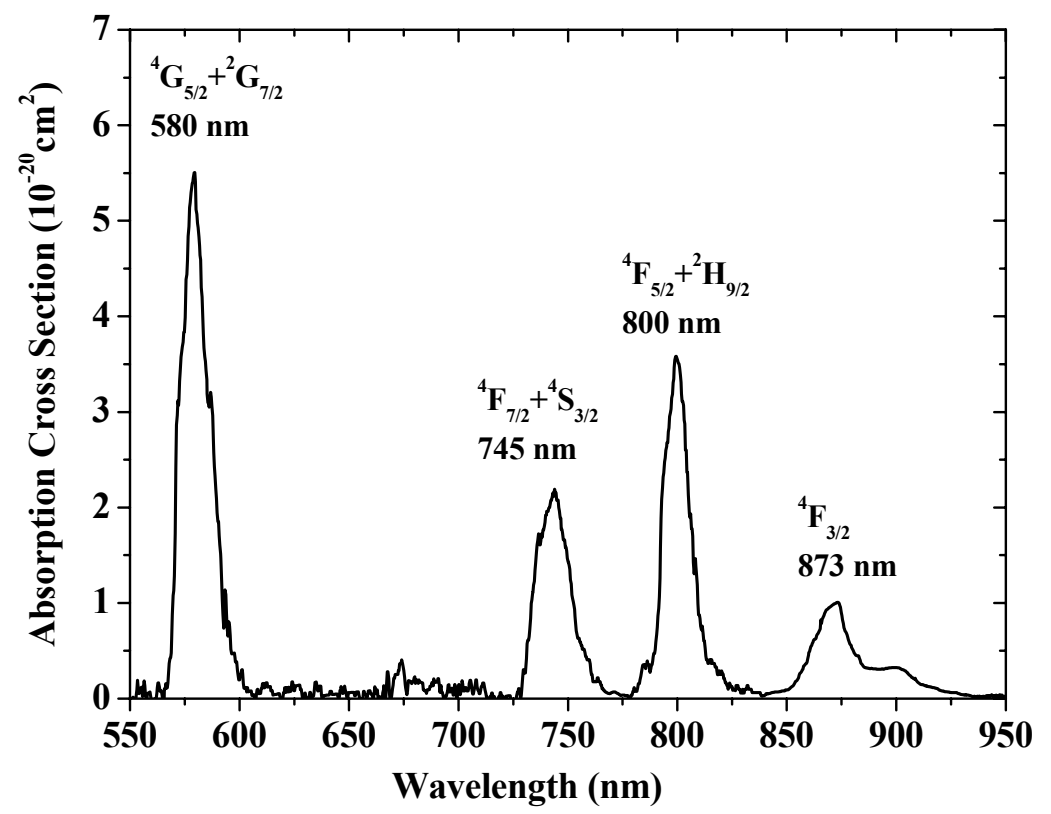

(b)

Fig. 3.19. Absorption (a) spectrum and (b) cross section of a $\mathrm{Nd}^{3+}$-complex-doped 6-FDA/UVR channel waveguide of $5 \times 5 \mu \mathrm{m}^{2}$. 
The single-wavelength losses at five different wavelengths and loss spectrum of $\mathrm{Al}_{2} \mathrm{O}_{3}: \mathrm{Nd}^{3+}$ slab waveguides were measured by the prism-coupling method and are given in Table 3.1 and Fig. 3.20.

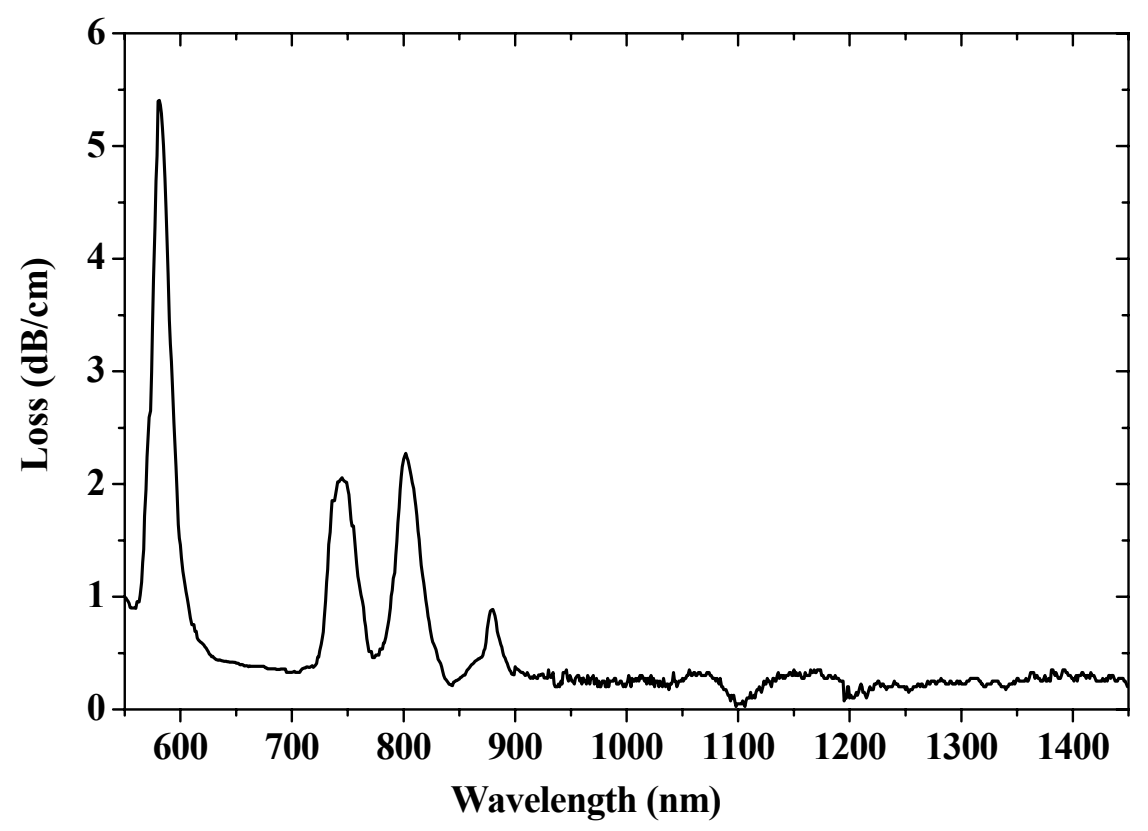

Fig. 3.20. Loss spectrum of an $\mathrm{Al}_{2} \mathrm{O}_{3}: \mathrm{Nd}^{3+}$ slab waveguide.

The loss spectrum was measured independent of polarization. The absorption spectrum and cross section of $\mathrm{Nd}^{3+}$ was obtained by subtracting the background propagation loss, which indicates four absorption peaks at $580 \mathrm{~nm}, 745$ $\mathrm{nm}, 800 \mathrm{~nm}$, and $870 \mathrm{~nm}$, corresponding the $\mathrm{Nd}^{3+}$ absorption transitions ${ }^{4} \mathrm{I}_{9 / 2} \rightarrow$ ${ }^{4} \mathrm{G}_{5 / 2}+{ }^{2} \mathrm{G}_{7 / 2},{ }^{4} \mathrm{I}_{9 / 2} \rightarrow{ }^{4} \mathrm{~F}_{7 / 2}+{ }^{4} \mathrm{~S}_{3 / 2},{ }^{4} \mathrm{I}_{9 / 2} \rightarrow{ }^{4} \mathrm{~F}_{5 / 2}+{ }^{2} \mathrm{H}_{9 / 2}$, and ${ }^{4} \mathrm{I}_{9 / 2} \rightarrow{ }^{4} \mathrm{~F}_{3 / 2}$, respectively (Fig. 3.21(a) and (b)).

The loss in $\mathrm{Al}_{2} \mathrm{O}_{3}$ waveguides without active dopants and with $\mathrm{Er}^{3+}$ dopants has been investigated in previous work [8-10] and indicated that very low extra propagation loss $(\sim 0.1 \mathrm{~dB} / \mathrm{cm})$ was introduced in channel waveguides by patterning. In this work, the single-wavelength loss of two $0.6 \times 2.0-\mu \mathrm{m}^{2}$ channel waveguides were investigated at $800 \mathrm{~nm}, 880 \mathrm{~nm}$, and $1064 \mathrm{~nm}$ and compared with the loss measured in slab waveguides (Table 3.1). Additional channel propagation losses of $0.6,0.3$ and $0.15 \mathrm{~dB} / \mathrm{cm}$ were measured in $\mathrm{Al}_{2} \mathrm{O}_{3}: \mathrm{Nd}^{3+}$ channel waveguides at 880,1064 and 1330 $\mathrm{nm}$, respectively. 


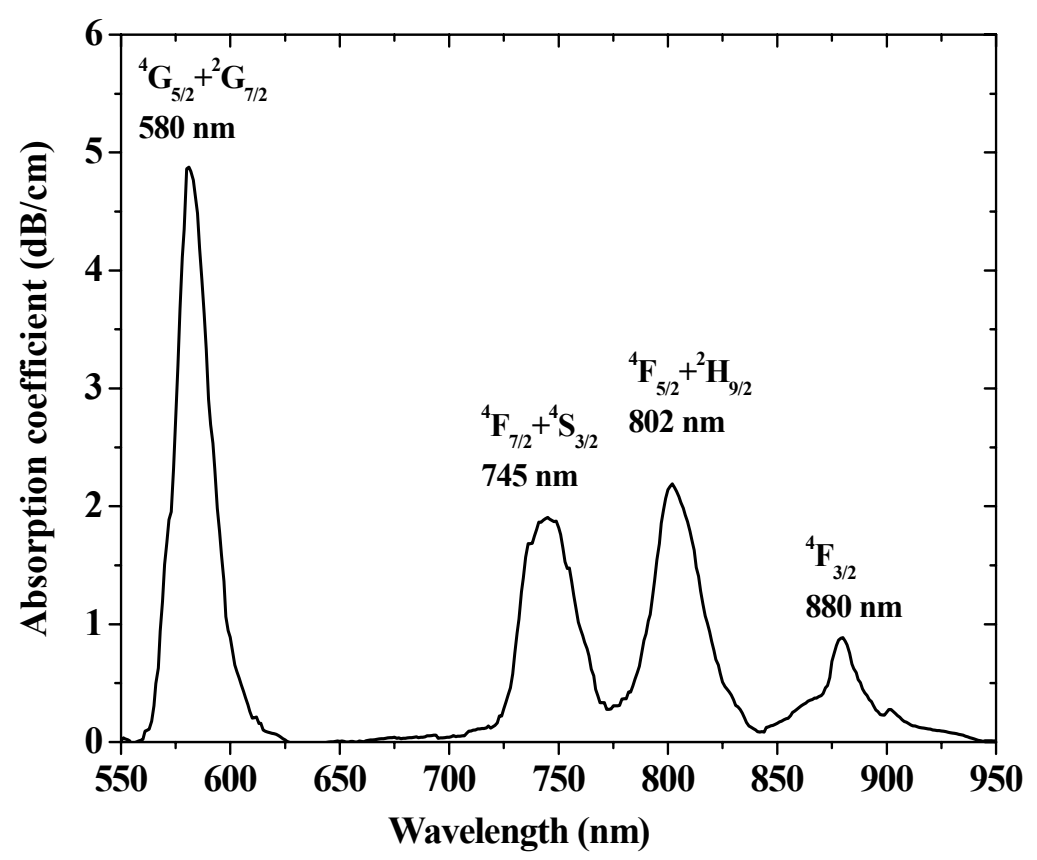

(a)

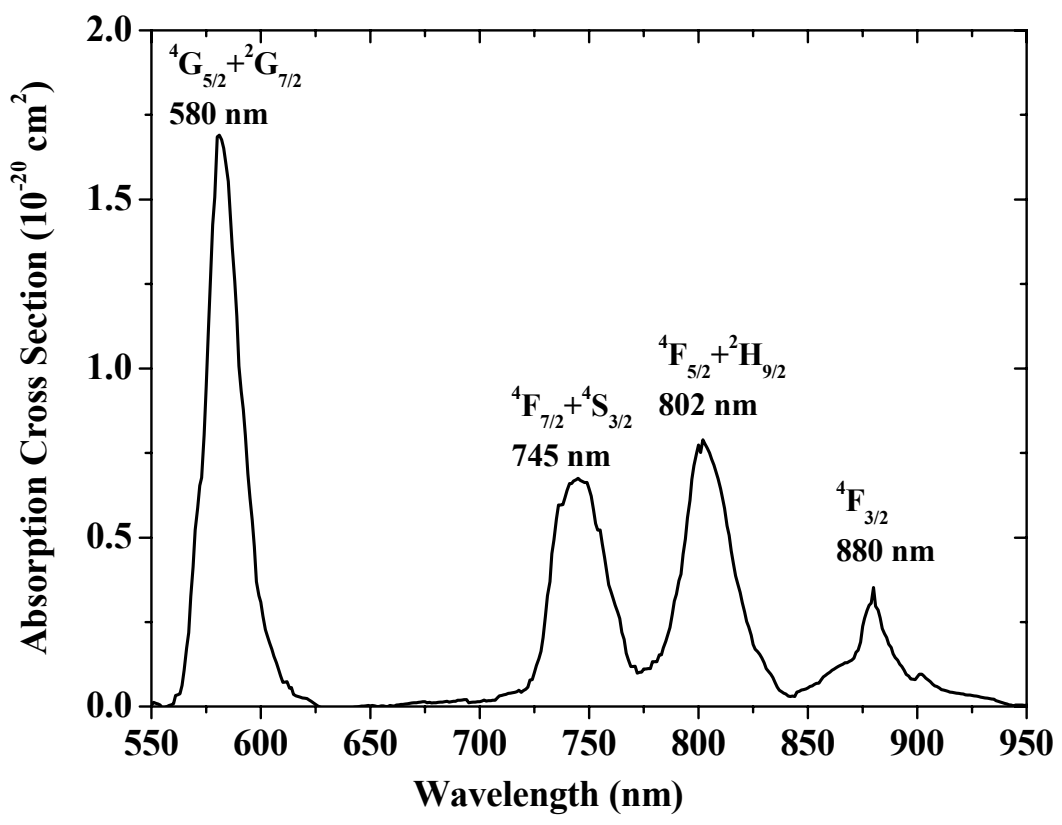

(b)

Fig. 3.21. Absorption (a) spectrum and (b) cross section of an $\mathrm{Al}_{2} \mathrm{O}_{3}: \mathrm{Nd}^{3+}$ slab waveguide.

In summary, 6-FDA/UVR has a broadband transmission and very low loss around $870 \mathrm{~nm}$ and $1064 \mathrm{~nm}$, which is important for optical amplification and lasing at these two wavelengths. However, this polymer material is not suitable for active waveguide devices at telecommunication wavelengths due to the dramatic increase of the optical loss at wavelengths longer than $1100 \mathrm{~nm}$. On the other hand, although $\mathrm{Al}_{2} \mathrm{O}_{3}$ has slightly higher loss than 6-FDA/UVR at wavelengths below $1100 \mathrm{~nm}$, it has very low loss around $1330 \mathrm{~nm}$, which is interesting for optical amplification at in the telecom O-band. 


\subsubsection{Judd-Ofelt Analysis}

The standard Judd-Ofelt analysis discussed in section 2.2.3 was applied for studying the optical transitions of $\mathrm{Nd}^{3+}$ ions in both the polymer and $\mathrm{Al}_{2} \mathrm{O}_{3}$ host material.

The Judd-Ofelt parameters $\Omega_{t}(t=2,4,6)$ were determined by the measured and calculated line strength $\left(S_{\text {meas }}\right.$ and $\left.S_{\text {calc }}\right)$ for a number of ground-state transitions.

In the previous Section, four absorption peaks of $\mathrm{Nd}^{3+}$ have been identified from the absorption spectra of $\mathrm{Nd}^{3+}$. The values of the doubly reduced matrix elements $U^{(t)}(t=2,4,6)$ at these four peak wavelengths of $\mathrm{Nd}^{3+}$, which are independent of the host material, are given in Table $3.2[82,83]$.

Table 3.2. Values of reduced matrix elements for the absorption transitions of $\mathrm{Nd}^{3+}$ at $300 \mathrm{~K}$.

\begin{tabular}{ccccc}
\hline \hline Transition (from ${ }^{4} \boldsymbol{I}_{\mathbf{9} / \mathbf{2}}$ ) & $\boldsymbol{\lambda}(\mathbf{n m})$ & {$\left[\boldsymbol{U}^{(\mathbf{2})}\right]^{\mathbf{2}}$} & {$\left[\boldsymbol{U}^{(\mathbf{4})}\right]^{\mathbf{2}}$} & {$\left[\boldsymbol{U}^{(\mathbf{6})}\right]^{\mathbf{2}}$} \\
\hline${ }^{4} G_{5 / 2}+{ }^{2} G_{7 / 2}$ & $\sim 580$ & 0.9710 & 0.5897 & 0.0663 \\
${ }^{4} F_{7 / 2}+{ }^{4} S_{3 / 2}$ & $\sim 745$ & 0.0010 & 0.0448 & 0.6582 \\
${ }^{4} F_{5 / 2}+{ }^{2} H_{9 / 2}$ & $\sim 800$ & 0.0100 & 0.2431 & 0.5148 \\
${ }^{4} F_{3 / 2}$ & $\sim 880$ & 0 & 0.2296 & 0.0563 \\
\hline
\end{tabular}

The measured and calculated line strength $S_{\text {meas }}$ and $S_{\text {calc }}$ of $\mathrm{Nd}^{3+}$ in 6FDA/UVR and $\mathrm{Al}_{2} \mathrm{O}_{3}$ are given in Table 3.3 (a) and (b) respectively. By a leastsquares fit of $S_{\text {meas }}$ and $S_{\text {calc }}$, the values of the Judd-Ofelt parameters $\Omega_{t}(t=2,4,6)$ of $\mathrm{Nd}^{3+}$ in each host material were obtained and are given in Table 3.4. With these parameters, the transition line strengths of the upper state transitions $\mathrm{S}_{\text {calc }}$, radiative decay rates $A_{J J}$, radiative lifetimes $\tau_{r a d}$, and branching ratios $B$ were obtained and are presented in Table 3.5.

Table 3.3. Measured and calculated line strengths of $\mathrm{Nd}^{3+}$ in (a) 6-FDA/UVR and (b) $\mathrm{Al}_{2} \mathrm{O}_{3}$ at $300 \mathrm{~K}$.

\begin{tabular}{|c|c|c|c|c|c|}
\hline $\begin{array}{l}\text { Transition } \\
\left(\text { from }^{4} I_{9 / 2}\right)\end{array}$ & $\begin{array}{c}\lambda \\
(\mathrm{nm})\end{array}$ & n & $\begin{array}{c}\left(\int \mathrm{a}(\lambda) \mathrm{d} \lambda\right) / N_{0} \\
\left(10^{-20} \mathbf{n m} \cdot \mathrm{cm}^{2}\right)\end{array}$ & $\begin{array}{c}S_{\text {meas }} \\
\left(10^{-20} \mathrm{~cm}^{2}\right)\end{array}$ & $\begin{array}{c}S_{\text {calc }} \\
\left(10^{-20} \mathrm{~cm}^{2}\right)\end{array}$ \\
\hline${ }^{4} G_{5 / 2}+{ }^{2} G_{7 / 2}$ & 580 & 1.5316 & 132.4814 & 17.3600 & 17.3700 \\
\hline${ }^{4} F_{7 / 2}+{ }^{4} S_{3 / 2}$ & 745 & 1.5239 & 58.0268 & 5.9600 & 6.2800 \\
\hline${ }^{4} F_{5 / 2}+{ }^{2} H_{9 / 2}$ & 800 & 1.5221 & 75.8987 & 7.2600 & 6.8100 \\
\hline${ }^{4} F_{3 / 2}$ & 873 & 1.5206 & 23.4766 & 2.0700 & 2.4800 \\
\hline
\end{tabular}

\begin{tabular}{|c|c|c|c|c|c|}
\hline $\begin{array}{l}\text { Transition } \\
\left(\text { from }^{4} I_{9 / 2}\right)\end{array}$ & $\begin{array}{c}\lambda \\
(\mathbf{n m})\end{array}$ & $\mathbf{n}$ & $\begin{array}{c}\left(\int \mathrm{a}(\lambda) \mathrm{d} \lambda\right) / N_{0} \\
\left(10^{-20} \mathbf{n m} \cdot \mathbf{c m}^{2}\right)\end{array}$ & $\begin{array}{c}S_{\text {meas }} \\
\left(10^{-20} \mathrm{~cm}^{2}\right)\end{array}$ & $\begin{array}{c}S_{\text {calc }} \\
\left(10^{-20} \mathrm{~cm}^{2}\right)\end{array}$ \\
\hline${ }^{4} G_{5 / 2}+{ }^{2} G_{7 / 2}$ & 580 & 1.655 & 43.4778 & 5.1363 & 5.1387 \\
\hline${ }^{4} F_{7 / 2}+{ }^{4} S_{3 / 2}$ & 745 & 1.661 & 26.8889 & 2.4809 & 2.6619 \\
\hline${ }^{4} F_{5 / 2}+{ }^{2} H_{9 / 2}$ & 802 & 1.660 & 31.6778 & 2.7239 & 2.4670 \\
\hline${ }^{4} F_{3 / 2}$ & 880 & 1.659 & 4.7889 & 0.3747 & 0.6051 \\
\hline
\end{tabular}


Table 3.4. Judd-Ofelt parameters of $\mathrm{Nd}^{3+}$ in 6-FDA/UVR and $\mathrm{Al}_{2} \mathrm{O}_{3}$.

\begin{tabular}{ccccc}
\hline \hline \multirow{2}{*}{ Material } & \multicolumn{2}{c}{ Judd-Ofelt parameters } & $\left(\mathbf{1 0} \mathbf{1 0}^{-20} \mathbf{c m}^{\mathbf{2}}\right)$ & RMS deviation \\
& $\Omega_{\mathbf{2}}$ & $\boldsymbol{\Omega}_{\mathbf{4}}$ & $\boldsymbol{\Omega}_{\mathbf{6}}$ & $\left.\mathbf{( 1 0}^{-20} \mathbf{c m}^{\mathbf{2}}\right)$ \\
\hline $\mathrm{Nd}^{3+}$-complex-doped polymer & 12.05 & 8.59 & 8.93 & 0.69 \\
$\mathrm{Al}_{2} \mathrm{O}_{3}: \mathrm{Nd}^{3+}$ & 4.01 & 1.67 & 3.92 & 0.39 \\
\hline
\end{tabular}

Table 3.5 Predicted fluorescence line strength $S_{\text {calc }}$, radiative decay rates $A_{J J}$, radiative lifetimes $\tau_{\text {rad }}$, and branching ratios $B$ of $\mathrm{Nd}^{3+}$ in (a) 6-FDA/UVR and (b) $\mathrm{Al}_{2} \mathrm{O}_{3}$ at $300 \mathrm{~K}$.

(a)

\begin{tabular}{cccccc}
\hline \hline \multirow{2}{*}{ Transition } & $\lambda$ & \multicolumn{4}{c}{$\mathbf{N d}^{3+}$-complex-doped 6-FDA/UVR } \\
& $\mathbf{( n m )}$ & $\boldsymbol{S}_{\text {calc }}\left(\mathbf{1 0}^{\mathbf{2 0}} \mathbf{c m}^{\mathbf{2}}\right)$ & $\boldsymbol{A}_{\boldsymbol{J} J^{\prime}}\left(\mathbf{m s}^{\mathbf{- 1}}\right)$ & $\boldsymbol{\tau}_{\text {rad }}(\boldsymbol{\mu s})$ & $\boldsymbol{B}$ \\
\hline${ }^{4} F_{3 / 2} \rightarrow{ }^{4} \mathrm{I}_{9 / 2}$ & $\sim 880$ & 2.4769 & 1.7901 & & 0.3906 \\
${ }^{4} F_{3 / 2} \rightarrow{ }^{4} \mathrm{I}_{11 / 2}$ & $\sim 1060$ & 4.8554 & 2.3320 & 218 & 0.5088 \\
${ }^{4} F_{3 / 2} \rightarrow{ }^{4} \mathrm{I}_{13 / 2}$ & $\sim 1330$ & 1.8934 & 0.4388 & & 0.0957 \\
${ }^{4} F_{3 / 2} \rightarrow{ }^{4} \mathrm{I}_{15 / 2}$ & $\sim 1800$ & 0.2501 & 0.0224 & & 0.0049 \\
\hline
\end{tabular}

(b)

\begin{tabular}{|c|c|c|c|c|c|}
\hline \multirow{2}{*}{ Transition } & \multirow{2}{*}{$\begin{array}{c}\lambda \\
(\mathbf{n m})\end{array}$} & \multicolumn{4}{|c|}{ 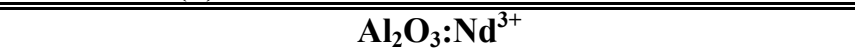 } \\
\hline & & $S_{\text {calc }}\left(10^{-20} \mathrm{~cm}^{2}\right)$ & $A_{J J^{\prime}}\left(\mathrm{ms}^{-1}\right)$ & $\tau_{\text {rad }}(\mu \mathbf{s})$ & $B$ \\
\hline${ }^{4} F_{3 / 2} \rightarrow{ }^{4} \mathrm{I}_{9 / 2}$ & $\sim 880$ & 0.6046 & 0.6761 & \multirow{4}{*}{474} & 0.3206 \\
\hline${ }^{4} F_{3 / 2} \rightarrow{ }^{4} \mathrm{I}_{11 / 2}$ & $\sim 1060$ & 1.8347 & 1.1647 & & 0.5522 \\
\hline${ }^{4} F_{3 / 2} \rightarrow{ }^{4} \mathrm{I}_{13 / 2}$ & $\sim 1330$ & 0.8319 & 0.2551 & & 0.1210 \\
\hline${ }^{4} F_{3 / 2} \rightarrow{ }^{4} \mathrm{I}_{15 / 2}$ & $\sim 1800$ & 0.1099 & 0.0131 & & 0.0062 \\
\hline
\end{tabular}

According to Reisfeld and Jørgensen [90], the $\Omega_{2}$ parameter is a measure of the covalence degree related to the established rare earth ion and ligand bonds, and gives information about the asymmetry of the local environment of the $\mathrm{Nd}^{3+}$ ion site. Symmetric fields of the $\mathrm{Nd}^{3+}$ ions lead to a decrease of the $\Omega_{2}$ values, because of the domination of magnetic dipole transitions, along with a decrease in the electric dipole transitions. The weaker the $\Omega_{2}$ value, the more centrosymmetric the ion site is and the more ionic its chemical bonds with the ligands are.

Comparison of the values of the Judd-Ofelt parameters of $\mathrm{Nd}^{3+}$ in 6FDA/UVR and $\mathrm{Al}_{2} \mathrm{O}_{3}$ in our work with other hosts as shown in Table 3.6, our $\mathrm{Nd}(\mathrm{TTA})_{3}$ phen-doped $6 \mathrm{FDA} / \mathrm{UVR}$ has a large $\Omega_{2}$ which agrees with the nonsymmetry of the $\mathrm{Nd}^{3+}$ local environment in the complex. On the other hand, the $\Omega_{6}$ parameter is related to the stiffness of the host matrix and shows a different type of variation related to the rigidity of the medium. Generally, it increases in the order (crystalline mixed oxides $<$ glasses $<$ viscous solutions $<$ hydrated ions $<$ halide vapors $<$ complexes of organic ligands) [90], which explains the high value of $\Omega_{6}$ in our polymer host.

Another point to be mentioned is that in the case of $\mathrm{Nd}^{3+}$ ions, the matrix elements $\left\langle{ }^{4} \mathrm{~F}_{3 / 2}\left\|U^{2}\right\|{ }^{4} \mathrm{I}_{\mathrm{J}}>\right.$ are all zero. It means that the near infrared emissions are uniquely governed by the $\Omega_{4}$ and $\Omega_{6}$. In this respect, Jacobs and Weber introduced a spectroscopic quality parameter $X$, equal to the ratio $\Omega_{4} / \Omega_{6}$. It has been observed that $X$ is usually in the range from 0.2 to 1.5 in a wide range of $\mathrm{Nd}^{3+}$ doped hosts [116]. In 
the case of $\mathrm{X} \leq 1$, the efficiency of the ${ }^{4} \mathrm{~F}_{3 / 2} \rightarrow{ }^{4} \mathrm{I}_{11 / 2}$ transition is enhanced, while the larger the value $X$, the more intense the transition ${ }^{4} \mathrm{~F}_{3 / 2} \rightarrow{ }^{4} \mathrm{I}_{9 / 2}$ is. According to this, the ${ }^{4} \mathrm{~F}_{3 / 2} \rightarrow{ }^{4} \mathrm{I}_{11 / 2}$ transition of $\mathrm{Nd}^{3+}$ in $\mathrm{Al}_{2} \mathrm{O}_{3}$ is favored compared to the ${ }^{4} \mathrm{~F}_{3 / 2} \rightarrow{ }^{4} \mathrm{I}_{9 / 2}$ transition due to the low $X$ value.

Table 3.6. Judd-Ofelt parameters and radiative lifetime of $\mathrm{Nd}^{3+}$ in different hosts.

\begin{tabular}{|c|c|c|c|c|c|c|}
\hline \multirow{2}{*}{ Host } & \multicolumn{4}{|c|}{ Judd-Ofelt parameters $\left(10^{-20} \mathrm{~cm}^{2}\right)$} & \multirow{2}{*}{$\begin{array}{l}\tau_{\text {rad }} \\
(\mu \mathrm{s})\end{array}$} & \multirow[t]{2}{*}{ Ref. } \\
\hline & $\Omega_{2}$ & $\Omega_{4}$ & $\Omega_{6}$ & $X=\Omega_{4} / \Omega_{6}$ & & \\
\hline 6-FDA/UVR & 12.10 & 8.59 & 8.93 & 0.96 & 218 & This work \\
\hline $\mathrm{Al}_{2} \mathrm{O}_{3}$ & 4.01 & 1.67 & 3.92 & 0.43 & 474 & This work \\
\hline phosphate glass & 3.28 & 3.54 & 4.67 & 0.76 & 430 & [48] \\
\hline YAG & 0.20 & 2.70 & 5.00 & 0.54 & 259 & [91] \\
\hline NGAB & 1.89 & 2.55 & 4.95 & 0.52 & 293 & [92] \\
\hline sulphide glass & 8.20 & 5.30 & 3.80 & 1.39 & 109 & [93] \\
\hline ZBLAN & 2.37 & 3.77 & 4.85 & 0.78 & 444 & [94] \\
\hline $\mathrm{KLa}\left(\mathrm{WO}_{4}\right)_{2}$ & 3.09 & 1.04 & 1.04 & 1.00 & 685 & [113] \\
\hline lead silicate glass (SNPZ) & 3.66 & 5.53 & 2.73 & 2.03 & 313 & [114] \\
\hline PF plastic solution & 10.60 & 6.51 & 4.72 & 1.38 & 548 & [115] \\
\hline PMMA-d $_{8}$ & 9.45 & 2.70 & 5.27 & 0.51 & 187 & [115] \\
\hline $\mathrm{ZBAN}^{51}$ & 3.10 & 3.70 & 5.70 & 0.65 & 419 & [115] \\
\hline
\end{tabular}

\subsubsection{Determination of $\mathrm{Nd}^{3+}$ Concentration in Host Materials}

The value of the $\mathrm{Nd}^{3+}$ concentration in host materials is necessary for the characterization of $\mathrm{Nd}^{3+}$-doped systems.

The concentration $N_{0}$ of $\mathrm{Nd}^{3+}$ ions in polymer materials can be easily determined by the following equation:

$$
N_{0}=\frac{x \cdot 6.023 \cdot 10^{23} / M}{V_{\text {solution }}},
$$

where $x$ is the weight of the $\mathrm{Nd}^{3+}$-complex added into the solution, $M$ is the molecular weight of the $\mathrm{Nd}^{3+}$-complex, $\mathrm{Nd}(\mathrm{TTA})_{3}$ phen, and $V_{\text {solution }}$ is the volume of the solutes (6-FDA and UVR) in the polymer solution.

As mentioned in Section 3.3.1, the $\mathrm{Nd}^{3+}$ concentration in $\mathrm{Al}_{2} \mathrm{O}_{3}$ can be measured by RBS. The open dots in Fig. 3.22 are the $\mathrm{Nd}^{3+}$ concentration in $\mathrm{Al}_{2} \mathrm{O}_{3}: \mathrm{Nd}^{3+}$ films determined by RBS versus measured absorption peaks at $880 \mathrm{~nm}$. According to Eq. 2.18, the wavelength-dependent absorption intensity of $\mathrm{Nd}^{3+}$ at one single wavelength is directly proportional to the ion concentration, and a linear fit of the concentration value measured by RBS through origin is given in Fig. 3.22.

Such a linear fitting line can be used as reference to determine the concentration of $\mathrm{Nd}^{3+}$ in other $\mathrm{Al}_{2} \mathrm{O}_{3}: \mathrm{Nd}^{3+}$ samples by fitting the measured absorption peak of each sample at $880 \mathrm{~nm}$ on it. The $\mathrm{Nd}^{3+}$ concentrations given by closed dots in 
Fig. 3.22 were estimated by this way. Therefore, once the $\mathrm{Nd}^{3+}$ concentration of a group of $\mathrm{Al}_{2} \mathrm{O}_{3}: \mathrm{Nd}^{3+}$ samples have been measured by $\mathrm{RBS}$, the $\mathrm{Nd}^{3+}$ concentration of the rest of $\mathrm{Al}_{2} \mathrm{O}_{3}: \mathrm{Nd}^{3+}$ samples can be determined directly via absorption measurement. The accuracy of this approach is mainly dependent on the accuracy of the RBS measurement and absorption measurement.

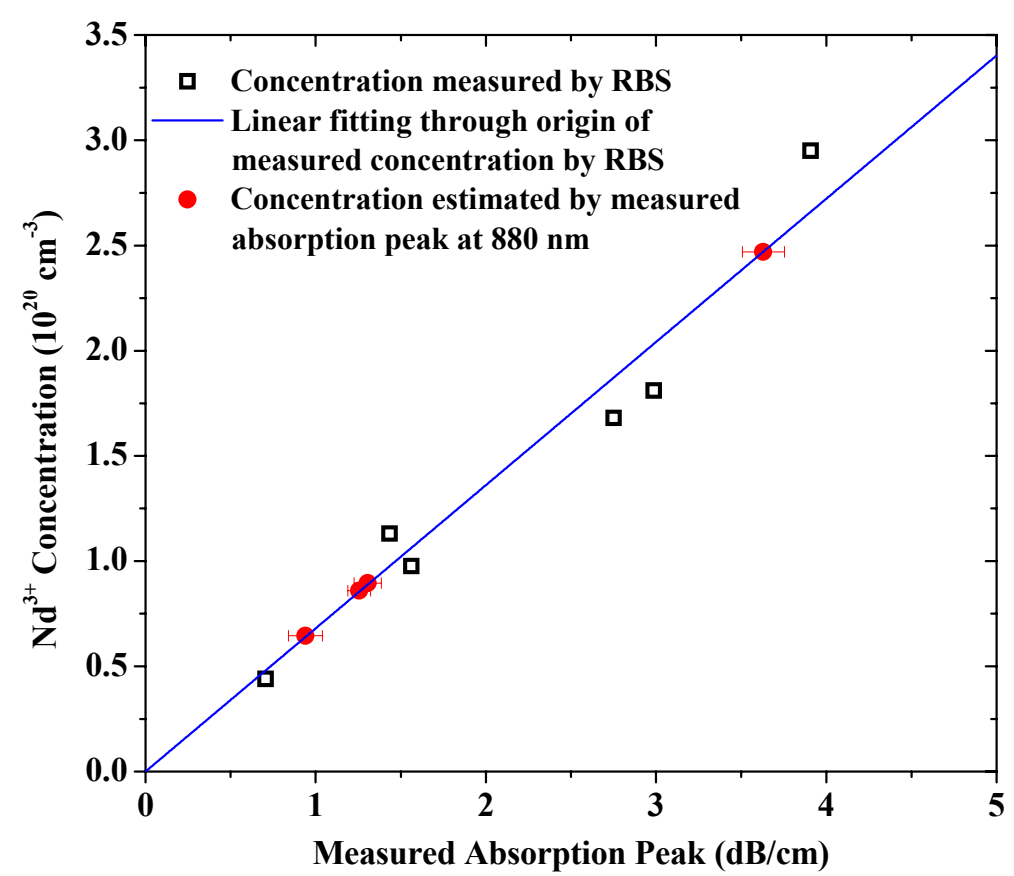

Fig. 3.22. $\mathrm{Nd}^{3+}$ concentration of $\mathrm{Nd}^{3+}$ in $\mathrm{Al}_{2} \mathrm{O}_{3}$ layers determined by $\mathrm{RBS}$ or absorption measurements versus absorption peak values at $880 \mathrm{~nm}$.

\subsection{Luminescence and Lifetime Measurements}

\subsubsection{Luminescence Spectra}

The photoluminescence spectrum is the first step to study the luminescence properties of the active ions in a host material. Figure 3.23 illustrates the photoluminescence measurement in a channel waveguide. Photoluminescence spectra of $\mathrm{Nd}^{3+}$ in the 6FDA/epoxy and $\mathrm{Al}_{2} \mathrm{O}_{3}$ hosts were investigated by exciting the active-ion-doped waveguides using a Ti:Sapphire laser at $800 \mathrm{~nm}$. The luminescence light was collected from the top of the waveguides by a large-core liquid-core guiding fiber, and luminescence peaks were detected by a spectrometer (iHR550, Horiba, Jobin Yvon).

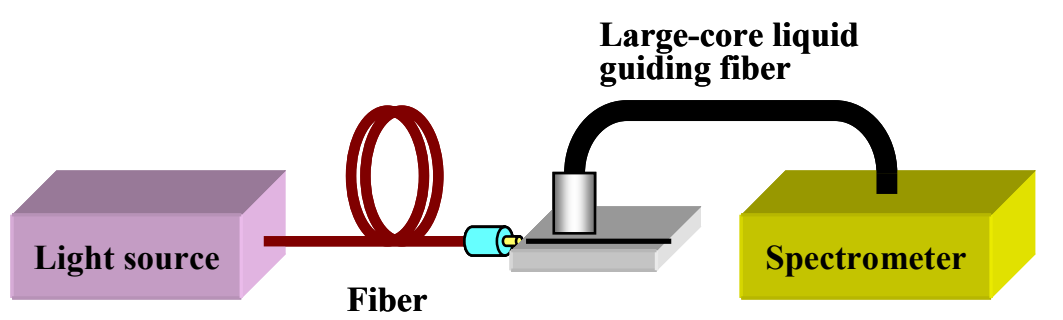

Fig. 3.23. Schematic picture of a photoluminescence measurement setup in a channel waveguide. 
An InGaAs detector was used to measure the broadband spectrum around $830-1450 \mathrm{~nm}$. However, the response curve of the InGaAs detector is very low and not flat around $800-950 \mathrm{~nm}$. Furthermore, the sensitivity of the grating used for measuring the broadband spectrum is also low around this wavelength range. Therefore, the luminescence spectrum around 830-950 $\mathrm{nm}$ was also measured using a photomultiplier tube (PMT) detector. All the measured luminescence spectra were corrected with respect to the response curve of the detectors.

Figure 3.24(a) shows a broadband luminescence spectrum of a $\mathrm{Nd}^{3+}$ complex-doped 6-FDA/UVR channel waveguide. Three luminescence bands near 873 $\mathrm{nm}, 1056 \mathrm{~nm}$, and $1328 \mathrm{~nm}$ can be identified, corresponding to the transitions between the metastable state ${ }^{4} \mathrm{~F}_{3 / 2}$ and the lower lying ${ }^{4} \mathrm{I}_{9 / 2},{ }^{4} \mathrm{I}_{11 / 2}$, and ${ }^{4} \mathrm{I}_{13 / 2}$ states of the $\mathrm{Nd}^{3+}$ ion, respectively. The spectrum at 1000-1500 nm was measured by an InGaAs detector, while the first part of the spectrum at $800-1000 \mathrm{~nm}$, which includes the ground-state transition ${ }^{4} \mathrm{~F}_{3 / 2} \rightarrow{ }^{4} \mathrm{I}_{9 / 2}$ of $\mathrm{Nd}^{3+}$, was measured by a PMT. The spectrum was corrected with respect to the response curve of the two detectors, and adjusted to the normalized intensity. Using the Füchtbauer-Ladenburg theory (see Section 2.2.3), the emission cross section of $\mathrm{Nd}^{3+}$ was calculated from the measured photoluminescence spectrum with Eq. 2.20 (see Fig. 3.24(b)).

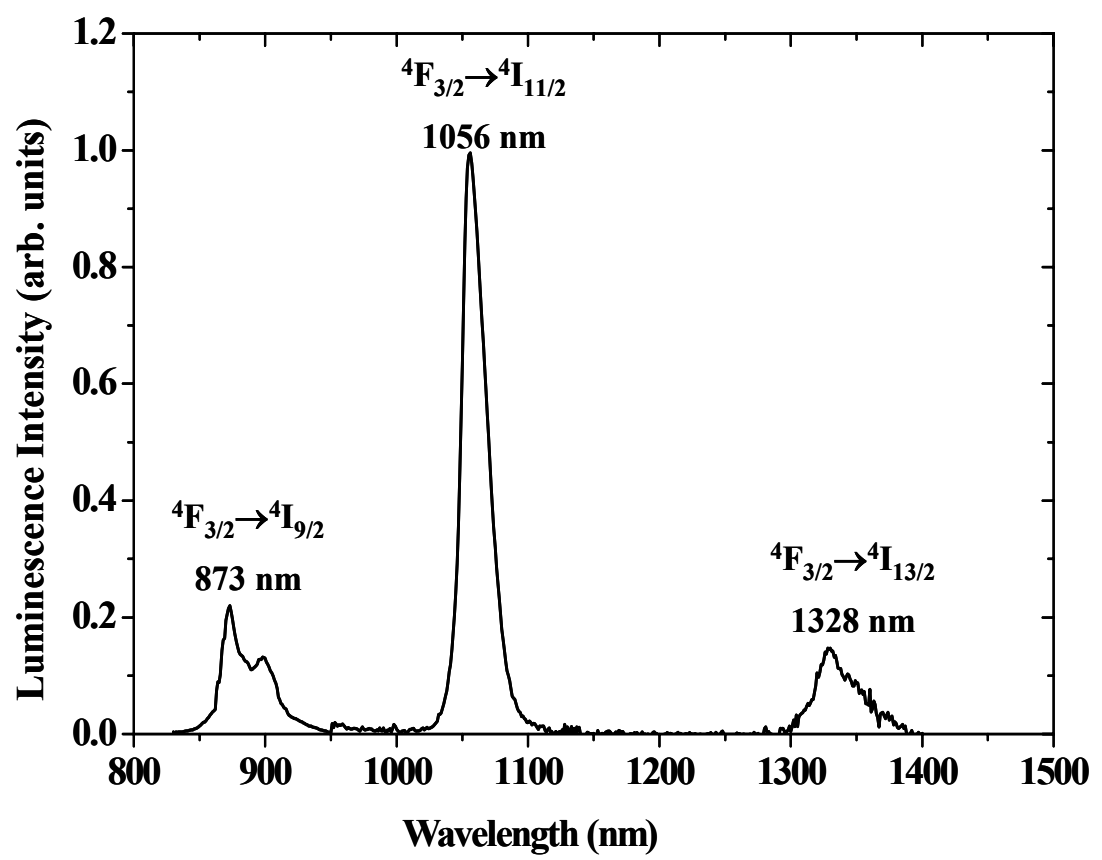

(a) 


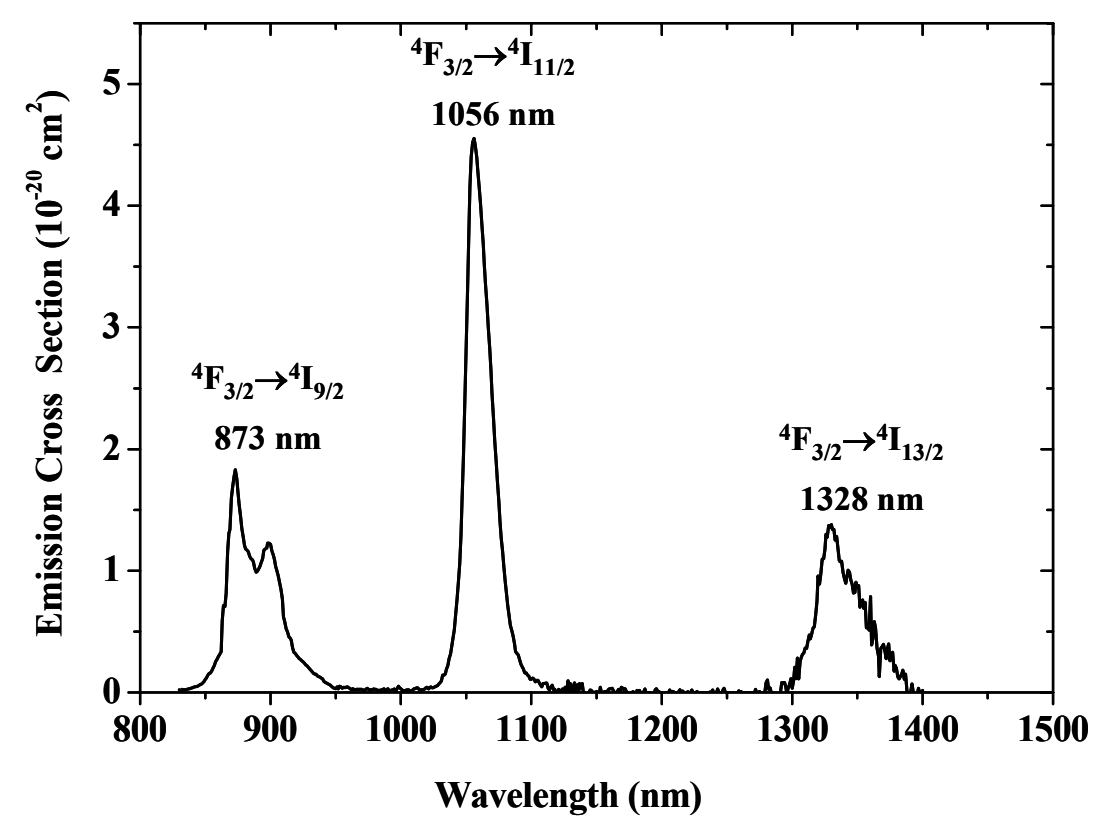

(b)

Fig. 3.24. Photoluminescence (a) spectrum and (b) cross section of a $\mathrm{Nd}^{3+}$-complex-doped 6FDA/UVR channel waveguide.

The luminescence spectra indicate that $\mathrm{Nd}^{3+}$ in 6-FDA/UVR and $\mathrm{Al}_{2} \mathrm{O}_{3}$ is optically active at all three transitions, ${ }^{4} F_{3 / 2} \rightarrow{ }^{4} \mathrm{I}_{9 / 2},{ }^{4} F_{3 / 2} \rightarrow{ }^{4} \mathrm{I}_{11 / 2}$, and ${ }^{4} F_{3 / 2} \rightarrow{ }^{4} \mathrm{I}_{13 / 2}$. The full width at half maximum of the three transitions are broader and less structured than that of $\mathrm{Nd}^{3+}$ luminescence in crystals [15], thus providing a large gain bandwidth in an optical amplifier.

The luminescence spectrum and cross section of an $\mathrm{Al}_{2} \mathrm{O}_{3}: \mathrm{Nd}^{3+}$ channel waveguide were obtained in the same way and are given in Fig. 3.25. Three luminescence peaks at $880 \mathrm{~nm}, 1065 \mathrm{~nm}$, and $1340 \mathrm{~nm}$ were recorded, which have a slight shift of 7-12 nm to longer wavelengths compared with those peaks in the $\mathrm{Nd}^{3+}$ complex-doped 6-FDA/UVR. 


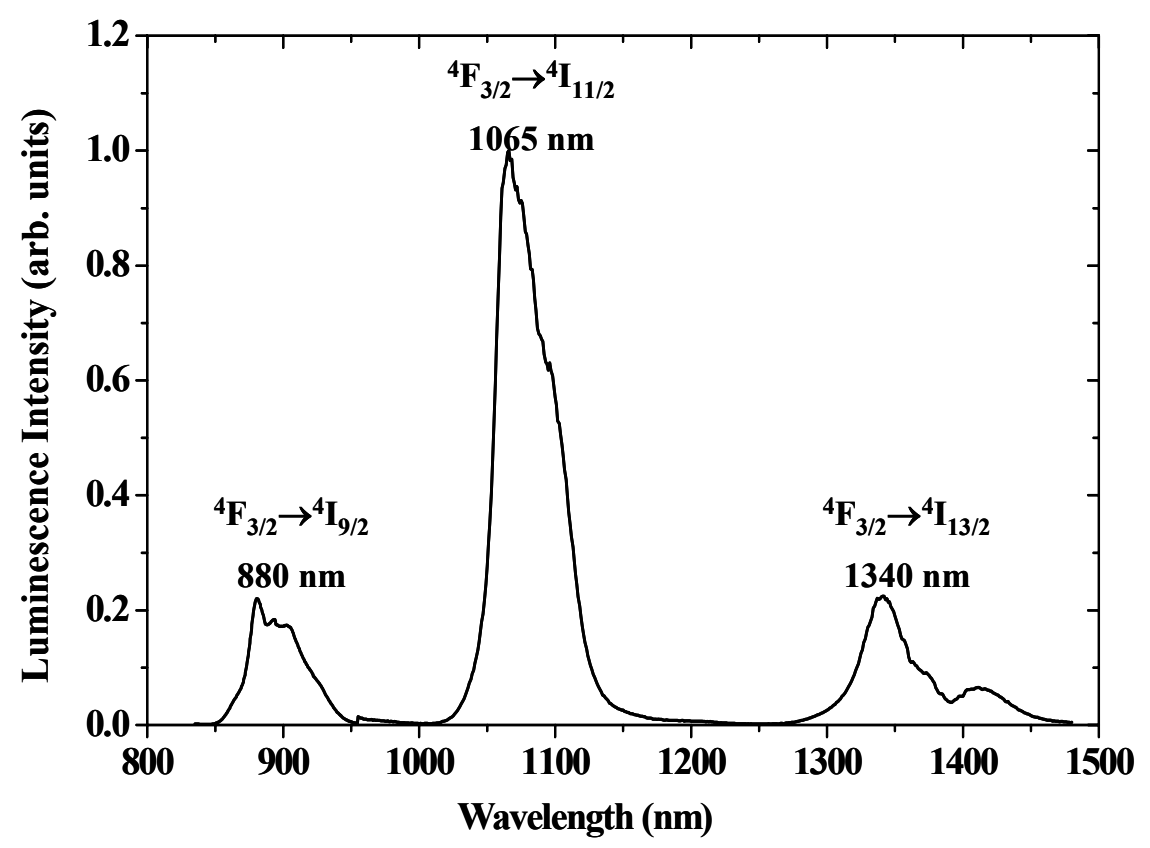

(a)

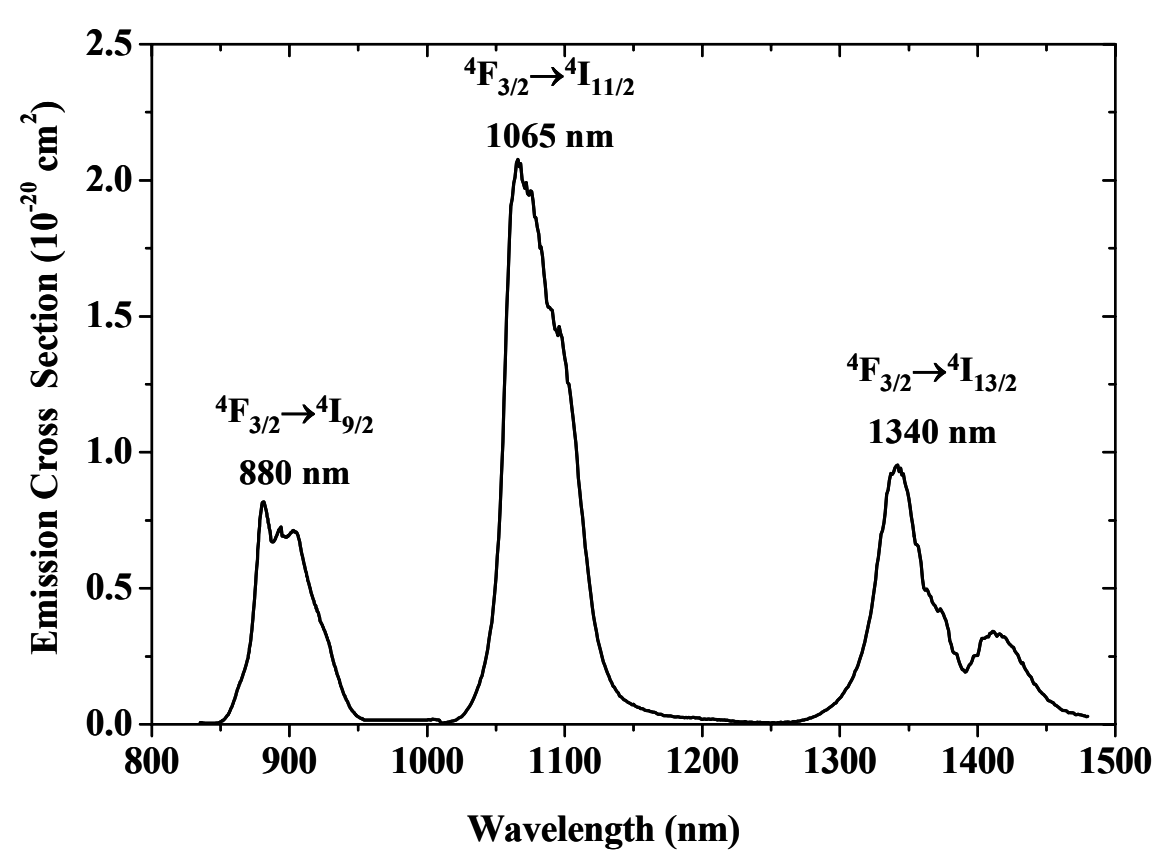

(b)

Fig. 3.25. Photoluminescence (a) spectrum and (b) cross section of an $\mathrm{Al}_{2} \mathrm{O}_{3:} \mathrm{Nd}^{3+}$ channel waveguide.

\subsubsection{Luminescence Lifetime}

The setup of the luminescence lifetime measurement is depicted in Fig. 3.26. Luminescence lifetime measurements of the $\mathrm{Nd}^{3+}$ ions in the 6-FDA/UVR and $\mathrm{Al}_{2} \mathrm{O}_{3}$ hosts were performed using a laser diode emitting at $800 \mathrm{~nm}$ as the excitation source. The laser diode was modulated by an external square-pulse generator and delivered pulses of $4 \mathrm{~ms}$ duration, allowing the populations of the $\mathrm{Nd}^{3+}$ system to reach a steady state before the pump was switched off. The pulse had a cut-off time of below $5 \mu \mathrm{s}$, 
which was much shorter of the luminescence lifetime of $\mathrm{Nd}^{3+}$. The luminescence light was collected from the top surface of the waveguide by a large-core liquid fiber, and diffracted by a monochromator tuned to the peak wavelengths $(880 / 1060 / 1330 \mathrm{~nm})$. The selected luminescence light was detected by an InGaAs photodiode. The signal was amplified and then acquired with a digital oscilloscope.

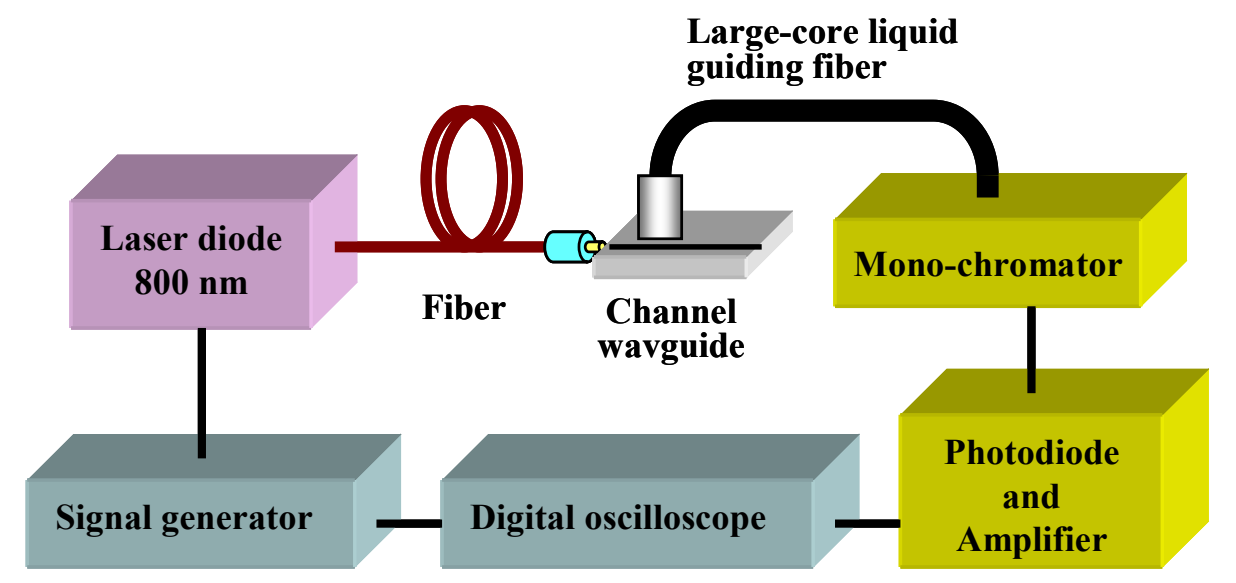

Fig. 3.26. Schematic of a setup for measuring the luminescence lifetime in channel waveguides.

Figure 3.27 show the luminescent decay of $\mathrm{Nd}^{3+}$ measured in 6-FDA/UVR at $1060 \mathrm{~nm}$ of $\mathrm{Nd}^{3+}$ concentration of $1.03 \times 10^{20} \mathrm{~cm}^{-3}$.

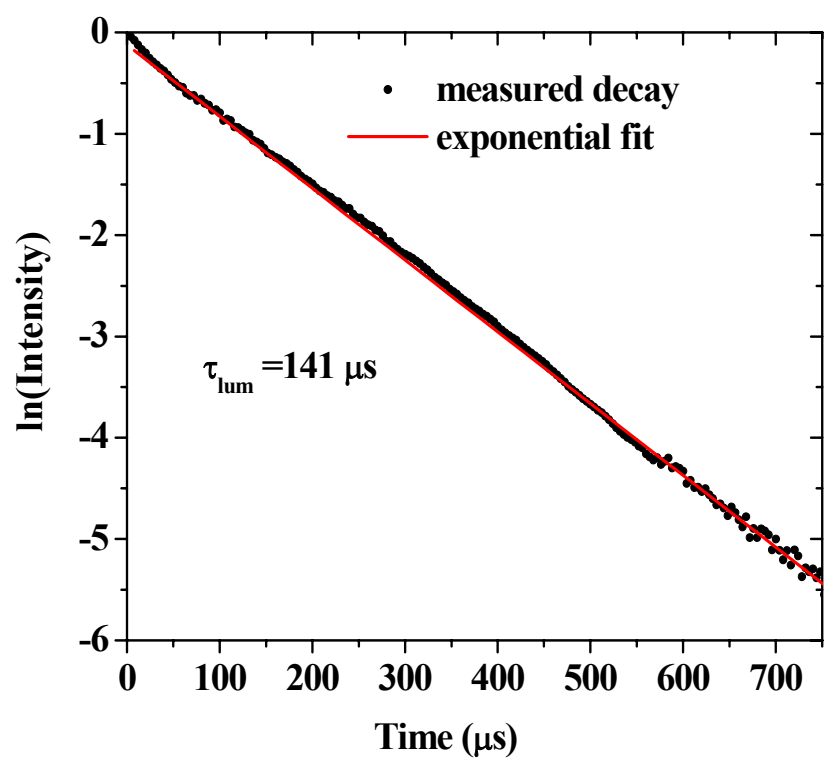

Fig. 3.27. Luminescence decay of $\mathrm{Nd}^{3+}$ in a 6-FDA/UVR channel waveguide of $\mathrm{Nd}^{3+}$ concentration of $1.03 \times 10^{20} \mathrm{~cm}^{-3}$.

Except for a faster decay occurring directly after switching off the pump excitation (the first $20 \mu \mathrm{s}$ ) in Fig. 3.27, which is attributed to ETU between neighboring $\mathrm{Nd}$ ions in their ${ }^{4} \mathrm{~F}_{3 / 2}$ excited levels [54], an exponential decay was observed. A luminescence lifetime of $141 \mu \mathrm{s}$ at $1056 \mathrm{~nm}$ was derived from the exponential part of the decay curve, which was independent of the excitation intensity. 
This long lifetime demonstrates that luminescence quenching effects have been largely diminished by the choice of complex and surrounding polymer materials. Furthermore, encapsulating the Nd ions may well contribute to diminishing the effect of ETU by ensuring a rather large distance between nearest-neighbor Nd ions. The long, unquenched excited-state lifetime of $\mathrm{Nd}$ ions in this specific environment sets the stage for successful gain demonstration as well as continuous-wave polymer laser experiments.

Much stronger intensity of the luminescence light can be collected from $\mathrm{Al}_{2} \mathrm{O}_{3}: \mathrm{Nd}^{3+}$ samples without top-cladding layer than that measured in polymer samples of top-cladding layer. Luminescence decay of $\mathrm{Nd}^{3+}$ in $\mathrm{Al}_{2} \mathrm{O}_{3}$ channel waveguides was measured with various ion concentrations and is given in Fig. 3.28(a).

In the decay curve (1) of the lowest concentration, except for a faster decay occurring directly after switching off the pump excitation (see the first $\sim 50 \mu \mathrm{s}$ in Fig. 3.28(a), which is attributed to ETU between neighboring $\mathrm{Nd}$ ions in their ${ }^{4} \mathrm{~F}_{3 / 2}$ excited levels [88, 89], an exponential decay was observed. A luminescence lifetime of 325 $\mu$ s was derived from the exponential part of the decay curve at $1065 \mathrm{~nm}$, which was independent of the excitation intensity. It is of the same order of magnitude as the radiative lifetime of $474 \mu$ s calculated by Judd-Ofelt analysis (Table 3.4). However, the decay curves (2)-(4) at higher $\mathrm{Nd}^{3+}$ concentrations cannot be fitted with an exponential curve mainly due to the effects of ETU.

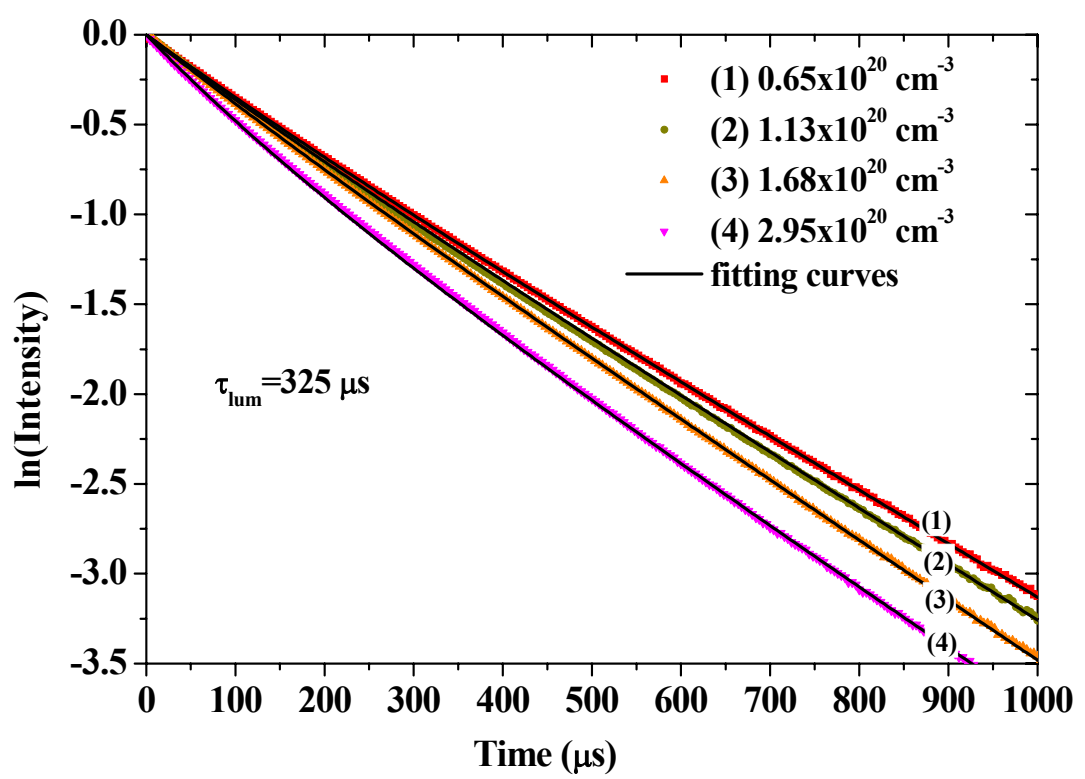

(a) 


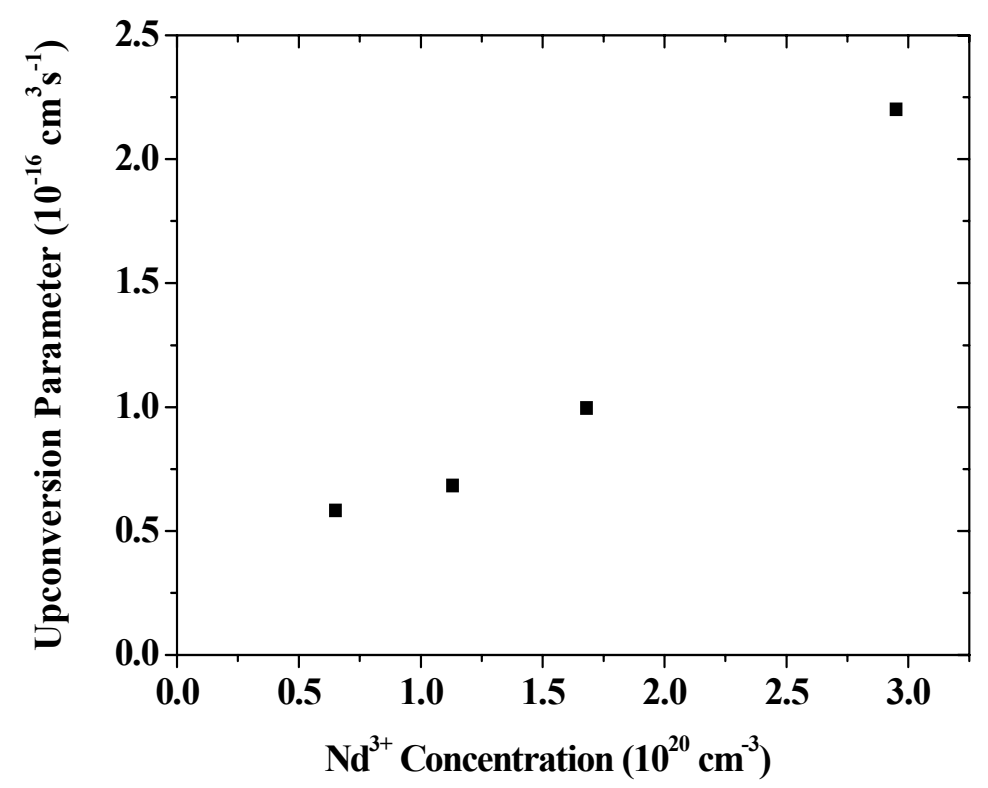

(b)

Fig. 3.28. (a) Luminescence decays of $\mathrm{Nd}^{3+}$ in $\mathrm{Al}_{2} \mathrm{O}_{3}$ channel waveguides; (b) ETU parameter of $\mathrm{Nd}^{3+}$ in $\mathrm{Al}_{2} \mathrm{O}_{3}$ derived from luminescence decay curves.

As discussed in section 2.3.1, 2.3.3 and 2.3.5, three ETU processes shown in the diagram of energy level of $\mathrm{Nd}^{3+}$ (Fig. 2.3) originating in the metastable ${ }^{4} \mathrm{~F}_{3 / 2}$ level of the $\mathrm{Nd}^{3+}$ system were taken into account and expressed by a single macroscopic parameter $W_{\mathrm{ETU}}$ in the simulation, as these processes lead to similar results concerning the population dynamics in the $\mathrm{Nd}^{3+}$ system. A non-exponential model based on the Bernoulli equation was applied to determine the ETU parameter. By setting the pump and signal terms to zero and solving Eq. (2.41) or (2.46) time-dependently, the Bernoulli equation is derived [117]:

$$
N(t)=\frac{N_{0} \exp (-t / \tau)}{1+W_{E T U} N_{0} \tau[1-\exp (-t / \tau)]}
$$

The intrinsic lifetime $\tau_{4}$ equals the luminescence lifetime obtained from the exponential decay at very low dopant concentration; it remains constant at all dopant concentrations. In this approach, we assumed that a sea of excitations smeared out over the excitation volume by infinitely fast energy migration within the ${ }^{4} \mathrm{~F}_{3 / 2}$ level. Therefore it is a macroscopic treatment, and valid only in the "kinetic limit" of the migration accelerated regime of ETU. The ETU parameter was determined by fitting Eq. 3.10 to the measured luminescence decay curves. The fitted curves show very good agreement with the measured decay curves at $1064 \mathrm{~nm}$ (see Fig. 3.28(a)), which is an indication that this simplified model is valid for ETU in the $\mathrm{Al}_{2} \mathrm{O}_{3}: \mathrm{Nd}^{3+}$ system. With this method, values of the ETU parameter of $0.58,0.68,1.00$, and $2.20 \times 10^{-16}$ $\mathrm{cm}^{3} \mathrm{~s}^{-1}$ were determined at $\mathrm{Nd}^{3+}$ concentrations of $0.65,1.13,1.68$, and $2.95 \times 10^{20} \mathrm{~cm}^{-3}$, respectively (Fig. 3.28(b)).

With this metod, ETU parameters of $0.58,0.68,1.00$, and $2.20 \times 10^{-16} \mathrm{~cm}^{3} \mathrm{~s}^{-1}$ were determined at $\mathrm{Nd}^{3+}$ concentrations of $0.65,1.13,1.68$, and $2.95 \times 10^{20} \mathrm{~cm}^{-3}$, respectively (see Fig. 3.28(b)). The main fitting error of the ETU parameters in this 
approach could be the deviation of the starting fitting point, due to the fluctuation of the excitation laser output. More details of ETU parameter of $\mathrm{Nd}^{3+}$ ions will be discussed in the next chapter.

For direct excitation, the quantum efficiency, $\eta_{q}$, is defined as

$$
\eta_{q}=\frac{\text { emitted light intensity }}{\text { absorbed pump intensity }}
$$

The maximum fluorescence quantum yield is 1.0 (100\%), when every photon absorbed results in a photon emitted. In the most of the case, the quantum efficiency is lower than 1.0 due to the nonradiative quenching. High quantum efficiency is required for efficient amplification and lasing. The quantum efficiency can be calculated from measured luminescence lifetime $\tau_{l u m}$ and calculated radiative lifetime $\tau_{r a d}$ using JuddOfelt theory by the equation:

$$
\eta_{q}=\tau_{\text {lum }} / \tau_{\text {rad }}
$$

The radiative lifetime, luminescence lifetime and quantum efficiency of $\mathrm{Nd}^{3+}$ in different host materials are given in Table 3.7. $\mathrm{Nd}^{3+}$ exhibits a shorter lifetime in 6FDA/UVR than in other inorganic hosts mainly due to the luminescent quenching in polymers, while the lifetime of $\mathrm{Nd}^{3+}$ in $\mathrm{Al}_{2} \mathrm{O}_{3}$ is longer indicating less quenching in this host. However, the accuracy of the calculated quantum efficiency by this approach is strongly dependent on the accuracy radiative lifetime derived by the JuddOfelt theory. Typically an error of a factor of 2 is associated with this theory, which explains the quantum efficiency of $\mathrm{Nd}^{3+}$ larger than unity in sulphide glass and ZBLAN in Table 3.7.

Table 3.7. Radiative lifetime $\tau_{\text {rad }}$, luminescence lifetime $\tau_{l u m}$, and quantum efficiency $\eta_{q}$ of $\mathrm{Nd}^{3+}$ in different hosts.

\begin{tabular}{lcccc}
\hline \hline \multicolumn{1}{c}{ Host } & $\boldsymbol{\tau}_{\text {rad }}(\boldsymbol{\mu s )}$ & $\boldsymbol{\tau}_{\text {lum }}(\boldsymbol{\mu \mathbf { s } )}$ & $\boldsymbol{\eta}_{\boldsymbol{q}}(\mathbf{\%})$ & Ref. \\
\hline 6-FDA/UVR & & & & \\
$\mathrm{Al}_{2} \mathrm{O}_{3}$ & 218 & 141 & 64.7 & This work \\
$\mathrm{YAG}$ & 474 & 325 & 68.6 & This work \\
$\mathrm{NGAB}$ & 259 & 237 & 91.0 & {$[91]$} \\
$\mathrm{KLa}\left(\mathrm{WO}_{4}\right)_{2}$ & 293 & 58 & 19.7 & {$[92]$} \\
sulphide glass & 685 & 210 & 30.7 & {$[113]$} \\
ZBLAN & 109 & 110 & 100.9 & {$[93]$} \\
phosphate glass & 444 & 494 & 111.3 & {$[94]$} \\
lead silicate glass (SNPZ) & 430 & 190 & 44.2 & {$[48]$} \\
\hline
\end{tabular}




\subsection{Summary}

$\mathrm{Nd}^{3+}$-doped slab and channel waveguides have been fabricated in a polymer and $\mathrm{Al}_{2} \mathrm{O}_{3}$. $\mathrm{Nd}^{3+}$-complex-doped polymer channel waveguides have been realized reproducibly by a simple fabrication procedure mainly based on spin-coating and photo-definition. $\mathrm{Al}_{2} \mathrm{O}_{3}: \mathrm{Nd}^{3+}$ films have been deposited on thermally oxide silicon wafers by reactive co-sputtering, and channel waveguides have been patterned by RIE.

Optical losses in both materials have been studied and compared. $\mathrm{Nd}^{3+}-$ complex-doped 6-FDA/UVR waveguides have a broadband transmission window starting at $550 \mathrm{~nm}$ and extending to around $1100 \mathrm{~nm}$. However, high loss were observed in this polymer waveguides at the wavelength longer than $1100 \mathrm{~nm}$ due to the absorption of polymers. $\mathrm{Al}_{2} \mathrm{O}_{3}: \mathrm{Nd}^{3+}$ waveguides, only the other hand, show a broadband transmission window from visible to telecommunication wavelength.

Absorption bands of $\mathrm{Nd}^{3+}$ have been observed at $580 \mathrm{~nm}, 745 \mathrm{~nm}, 800 \mathrm{~nm}$, and $870 \mathrm{~nm}$, based on which Judd-Ofelt analysis has been applied to study the transition properties of $\mathrm{Nd}^{3+}$ ions in the two hosts.

Photoluminescence spectra of $\mathrm{Nd}^{3+}$ have been experimentally studied and emission around $880 \mathrm{~nm}, 1060 \mathrm{~nm}$, and $1330 \mathrm{~nm}$ is observed, which indicates that $\mathrm{Nd}^{3+}$ ions are active in these two hosts. The experimentally determined luminescence lifetimes show good agreement with the radiative lifetimes calculated from Judd-Ofelt theory.

In conclusion, the optical characterization results indicate that $\mathrm{Nd}^{3+}$ complex-doped 6-FDA/UVR and $\mathrm{Al}_{2} \mathrm{O}_{3}: \mathrm{Nd}^{3+}$ waveguides are well suited for optical amplification and lasing for integrated optical applications. 


\section{Chapter 4}

\section{$\mathrm{Nd}^{3+}$-doped Waveguide Amplifiers}

$\mathrm{Nd}^{3+}$-complex-doped 6-FDA/UVR and $\mathrm{Al}_{2} \mathrm{O}_{3}: \mathrm{Nd}^{3+}$ channel waveguide amplifiers have been experimentally and theoretically investigated at three $\mathrm{Nd}^{3+}$ transitions. In $\mathrm{Nd}^{3+}$-complex-doped, photo-defined polymer channel waveguide amplifiers internal net gain in the range 865-930 $\mathrm{nm}$ was observed and a peak gain of $2.8 \mathrm{~dB}$ at $873 \mathrm{~nm}$ was obtained in a 1.9-cm-long waveguide with a $\mathrm{Nd}^{3+}$ concentration of $0.6 \times 10^{20} \mathrm{~cm}^{-3}$ at a launched pump power of $25 \mathrm{~mW}$. Net gain per unit length at $873 \mathrm{~nm}$ and $1064 \mathrm{~nm}$ of 2.0 $\mathrm{dB} / \mathrm{cm}$ and $5.7 \mathrm{~dB} / \mathrm{cm}$, respectively, has been measured. For $\mathrm{Al}_{2} \mathrm{O}_{3}: \mathrm{Nd}^{3+}$ channel waveguide amplifiers, a maximum gain of $6.3 \mathrm{~dB} / \mathrm{cm}$ at $1064 \mathrm{~nm}$ and $1.93 \mathrm{~dB} / \mathrm{cm}$ at $1330 \mathrm{~nm}$ was observed in a 1.4-cm-long waveguide with a $\mathrm{Nd}^{3+}$ concentration of $1.68 \times 10^{20} \mathrm{~cm}^{-3}$, respectively, at a launched pump power of 45 $\mathrm{mW}$. Optical gain in the range 865-930 $\mathrm{nm}$ was observed and a peak gain of $3.0 \mathrm{~dB}$ at $880 \mathrm{~nm}$ was obtained in 3-and 4.1-cm-long waveguides with $\mathrm{Nd}^{3+}$ concentrations of $1.13 \times 10^{20}$ and $0.65 \times 10^{20} \mathrm{~cm}^{-3}$, respectively, at a launched pump power of $45 \mathrm{~mW}$. By use of a rate-equation model, the gain in the two types of waveguides at each wavelength was calculated and the macroscopic ETU parameter as a function of $\mathrm{Nd}^{3+}$ concentration was derived. The high internal net gain indicates that these two types of $\mathrm{Nd}^{3+}$-doped channel waveguide amplifiers are suitable for providing sufficient gain in many integrated optical devices. 


\subsection{Introduction}

Neodymium-doped waveguide amplifiers are of interest for integrated optical applications at the ion's specific emission wavelengths.

The $\mathrm{Nd}^{3+} 1060$-nm gain transition $\left({ }^{4} \mathrm{~F}_{3 / 2} \rightarrow{ }^{4} \mathrm{I}_{11 / 2}\right)$ possesses a four-levelenergy structure, which provides significant gain at low excitation power for optical amplification and potential lasing. The transition ${ }^{4} \mathrm{~F}_{3 / 2} \rightarrow{ }^{4} \mathrm{I}_{13 / 2}$ around $1330 \mathrm{~nm}$, corresponding to the wavelength of the second standard telecommunication window, is interesting for amplification of optical signals in integrated devices and systems at the telecommunication O-band (1260-1360 nm). The emission on the ${ }^{4} \mathrm{~F}_{3 / 2} \rightarrow{ }^{4} \mathrm{I}_{9 / 2}$ ground-state transition around $880 \mathrm{~nm}$ is important for signal amplification in integrated optical applications, e.g. data transmission in optical interconnects and medical diagnostics. Investigations on the optical power budget for realistic polymerwaveguide based high-speed links via optical backplanes revealed that coupling and routing dependent losses reduce the optical power to a significant extent [1]. Therefore, additional signal amplification within the board is necessary around 850 $\mathrm{nm}$, which is currently considered for optical interconnects in printed circuit boards (PCBs) [2-4] due to the maturity of VCSEL technology at this wavelength. Integration of an amplifying material into optical backplanes can be an efficient solution to maintain a sufficient optical power level. Polymer channel waveguides are compatible with processes used in the PCB industry. In-vivo confocal scanning laser microscopy (CSLM) offers real-time imaging of living tissue at high resolution and high contrast, without physically dissecting the issue $[5,6]$. The wavelength range of $800-900 \mathrm{~nm}$ is of interest for detecting Raman signals back-scattered by water in human skin. Waveguide amplification is of interest for improving the collection of Ramanreflected signals to be analyzed in integrated optical systems.

Optical gain has been reported in rare-earth-ion-doped planar optical waveguides, e.g. in erbium-doped [38, 39] and neodymium-doped [31-33] polymer waveguide amplifiers. A maximum signal enhancement of $8.5 \mathrm{~dB}$ was demonstrated in $\mathrm{Nd}^{3+}$-doped photolime gel polymer planar waveguides [31,32]. After a highly complex fabrication procedure involving a multi-layer architecture, a signal enhancement of $8 \mathrm{~dB}$ at $1060 \mathrm{~nm}$ was obtained in a $\mathrm{NdCl}_{3}$-doped polymer channel waveguide [33]. Polymer-based $\mathrm{Nd}^{3+}$-doped channel waveguide amplifiers that exploit the potential simplicity of polymer deposition and micro-structuring have as yet not been demonstrated.

On the other hand, $\mathrm{Al}_{2} \mathrm{O}_{3}$ has been investigated as a gain material in waveguide structures $[40-42,62-66] . \mathrm{Er}^{3+}$-doped $\mathrm{Al}_{2} \mathrm{O}_{3}$ has been studied as a gain medium and a peak gain of $2 \mathrm{~dB} / \mathrm{cm}$ at $1533 \mathrm{~nm}$ as well as net gain over a wide wavelength range of $80 \mathrm{~nm}$ has been demonstrated [40]. $\mathrm{Al}_{2} \mathrm{O}_{3}$ waveguides doped with $\mathrm{Nd}^{3+}$ are also interesting for optical amplification and lasing.

Although $\mathrm{Nd}^{3+}$-doped waveguide amplifiers on the ${ }^{4} \mathrm{~F}_{3 / 2} \rightarrow{ }^{4} \mathrm{I}_{11 / 2}$ transition have been demonstrated in different types of host materials, there are only few reports on amplification of $\mathrm{Nd}^{3+}$ on the ${ }^{4} \mathrm{~F}_{3 / 2} \rightarrow{ }^{4} \mathrm{I}_{9 / 2}$ and ${ }^{4} \mathrm{~F}_{3 / 2} \rightarrow{ }^{4} \mathrm{I}_{13 / 2}$ transitions with very low optical gain [110].

In the previous Chapter, two types of $\mathrm{Nd}^{3+}$-doped waveguides have been fabricated and characterized. In this chapter, internal net gain in $\mathrm{Nd}^{3+}$-complex-doped 6-FDA/UVR channel waveguide amplifiers at $865-930 \mathrm{~nm}$ and $1064 \mathrm{~nm}$ and in 
$\mathrm{Al}_{2} \mathrm{O}_{3}: \mathrm{Nd}^{3+}$ channel waveguides at $865-930 \mathrm{~nm}, 1064 \mathrm{~nm}$, and $1330 \mathrm{~nm}$ will be discussed.

\subsection{Optical Gain Investigation}

Optical gain was experimentally investigated using a pump-probe method. Small signal gain of $\mathrm{Nd}^{3+}$-complex-doped small-core polymer channel waveguide amplifiers at $840-950 \mathrm{~nm}$ and $1064 \mathrm{~nm}$, and $\mathrm{Al}_{2} \mathrm{O}_{3}: \mathrm{Nd}^{3+}$ single-mode channel waveguide amplifiers at $845-940 \mathrm{~nm}, 1064 \mathrm{~nm}$, and $1330 \mathrm{~nm}$ was measured. The measurement setup is depicted in Fig. 4.1.

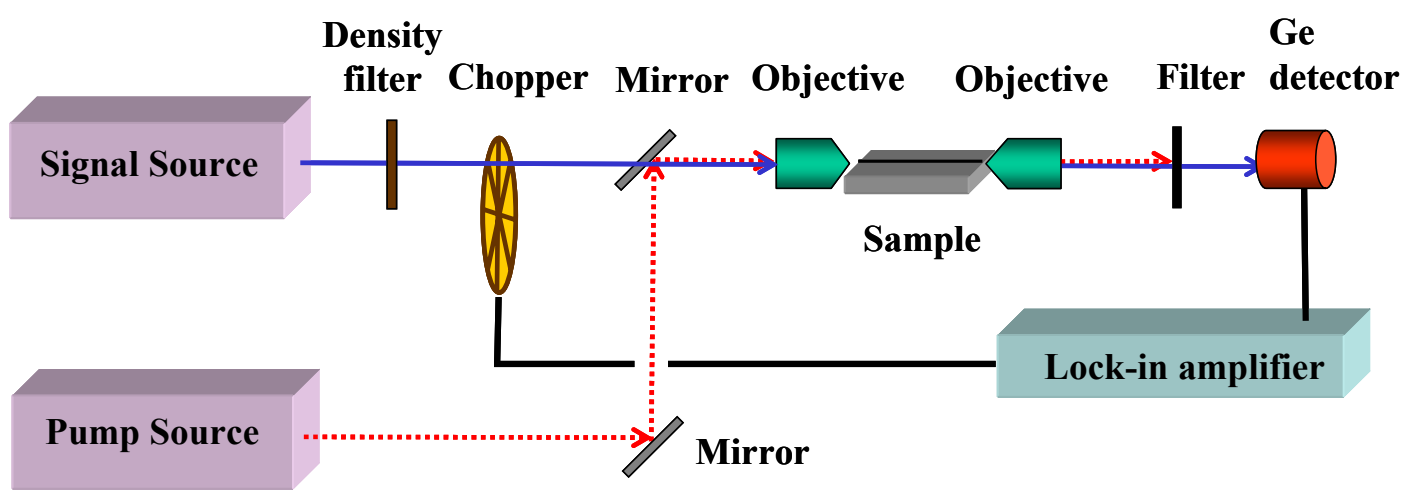

Fig. 4.1. Experimental setup for measuring gain in $\mathrm{Nd}^{3+}$-doped channel waveguides.

A Ti:Sapphire laser operating at $800 \mathrm{~nm}$ was used as the pump source. Diode lasers at $880 \mathrm{~nm}$ and $1330 \mathrm{~nm}$ and a Nd:YAG laser at $1064 \mathrm{~nm}$ were employed as the signal sources. Alternatively, for measuring a gain spectrum at the transition ${ }^{4} \mathrm{~F}_{3 / 2} \rightarrow{ }^{4} \mathrm{I}_{9 / 2}$, the Ti:Sapphire laser tuned around $845-945 \mathrm{~nm}$ was used as the signal source, while an external cavity diode laser operating at $800 \mathrm{~nm}$ was applied as the pump source. After attenuation only a low signal power of typically $1-10 \mu \mathrm{W}$ was launched into the waveguide, which was in the range of a small signal corresponding to the waveguide geometry.

A mechanical chopper was inserted into the signal beam path and connected to a lock-in amplifier. Pump light and modulated signal light were combined by a dichroic mirror, and coupled into and out of the waveguide via microscope objectives. The unabsorbed pump light coupled out of the waveguide was blocked by a high-pass filter (RG830 or RG850), while the transmitted signal light was measured by a germanium photodiode and amplified with the lock-in technique. The optical gain was determined by measuring the ratio of the transmitted signal intensities $I_{p}$ and $I_{u}$ in the pumped and unpumped case, respectively. By subtracting the waveguide background propagation and absorption losses $\alpha(\lambda)(\mathrm{dB} / \mathrm{cm})$ at the signal wavelength, the internal net gain was obtained by calculating the small-signal-gain coefficient in $\mathrm{dB} / \mathrm{cm}$ from the equation

$$
\gamma_{\text {meas }}(\lambda)=10 \cdot \log _{10}\left(I_{p}(\lambda) / I_{u}(\lambda)\right) / l-\alpha(\lambda)
$$


where $l$ is the length of the waveguide channel. This approach eliminates coupling losses which occur in the measurement from the evaluation and provides the gain experienced by the launched signal power along the waveguide. In this way, we considered solely internal net gain in the waveguide amplifiers.

The pump or signal light launched into the waveguide can be estimated by measuring the power of the transmitted light from the output of the channel when tuning the laser source to a wavelength of no $\mathrm{Nd}^{3+}$ absorption. By subtracting the propagation loss at this wavelength, the launched power can be determined. During the gain investigation in $\mathrm{Nd}^{3+}$-complex-doped 6-FDA/UVR waveguides, a maximum launched pump power of $25 \mathrm{~mW}$ was used, while in the late study on gain investigation of $\mathrm{Al}_{2} \mathrm{O}_{3}: \mathrm{Nd}^{3+}$ and laser experimental (Chapter 5) higher pump powers were launched into the waveguides due to the improvement of the laser output.

With the aid of the rate-equation models for the three different peak wavelengths, $880 \mathrm{~nm}, 1060 \mathrm{~nm}$, and $1330 \mathrm{~nm}$, derived in Section 2.3.5, the optical gain in two types of waveguides at each wavelength has been simulated and compared with the measured results. Table 4.1 lists all the experimentally determined spectroscopic parameters of $\mathrm{Nd}^{3+}$-complex-doped 6-FDA/UVR and $\mathrm{Al}_{2} \mathrm{O}_{3}: \mathrm{Nd}^{3+}$ channel waveguides used for the measurements and simulations.

The optical mode profiles and confinement of optical power within the channel waveguides were determined by the finite difference (FD) method with the aid of the FieldDesigner software package (PhoeniX [118]). The FD method is based on discretization of the two-dimension (2D) field equation, and the mode calculation lies in the waveguide cross section plane [119]. It is valid for the calculation of guided modes as well as leaky modes in straight waveguides. The percentage of pump and signal power outside the active region, which does not contribute to the population dynamics, was not taken into account in the simulation. The experimentally determined spectroscopic parameters (Table 4.1) were used in the simulation. The population and propagation equations were solved using 128 longitudinal and 32-80 radial elements. The unknown ETU parameter $W_{\text {ETU }}$ was used as the only fitting parameter in the simulation as discussed in Sections 2.3.3 and 2.3.5.

Table 4.1. Parameters used for simulation of $\mathrm{Nd}^{3+}$-doped 6-FDA/epoxy and $\mathrm{Al}_{2} \mathrm{O}_{3}: \mathrm{Nd}^{3+}$ waveguide amplifiers.

\begin{tabular}{lll}
\hline \hline Parameter & $\mathbf{N d}^{3+}$-doped polymer & $\mathbf{A l}_{\mathbf{2}} \mathbf{O}_{3}: \mathbf{N d}^{3+}$ \\
\hline pump wavelength $\lambda_{p}$ & $800 \mathrm{~nm}$ & $800 \mathrm{~nm}$ \\
signal wavelength $\lambda_{s}$ & $873 / 1064 \mathrm{~nm}$ & $880 / 1064 / 1330 \mathrm{~nm}$ \\
\hline refractive index at $800 \mathrm{~nm}$ & & \\
$n_{\text {core }}$ & $n_{6-\mathrm{FDA} / \mathrm{UVR}}=1.522$ & $n_{\mathrm{Al} 2 \mathrm{O} 3}=1.660$ \\
$n_{\text {cladding }}$ & $n_{\mathrm{CHEP}}=1.508$ & $n_{\mathrm{air}}=1.0$ \\
$n_{\text {lower-cladding }}$ & $n_{\mathrm{SiO} 2}=1.456$ & $n_{\mathrm{SiO} 2}=1.456$ \\
\hline refractive index at $873 / 880 \mathrm{~nm}$ & $873 \mathrm{~nm}$ & $880 \mathrm{~nm}$ \\
$n_{\text {core }}$ & $n_{6-\mathrm{FDA} / \mathrm{UVR}}=1.521$ & $n_{\mathrm{Al2O} 3}=1.659$ \\
$n_{\text {cladding }}$ & $n_{\mathrm{CHEP}}=1.507$ & $n_{\mathrm{air}}=1.0$ \\
$n_{\text {lower-cladding }}$ & $n_{\mathrm{SiO} 2}=1.455$ & $n_{\mathrm{SiO} 2}=1.455$ \\
\hline refractive index at $1064 \mathrm{~nm}$ & & \\
$n_{\text {core }}$ & $n_{6-\mathrm{FDA} / \mathrm{UVR}}=1.518$ & $n_{\mathrm{Al} 2 \mathrm{O} 3}=1.658$ \\
$n_{\text {cladding }}$ & $n_{\mathrm{CHEP}}=1.505$ & $n_{\mathrm{air}}=1.0$
\end{tabular}




\begin{tabular}{|c|c|c|}
\hline$n_{\text {lower-cladding }}$ & $n_{\mathrm{SiO} 2}=1.452$ & $n_{\mathrm{SiO} 2}=1.452$ \\
\hline \multicolumn{3}{|l|}{ refractive index at $1330 \mathrm{~nm}$} \\
\hline$n_{\text {core }}$ & - & $n_{\mathrm{Al} 2 \mathrm{O} 3}=1.649$ \\
\hline$n_{\text {cladding }}$ & - & $n_{\mathrm{air}}=1.0$ \\
\hline$n_{\text {lower-cladding }}$ & - & $n_{\mathrm{SiO} 2}=1.448$ \\
\hline \multicolumn{3}{|l|}{ Confinement factor of slab waveguide } \\
\hline $800 \mathrm{~nm}$ & 0.942 & 0.914 \\
\hline $880 \mathrm{~nm}$ & 0.928 & 0.894 \\
\hline waveguide cross section & $5 \times 5 \mu \mathrm{m}^{2}$ & $0.6 \times 2.0 \mu \mathrm{m}^{2}$ \\
\hline width & $5 \mu \mathrm{m}$ & $0.6 \mu \mathrm{m}$ \\
\hline thickness & $5 \mu \mathrm{m}$ & $2.0 \mu \mathrm{m}$ \\
\hline \multicolumn{3}{|l|}{ calculated mode profiles at $800 \mathrm{~nm}$} \\
\hline horizontal Gaussian beam waist $w_{x}$ & $2.35 \mu \mathrm{m}$ & $1.48 \mu \mathrm{m}$ \\
\hline vertical Gaussian beam waist $w_{y}$ & $2.50 \mu \mathrm{m}$ & $0.35 \mu \mathrm{m}$ \\
\hline Gaussian beam waist for simulation $w$ & $2.42 \mu \mathrm{m}$ & $0.72 \mu \mathrm{m}$ \\
\hline confinement factor $\Gamma$ & 0.89 & 0.94 \\
\hline calculated mode profiles at $873 / 880 \mathrm{~nm}$ & $873 \mathrm{~nm}$ & $880 \mathrm{~nm}$ \\
\hline horizontal Gaussian beam waist $w_{x}$ & $2.45 \mu \mathrm{m}$ & $1.55 \mu \mathrm{m}$ \\
\hline vertical Gaussian beam waist $w_{y}$ & $2.56 \mu \mathrm{m}$ & $0.37 \mu \mathrm{m}$ \\
\hline Gaussian beam waist for simulation $w$ & $2.50 \mu \mathrm{m}$ & $0.76 \mu \mathrm{m}$ \\
\hline confinement factor $\Gamma$ & 0.88 & 0.93 \\
\hline \multicolumn{3}{|l|}{ calculated mode profiles at $1064 \mathrm{~nm}$} \\
\hline horizontal Gaussian beam waist $w_{x}$ & $2.70 \mu \mathrm{m}$ & $1.76 \mu \mathrm{m}$ \\
\hline vertical Gaussian beam waist $w_{y}$ & $2.71 \mu \mathrm{m}$ & $0.42 \mu \mathrm{m}$ \\
\hline Gaussian beam waist for simulation & $2.70 \mu \mathrm{m}$ & $0.86 \mu \mathrm{m}$ \\
\hline confinement factor $\Gamma$ & 0.83 & 0.91 \\
\hline \multicolumn{3}{|l|}{ calculated mode profiles at $1330 \mathrm{~nm}$} \\
\hline horizontal Gaussian beam waist $w_{x}$ & - & $2.17 \mu \mathrm{m}$ \\
\hline vertical Gaussian beam waist $w_{y}$ & - & $0.52 \mu \mathrm{m}$ \\
\hline Gaussian beam waist for simulation $w$ & - & $1.06 \mu \mathrm{m}$ \\
\hline confinement factor $\Gamma$ & - & 0.89 \\
\hline pump power $P_{p}$ & $0-30 \mathrm{~mW}$ & 0 to $50 \mathrm{~mW}$ \\
\hline signal power $P_{s}$ & $1 \mu \mathrm{W}$ & $1 \mu \mathrm{W}$ \\
\hline pump intensity $I_{p}$ & $0-1.07 \times 10^{9} \mathrm{~W} / \mathrm{m}^{2}$ & 0 to $4.08 \times 10^{10} \mathrm{~W} / \mathrm{m}^{2}$ \\
\hline signal intensity $I_{S}$ at $873 / 880 \mathrm{~nm}$ & $3.52 \times 10^{4} \mathrm{~W} / \mathrm{m}^{2}$ & $8.10 \times 10^{5} \mathrm{~W} / \mathrm{m}^{2}$ \\
\hline signal intensity $I_{s}$ at $1064 \mathrm{~nm}$ & $3.22 \times 10^{4} \mathrm{~W} / \mathrm{m}^{2}$ & $7.79 \times 10^{5} \mathrm{~W} / \mathrm{m}^{2}$ \\
\hline signal intensity $I_{S}$ at $1330 \mathrm{~nm}$ & - & $7.71 \times 10^{5} \mathrm{~W} / \mathrm{m}^{2}$ \\
\hline $\mathrm{Nd}^{3+}$ concentration & 0.3 to $2.03 \times 10^{20} \mathrm{~cm}^{-3}$ & 0.65 to $2.95 \times 10^{20} \mathrm{~cm}^{-3}$ \\
\hline pump absorption cross section $\sigma_{a b s}^{p}$ & $3.50 \times 10^{-20} \mathrm{~cm}^{2}$ & $0.79 \times 10^{-20} \mathrm{~cm}^{2}$ \\
\hline signal absorption cross section $\sigma_{a b s}^{s}$ at $873 / 880 \mathrm{~nm}$ & $1.01 \times 10^{-20} \mathrm{~cm}^{2}$ & $0.35 \times 10^{-20} \mathrm{~cm}^{2}$ \\
\hline signal emission cross section $\sigma_{e m}^{s}$ at $873 / 880 \mathrm{~nm}$ & $1.83 \times 10^{-20} \mathrm{~cm}^{2}$ & $0.82 \times 10^{-20} \mathrm{~cm}^{2}$ \\
\hline signal absorption cross section $\sigma_{a b s}^{s}$ at $1064 \mathrm{~nm}$ & 0 & 0 \\
\hline signal emission cross section $\sigma_{e m}^{s}$ at $1064 \mathrm{~nm}$ & $3.78 \times 10^{-20} \mathrm{~cm}^{2}$ & $2.01 \times 10^{-20} \mathrm{~cm}^{2}$ \\
\hline signal absorption cross section $\sigma_{a b s}^{s}$ at $1330 \mathrm{~nm}$ & - & 0 \\
\hline signal emission cross section $\sigma_{e m}^{s}$ at $1330 \mathrm{~nm}$ & - & $0.70 \times 10^{-20} \mathrm{~cm}^{2}$ \\
\hline lifetime $\tau_{4}$ & $141 \mu \mathrm{s}$ & $325 \mu \mathrm{s}$ \\
\hline upconversion parameter $\left(W_{\text {ETU }}\right)$ & free parameter & free parameter \\
\hline
\end{tabular}


For the four-level transitions as well as for the three-level transition in the case of strong inversion, gain peaks are expected at the wavelength of each transition of $\mathrm{Nd}^{3+}$ ions, which corresponds to the peak of the luminescence spectrum. Therefore, the internal net gain was investigated around the three peak wavelengths. At the ground-state transition, the gain spectrum at the whole emission band was studied. Internal net gain with various $\mathrm{Nd}^{3+}$ concentrations at different waveguide lengths was investigated to simultaneously maximize pump-light absorption and minimize reabsorption at the signal wavelength.

\subsection{Optical Gain at $1064 \mathrm{~nm}$}

High gain is expected in $\mathrm{Nd}^{3+}$-doped waveguides around $1064 \mathrm{~nm}$ due to the fourlevel nature and large emission cross section of the ${ }^{4} \mathrm{~F}_{3 / 2} \rightarrow{ }^{4} \mathrm{I}_{11 / 2}$ transition. The luminescence spectra of $\mathrm{Nd}^{3+}$-complex-doped 6-FDA/UVR and $\mathrm{Al}_{2} \mathrm{O}_{3}: \mathrm{Nd}^{3+}$ waveguides have been investigated and reported in Chapter 3. A Nd:YAG laser operating at $1064 \mathrm{~nm}$ was used as signal source. At this wavelength optical gain of channel waveguides was measured and simulated for various geometries.

The internal net gain versus propagation length was measured in both polymer and aluminium oxide channel waveguides with varying $\mathrm{Nd}^{3+}$ concentrations. The results are plotted in Fig. 4.2. A maximum of $8 \mathrm{~dB}$ gain was observed in a 4.40cm-long $\mathrm{Nd}^{3+}$-complex-doped 6-FDA/UVR channel waveguide with a $\mathrm{Nd}^{3+}$ concentration of $1.03 \times 10^{20} \mathrm{~cm}^{-3}$ and launched pump power of $25 \mathrm{~mW}$ (Fig. 4.2(a)), while the maximum gain measured in a 4.25 -cm-long $\mathrm{Al}_{2} \mathrm{O}_{3}: \mathrm{Nd}^{3+}$ channel waveguide with a $\mathrm{Nd}^{3+}$ concentration of $1.13 \times 10^{20} \mathrm{~cm}^{-3}$ was $15 \mathrm{~dB}$ (Fig. 4.2(b)) for a launched pump power of $45 \mathrm{~mW}$. The simulated gain is also given in Fig. 4.2 and agrees with the measured results in both types of waveguides. More pump intensity can be launched into $\mathrm{Al}_{2} \mathrm{O}_{3}$ waveguides than into polymer waveguides because of the higher thermal stability of $\mathrm{Al}_{2} \mathrm{O}_{3}$.

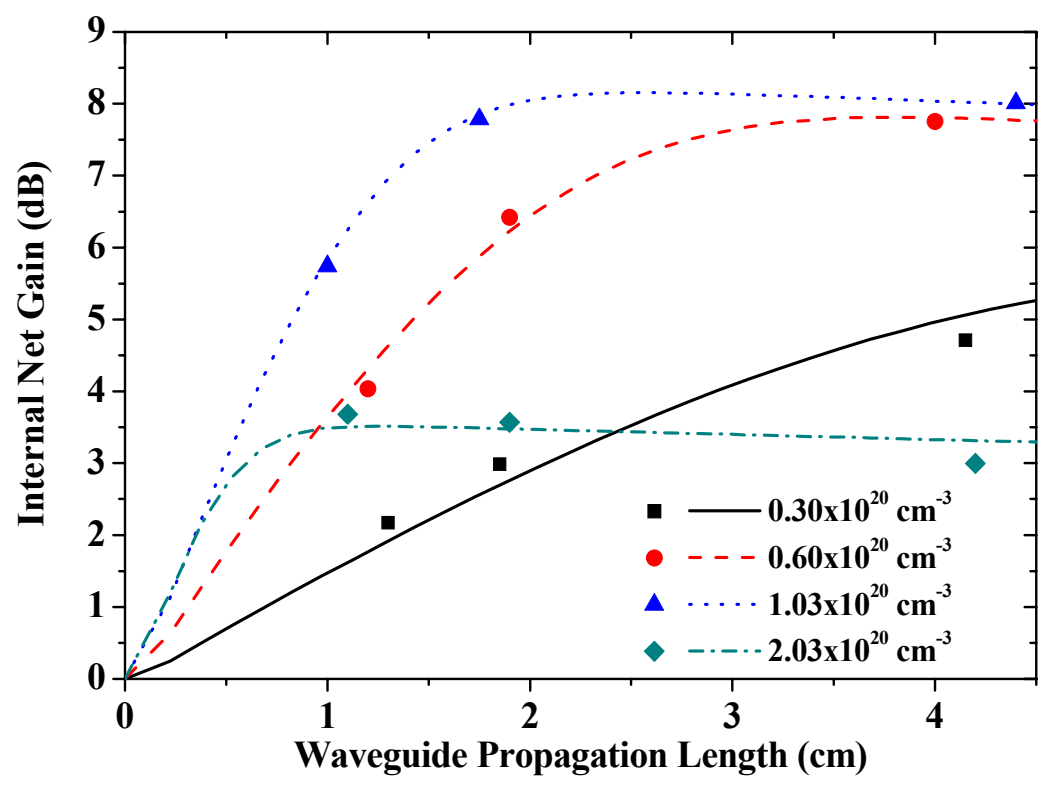

(a) 


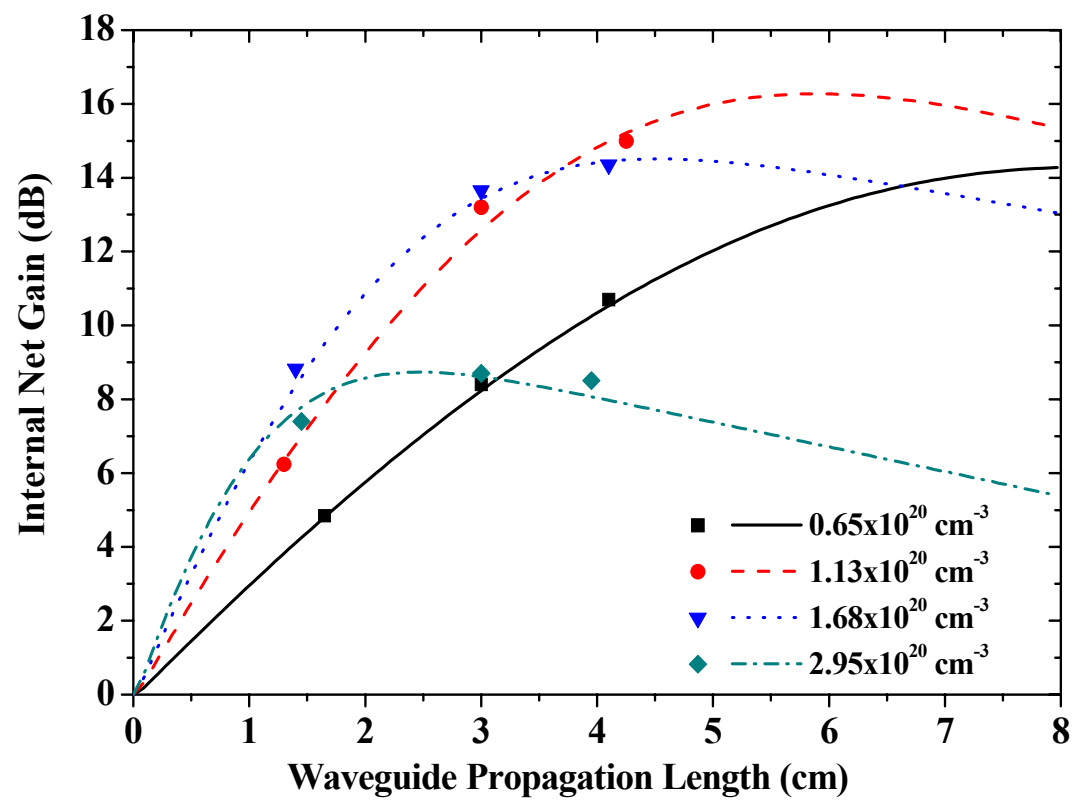

(b)

Fig. 4.2. Measured (dots) and calculated (lines) internal net gain at $1064 \mathrm{~nm}$ versus propagation length of (a) $\mathrm{Nd}^{3+}$-complex-doped 6-FDA/UVR and (b) $\mathrm{Al}_{2} \mathrm{O}_{3}: \mathrm{Nd}^{3+}$ channel waveguides for a launched pump power of $25 \mathrm{~mW}$ and $45 \mathrm{~mW}$, respectively.

Figure 4.3 gives the gain per unit length measured for different $\mathrm{Nd}^{3+}$ concentrations in a short channel length $\left(1.00-1.30 \mathrm{~cm}\right.$ and $1.30-1.65 \mathrm{~cm}$ for $\mathrm{Nd}^{3+}$ doped 6-FDA/UVR and $\mathrm{Al}_{2} \mathrm{O}_{3}: \mathrm{Nd}^{3+}$, respectively) at $1064 \mathrm{~nm}$. The gain per unit length as a function of $\mathrm{Nd}^{3+}$ concentration was also calculated by fitting the ETU parameter and is given in the same figure. At such short lengths the pump power was not completely absorbed, i.e., it became possible to optimize the $\mathrm{Nd}^{3+}$ concentration in each type of waveguide. A maximum gain per unit length of $5.7 \mathrm{~dB} / \mathrm{cm}$ and $6.3 \mathrm{~dB} / \mathrm{cm}$ at $1064 \mathrm{~nm}$ with concentration of $1.03 \times 10^{20} \mathrm{~cm}^{-3}$ and $1.68 \times 10^{20} \mathrm{~cm}^{-3}$ was observed in $\mathrm{Nd}^{3+}$-doped 6-FDA/UVR and $\mathrm{Al}_{2} \mathrm{O}_{3}: \mathrm{Nd}^{3+}$ channel waveguides, respectively. The maximum gain per unit length in the two types of waveguides is comparable when pumped sufficiently. The gain saturation at higher concentrations is mainly due to the ETU processes from ${ }^{4} \mathrm{~F}_{3 / 2}[88,89]$.

Figures 4.4 (a) and (b) show the gain in $\mathrm{dB} / \mathrm{cm}$ versus launched pump power for various channel lengths in the two types of samples with optimal $\mathrm{Nd}^{3+}$ concentration. The measured and simulated gain show a good agreement as a function of launched pump power at all the propagation lengths for the two samples. 


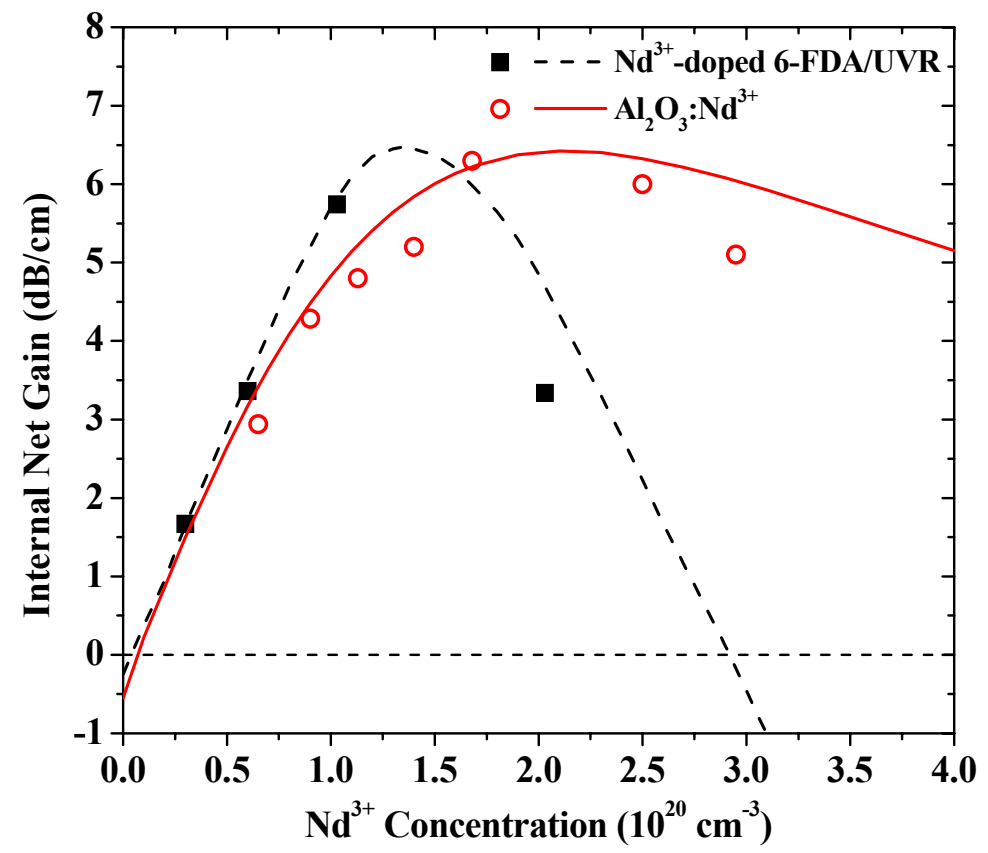

Fig. 4.3. Measured (dots) and calculated (lines) internal net gain per unit length at $1064 \mathrm{~nm}$ for (a) $\mathrm{Nd}^{3+}$-complex-doped 6-FDA/UVR and (b) $\mathrm{Al}_{2} \mathrm{O}_{3}: \mathrm{Nd}^{3+}$ channel waveguides versus $\mathrm{Nd}^{3+}$ concentration for a launched pump power of $25 \mathrm{~mW}$ and $45 \mathrm{~mW}$, respectively.

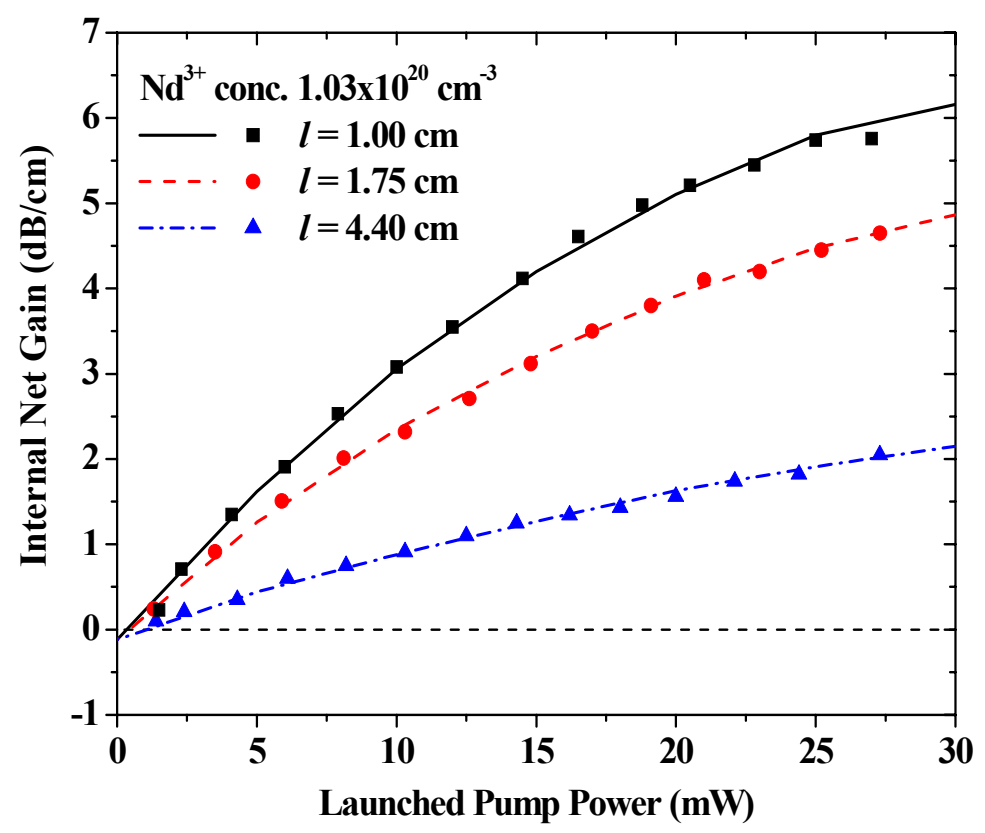

(a) 


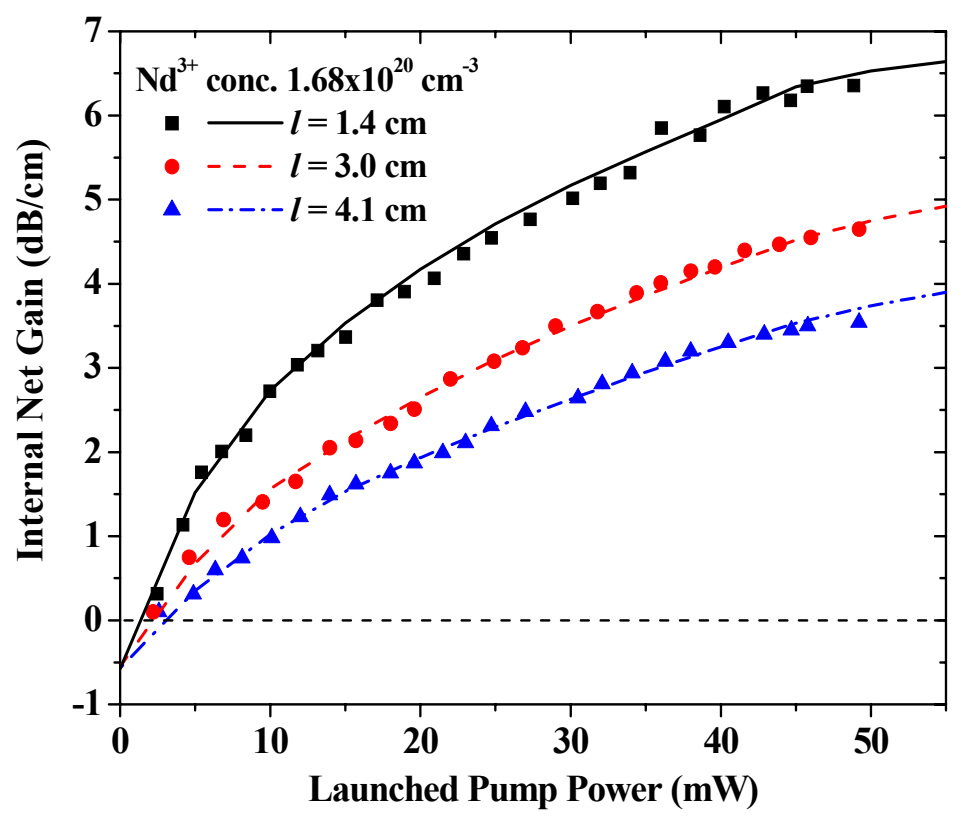

(b)

Fig. 4.4. Measured (dots) and calculated (line) internal net gain per unit length at $1064 \mathrm{~nm}$ versus launched pump power for samples of (a) $\mathrm{Nd}^{3+}$-complex-doped 6-FDA/UVR and (b) $\mathrm{Al}_{2} \mathrm{O}_{3}: \mathrm{Nd}^{3+}$ channel waveguides.

As not all the $\mathrm{Nd}^{3+}$ ions were excited in long samples due to the limitation of launched pump power and concentration-dependent $\mathrm{Nd}^{3+}$ absorption, the gain per unit length, after reaching a maximum, reduced with further increasing propagation length. In the heavily $\mathrm{Nd}^{3+}$-doped samples, the total launched pump power was absorbed completely in the first 2 -cm propagation length for all the samples. In this case, there was no contribution to gain but only extra propagation and absorption losses in the remainder of channel length. Since re-absorption of signal light at 1064 $\mathrm{nm}$ is negligible, higher total gain in $\mathrm{dB}$ can be achieved at this transition in longer samples with sufficient launched pump power. Therefore, the $\mathrm{Nd}^{3+}$ concentrations of $1.03 \times 10^{20} \mathrm{~cm}^{-3}$ and $1.68 \times 10^{20} \mathrm{~cm}^{-3}$ are the optimal $\mathrm{Nd}^{3+}$ concentrations for the maximum gain per unit length at $1064 \mathrm{~nm}$ in our polymer and $\mathrm{Al}_{2} \mathrm{O}_{3}$ channel waveguides at these geometries, respectively.

In conclusion, both waveguide materials have been measured to provide high gain at $1064 \mathrm{~nm}$. The maximum gain measured in $\mathrm{Al}_{2} \mathrm{O}_{3}: \mathrm{Nd}^{3+}$ channel waveguides is higher than that in $\mathrm{Nd}^{3+}$-complex-doped 6-FDA/UVR mainly because of the smaller waveguide geometry and thus higher available pump intensity. However, the polymer waveguides have higher emission cross section and lower propagation loss at $1064 \mathrm{~nm}$, which explains the similar maximum gain in $\mathrm{dB} / \mathrm{cm}$ in the short samples given in Fig. 4.3. The gain at $1064 \mathrm{~nm}$ measured in this work is comparable to that having been reported in polymer and glass-based $\mathrm{Nd}^{3+}$-doped waveguides [31-33, 48].

\subsection{Optical Gain at $1330 \mathrm{~nm}$}

High waveguide losses have been observed in $\mathrm{Nd}^{3+}$-complex-doped 6-FDA/UVR channel waveguides around $1330 \mathrm{~nm}$ due to optical absorption of the residual $\mathrm{C}-\mathrm{H}$ 
bonding in polymer hosts. Thus, these Nd-doped polymer channel waveguides are not suitable materials for optical amplification in the telecom $O$ band (1260-1360 nm).

On the other hand, the propagation loss of $\mathrm{Al}_{2} \mathrm{O}_{3}: \mathrm{Nd}^{3+}$ channel waveguides at $1330 \mathrm{~nm}$ is relatively low, typically $\sim 0.35 \mathrm{~dB} / \mathrm{cm}$. Although the emission peak of the $\mathrm{Nd}^{3+}$ transition ${ }^{4} \mathrm{~F}_{3 / 2} \rightarrow{ }^{4} \mathrm{I}_{13 / 2}$ in $\mathrm{Al}_{2} \mathrm{O}_{3}: \mathrm{Nd}^{3+}$ is at $1340 \mathrm{~nm}$, the gain was measured at $1330 \mathrm{~nm}$. This is because of the availability of a signal source at $1330 \mathrm{~nm}$, at which the emission cross section has a value equaling $75 \%$ of its peak value at $1340 \mathrm{~nm}$.

The internal net gain versus propagation length was measured and simulated by the rate-equation model for $\mathrm{Al}_{2} \mathrm{O}_{3}: \mathrm{Nd}^{3+}$ channel waveguides with various $\mathrm{Nd}^{3+}$ concentrations, the results of which are plotted in Fig. 4.5. A maximum of $5.1 \mathrm{~dB}$ internal net gain was observed in a $4.25-\mathrm{cm}-$ long channel waveguide with a $\mathrm{Nd}^{3+}$ concentration of $1.13 \times 10^{20} \mathrm{~cm}^{-3}$.

Figure 4.6 shows the measured and simulated gain per unit length for seven different $\mathrm{Nd}^{3+}$ concentrations for short waveguide length $(1.30-1.65 \mathrm{~cm})$. The maximum gain per unit length at $1330 \mathrm{~nm}$ was measured at a concentration of $1.68 \times 10^{20} \mathrm{~cm}^{-3}$. Since there is no re-absorption at the ${ }^{4} \mathrm{~F}_{3 / 2} \rightarrow{ }^{4} \mathrm{I}_{13 / 2}$ transition, higher gain is expected at the luminescence peak of $1340 \mathrm{~nm}$, and higher total gain in $\mathrm{dB}$ can be achieved in longer samples with sufficient launched pump power.

The gain in $\mathrm{dB} / \mathrm{cm}$ versus launched pump power in the sample of optimal concentration was investigated with various channel lengths and is given in Fig. 4.7.

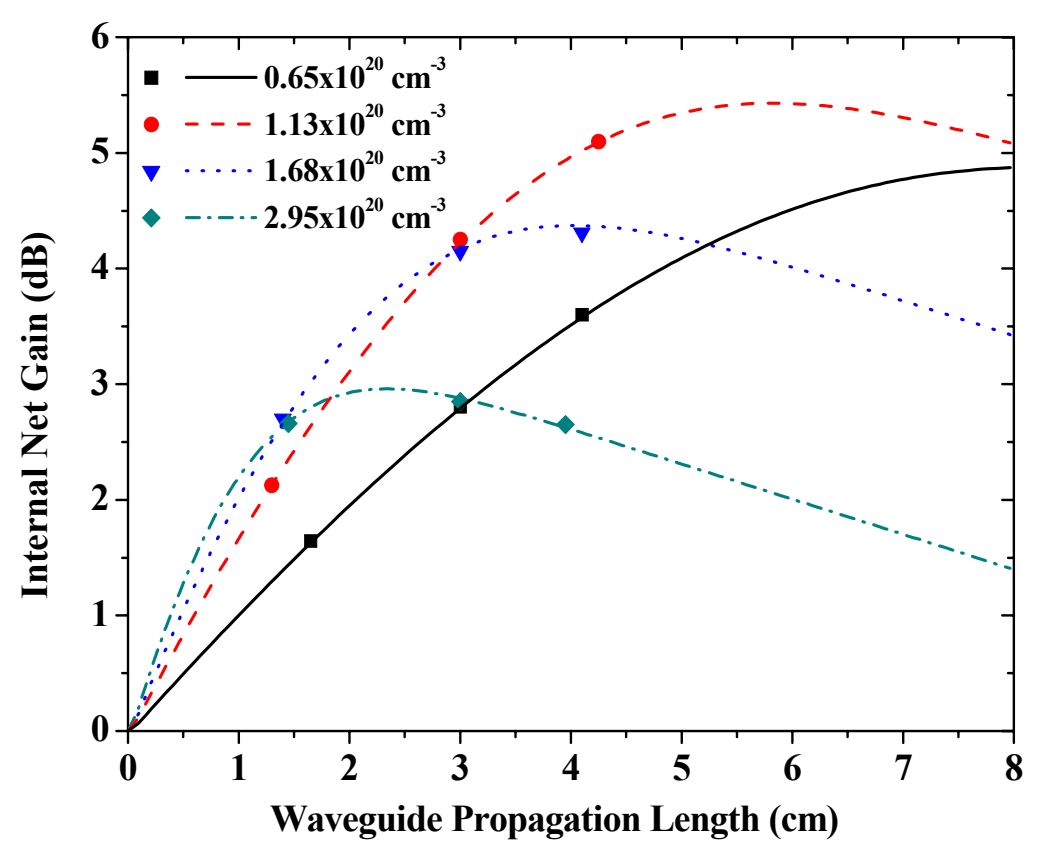

Fig. 4.5. Measured (dots) and calculated (lines) internal net gain at $1330 \mathrm{~nm}$ versus propagation length for a launched pump power of $45 \mathrm{~mW}$ in $\mathrm{Al}_{2} \mathrm{O}_{3}: \mathrm{Nd}^{3+}$ channel waveguides. 


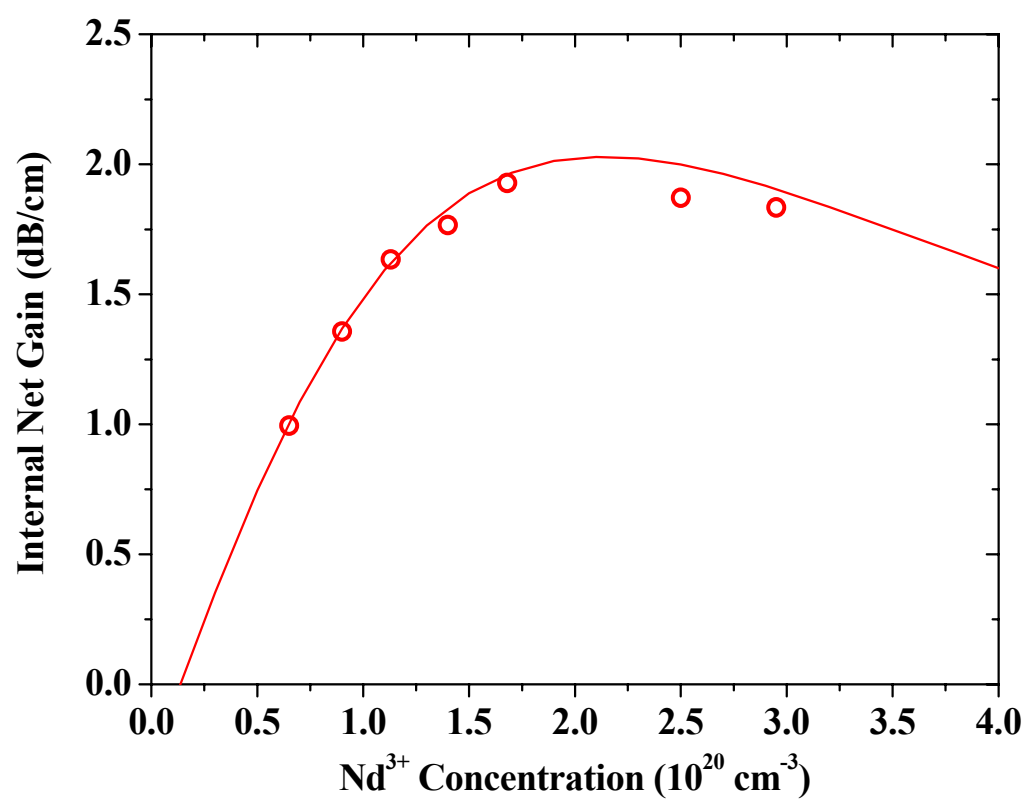

Fig. 4.6. Measured (dots) and calculated (lines) internal net gain per unit length at $1330 \mathrm{~nm}$ versus $\mathrm{Nd}^{3+}$ concentration for a launched pump power of $45 \mathrm{~mW}$ in $\mathrm{Al}_{2} \mathrm{O}_{3}: \mathrm{Nd}^{3+}$ channel waveguides.

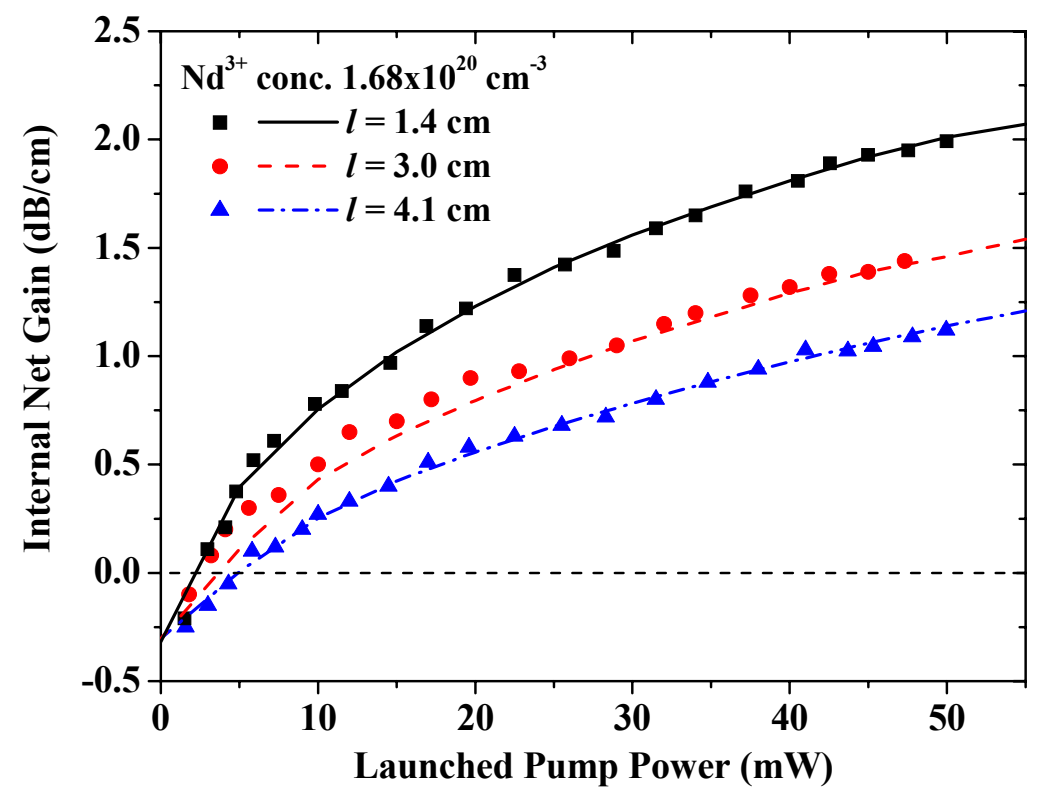

Fig. 4.7. Measured (dots) and calculated (line) internal net gain per unit length at $1330 \mathrm{~nm}$ versus launched pump power in $\mathrm{Al}_{2} \mathrm{O}_{3}: \mathrm{Nd}^{3+}$ channel waveguides for the samples with maximum gain per unit length.

The results indicate that $\mathrm{Al}_{2} \mathrm{O}_{3}: \mathrm{Nd}^{3+}$ channel waveguides are well-suited for amplification in telecommunication around $1330 \mathrm{~nm}$. Since a high-speed $\mathrm{Al}_{2} \mathrm{O}_{3}: \mathrm{Er}^{3+}$ waveguide amplifier at a transmission rate of $170 \mathrm{Gbit} / \mathrm{s}$ has been demonstrated at $1550 \mathrm{~nm}$ [120], high-speed amplification around $1330 \mathrm{~nm}$ in $\mathrm{Al}_{2} \mathrm{O}_{3}: \mathrm{Nd}^{3+}$ channel waveguides is also possible. 


\subsection{Optical Gain at 845-945 $\mathrm{nm}$}

Optical gain was investigated at $873 \mathrm{~nm}$ and $880 \mathrm{~nm}$ in $\mathrm{Nd}^{3+}$-doped 6-FDA/UVR and $\mathrm{Al}_{2} \mathrm{O}_{3}: \mathrm{Nd}^{3+}$ channel waveguides, respectively, corresponding to the luminescence peak of the ${ }^{4} \mathrm{~F}_{3 / 2} \rightarrow{ }^{4} \mathrm{I}_{11 / 2}$ transition in each material.

The measured and simulated internal net gain versus propagation length in the two types of channel waveguides with various $\mathrm{Nd}^{3+}$ concentrations is given in Fig. 4.8. For $\mathrm{Nd}^{3+}$-complex-doped 6-FDA/UVR samples, a maximum gain of $2.7 \mathrm{~dB}$ and $2.8 \mathrm{~dB}$ was demonstrated at $873 \mathrm{~nm}$ with $\mathrm{Nd}^{3+}$ concentrations of 0.3 and $0.6 \times 10^{20} \mathrm{~cm}^{-3}$, respectively, for a launched pump power of $25 \mathrm{~mW}$ (Fig. 4.8(a)). From the results in Fig. 4.8(b), a peak gain of $3 \mathrm{~dB}$ at $880 \mathrm{~nm}$ was obtained in 3.0- and 4.1cm-long $\mathrm{Al}_{2} \mathrm{O}_{3}$ waveguides with $\mathrm{Nd}^{3+}$ concentrations of 1.13 and $0.65 \times 10^{20} \mathrm{~cm}^{-3}$, respectively, at a launched pump power of $45 \mathrm{~mW}$.

Figure 4.9 shows the gain per unit length measured for different $\mathrm{Nd}^{3+}$ concentrations in a short channel length $\left(1.00-1.30 \mathrm{~cm}\right.$ and $1.30-1.65 \mathrm{~cm}$ for $\mathrm{Nd}^{3+}$ doped 6-FDA/UVR and $\mathrm{Al}_{2} \mathrm{O}_{3}: \mathrm{Nd}^{3+}$, respectively). A maximum gain per unit length of $2.0 \mathrm{~dB} / \mathrm{cm}$ was measured at $873 \mathrm{~nm}$ in a $1.0-\mathrm{cm}$-long $\mathrm{Nd}^{3+}$-doped 6-FDA/UVR with $\mathrm{Nd}^{3+}$ concentration of $1.03 \times 10^{20} \mathrm{~cm}^{-3}$, while a maximum of $1.57 \mathrm{~dB} / \mathrm{cm}$ gain was measured at $880 \mathrm{~nm}$ in an $\mathrm{Al}_{2} \mathrm{O}_{3}: \mathrm{Nd}^{3+}$ channel waveguide with concentration of $1.40 \times 10^{20} \mathrm{~cm}^{-3}$.

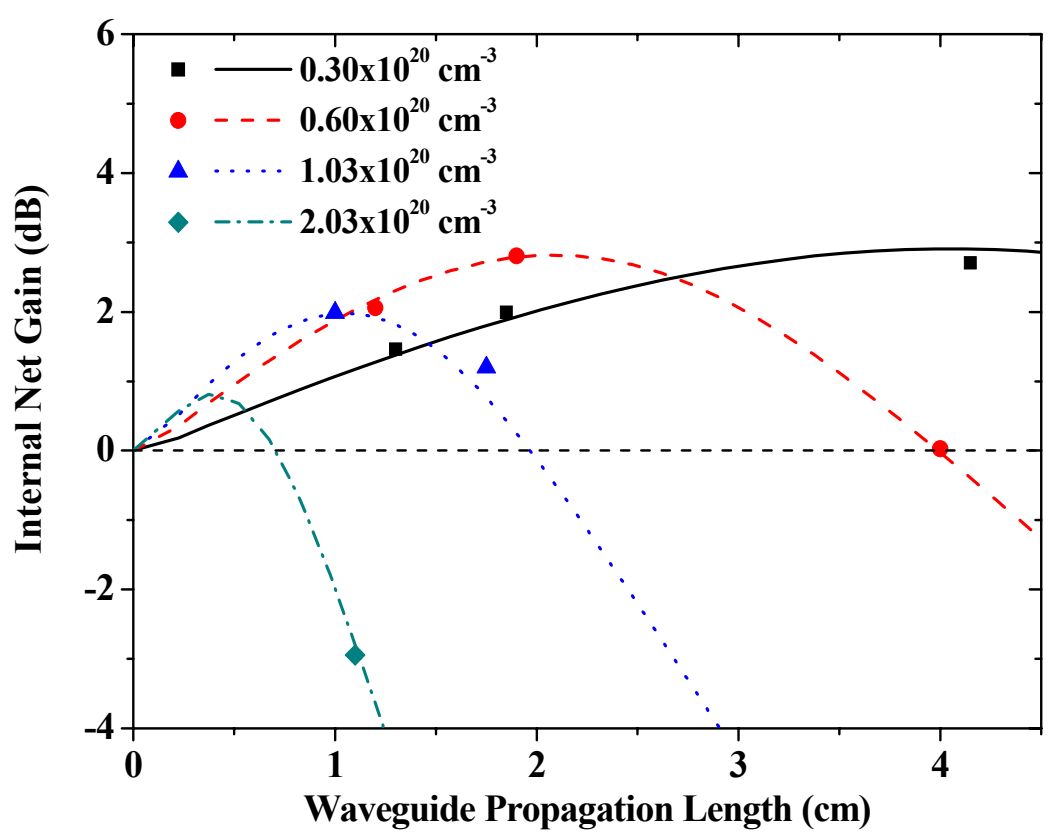

(a) 


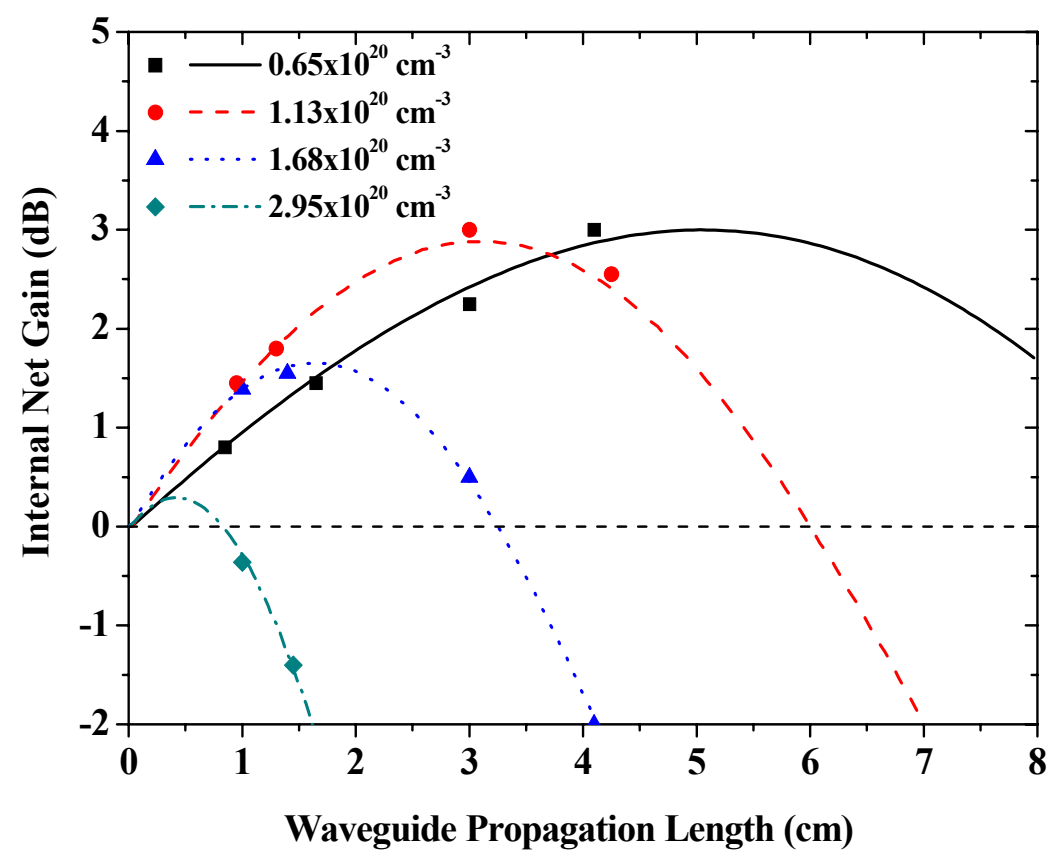

(b)

Fig. 4.8. Measured (dots) and calculated (lines) internal net gain versus propagation length of (a) $\mathrm{Nd}^{3+}$ complex-doped 6-FDA/UVR channel waveguides at $873 \mathrm{~nm}$ and (b) $\mathrm{Al}_{2} \mathrm{O}_{3}: \mathrm{Nd}^{3+}$ channel waveguides at $880 \mathrm{~nm}$ for a launched pump power of $25 \mathrm{~mW}$ and $45 \mathrm{~mW}$, respectively.

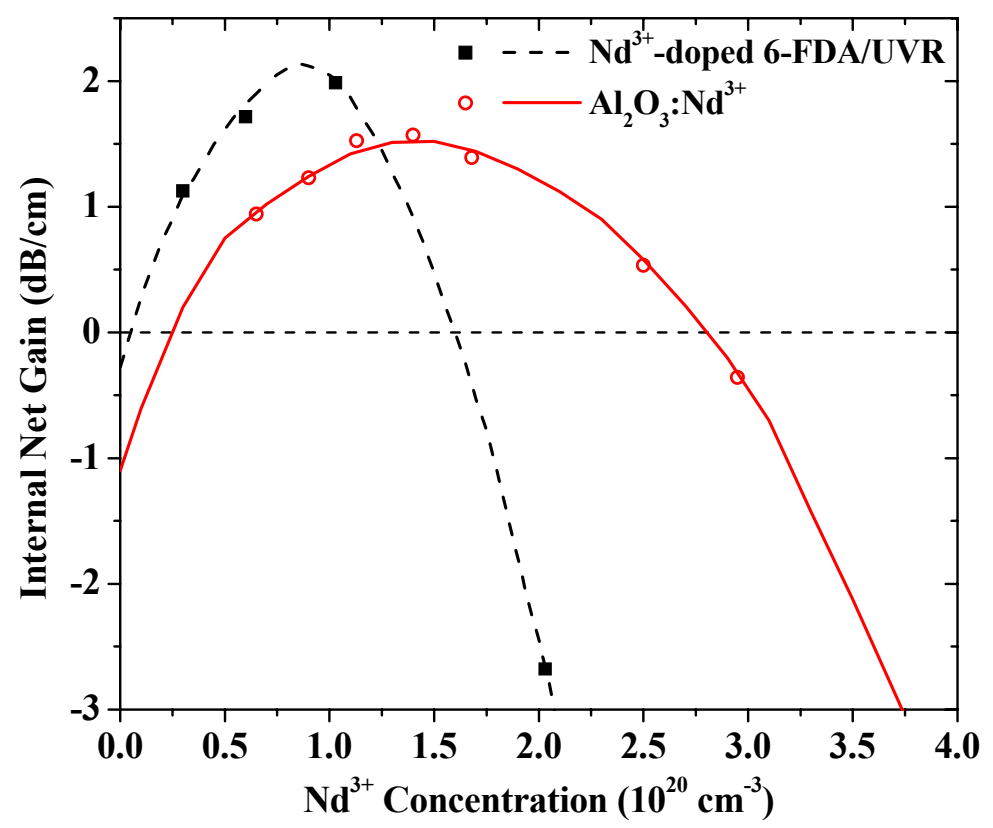

Fig. 4.9. Measured (dots) and calculated (lines) internal net gain per unit length in (a) $\mathrm{Nd}^{3+}$-complexdoped 6-FDA/UVR channel waveguides at $873 \mathrm{~nm}$ and (b) $\mathrm{Al}_{2} \mathrm{O}_{3}: \mathrm{Nd}^{3+}$ channel waveguides at $880 \mathrm{~nm}$ versus $\mathrm{Nd}^{3+}$ concentration for a launched pump power of $25 \mathrm{~mW}$ and $45 \mathrm{~mW}$, respectively. 
The gain in $\mathrm{dB} / \mathrm{cm}$ as a function of launched pump power in the two samples with optimal $\mathrm{Nd}^{3+}$ concentration in each type of waveguide is given in Figs. 4.10 (a) and (b), respectively.

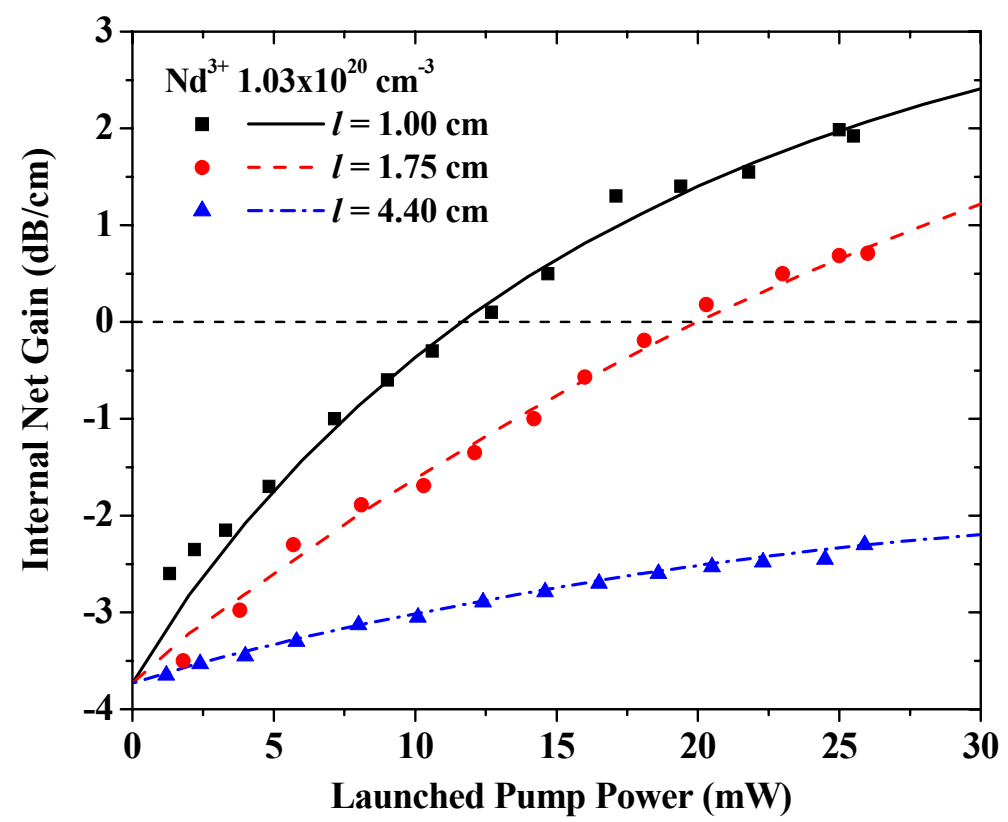

(a)

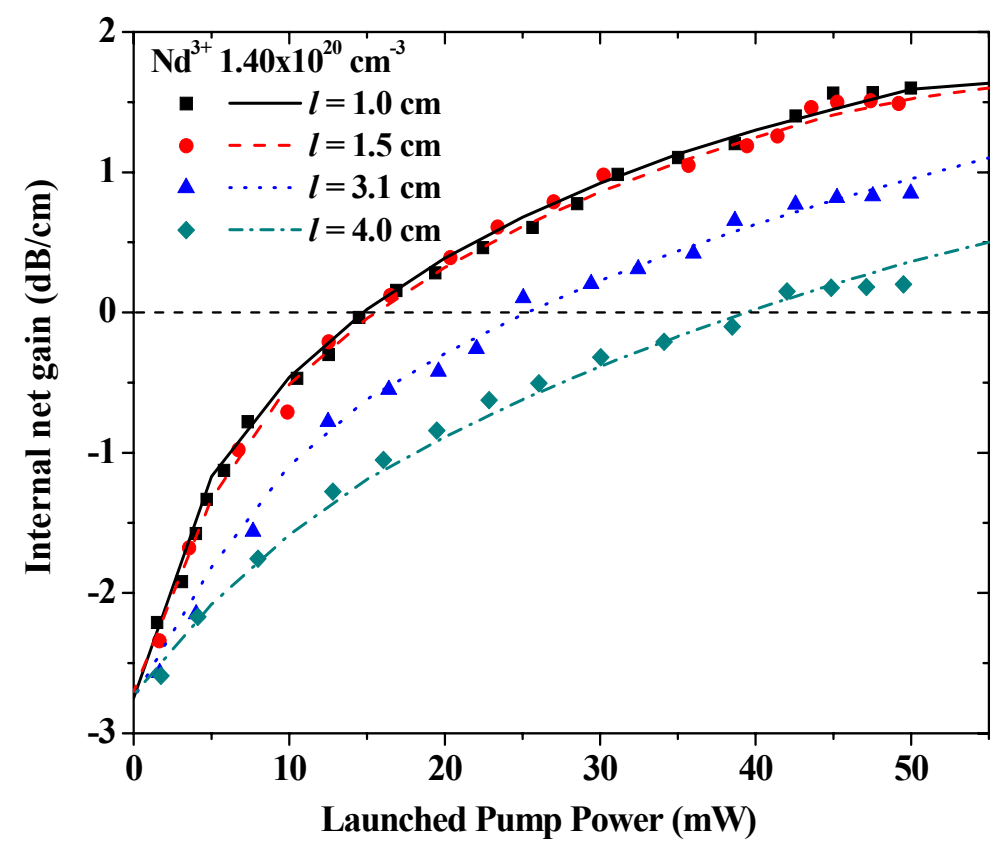

(b)

Fig. 4.10. Measured (dots) and calculated (line) internal net gain per unit length versus launched pump power for the samples of (a) $\mathrm{Nd}^{3+}$-complex-doped 6-FDA/UVR at $873 \mathrm{~nm}$ and (b) $\mathrm{Al}_{2} \mathrm{O}_{3}: \mathrm{Nd}^{3+}$ channel waveguides at $880 \mathrm{~nm}$, respectively, having maximum gain per unit length in Fig. 4.9.

As discussed in the previous two sections, there is no signal re-absorption around $1060 \mathrm{~nm}$ and $1330 \mathrm{~nm}$, therefore higher total gain can be achieved at these two 
transitions in longer samples with sufficient launched pump power. However, for gain at the $\mathrm{Nd}^{3+}$ ground-state transition, although a maximum gain of $2 \mathrm{~dB} / \mathrm{cm}$ and $1.57 \mathrm{~dB} / \mathrm{cm}$ can be reached at the luminescence peak in short samples of $\mathrm{Nd}^{3+}$ complex-doped 6-FDA/UVR and $\mathrm{Al}_{2} \mathrm{O}_{3}: \mathrm{Nd}^{3+}$ channel waveguide, respectively, the total optical gain drops significantly when increasing the channel length owing to strong re-absorption of signal light by $\mathrm{Nd}^{3+}$ ions in their ground state and reduced propagating pump power. The re-absorption of $\mathrm{Nd}^{3+}$ must be taken into account when studying and optimizing the gain at the $\mathrm{Nd}^{3+}$ ground-state transition. Therefore, in order to optimize the optical gain of $\mathrm{Nd}^{3+}$-doped waveguides at the ground-state transition, it is necessary to study the absorption and emission spectrum at this wavelength.

The optical gain spectrum at $845-945 \mathrm{~nm}$ in the two types of waveguides with various $\mathrm{Nd}^{3+}$ concentrations was investigated. Figure 4.11 (a) displays the internal net gain per unit length measured with channel lengths around $1.0-1.3 \mathrm{~cm}$ in $\mathrm{Nd}^{3+}$-complex-doped 6-FDA/UVR channel waveguides at a launched pump power of $25 \mathrm{~mW}$, and the obtained gain bandwidth extends from $865 \mathrm{~nm}$ to $930 \mathrm{~nm}$. Figure 4.11 (b) shows the measured internal net gain spectra in $\mathrm{dB} / \mathrm{cm}$ in $\mathrm{Al}_{2} \mathrm{O}_{3}: \mathrm{Nd}^{3+}$ channel waveguides with channel lengths around $0.85-1.00 \mathrm{~cm}$ at a launched pump power of $45 \mathrm{~mW}$, and the broadest gain bandwidth observed was $865-930 \mathrm{~nm}$.

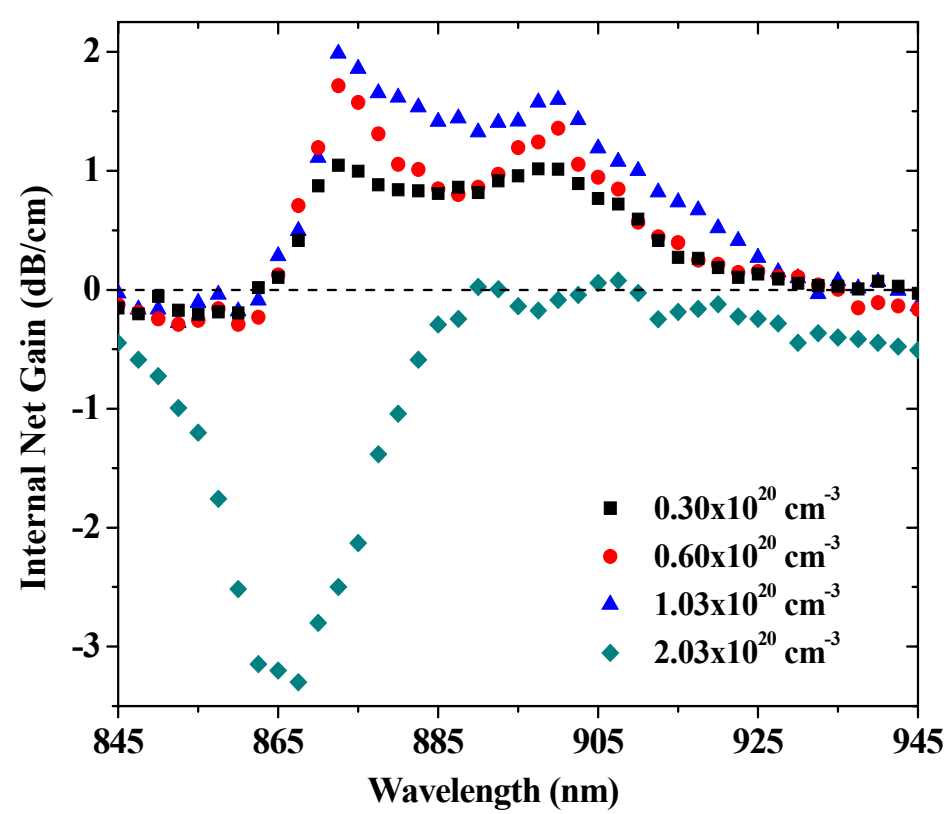

(a) 


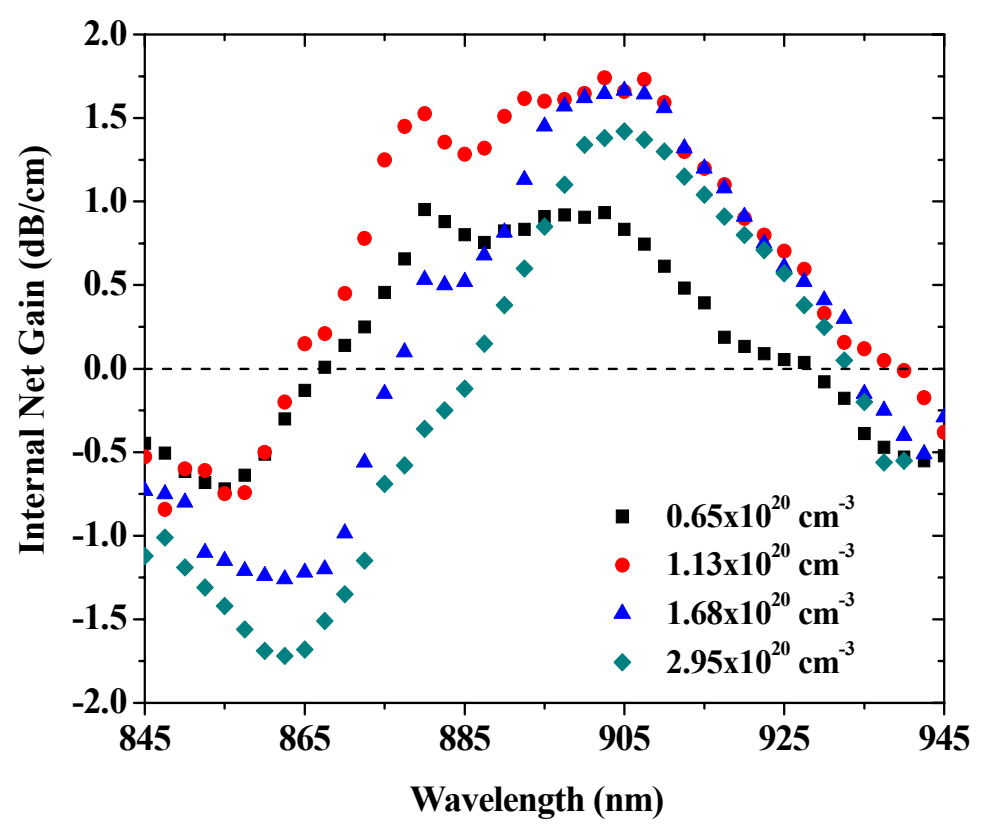

(b)

Fig. 4.11. Internal net gain spectrum at $845-945 \mathrm{~nm}$ versus $\mathrm{Nd}^{3+}$ concentration in (a) $\mathrm{Nd}^{3+}$-complexdoped 6-FDA/UVR channel waveguides for a launched pump power of $25 \mathrm{~mW}$ and (b) $\mathrm{Al}_{2} \mathrm{O}_{3}: \mathrm{Nd}^{3+}$ channel waveguides for a launched pump power of $45 \mathrm{~mW}$.

As explained in Chapter 2, the gain cross section $\sigma_{\text {gain }}(\lambda)$ can be calculated from the absorption and emission cross section (see Eq. 2.17). By introducing the fraction of excited $\mathrm{Nd}^{3+}$ ions, $\beta$, Eq. 2.16 can be written as follows:

$$
\sigma_{\text {gain }}(\lambda)=\beta \cdot \sigma_{\text {em }}(\lambda)-(1-\beta) \cdot \sigma_{\text {abs }}(\lambda),
$$

The absorption and emission cross section, $\sigma_{a b s}(\lambda)$ and $\sigma_{e m}(\lambda)$, of $\mathrm{Nd}^{3+}$ in two waveguides can be derived from the measured spectra as described in Chapter 3. Figures 4.12(a) and 4.13(a) show the absorption and emission cross sections at 840$950 \mathrm{~nm}$ of $\mathrm{Nd}^{3+}$-complex-doped 6-FDA/epoxy and $\mathrm{Al}_{2} \mathrm{O}_{3}: \mathrm{Nd}^{3+}$ waveguides, respectively. The peak absorption and emission cross sections of $\mathrm{Nd}^{3+}$-complex-doped 6-FDA/UVR at $873 \mathrm{~nm}$ are 1.01 and $1.83 \times 10^{-20} \mathrm{~cm}^{2}$, respectively, while the peak absorption and emission cross sections in $\mathrm{Al}_{2} \mathrm{O}_{3}: \mathrm{Nd}^{3+}$ at $880 \mathrm{~nm}$ are 0.35 and 0.82 $\times 10^{-20} \mathrm{~cm}^{2}$, respectively. The calculated gain cross sections of two types of waveguides with various fractions of excited $\mathrm{Nd}^{3+}$ ions $\beta$ are given in Fig 4.12(b) and Fig. 4.13(b), respectively. 


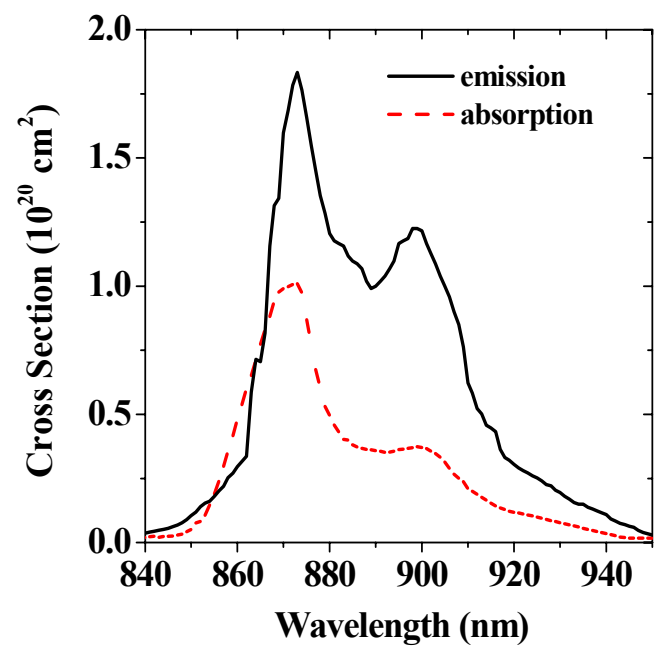

(a)

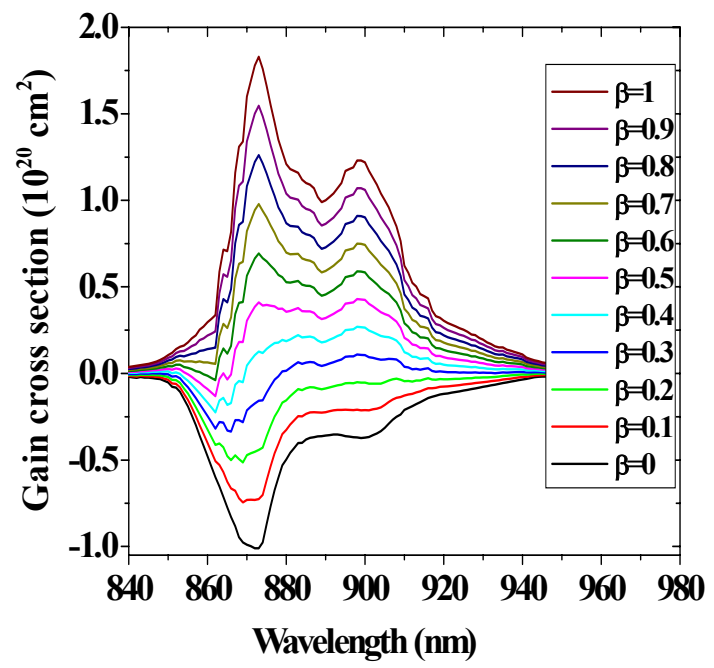

(b)

Fig. 4.12. (a) Emission (solid line), absorption (dash line) cross section and (b) calculated gain cross section at $840-950 \mathrm{~nm}$ of the ${ }^{4} \mathrm{~F}_{3 / 2} \leftrightarrow{ }^{4} \mathrm{I}_{9 / 2}$ ground-state transition in $\mathrm{Nd}^{3+}$-complex-doped 6-FDA/UVR.

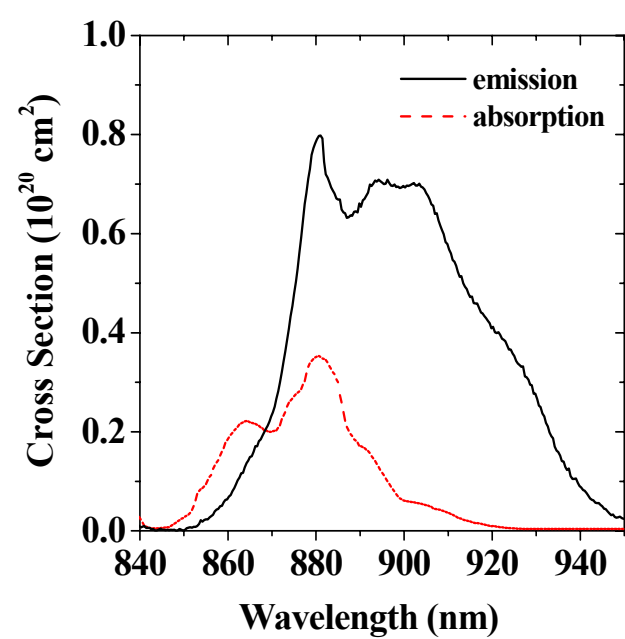

(a)

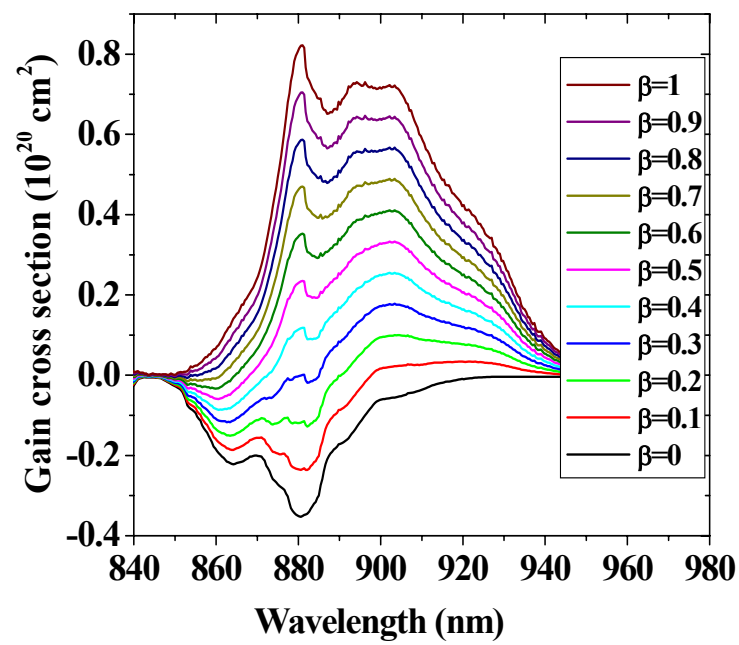

(b)

Fig. 4.13. (a) Emission (solid line), absorption (dash line) cross section and (b) calculated gain cross section at 840-950 $\mathrm{nm}$ of the ${ }^{4} \mathrm{~F}_{3 / 2} \leftrightarrow{ }^{4} \mathrm{I}_{9 / 2}$ ground-state transition in $\mathrm{Al}_{2} \mathrm{O}_{3}: \mathrm{Nd}^{3+}$.

In order to compare the calculated and measured internal net gain spectra, the gain cross section in $\mathrm{cm}^{2}$ was converted to the calculated internal gain coefficient in $\mathrm{dB} / \mathrm{cm}$ using the equation

$$
\gamma_{\text {calc }}(\lambda)=\sigma_{\text {gain }}^{s} \cdot 10 \cdot \log (e) \cdot N_{0}
$$

Figures 4.14(a) and (b) show the internal net gain spectrum calculated for different excitation fractions $\beta$ and measured in waveguides with different channel lengths, for a $\mathrm{Nd}^{3+}$ concentration of $1.03 \times 10^{20} \mathrm{~cm}^{-3}$ and a launched pump power of 25 $\mathrm{mW}$ in $\mathrm{Nd}^{3+}$-doped 6-FDA/UVR waveguides, and a $\mathrm{Nd}^{3+}$ concentration of $1.13 \times 10^{20}$ 
$\mathrm{cm}^{-3}$ and a launched pump power of $45 \mathrm{~mW}$ in $\mathrm{Al}_{2} \mathrm{O}_{3}: \mathrm{Nd}^{3+}$ waveguides, respectively. The measured and calculated internal net gain spectra show good agreement at excitation fractions $\beta$ of $0.55,0.45$, and 0.20 for sample lengths of $1.0,1.8$, and 4.4 $\mathrm{cm}$, respectively, in 6-FDA/UVR waveguides, and at excitation fractions $\beta$ of 0.72 , $0.69,0.62$, and 0.54 for sample lengths of $0.95,1.30,3.00$, and $4.25 \mathrm{~cm}$, respectively, in $\mathrm{Al}_{2} \mathrm{O}_{3}: \mathrm{Nd}^{3+}$ waveguides.

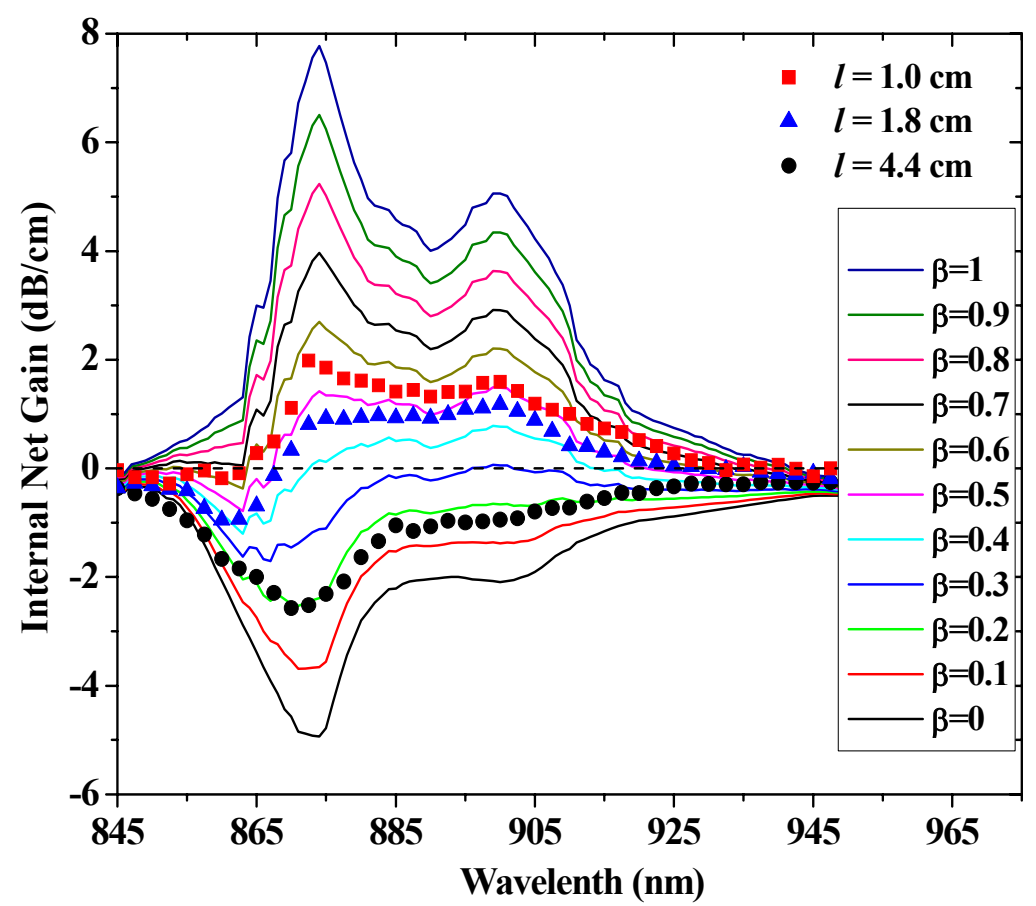

(a)

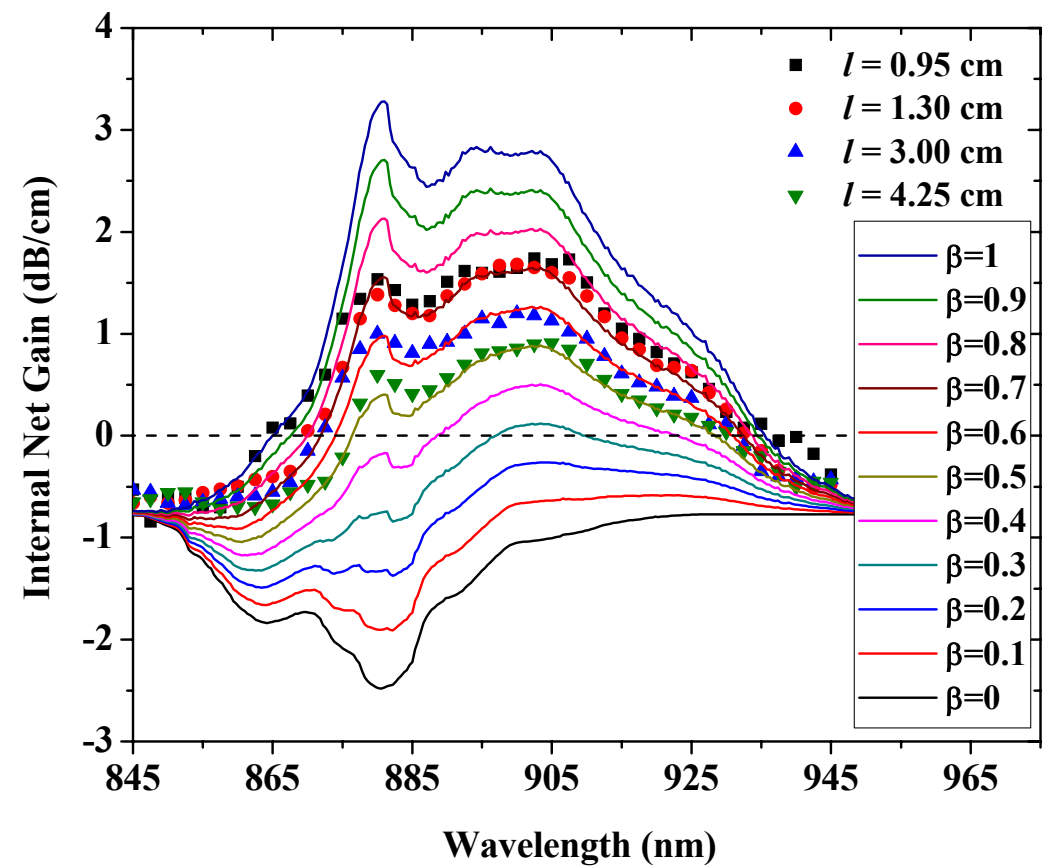

(b)

Fig. 4.14. Measured (dots) and calculated (lines) internal net gain at $845-945 \mathrm{~nm}$ for a $\mathrm{Nd}^{3+}$ concentration of (a) $1.03 \times 10^{20} \mathrm{~cm}^{-3}$ in 6-FDA/UVR for a launched pump power of $25 \mathrm{~mW}$ and (b) $1.13 \times 10^{20} \mathrm{~cm}^{-3}$ in $\mathrm{Al}_{2} \mathrm{O}_{3}$ for a launched pump power of $45 \mathrm{~mW}$. 
Table 4.2 provides the measured gain and fitted excitation fraction of waveguides with different lengths and $\mathrm{Nd}^{3+}$ concentrations in the two types of waveguides. $\mathrm{Al}_{2} \mathrm{O}_{3}$ waveguides have higher excitation fraction than 6-FDA/UVR waveguides with similar $\mathrm{Nd}^{3+}$ concentrations, due to the smaller geometries and higher pump intensities.

Table 4.2. Measured gain and fitted excitation of (a) Nd-doped 6-FDA/UVR and (b) $\mathrm{Al}_{2} \mathrm{O}_{3}: \mathrm{Nd}^{3+}$ waveguides at ground-state transition for a launched pump power of $25 \mathrm{~mW}$ and $45 \mathrm{~mW}$, respectively.

(a)

\begin{tabular}{cccc}
\hline \hline $\begin{array}{c}\mathbf{N d}^{\mathbf{3 +}} \text { concentration } \boldsymbol{N}_{\mathbf{0}} \\
\mathbf{( 1 0}^{\mathbf{2 0}} \mathbf{c m}^{\mathbf{- 3}} \mathbf{)}\end{array}$ & $\begin{array}{c}\text { Channel length } \boldsymbol{l} \\
(\mathbf{c m})\end{array}$ & $\begin{array}{c}\text { Gain coeff. } \boldsymbol{\gamma} \\
\mathbf{( d B / c m )}\end{array}$ & $\begin{array}{c}\text { Excitation fraction } \boldsymbol{\beta} \\
{[\mathbf{\%}]}\end{array}$ \\
\hline \multirow{3}{*}{0.30} & 1.30 & 1.125 & 67 \\
& 1.85 & 1.077 & 66 \\
& 4.15 & 0.653 & 53 \\
\hline \multirow{2}{*}{0.60} & 1.20 & 1.715 & 60 \\
& 1.90 & 1.476 & 57 \\
& 4.00 & 0.007 & 42 \\
\hline \multirow{2}{*}{1.03} & 1.00 & 1.986 & 55 \\
& 1.75 & 0.686 & 45 \\
& 4.40 & -2.500 & 20 \\
\hline & 1.10 & -2.678 & 25 \\
& 1.90 & -5.105 & 75 \\
\hline
\end{tabular}

(b)

\begin{tabular}{|c|c|c|c|}
\hline $\begin{array}{c}\mathrm{Nd}^{3+} \text { concentration } N_{0} \\
\left(10^{20} \mathrm{~cm}^{-3}\right)\end{array}$ & $\begin{array}{c}\text { Channel length } l \\
\text { (cm) }\end{array}$ & $\begin{array}{c}\text { Gain coeff. } \gamma \\
(\mathrm{dB} / \mathrm{cm})\end{array}$ & $\begin{array}{c}\text { Excitation fraction } \beta \\
{[\%]}\end{array}$ \\
\hline \multirow{4}{*}{0.65} & 0.85 & 0.94 & 82.5 \\
\hline & 1.65 & 0.91 & 81 \\
\hline & 3.00 & 0.75 & 77 \\
\hline & 4.10 & 0.73 & 75 \\
\hline \multirow{4}{*}{0.90} & 1.00 & 1.23 & 76 \\
\hline & 1.40 & 1.16 & 74.5 \\
\hline & 3.00 & 0.93 & 69 \\
\hline & 4.10 & 0.61 & 61.5 \\
\hline \multirow{4}{*}{1.13} & 0.95 & 1.53 & 72 \\
\hline & 1.30 & 1.38 & 69 \\
\hline & 3.00 & 1.00 & 62 \\
\hline & 4.25 & 0.60 & 54 \\
\hline \multirow{4}{*}{1.40} & 1.00 & 1.57 & 66 \\
\hline & 1.50 & 1.40 & 63 \\
\hline & 3.05 & 0.82 & 54 \\
\hline & 4.00 & 0.18 & 44.5 \\
\hline \multirow{4}{*}{1.68} & 1.00 & 1.39 & 57.5 \\
\hline & 1.40 & 1.11 & 54 \\
\hline & 3.00 & 0.17 & 42 \\
\hline & 4.10 & -0.49 & 34 \\
\hline
\end{tabular}




\begin{tabular}{cccc}
\hline \multirow{3}{*}{2.50} & 1.05 & 0.53 & 41 \\
& 1.40 & 0.26 & 38.5 \\
& 3.10 & -1.52 & 24 \\
& 4.00 & -2.30 & 18 \\
\hline \multirow{2}{*}{2.95} & 1.00 & -0.36 & 35.5 \\
& 1.45 & -0.97 & 28 \\
& 3.00 & -2.72 & 6 \\
& 3.95 & -3.22 & 1 \\
\hline
\end{tabular}

The excitation fraction can be calculated by the rate-equation model as well (lines in Fig. 4.15). The values of the excitation fraction calculated by the rateequation model shows good agreement with those derived from gain spectra (dots in Fig. 4.15) in both types of waveguides.

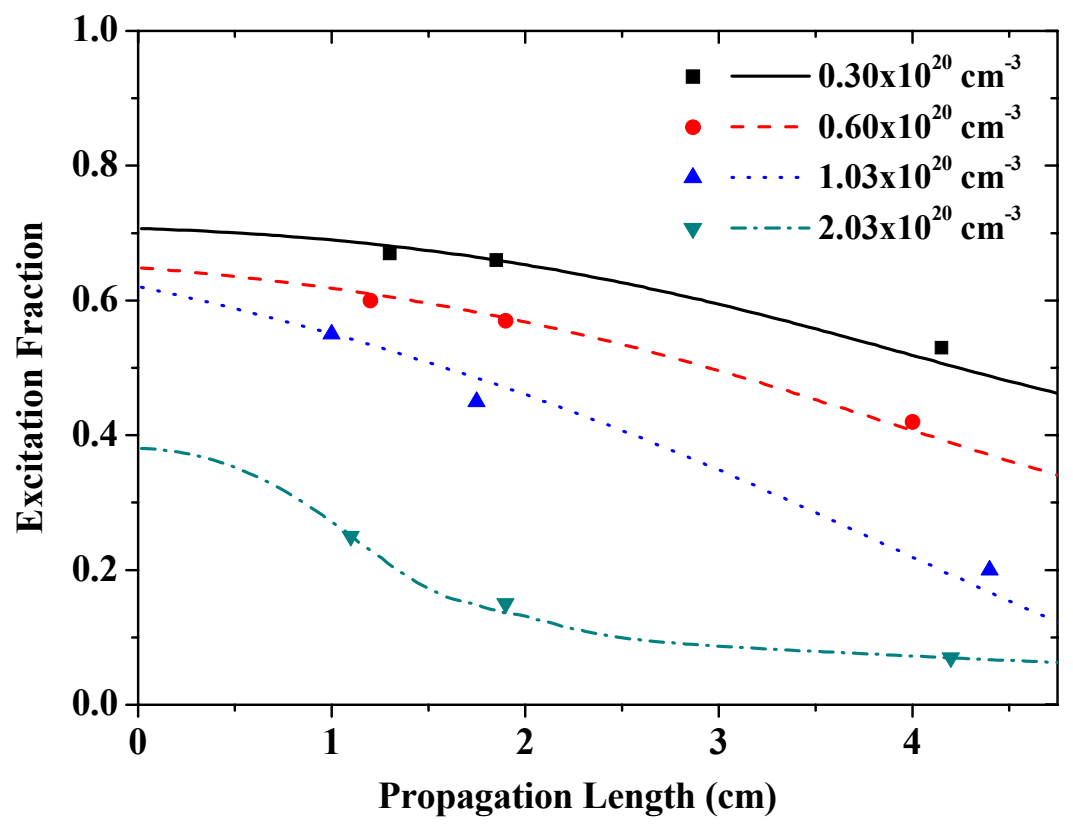

(a) 


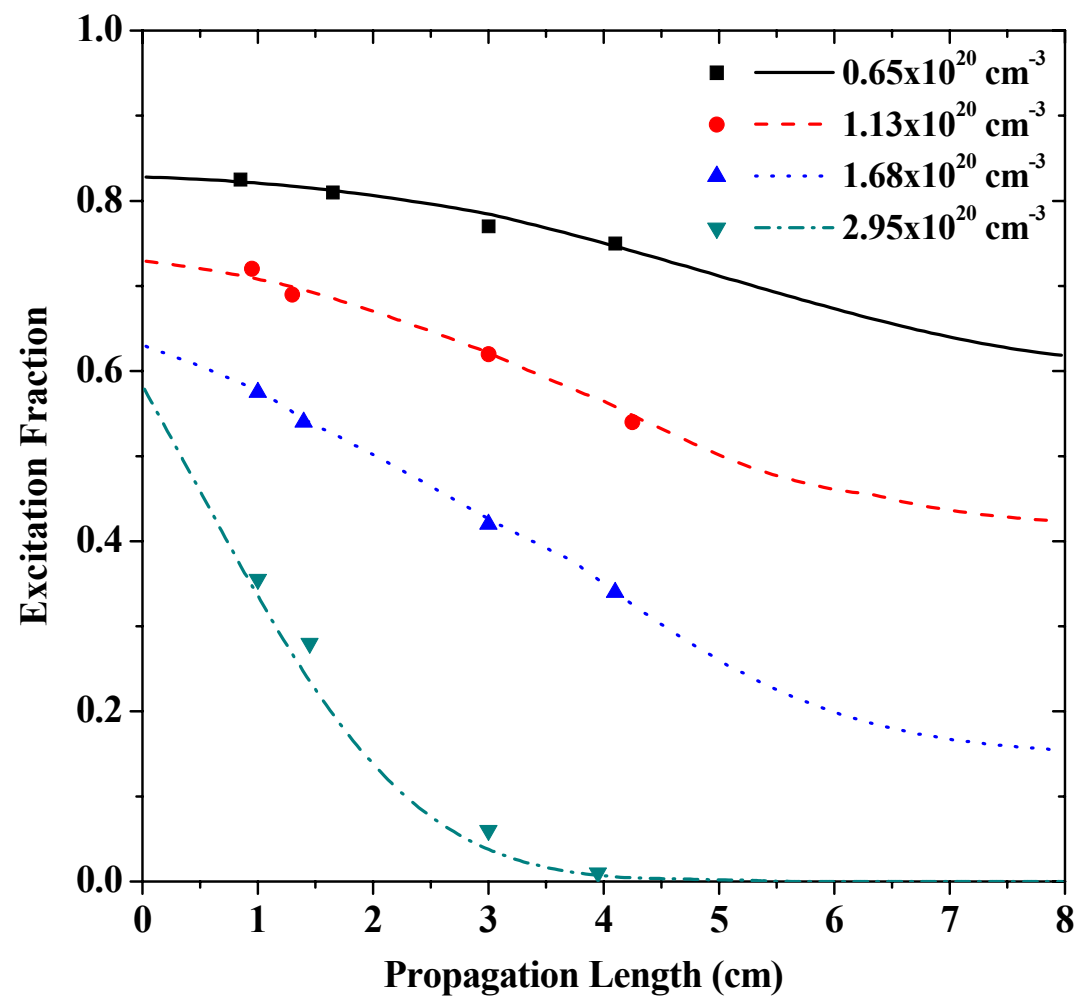

(b)

Fig. 4.15. Population excitation fraction derive from gain spectra (dots) and rate-equation model (lines) as a function of propagation length with various $\mathrm{Nd}^{3+}$ concentrations at wavelength range of 865-930 $\mathrm{nm}$ in (a) $\mathrm{Nd}^{3+}$-complex-doped 6-FDA/UVR waveguides by a launched pump power of $25 \mathrm{~mW}$, and (b) $\mathrm{Al}_{2} \mathrm{O}_{3}: \mathrm{Nd}^{3+}$ waveguides by a launched pump power of $45 \mathrm{~mW}$.

\subsection{Energy-transfer Upconversion Parameters}

The internal net gain is reduced in heavily doped channel waveguides due to ETU processes among neighboring $\mathrm{Nd}^{3+}$ ions. To study the effect of ETU, three ETU processes originating in the metastable ${ }^{4} \mathrm{~F}_{3 / 2}$ level of the $\mathrm{Nd}^{3+}$ system were taken into account and expressed by a single macroscopic parameter $W_{\text {ETU }}$ in the simulation, as these processes lead to similar results concerning the population dynamics in the $\mathrm{Nd}^{3+}$ system.

From the simulation of $\mathrm{Nd}^{3+}$-complex-doped polymer and aluminum oxide channel waveguide amplifiers, the ETU parameter were determined for different $\mathrm{Nd}^{3+}$ concentrations at the three signal wavelengths independently. The experimental and simulated gains are in good agreement when fitting the ETU parameter in our waveguides, as discussed in previous sections. Figures 4.16(a) and (b) give the ETU parameters versus $\mathrm{Nd}^{3+}$ concentrations in the two types of waveguides. The ETU parameter of $\mathrm{Nd}^{3+}$ ions obtained in the two types of waveguides from the gain spectra shows a linear dependence on the ion concentration, and a linear fit was applied to the results at each wavelength.

For the ETU parameter determined by fitting the measured gain at 873 and $1064 \mathrm{~nm}$ in $\mathrm{Nd}^{3+}$-complex-doped 6-FDA/UVR (Fig. 4.16(a)), the linear fits at 873 and $1064 \mathrm{~nm}$ almost coincide, i.e., the ETU parameters obtained from the rate equations at 
the two wavelengths agree well. From the simulation of $\mathrm{Nd}^{3+}$-doped amplifiers at 873 $\mathrm{nm}$, ETU parameters of $0.065,0.118,0.163$, and $0.407 \times 10^{-16} \mathrm{~cm}^{3} \mathrm{~s}^{-1}$ were determined, while the ETU parameters determined at $1064 \mathrm{~nm}$ were $0.070,0.135,0.205$, and $0.383 \times 10^{-16} \mathrm{~cm}^{3} \mathrm{~s}^{-1}$, for $\mathrm{Nd}^{3+}$ concentrations of $0.30,0.60,1.03$, and $2.03 \times 10^{20} \mathrm{~cm}^{-3}$, respectively. Most of the values obtained at the two wavelengths at each $\mathrm{Nd}^{3+}$ concentration show less than $10 \%$ deviation, and a maximum deviation of $33 \%$ was observed for the $\mathrm{Nd}^{3+}$ concentration of $0.30 \times 10^{20} \mathrm{~cm}^{-3}$. The deviation is caused by experimental errors in the spectroscopic measurements and gain investigations.

The ETU parameters of $\mathrm{Nd}^{3+}$ obtained at the three wavelengths in $\mathrm{Al}_{2} \mathrm{O}_{3}$ at each $\mathrm{Nd}^{3+}$ concentration are given in Fig. 4.16 (b). For each concentration, the values fitted to the gain measured at the three different wavelengths show less than $10 \%$ deviation from each other; only for the $\mathrm{Nd}^{3+}$ concentration of $0.65 \times 10^{20} \mathrm{~cm}^{-3}$ a deviation of $14 \%$ was observed. In Section 3.5.2, the ETU parameters of $\mathrm{Al}_{2} \mathrm{O}_{3}: \mathrm{Nd}^{3+}$ have been studied by measuring luminescence decay curves and use of a nonexponential model. The ETU parameters determined from this method were $0.58,0.68$, 1.00 , and $2.20 \times 10^{-16} \mathrm{~cm}^{3} \mathrm{~s}^{-1}$ at $\mathrm{Nd}^{3+}$ concentrations of $0.65,1.13,1.68$, and $2.95 \times 10^{20}$ $\mathrm{cm}^{-3}$, respectively, which are given as the open dots in Fig. 4.16(b). The main fitting error of ETU parameters in this approach could be the deviation of the starting fitting point, due to the fluctuation of the excitation laser output. The deviation of the ETU parameters determined from two independent approaches is around $6.5-23 \%$ for different $\mathrm{Nd}^{3+}$ concentrations, which is still at a reasonable range considering the experimental and fitting errors introduced in both approaches. The agreement of the ETU parameters from the two independent approaches shows that the obtained ETU parameters are reliable.

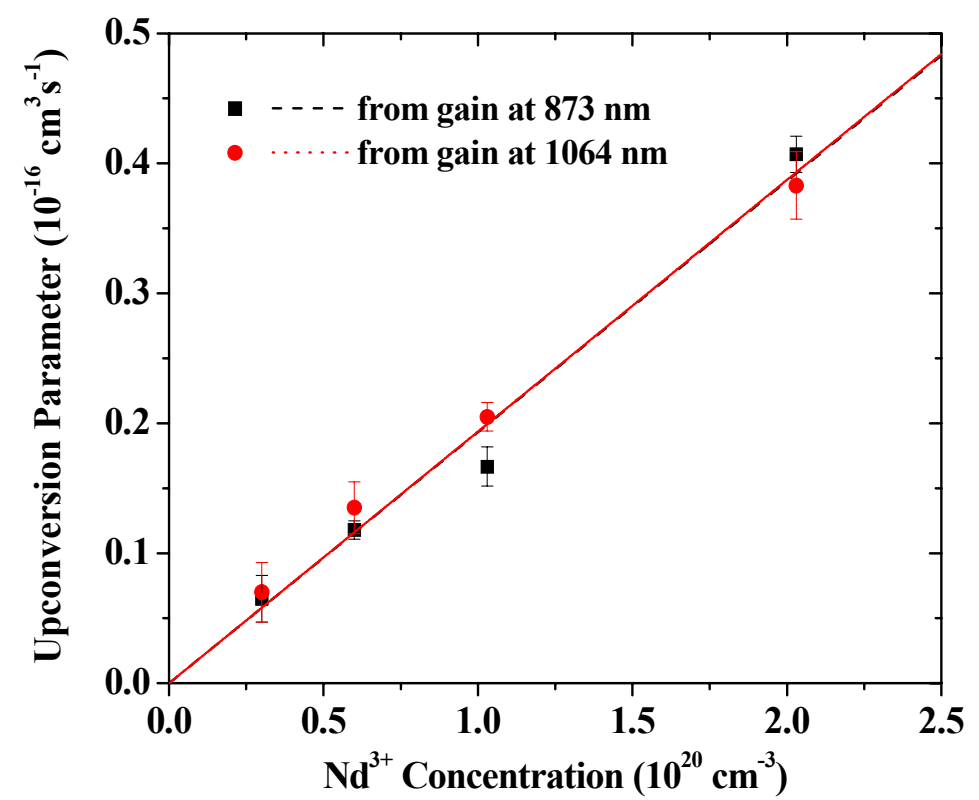

(a) 


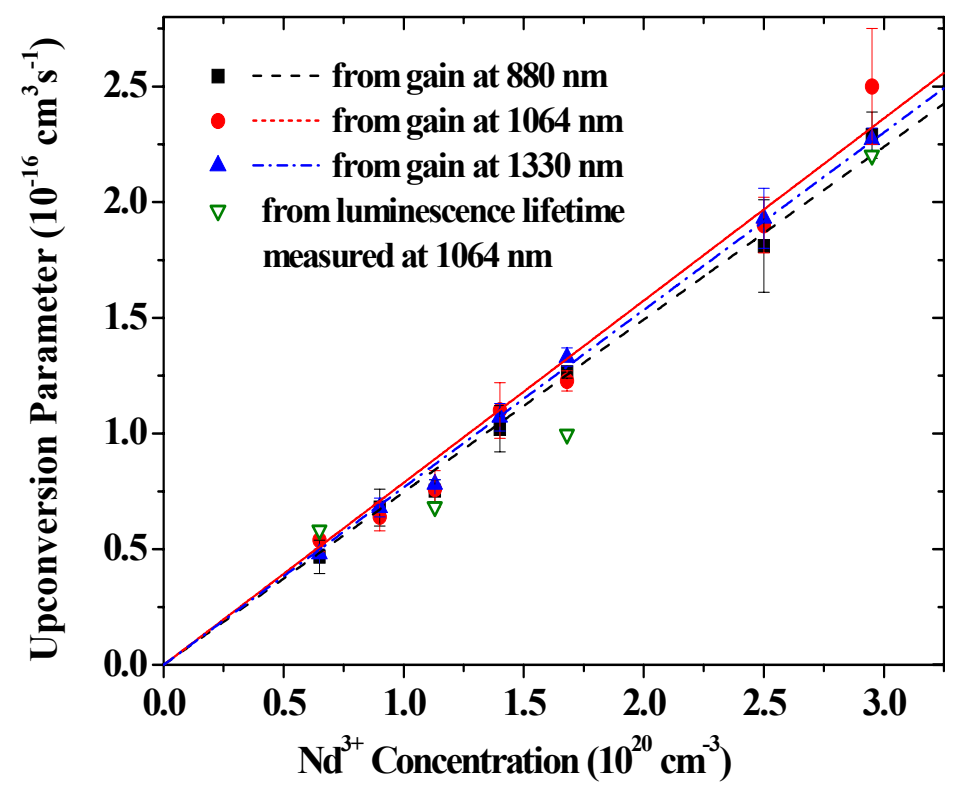

(b)

Fig. 4.16. ETU parameter as a function of $\mathrm{Nd}^{3+}$ concentration (a) fitted by the rate-equation model at 873 and $1064 \mathrm{~nm}$ in $\mathrm{Nd}^{3+}$-complex-doped 6-FDA/UVR waveguides, and (b) fitted by the rate-equation model at $880 \mathrm{~nm}, 1064 \mathrm{~nm}$, and $1330 \mathrm{~nm}$, and derived from luminescence lifetime measurements at $1064 \mathrm{~nm}$ in $\mathrm{Al}_{2} \mathrm{O}_{3}: \mathrm{Nd}^{3+}$ (see Fig. 3.28).

Assuming that the ETU parameter of $\mathrm{Nd}^{3+}$ increases linearly with ion concentration, we averaged the values obtained in each type of waveguide for each concentration. The ETU parameters obtained in this way from our study were compared to the values in different host materials from the literature. Figure 4.17 shows the averaged ETU parameters versus $\mathrm{Nd}^{3+}$ concentration in open dots, and a linear fitting line of these points for 6-FDA/UVR and $\mathrm{Al}_{2} \mathrm{O}_{3}$ waveguides. The ETU parameters of $\mathrm{Nd}^{3+}$ in various materials [88, 89, 125-129] are also given in the same figure and Table 4.3 for comparison.

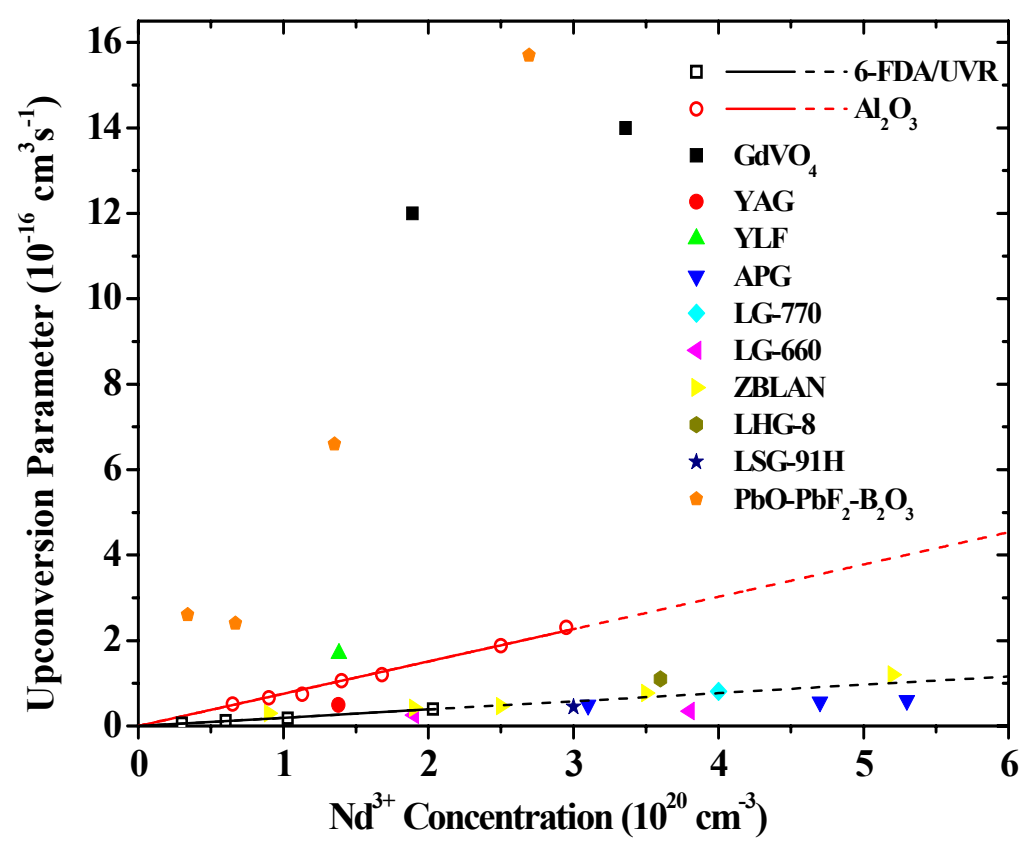

Fig. 4.17. ETU parameters of $\mathrm{Nd}^{3+}$ in different host materials. 
Table. 4.3. ETU parameters of $\mathrm{Nd}^{3+}$ in different host materials.

\begin{tabular}{|c|c|c|c|}
\hline Materials & $\begin{array}{l}\text { Upconversion parameter } \\
\left(\times 10^{-16} \mathrm{~cm}^{3} \mathrm{~s}^{-1}\right)\end{array}$ & $\begin{array}{c}\mathrm{Nd}^{3+} \text { concentrations } \\
\left(10^{20} \mathrm{~cm}^{-3}\right)\end{array}$ & Ref. \\
\hline Nd(TTA) ${ }_{3}$ phen-doped & 0.07 & 0.30 & This work \\
\hline \multirow[t]{3}{*}{ 6-FDA/UVR } & 0.13 & 0.60 & \\
\hline & 0.19 & 1.03 & \\
\hline & 0.40 & 2.03 & \\
\hline \multirow[t]{7}{*}{$\mathrm{Al}_{2} \mathrm{O}_{3}: \mathrm{Nd}^{3+}$} & 0.52 & 0.65 & This work \\
\hline & 0.67 & 0.90 & \\
\hline & 0.74 & 1.13 & \\
\hline & 1.06 & 1.40 & \\
\hline & 1.20 & 1.68 & \\
\hline & 1.88 & 2.50 & \\
\hline & 2.32 & 2.95 & \\
\hline \multirow[t]{2}{*}{$\mathrm{Nd}: \mathrm{GdVO}_{4}$} & 12 & 1.89 & {$[121]$} \\
\hline & 14 & 3.36 & \\
\hline Nd:YAG & 0.5 & 1.38 & {$[88,89]$} \\
\hline Nd:YLF & 1.7 & 1.383 & {$[88]$} \\
\hline Nd:APG & 0.49 & 3.1 & [122] \\
\hline \multirow[t]{2}{*}{ (phosphate glass) } & 0.56 & 4.7 & \\
\hline & 0.60 & 5.3 & \\
\hline $\begin{array}{l}\text { Nd:LG-770 } \\
\text { (phosphate glass) }\end{array}$ & 0.81 & 4.0 & {$[122]$} \\
\hline Nd:LG-660 & 0.26 & 1.9 & {$[122]$} \\
\hline (silicate glass) & 0.35 & 3.8 & \\
\hline Nd:ZBLAN & 0.46 & 2.5 & {$[122]$} \\
\hline $\begin{array}{l}\text { Nd:LHG-8 } \\
\text { (phosphate glass) }\end{array}$ & 1.15 & 3.6 & {$[123]$} \\
\hline \multicolumn{4}{|l|}{ (silicate glass) } \\
\hline Nd:lead fluoroborate glasses & 2.6 & 0.34 & {$[124]$} \\
\hline \multirow[t]{3}{*}{$\left(\mathrm{PbO}-\mathrm{PbF}_{2}-\mathrm{B}_{2} \mathrm{O}_{3}\right)$} & 2.4 & 0.67 & \\
\hline & 6.6 & 1.352 & \\
\hline & 15.7 & 2.695 & \\
\hline Nd:glass & 0.3 & $0.9 \times 10^{20} \mathrm{~cm}^{-3}$ & {$[125]$} \\
\hline \multirow[t]{3}{*}{$\left(\mathrm{ZrF}_{4}-\mathrm{BaF}_{2}-\mathrm{LaF}_{3}-\mathrm{AlF}_{3}-\mathrm{NaF}-\mathrm{XNdF}\right)$} & 0.42 & $1.9 \times 10^{20} \mathrm{~cm}^{-3}$ & \\
\hline & 0.77 & $3.5 \times 10^{20} \mathrm{~cm}^{-3}$ & \\
\hline & 1.2 & $5.2 \times 10^{20} \mathrm{~cm}^{-3}$ & \\
\hline
\end{tabular}

The obtained ETU parameters in 6-FDA/UVR are slightly smaller than values of ETU parameters in many other $\mathrm{Nd}^{3+}$-doped host materials, which might be due to the isolation of each $\mathrm{Nd}^{3+}$ ion by its organic ligands increasing the minimum distance and reduces the interaction between neighboring $\mathrm{Nd}^{3+}$ ions. $\mathrm{Al}_{2} \mathrm{O}_{3}: \mathrm{Nd}^{3+}$, on the other hand, exhibits a higher ETU parameter than the majority of glass, polymer, and crystalline materials, which might be an indication of a close distance between two $\mathrm{Nd}^{3+}$ ions in $\mathrm{Al}_{2} \mathrm{O}_{3}$. 


\subsection{Summary}

Small signal gain measurements have been applied to investigate the internal net gain of $\mathrm{Nd}^{3+}$-complex doped 6-FDA/UVR and $\mathrm{Al}_{2} \mathrm{O}_{3}: \mathrm{Nd}^{3+}$ channel waveguide amplifiers.

In $\mathrm{Nd}^{3+}$-complex-doped, photo-defined polymer channel waveguide amplifiers internal net optical gain has been demonstrated in the wavelength range $865-930 \mathrm{~nm}$, with up to $2.8 \mathrm{~dB}$ gain obtained at $873 \mathrm{~nm}$ by optimization of the $\mathrm{Nd}^{3+}$ concentration and channel waveguide length. Gain per unit length at $873 \mathrm{~nm}$ and 1064 $\mathrm{nm}$ of $2.0 \mathrm{~dB} / \mathrm{cm}$ and $5.7 \mathrm{~dB} / \mathrm{cm}$, respectively, has been measured.

In $\mathrm{Al}_{2} \mathrm{O}_{3}: \mathrm{Nd}^{3+}$ channel waveguide, a maximum gain of $14.4 \mathrm{~dB}$ and $5.1 \mathrm{~dB}$ has been observed at 1064 and $1330 \mathrm{~nm}$, respectively. Optical gain has been demonstrated in the wavelength range $865-930 \mathrm{~nm}$, with up to $3.0 \mathrm{~dB}$ gain obtained at $880 \mathrm{~nm}$ by optimization of the $\mathrm{Nd}^{3+}$ concentration and channel waveguide length. Gain per unit length at $880 \mathrm{~nm}, 1064 \mathrm{~nm}$, and $1330 \mathrm{~nm}$ of $1.57 \mathrm{~dB} / \mathrm{cm}, 6.30 \mathrm{~dB} / \mathrm{cm}$, and $1.93 \mathrm{~dB} / \mathrm{cm}$, respectively, has been measured.

The calculated and measured gain spectra on the ground-state transition show good agreement with measured data in both polymer and aluminium oxide waveguides. With the gain simulated by a rate-equation model as well as fitting of measured luminescence decay curves, the ETU parameter of $\mathrm{Nd}^{3+}$ in the two hosts has been studied.

Such high-gain waveguide amplifiers may be well suited for providing optical gain in integrated optical applications, e.g. loss-less data transmission in optical interconnects and telecommunication, signal enhancement in integrated Raman spectroscopy, as well as potential lasing. The wavelength range of optical gain in such amplifiers can be extended by doping with other rare-earth ions.

The applications of these waveguide amplifiers will be discussed in the next two chapters. 
Chapter 4 


\section{Chapter 5}

\section{$\mathrm{Nd}^{3+}$-doped Polymer Waveguide Lasers}

The laser performance of $\mathrm{Nd}^{3+}$-complex-doped polymer channel waveguides was investigated using a continuous-wave excitation at $800 \mathrm{~nm}$. CW laser operation was achieved near $1060.2 \mathrm{~nm}$ and $878 \mathrm{~nm}$ in $\mathrm{Nd}^{3+}$-complex-doped polymer channel waveguides with output powers of up to $0.98 \mathrm{~mW}$ and 0.20 $m W$, respectively. The stability of the laser output was examined with $C W$ excitation. To the best of our knowledge, this is the first report of a rare-earthion-doped polymer waveguide laser as well as a $C W$ solid-state polymer laser. 


\subsection{Introduction}

Lasers have been reported in many optically pumped solid-state waveguides based on dye-doped polymers [55] and highly luminescent semiconducting polymers [56]. However, no CW lasing has been achieved in polymers due to the nature of the semiconductor and dye polymer lasers (described in Section 1.3.2). Although laser emission from rare-earth-ion-doped polymer systems was demonstrated in bulk polymer materials $[58,59]$ and a range of solid-state waveguide lasers based on a variety of $\mathrm{Nd}^{3+}$-doped dielectric materials have been reported [29, 126-129], a rareearth-ion-doped continuous polymer waveguide laser has as yet not been demonstrated.

The waveguide laser has a great application potential, e.g. for use as a miniaturized integrated light source for lab-on-chip applications involving infrared and quantum-dot labeling in life sciences and medical diagnostics, or as a tool for fluorescence correlation spectroscopy. $\mathrm{Nd}^{3+}$ is arguably the most important active rare-earth ion for the demonstration of laser operation, especially the laser operation of its four-level transition ${ }^{4} \mathrm{~F}_{3 / 2} \rightarrow{ }^{4} \mathrm{I}_{11 / 2}$ at approximately $1060 \mathrm{~nm}$, due to the large emission cross-section resulting in a very low laser threshold.

In the previous Chapter, $\mathrm{Nd}^{3+}$-complex-doped polymer waveguide amplifiers with high gains at transitions ${ }^{4} \mathrm{~F}_{3 / 2} \rightarrow{ }^{4} \mathrm{I}_{11 / 2}$ and ${ }^{4} \mathrm{~F}_{3 / 2} \rightarrow{ }^{4} \mathrm{I}_{9 / 2}$ have been demonstrated. In this chapter, $\mathrm{CW}$ laser operation of $\mathrm{Nd}^{3+}$-complex-doped 6-FDA/UVR channel waveguides near $880 \mathrm{~nm}$ and $1060 \mathrm{~nm}$ will be reported. To the best of our knowledge, this work represents simultaneously the first demonstration of a rare-earth-ion-doped polymer waveguide laser and a CW solid-state polymer laser.

This work was performed in collaboration with Dr. Christos Grivas from the University of Southampton, UK, who joined our research group for a sabbatical visit in 2009.

\subsection{Laser Experiment}

Laser performance was investigated in $\mathrm{Nd}^{3+}$-complex-doped polymer channel waveguide for the optimal $\mathrm{Nd}^{3+}$ concentration of $1.03 \times 10^{20} \mathrm{~cm}^{-3}$ determined from gain measurement in previous Chapter.

Laser tests of the $\mathrm{Nd}^{3+}$-doped 6-FDA/UVR channel waveguides around 880 and $1060 \mathrm{~nm}$ were performed at room temperature by longitudinally pumping at 800 $\mathrm{nm}$ with a CW Ti:Sapphire laser (see Fig. 5.1). The laser cavity was formed by attaching lightweight thin dielectric mirrors to the polished end-faces of the waveguide using the surface tension of a small amount of fluorinated liquid (Fluorinert, FC-70) for adhesion. The mirrors used for incoupling were high-reflective $(\mathrm{R}=99.8 \%)$ at the lasing wavelength and had a transmission of $94 \%$ at the pump wavelength. A mirror with high reflectivity and a set of mirrors with different reflectivities at the laser wavelengths of $880 \mathrm{~nm}$ and $1060 \mathrm{~nm}$, respectively, were used as output couplers. The microscope objectives used for in- and outcoupling had a magnification of $\times 4$ and $\times 10$, respectively. The pump laser beam was expanded using a telescope consisting of two spherical lenses with focal lengths of $f=-40$ and 200 $\mathrm{mm}$, respectively, in order to fill the aperture of the incoupling objective. The outcoupled waveguide mode, after passing through a RG1000 or RG850 filter (for 1060-nm or 878-nm lasing, respectively) to block the residual transmitted pump light, 
was directed onto a power meter or a spectrometer as required. The coupling efficiency was optimized by adjusting the position of the waveguide and tuning the pump laser to find the lowest threshold power at the onset of lasing or by imaging the laser mode on a CCD camera.

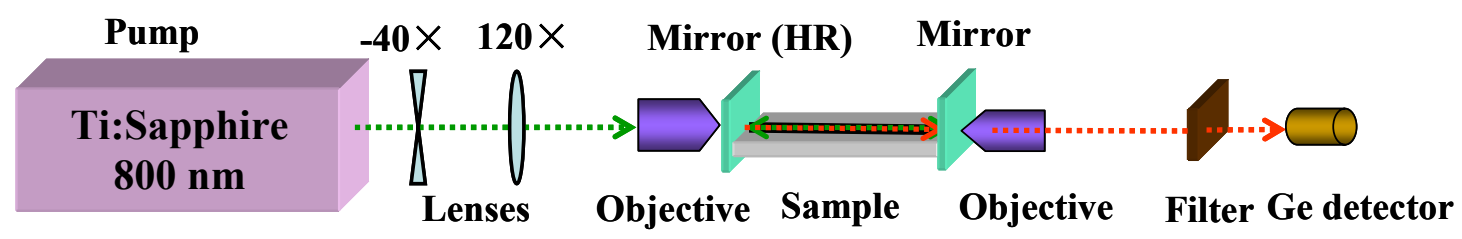

Fig. 5.1. Laser experiment using butt-coupled mirrors.

\subsection{Laser Results}

Laser oscillation was obtained for all the channels investigated at a wavelength near $1060 \mathrm{~nm}$, which corresponds to the most intense peak of the waveguide luminescence spectrum. For a 16-mm-long cavity formed by two high reflective mirrors a laser threshold of $84 \mathrm{~mW}$ absorbed pump power was obtained. Figure 5.2(a) shows damped relaxation oscillations obtained via modulation of the pump laser at an absorbed pump power of $100 \mathrm{~mW}$.

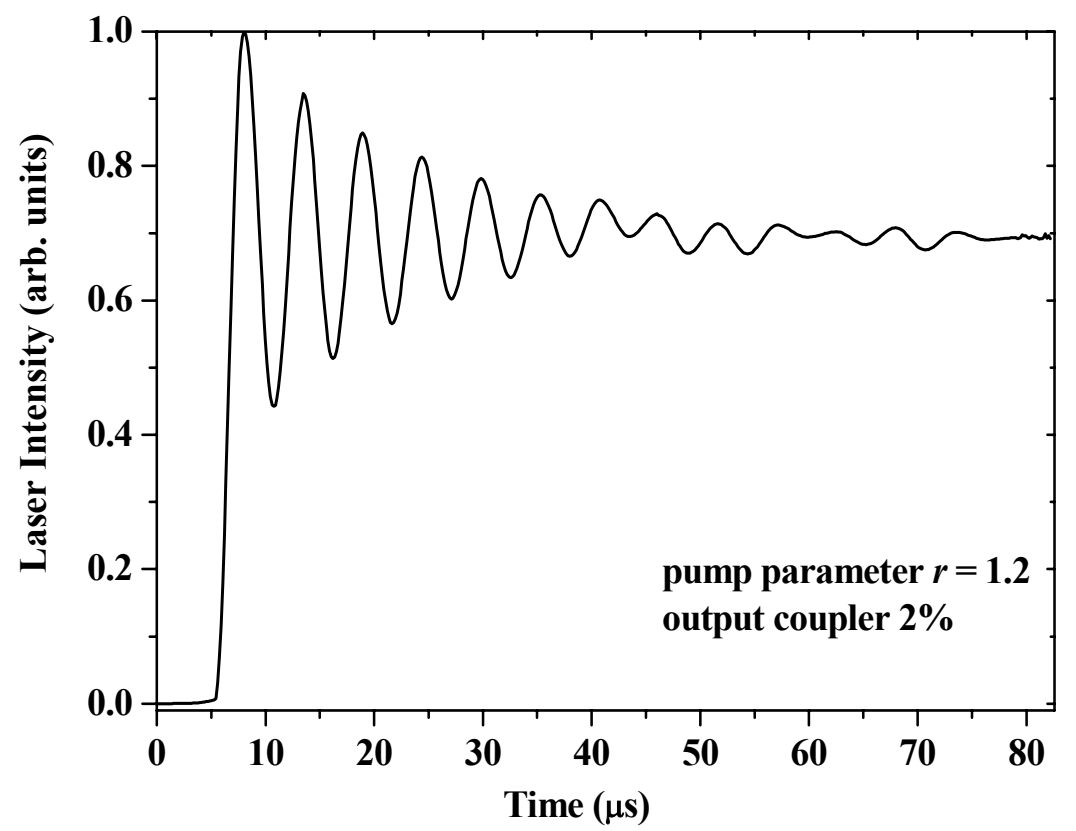

(a) 


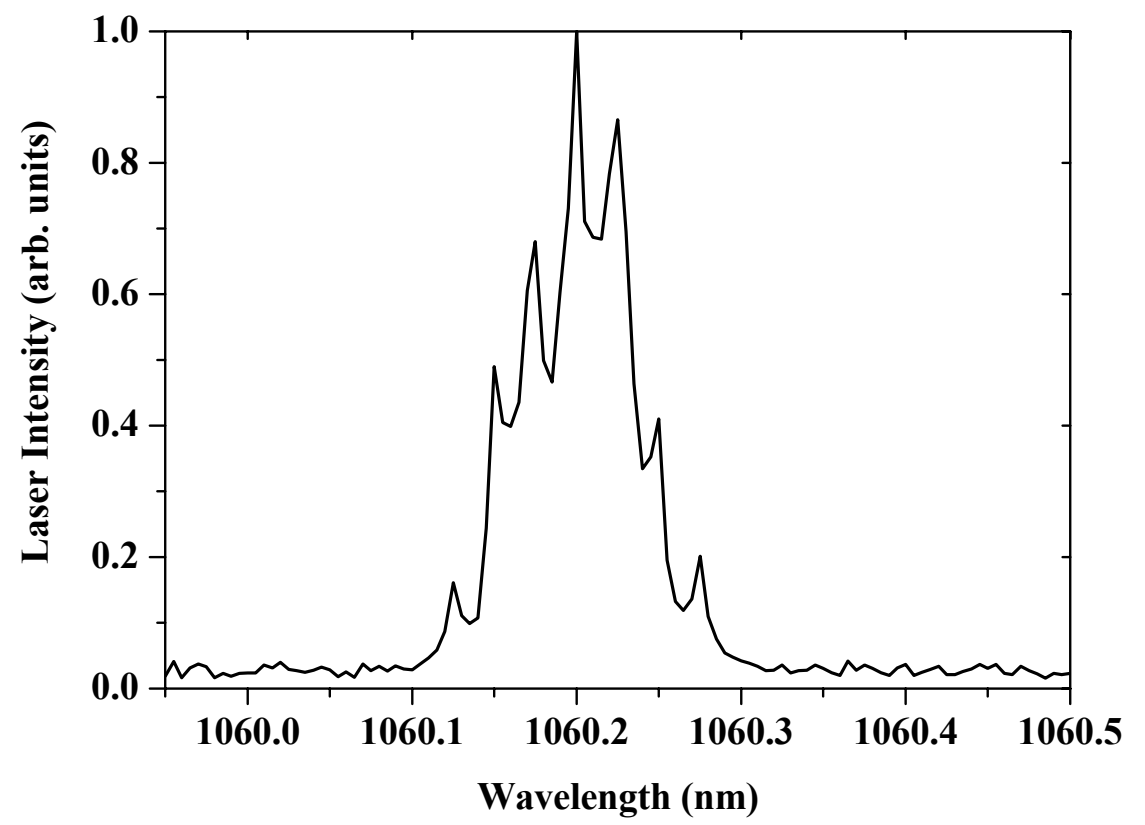

(b)

Fig. 5.2. Characterization of laser emission from a $\mathrm{Nd}^{3+}$-doped 6-FDA/UVR channel waveguide at an absorbed pump power of $100 \mathrm{~mW}$ corresponding to a pump rate $r=1.2$ : (a) relaxation oscillations and (b) typical laser emission spectrum.

Figure 5.2(b) shows a laser spectrum obtained for an absorbed pump power just above the laser threshold. The separation of the peaks observed in the spectrum, which correspond to the longitudinal cavity modes, can be calculated for the cavity length $l=16 \mathrm{~mm}$ using the equation:

$$
\Delta \lambda=\frac{\lambda^{2}}{2 \cdot l \cdot n}
$$

The calculated peak separation is $0.023 \mathrm{~nm}$, which fits well with the value of 0.025 $\mathrm{nm}$ observed from the measured laser spectrum.

By replacing the high reflectivity outcoupling mirror with one having a transmission of $2 \%$ at the lasing wavelength the threshold value increased to $101 \mathrm{~mW}$. Figure 5.3 shows the laser output power as a function of absorbed pump power. The maximum output power was $440 \mu \mathrm{W}$ at $1060 \mathrm{~nm}$ for $148 \mathrm{~mW}$ of absorbed pump power and a slope efficiency $\eta$ of $0.95 \%$ with respect to absorbed pump power was derived. 


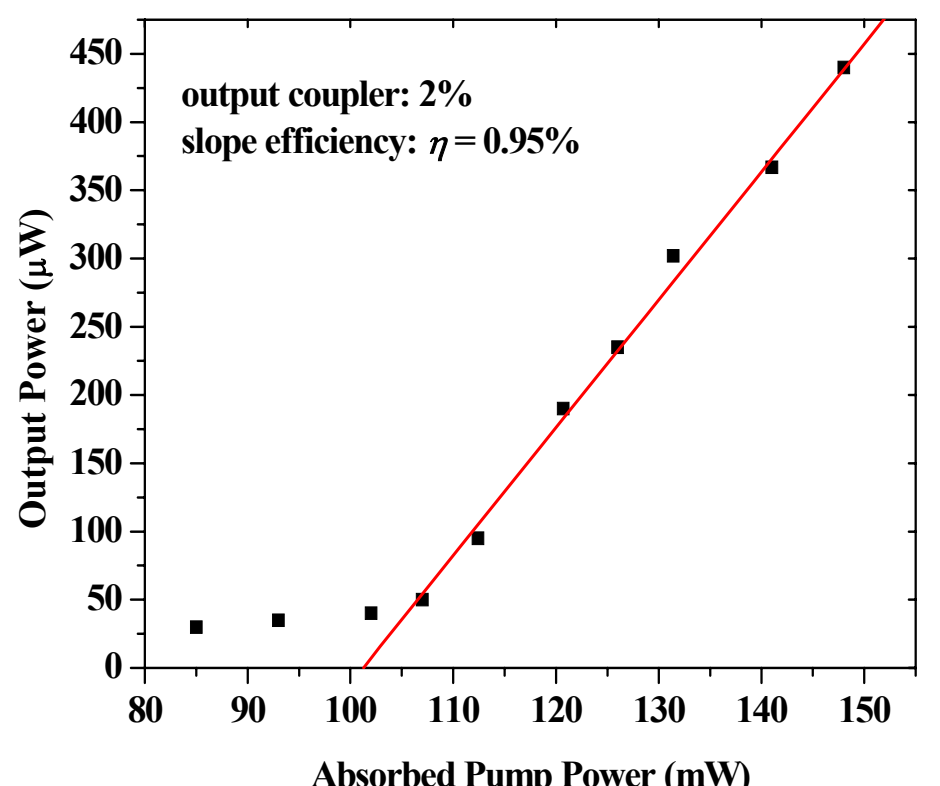

Fig. 5.3. Laser output power in a 16-mm-long $\mathrm{Nd}^{3+}$-complex-doped 6-FDA/UVR channel waveguide as a function of absorbed pump power for the four-level transition at $1060.2 \mathrm{~nm}$.

The laser performance at $1060 \mathrm{~nm}$ was further studied in a shorter channel waveguide sample of length of $7.5 \mathrm{~mm}$, using successively a set of four outcoupling mirrors with transmission values of $1.8 \%, 3 \%, 4 \%$, and $5 \%$ at the laser wavelength. Figure 5.4 shows the laser spectrum at high pump power. The output versus input power characteristics obtained for each of them is shown in Fig. 5.5. The highest slope efficiency $\eta$ of $2.15 \%$ and the maximum output power of $0.98 \mathrm{~mW}$ for $129 \mathrm{~mW}$ of absorbed power were obtained using a 5\% outcoupling mirror. Lasing could not be achieved using the outcoupling mirror with the next highest transmission available ( $T$ $=10 \%$ ), as the pump intensities required were destructive for the channels.

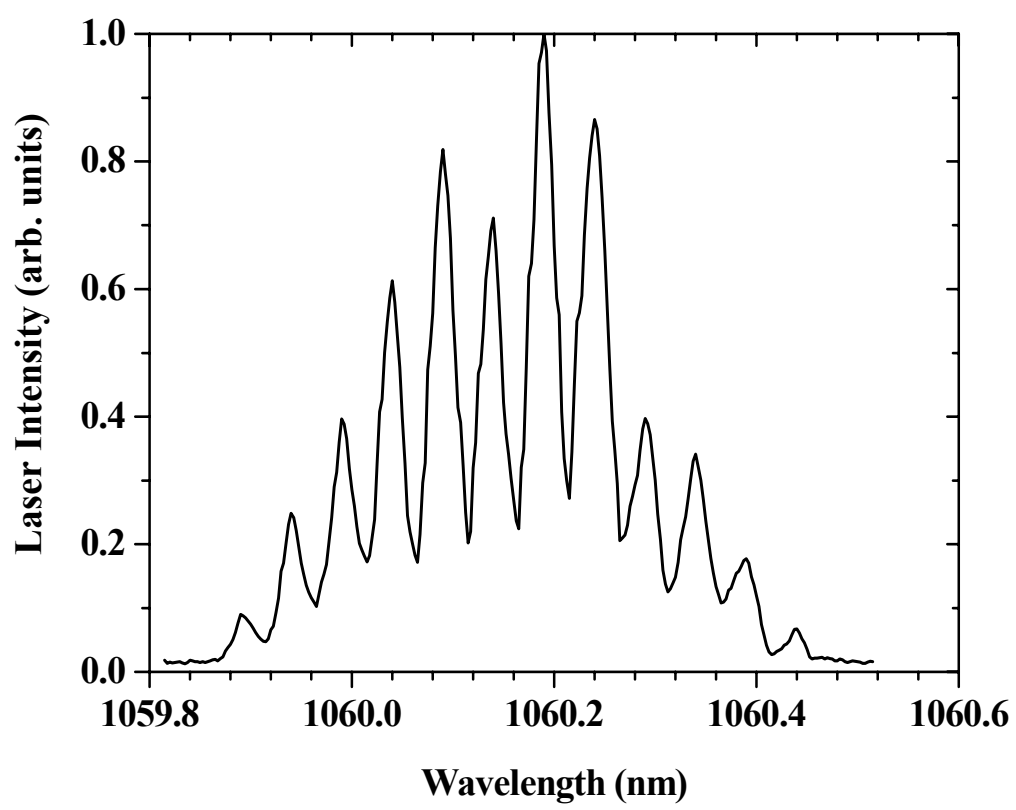

Fig. 5.4. Typical laser emission spectrum at $1060.2 \mathrm{~nm}$ in a $7.5-\mathrm{mm}$-long $\mathrm{Nd}^{3+}$-complex-doped 6-FDA/UVR channel waveguide at high pump power. 


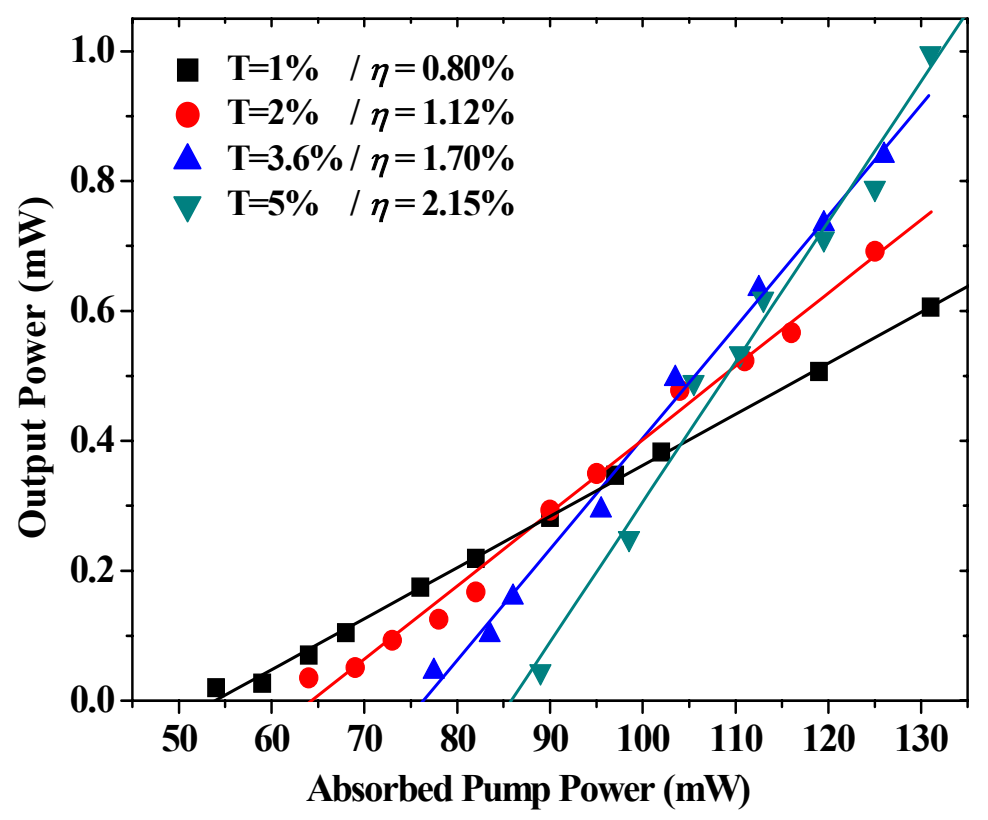

Fig.5.5. Laser output power in a 7.5-mm-long $\mathrm{Nd}^{3+}$-complex-doped 6-FDA/UVR channel waveguide as a function of absorbed pump power for the four-level transition at $1060.2 \mathrm{~nm}$ using a set of outcoupling mirrors.

A result that exceeded our expectations was the laser operation on the quasithree-level transition near $878 \mathrm{~nm}$. Re-absorption losses combined with the relatively low stimulated-emission cross section at this wavelength $\left(\sigma_{e} \sim 1.2 \times 10^{-20} \mathrm{~cm}^{2}\right.$ as compared to $\sigma_{e} \sim 4.4 \times 10^{-20} \mathrm{~cm}^{2}$ for the dominant four-level transition), require high pump powers, which however counter-poses the need to maintain the pump power at a low level to avoid detrimental effects on laser performance induced by thermal degradation of the material. Using two HR mirrors as input and output couplers lasing was observed at an absorbed pump power threshold of $67 \mathrm{~mW}$ (Fig. 5.6). Figure 5.7 shows the laser output as a function of absorbed pump power for $2.2 \%$ outcoupling, which yields a slope efficiency of $0.35 \%$ and a laser threshold of $74.5 \mathrm{~mW}$. 


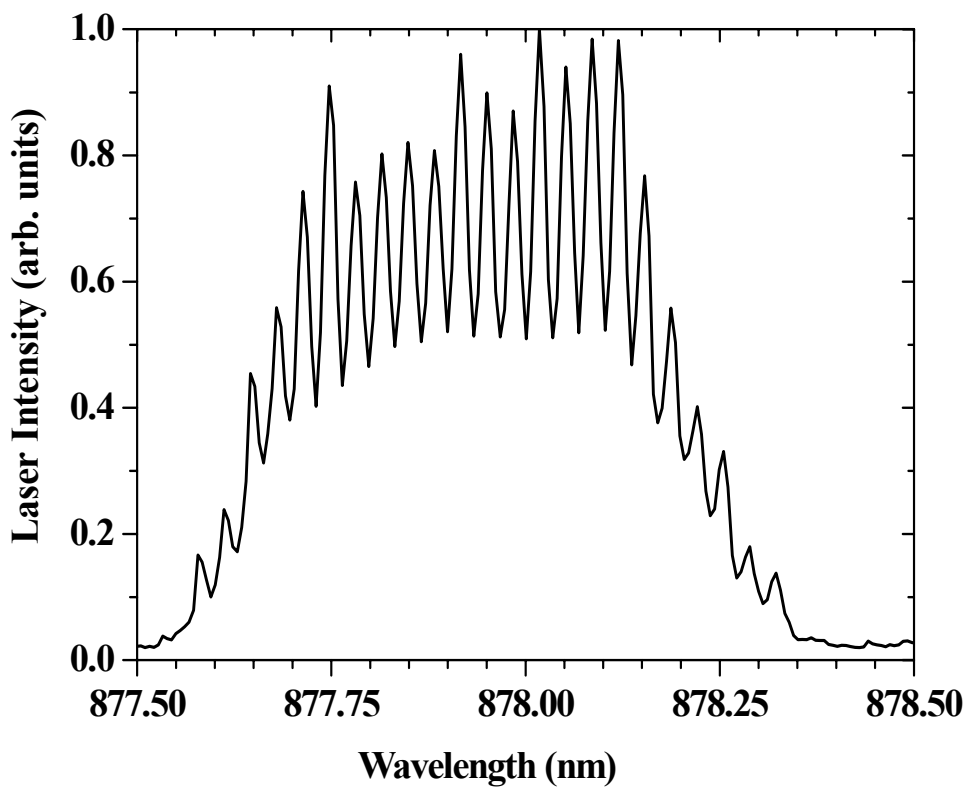

Fig. 5.6. Typical laser emission spectrum at $878 \mathrm{~nm}$ in a 7.5-mm-long $\mathrm{Nd}^{3+}$-complex-doped 6FDA/UVR channel waveguide at high pump power.

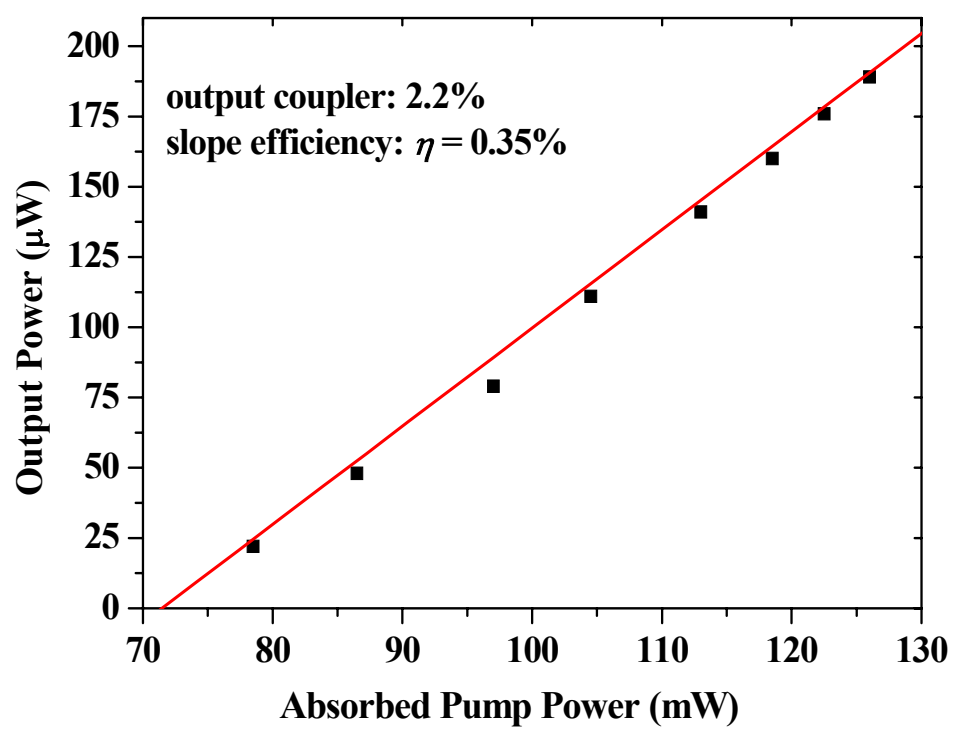

Fig.5.7. Laser output power in a 7.5-mm-long $\mathrm{Nd}^{3+}$-complex-doped 6-FDA/UVR channel waveguide as a function of absorbed pump power for the three-level transition at $878 \mathrm{~nm}$.

An upper limit for the propagation loss in the waveguide at $1060 \mathrm{~nm}$ was obtained using the Findlay-Clay method [130]. The latter is applicable to four-level laser systems showing negligible depopulation of the ground state, for which the absorbed power threshold $P_{t h}$ is dependent on the outcoupling level and is given by

$$
P_{t h}=K \cdot\left[\left(2 \cdot \mathrm{a} \cdot l-\ln \left(R_{1} R_{2}\right)\right],\right.
$$


where $a$ is the propagation loss coefficient in $\mathrm{cm}^{-1}, R_{1}$ and $R_{2}$ are the intensity reflectivities of the in- and out-coupling mirrors, respectively, and $K$ is a constant dependent on the mode sizes of the laser and pump beams and material properties of the gain medium [131]. By inserting in Eq. 5.2 the values for $P_{t h}$ obtained for each of the out-coupling mirrors used and then plotting $P_{t h} / 2 \cdot l$ as a function of $-\ln \left(R_{1} \cdot R_{2}\right) / 2 \cdot l$, a straight line is obtained (Fig. 5.8). Its intercept with the abscissa yields a value of $0.032 \mathrm{~cm}^{-1}$ for $a$. By assuming that it is only due to propagation loss in the waveguide (in reality it also accounts for losses due to imperfections in the attachment of the cavity mirrors), it corresponds to an upper propagation loss value of $0.14 \mathrm{~dB} / \mathrm{cm}$. While the uncertainty in the derivation of loss is rather high as a result of the low outcoupling used, the value derived is in good agreement with the propagation loss measured using the cut-back method $(0.1 \mathrm{~dB} / \mathrm{cm})$.

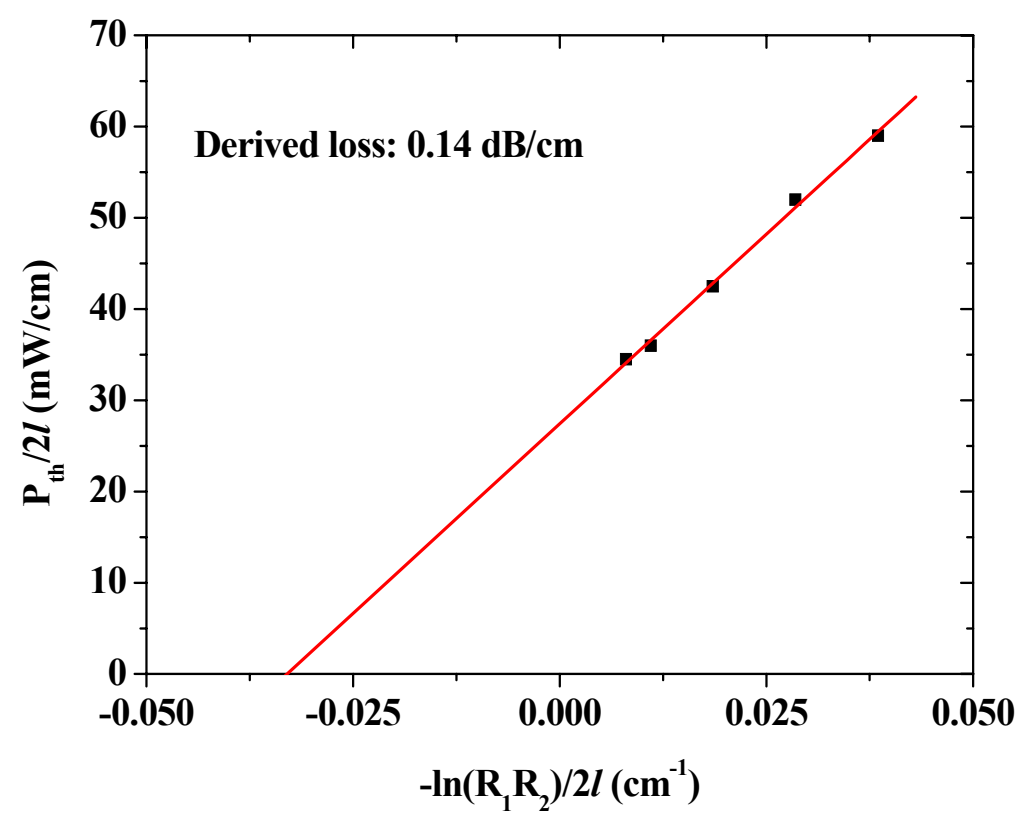

Fig. 5.8. Plot of $P_{t h} / 2 l$ as a function of $-\ln \left(R_{1} \cdot R_{2}\right) / 2 \cdot l$. The intercept with the abscissa provides the propagation attenuation coefficient $\alpha$.

To study the consistency of the laser performance with the propagation loss derived, an estimate of the slope efficiency $\eta$ was made using a model, which describes the effects of transverse mode profiles on $\eta$ in longitudinally pumped lasers [132]. According to this theory $\eta$ is given by the expression

$$
\eta=\left(\frac{\lambda_{P}}{\lambda_{L}}\right) \cdot\left(\frac{-\ln \left(R_{2}\right)}{-\ln \left(R_{1} \cdot R_{2}\right)+2 \cdot l \cdot \alpha}\right) \cdot \eta_{q} \cdot \eta_{o}
$$

where $\lambda_{L}$ and $\lambda_{P}$ are the laser and pump wavelengths, $\eta_{q}$ is the pump quantum efficiency, and $\eta_{\mathrm{o}}$ is the effective overlap of the pump with the laser mode in the waveguide. Assuming unity for $\eta_{o}$ and $\eta_{q}$, and $R_{1}=0.998$ and $R_{2}=0.99$ for the in- and outcoupling mirrors, respectively, we obtain from Eq. 4.7 a maximum attainable value of $12.2 \%$ for $\eta$, for the specific outcoupling level. This value is significantly higher 
than the experimental one $(\eta=0.8 \%)$ for reasons that may include a quantum efficiency substantially lower than unity, a lower than unity overlap factor $\eta_{\mathrm{o}}$ due to the multimode nature of the waveguide, and thermal effects in the active medium, which are more pronounced than in crystalline waveguides.

An important aspect of the operation of optically pumped polymer lasers is their lifetime stability, which can be adversely affected by material degradation or even damage induced by the temperature increase in the active medium during pumping. The onset of such effects also determines the thermal limit for power scaling in polymer lasers. To date, the maximum reported values of lifetime stability of organic semiconductor and dye-doped lasers operating in the pulsed regime range from $10^{6}$ to $10^{7}$ pulses and from $10^{5}$ to $10^{6}$ pulses, respectively [56].

One of the main concerns in optically pumped organic solid-state lasers is the material degradation or even damage induced by photo-oxidation effects and heating. Photo-oxidation is related to the creation of defects such as $\mathrm{C}=\mathrm{O}, \mathrm{COOH}$, and $\mathrm{COH}$ in the pumping process. To investigate the occurrence of any such effects during pumping optical choppers operating with various duty cycles ranging from $8 \%$ to $50 \%$ were inserted to the pump beam prior to its coupling into the individual waveguides. However, no difference was detected between the $\mathrm{CW}$ and these different quasi-CW states of laser operation in terms of performance.

The output stability of our polymer waveguide lasers was studied as a function of operation time for three different absorbed pump power settings. In Fig. 5.9 , it is shown conclusively that for $100 \mathrm{~mW}$ of absorbed pump power the active medium exhibits a level of thermal stability that is appropriate for long-term operation of at least 2 hours without any signs of deterioration of the laser performance. Stable $\mathrm{CW}$ lasing behavior is observed up to an absorbed pump power of $130 \mathrm{~mW}$. However, for an absorbed pump power of $150 \mathrm{~mW}$, the output power decreases significantly from an initial level of $\sim 1 \mathrm{~mW}$ attained after the onset of pumping and stabilizes to a value of $\sim 0.2 \mathrm{~mW}$ after $\sim 20 \mathrm{~s}$. This behaviour is attributed to material degradation via photo-oxidation (i.e. creation of carbonyl defects in the active medium, which quench the luminescence), leading to optical loss at the pump and signal wavelengths. At absorbed powers of $200 \mathrm{~mW}$, a sharp decrease in the output power is observed immediately after the onset of lasing followed by its abrupt termination, which indicates that the temperature rise could not be sustained by the material, leading to the destruction of the channel. 


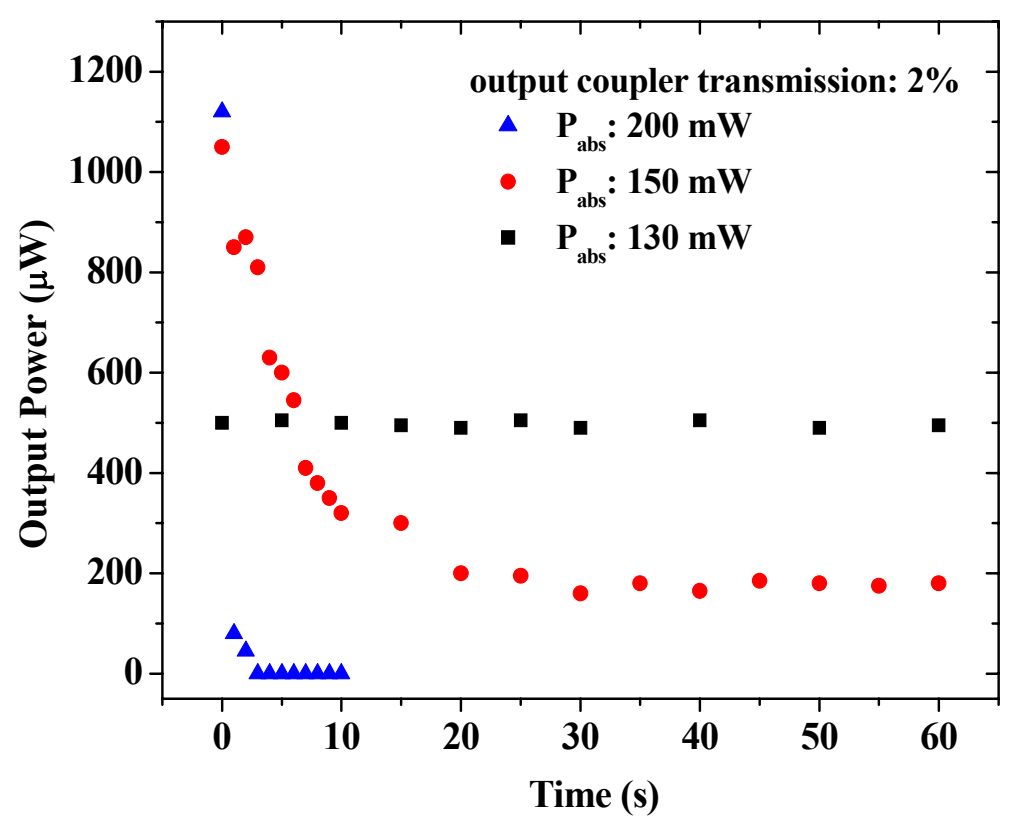

(a)

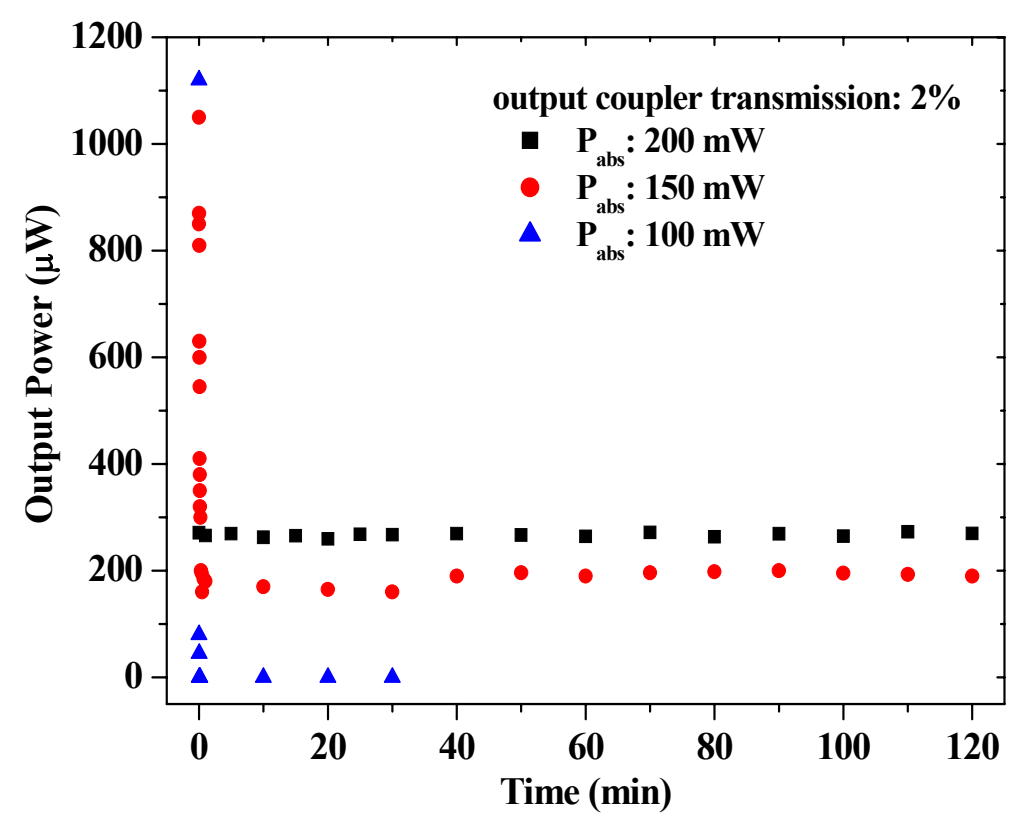

(b)

Fig. 5.9. Laser output power as a function of operation time for different absorbed pump power regimes for a period of (a) 1 minute and (b) 2 hours.

\subsection{Summary}

$\mathrm{CW}$ laser oscillation near $1060.2 \mathrm{~nm}$ was demonstrated in $\mathrm{Nd}^{3+}$-complex-doped polymer channel waveguides with output powers of up to $0.98 \mathrm{~mW}$, for pump powers low enough to allow stable laser emission for at least 2 hours.

Moreover, laser operation with output power up to $0.20 \mathrm{~mW}$ was also achieved on the quasi-three-level transition near $878.0 \mathrm{~nm}$. 
To the best of our knowledge, this is the first report of a rare-earth-ion-doped polymer waveguide laser as well as a CW solid-state polymer laser.

The miniature laser developed here is useful as an integrated light source for lab-on-a-chip applications involving infrared dye and quantum-dot labeling, or as a tool for fluorescence correlation spectroscopy. 
Chapter 5 


\section{Chapter 6}

\section{$\mathrm{Nd}^{3+}$-doped Waveguide Amplifiers Integrated in Optical Backplanes}

In this Chapter the feasibility of using $\mathrm{Al}_{2} \mathrm{O}_{3}: \mathrm{Nd}^{3+}$ channel waveguides as amplifiers in optical backplanes is discussed. Large-core $\mathrm{Al}_{2} \mathrm{O}_{3}: \mathrm{Nd}^{3+}$ channel waveguide amplifiers were fabricated and characterized. Upon optimization, a maximum gain of $2.03 \mathrm{~dB}$ was measured in 3-cm-long, 3.0- $\mu \mathrm{m}$-high, and 2.5 - $\mu$ m-wide channel waveguides with a $\mathrm{Nd}^{3+}$ concentration of $0.91 \times 10^{20} \mathrm{~cm}^{-3}$, for a launched pump power of $55 \mathrm{~mW}$. Polymer waveguides used in optical backplanes were provided by IBM Zurich. $\mathrm{Al}_{2} \mathrm{O}_{3}: \mathrm{Nd}^{3+}$ channel waveguide samples were applied in between two such polymer waveguide samples to demonstrate the feasibility of optical amplification in optical backplanes. The coupling loss between the two types of waveguides was investigated in order to optimize their channel geometries. A tapered waveguide was designed to improve the pump intensity in the active waveguide region. A maximum 0.42 $d B$ internal net gain has been demonstrated in a combination of a polymer waveguide coupled to an $\mathrm{Al}_{2} \mathrm{O}_{3}: \mathrm{Nd}^{3+}$ waveguide. Thereby, a solution for compensating optical loss in optical interconnection is provided. 


\subsection{Introduction}

The development of optical interconnects in printed circuit boards (PCBs) [2-4] is driven by the increasing bandwidth requirements in servers, supercomputers, and switch routers. Optical transmission via waveguides offers the potential of a much larger capacity than electrical data transmission through copper lines. Recently, a 12channel card-to-card optical interconnect link with embedded polymer waveguides and optical signal generation by a diode laser operating at $850 \mathrm{~nm}$ with data transmission up to $10 \mathrm{~Gb} / \mathrm{s}$ per channel has been reported by Dellmann et al. from IBM Zurich [3]. The wavelength of $850 \mathrm{~nm}$ was chosen due to the maturity of VCSEL technology at this wavelength.

Investigations on the optical power budget for realistic polymer-waveguidebased high-speed links via optical backplanes indicate that the optical power in boards is reduced significantly by the coupling and routing dependent loss as well as waveguide propagation loss [1]. Therefore, additional signal amplification within the board is necessary to compensate optical losses arising due to waveguide materials, signal routing, and input/output coupling. Integration of amplifying materials into optical backplanes is an efficient solution to maintain sufficient optical power level. The amplification device can be mounted on optical backplanes by various techniques, such as flip-flop [1,132] and flip-chip [1,133]. Figure 6.1 shows a schematic picture of a waveguide amplifier integrated laterally into optical backplanes via flip-flop technique.

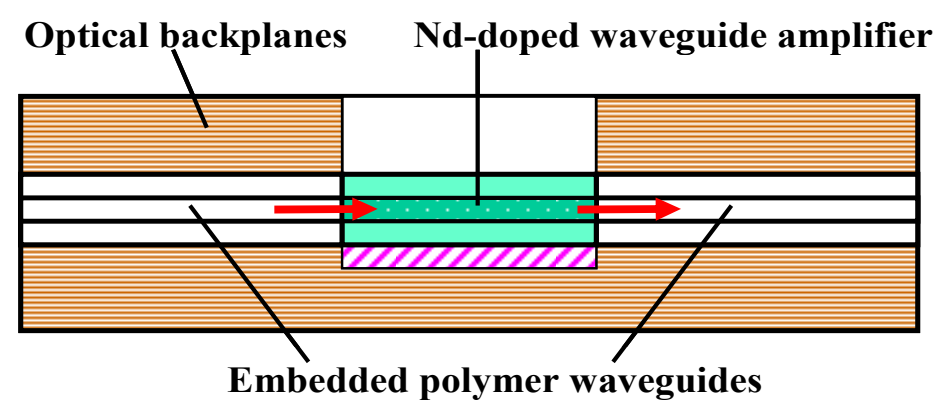

Fig. 6.1. Schematics of an optical waveguide amplifier integrated into optical backplanes by flip-flop technique.

In the previous Chapter, $\mathrm{Nd}^{3+}$-doped waveguide amplifiers with broadband gain extending from $865-930 \mathrm{~nm}$, a wavelength range which is close to the wavelength of $850 \mathrm{~nm}$ currently used in optical backplanes, have been demonstrated. Such amplifiers, although not matching exactly the currently available cheap VCSEL wavelengths, can be of interest for signal amplification in future optical interconnections to compensate the propagation loss arising from waveguide materials, light coupling and signal routing.

$\mathrm{Nd}^{3+}$-complex-doped 6-FDA/UVR waveguides are promising for such an application because of the compatibility with the polymer waveguides in optical backplanes, and can be directly applied in optical backplanes. However, the major disadvantage of this material is the low damage threshold at high pump intensity and short lifetime. 
$\mathrm{Al}_{2} \mathrm{O}_{3}$ waveguides have high thermal stability and are suitable as host materials for rare-earth-ion-doped high-speed amplifiers. $\mathrm{Al}_{2} \mathrm{O}_{3}: \mathrm{Nd}^{3+}$ waveguides with high gain are highly interesting for signal amplification in future optical interconnections. A peak gain of $3 \mathrm{~dB}$ at $880 \mathrm{~nm}$ in $\mathrm{Al}_{2} \mathrm{O}_{3}: \mathrm{Nd}^{3+}$ amplifiers has been achieved in single-mode small-core waveguides. Large geometrical cross sections are favorable for the envisaged application, because the currently developed optical backplanes consist of transverse multi-mode waveguides. Therefore, in order to greatly facilitate alignment for efficient signal in- and out-coupling, the amplifier design must be carried out accordingly. On the other hand, in order to achieve sufficient gain for optical amplification, waveguide geometries must be small in order to obtain a high pump intensity. Therefore, optimized waveguide geometries are necessary for this type of amplification and integration with polymer waveguides for applications in optical backplanes. Low-loss polymer waveguides embedded in optical backplanes have been fabricated and provided by IBM Zurich. $\mathrm{Al}_{2} \mathrm{O}_{3}: \mathrm{Nd}^{3+}$ waveguides were applied in between to amplify a propagating optical signal.

In this Chapter the polymer channel waveguides used to embed in optical backplanes provided by IBM Zurich are introduced. The large-core $\mathrm{Al}_{2} \mathrm{O}_{3}: \mathrm{Nd}^{3+}$ channel waveguide amplifiers have been characterized and tested in combination with the polymer waveguides. A study towards minimization of optical losses arising from waveguide coupling was performed and a waveguide taper in $\mathrm{Al}_{2} \mathrm{O}_{3}$ was designed. Finally, internal net gain in the system was demonstrated.

\subsection{Polymer Waveguides Embedded in Optical Backplanes}

Low-loss polymer waveguides embedded in PCB backplanes have been successfully demonstrated by IBM Zurich [3]. The polysiloxane-based polymers [134] have been fabricated by IBM Zurich, and in this work we have combined them with our $\mathrm{Al}_{2} \mathrm{O}_{3}$ waveguides for the demonstration of optical amplification in optical backplanes.

Flame Retardant 4 (FR4), a glass reinforced epoxy laminate which is commonly used for making printed circuit boards was used as the substrate. A lower cladding layer was deposited on the FR4 substrate using a doctor blading technique [135]. The core layer was deposited using spin-coating, and channel waveguides were realized using mask lithography and laser direct writing. The waveguides have slight trapezoidal cross-section, and the sidewall angle varies with the thickness and width of the channel. The sidewall inclination can be reduced using a higher effective writing speed. The refractive indices of the waveguide core and cladding at $850 \mathrm{~nm}$ are 1.515 and 1.479 , respectively.

Polymer channel waveguides up to $50 \times 50 \mu \mathrm{m}^{2}$ have been fabricated by such a process. The minimum thickness of the core layer that can be achieved by spincoating is around $5 \sim 6 \mu \mathrm{m}$, while the smallest channel width is about $5 \sim 6 \mu \mathrm{m}$. Figure 6.2 shows microscope images of channel waveguides with different cross sections. The unpolarized propagation loss at $880 \mathrm{~nm}$ measured in a $6 \times 6-\mu \mathrm{m}^{2}$ multimode channel waveguide by the cut-back method was $0.34 \pm 0.09 \mathrm{~dB} / \mathrm{cm}$. 


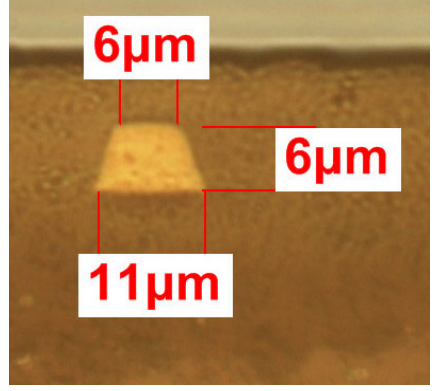

(a)

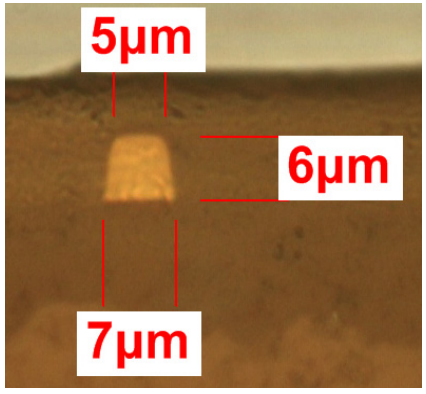

(b)

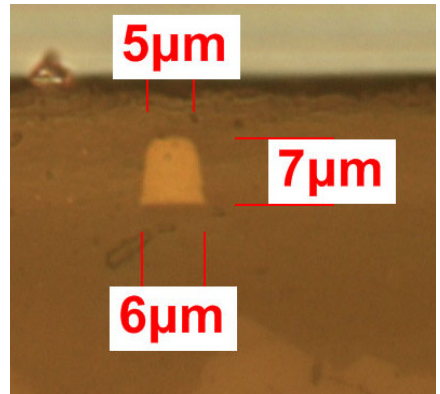

(c)

Fig. 6.2. Microscope images of polymer channel waveguides with cross sections of (a) $6 \times 6 \mu \mathrm{m}^{2}$, (b) $5 \times 6 \mu \mathrm{m}^{2}$, (c) $5 \times 7 \mu \mathrm{m}^{2}$.

Such polymer waveguides were used for the demonstration of optical amplification in optical backplanes by directly placing an $\mathrm{Al}_{2} \mathrm{O}_{3}: \mathrm{Nd}^{3+}$ waveguide sample between two polymer waveguides sections. Considering the thickness limitation of $\mathrm{Al}_{2} \mathrm{O}_{3}$ layers, the thickness and width of polymer channel waveguides used for the final demonstration was 6-7 $\mu \mathrm{m}$ and 6-12 $\mu \mathrm{m}$, respectively. The PhoeniX software package employing the finite difference (FD) method was used to study the mode properties. Table 6.1 shows the results of the mode calculation in polymer waveguides with different cross sections for TE polarization. Similar results were achieved for TM polarization.

Table. 6.1. Calculated confinement factor $\Gamma$ of the fundamental mode and Gaussian beam waists of polymer waveguides for TE polarization.

\begin{tabular}{|c|c|c|c|c|c|}
\hline \multirow{2}{*}{$\begin{array}{l}\text { Width } \\
(\mu \mathrm{m})\end{array}$} & \multirow{2}{*}{$\begin{array}{l}\text { Thickness } \\
\qquad(\mu \mathrm{m})\end{array}$} & \multirow{2}{*}{$\begin{array}{l}\text { Side wall } \\
\text { angle }\end{array}$} & \multirow{2}{*}{$\begin{array}{l}\text { Confinement factor } \Gamma \\
\text { of fundamental mode }\end{array}$} & \multicolumn{2}{|c|}{ Gaussian Beam Waist $(\mu \mathrm{m})$} \\
\hline & & & & Horizontal $w_{x}$ & Vertical $w_{y}$ \\
\hline 5 & 6 & $\sim 80.5^{\circ}$ & 0.978 & 2.447 & 2.450 \\
\hline 6 & 6 & $\sim 67.4^{\circ}$ & 0.962 & 3.264 & 2.441 \\
\hline 5 & 7 & $\sim 85.9^{\circ}$ & 0.987 & 2.273 & 2.801 \\
\hline
\end{tabular}

\section{3 $\mathrm{Al}_{2} \mathrm{O}_{3}: \mathrm{Nd}^{3+}$ Large-core Waveguide Amplifiers}

\subsubsection{Motivation and Waveguide Geometries}

In Chapter 3, the fabrication of $\mathrm{Al}_{2} \mathrm{O}_{3}: \mathrm{Nd}^{3+}$ waveguides with and without cladding layers have been described (Section 3.3.2), and small-geometry single-mode waveguide amplifiers without cladding have been used for the characterization of optical gain at the three relevant transitions of $\mathrm{Nd}^{3+}$. In order to test the performance of waveguide amplifiers in optical backplanes, the $\mathrm{Al}_{2} \mathrm{O}_{3}: \mathrm{Nd}^{3+}$ waveguides were designed to be directly coupled between two polymer waveguides. This approach aimed at demonstrating the feasibility of the concept and not as final device geometry.

Figure 6.3 shows the geometry of the $\mathrm{Al}_{2} \mathrm{O}_{3}$ waveguides used in this study, which is similar to that discussed in previous chapters. In order to match the size of the polymer waveguides used in optical backplanes (Section 6.2), large-core $\mathrm{Al}_{2} \mathrm{O}_{3}: \mathrm{Nd}^{3+}$ channel waveguides up to $5 \mu \mathrm{m}$ thick and $12 \mu \mathrm{m}$ wide were fabricated. In order to achieve a good confinement in the horizontal direction of the waveguide, etch 
depths of $0.5-1.0 \mu \mathrm{m}$ were realized. To match the mode confinement in polymer waveguides, a PECVD $\mathrm{SiO}_{2}$ layer with a refractive index of 1.452 at $633 \mathrm{~nm}$ and a thickness of $5 \mu \mathrm{m}$ was deposited on top of the $\mathrm{Al}_{2} \mathrm{O}_{3}: \mathrm{Nd}^{3+}$ ridge waveguides as the cladding layer. The waveguides were tested without planarization of the top of the PECVD $\mathrm{SiO}_{2}$ layer, as it did not affect the guiding behavior.

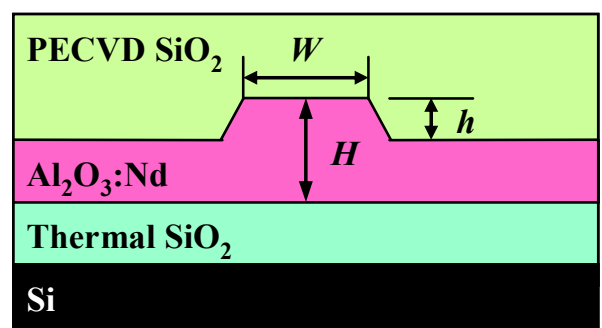

Fig. 6.3. Cross section of a large-core $\mathrm{Al}_{2} \mathrm{O}_{3}: \mathrm{Nd}^{3+}$ waveguide with $\mathrm{PECVD} \mathrm{SiO}_{2}$ cladding.

In order to study the mode properties of these large-core $\mathrm{Al}_{2} \mathrm{O}_{3}: \mathrm{Nd}^{3+}$ waveguides, the PhoeniX software package was used. Optical modes in channel waveguides with various thicknesses $H$ and widths $W$ at pump and signal wavelengths of $800 \mathrm{~nm}$ and $880 \mathrm{~nm}$, respectively, were investigated. $\mathrm{Al}_{2} \mathrm{O}_{3}: \mathrm{Nd}^{3+}$ waveguides with various thicknesses were studied, and the results are presented in Table 6.2.

Table 6.2. Number of modes, confinement factor $\Gamma$ of the fundamental mode, and Gaussian beam waists of $\mathrm{Al}_{2} \mathrm{O}_{3}: \mathrm{Nd}^{3+}$ waveguides from mode calculations for TE polarization: (a) thickness: $H=1 \mu \mathrm{m}$, etch depth: $h=0.5 \mu \mathrm{m}$; (b) thickness: $H=3 \mu \mathrm{m}$, etch depth: $h=1.0 \mu \mathrm{m}$; (c) thickness: $H=5 \mu \mathrm{m}$, etch depth: $h=1.0 \mu \mathrm{m}$.

(a)

\begin{tabular}{cccccc}
\hline \hline $\begin{array}{c}\text { Width } \\
\boldsymbol{W}(\boldsymbol{\mu m})\end{array}$ & $\begin{array}{c}\text { Wavelength } \\
(\mathbf{n m})\end{array}$ & $\begin{array}{c}\text { Number } \\
\text { of Modes }\end{array}$ & $\begin{array}{c}\text { Confinement factor } \boldsymbol{\Gamma} \text { of } \\
\text { fundamental mode }\end{array}$ & \multicolumn{2}{c}{ Gaussian Beam Waist $(\boldsymbol{\mu m})$} \\
Horizontal $\boldsymbol{w}_{\boldsymbol{x}}$ & Vertical $\boldsymbol{w}_{\boldsymbol{y}}$ \\
\hline \multirow{2}{*}{1} & 800 & 1 & 0.937 & 0.764 & 0.489 \\
& 880 & 1 & 0.923 & 0.803 & 0.508 \\
\hline \multirow{2}{*}{2} & 800 & 2 & 0.957 & 1.014 & 0.496 \\
& 880 & 2 & 0.947 & 1.038 & 0.515 \\
\hline \multirow{2}{*}{5} & 800 & 5 & 0.963 & 2.013 & 0.496 \\
& 880 & 4 & 0.954 & 2.030 & 0.515 \\
\hline \multirow{2}{*}{10} & 800 & 12 & 0.963 & 3.756 & 0.496 \\
& 880 & 10 & 0.955 & 3.770 & 0.515 \\
\hline
\end{tabular}

\section{(b)}

\begin{tabular}{cccccc}
\hline \hline $\begin{array}{c}\text { Width } \\
\boldsymbol{W}(\boldsymbol{\mu m})\end{array}$ & $\begin{array}{c}\text { Wavelength } \\
(\mathbf{n m})\end{array}$ & $\begin{array}{c}\text { Number } \\
\text { of Modes }\end{array}$ & $\begin{array}{c}\text { Confinement factor } \boldsymbol{\Gamma} \text { of } \\
\text { fundamental mode }\end{array}$ & \multicolumn{2}{c}{$\begin{array}{c}\text { Gaussian Beam Waist }(\boldsymbol{\mu m}) \\
\text { Horizontal } \boldsymbol{w}_{\boldsymbol{x}}\end{array}$} \\
\hline \multirow{2}{*}{1.5} & 800 & 1 & 0.994 & 3.086 & 0.958 \\
& 880 & 1 & 0.992 & 3.161 & 0.971 \\
\hline \multirow{2}{*}{2} & 800 & 1 & 0.994 & 2.452 & 1.040 \\
& 880 & 1 & 0.993 & 2.513 & 1.052 \\
\hline \multirow{2}{*}{2.5} & 800 & 1 & 0.995 & 2.231 & 1.095 \\
& 800 & 1 & 0.994 & 2.281 & 1.107 \\
\hline 3 & 800 & 2 & 0.996 & 2.173 & 1.130
\end{tabular}




\begin{tabular}{cccccc} 
& 880 & 1 & 0.995 & 2.215 & 1.142 \\
\hline \multirow{2}{*}{5} & 800 & 3 & 0.997 & 2.570 & 1.169 \\
& 880 & 3 & 0.996 & 2.600 & 1.182 \\
\hline \multirow{2}{*}{10} & 800 & 4 & 0.998 & 4.209 & 1.177 \\
& 880 & 4 & 0.997 & 4.231 & 1.191 \\
\hline
\end{tabular}

(c)

\begin{tabular}{cccccc}
\hline \hline $\begin{array}{c}\text { Width } \\
\boldsymbol{W}(\boldsymbol{\mu m})\end{array}$ & $\begin{array}{c}\text { Wavelength } \\
(\mathbf{n m})\end{array}$ & $\begin{array}{c}\text { Number } \\
\text { of Modes }\end{array}$ & $\begin{array}{c}\text { Confinement factor } \boldsymbol{\Gamma} \text { of } \\
\text { fundamental mode }\end{array}$ & $\begin{array}{c}\text { Gaussian Beam Waist }(\boldsymbol{\mu m}) \\
\text { Horizontal } \boldsymbol{w}_{\boldsymbol{x}}\end{array}$ & \begin{tabular}{c} 
Vertical $\boldsymbol{w}_{\boldsymbol{y}}$ \\
\hline \multirow{2}{*}{2}
\end{tabular} \\
\hline 800 & 1 & 0.996 & 10.186 & 1.571 \\
& 880 & 1 & 0.995 & 10.271 & 1.585 \\
\hline \multirow{2}{*}{3} & 800 & 1 & 0.998 & 7.739 & 1.628 \\
& 880 & 1 & 0.998 & 7.841 & 1.640 \\
\hline \multirow{2}{*}{5} & 800 & 2 & 0.999 & 5.396 & 1.755 \\
& 880 & 2 & 0.999 & 5.464 & 1.766 \\
\hline \multirow{2}{*}{10} & 800 & 3 & 0.999 & 5.641 & 1.855 \\
& 880 & 3 & 0.999 & 5.680 & 1.868 \\
\hline
\end{tabular}

The maximum etch depth of $\mathrm{Al}_{2} \mathrm{O}_{3}: \mathrm{Nd}^{3+}$ waveguides that can be achieved by RIE is $\sim 1 \mu \mathrm{m}$. From the simulation results of Table 6.1 , the vertical confinement of the modes was mainly dependent on the thickness of the waveguide, and changed slightly by varying the channel width in each sample. The horizontal confinement of channel waveguides was strongly dependent on the etch depth into the $\mathrm{Al}_{2} \mathrm{O}_{3}: \mathrm{Nd}^{3+}$ layers and the channel width. In $1-\mu \mathrm{m}$-thick waveguides, confinement in the horizontal direction was improved by decreasing the channel width. However, in thick samples, the horizontal confinement in narrow channels decreased and modes were guided in the slab. In a 3- $\mu$ m-thick channel waveguide sample, the Gaussian beam waist of the fundamental mode in the horizontal direction decreases when reducing the width of the channel from $10 \mu \mathrm{m}$ to $3 \mu \mathrm{m}$. However, further reduction of the channel width from $3 \mu \mathrm{m}$ to $1 \mu \mathrm{m}$ results in a horizontal increase of the Gaussian beam waist from $2.22 \mu \mathrm{m}$ to $3.16 \mu \mathrm{m}$ at $880 \mathrm{~nm}$. Waveguides with channel width below $3 \mu \mathrm{m}$ and thinner than $3 \mu \mathrm{m}$ were single mode. The percentage of light guided in the slab waveguide region increases in waveguides of thickness less than $3 \mu \mathrm{m}$. The situation was even worse in 5 - $\mu$ m-thick waveguides due to the limited etch depth. Since reasonable confinement of modes in waveguides is required for acceptable pump intensities, the gain investigation was mainly focused on waveguides with thickness of $1 \mu \mathrm{m}$ and $3 \mu \mathrm{m}$.

The propagation losses measured by the cut-back method in a $3-\mu \mathrm{m}$-thick and $2-\mu \mathrm{m}$-wide channel waveguide sample were $\sim 0.60 \pm 0.07 \mathrm{~dB} / \mathrm{cm}$ and $\sim 0.53 \pm$ $0.08 \mathrm{~dB} / \mathrm{cm}$ for TE polarization at $800 \mathrm{~nm}$ and $880 \mathrm{~nm}$, respectively.

\subsubsection{Investigation of Waveguide Amplifiers}

The internal net gain of large-core $\mathrm{Al}_{2} \mathrm{O}_{3}: \mathrm{Nd}^{3+}$ channel waveguide amplifiers at the luminescence peak $(880 \mathrm{~nm})$ of the ground-state transition was experimentally investigated by small signal gain measurements using the pump probe method. The measured results were compared with the theoretical results calculated using the rateequation model, which has been explained in Chapter 2 and used for gain simulation 
in Chapter 4. Same spectroscopic parameters as given in Table 4.1, as well as the averaged ETU parameters of $\mathrm{Nd}^{3+}$ in $\mathrm{Al}_{2} \mathrm{O}_{3}$ determined in Chapter 4 (see Fig. 4.17) were used in the gain simulation. Channel waveguide samples with two different thicknesses of $1 \mu \mathrm{m}$ and $3 \mu \mathrm{m}$ were studied. The waveguide width was varied to optimize the gain.

Figure 6.4 shows the measured and simulated gain in a 1- $\mu \mathrm{m}$-thick layer with an etch depth of $0.5 \mu \mathrm{m}$ at a launched pump power of $45 \mathrm{~mW}$. The $\mathrm{Nd}^{3+}$ concentration in the waveguides is $0.5 \times 10^{20} \mathrm{~cm}^{3}$. The optical gain decreases dramatically when increasing the waveguide geometry at the same launched pump power, mainly due to the reduction of mode confinement and thus pump intensity in larger waveguide geometries. A maximum gain of $3.7 \mathrm{~dB}$ has been observed in a 6$\mathrm{cm}$-long sample for a channel width of $2 \mu \mathrm{m}$.

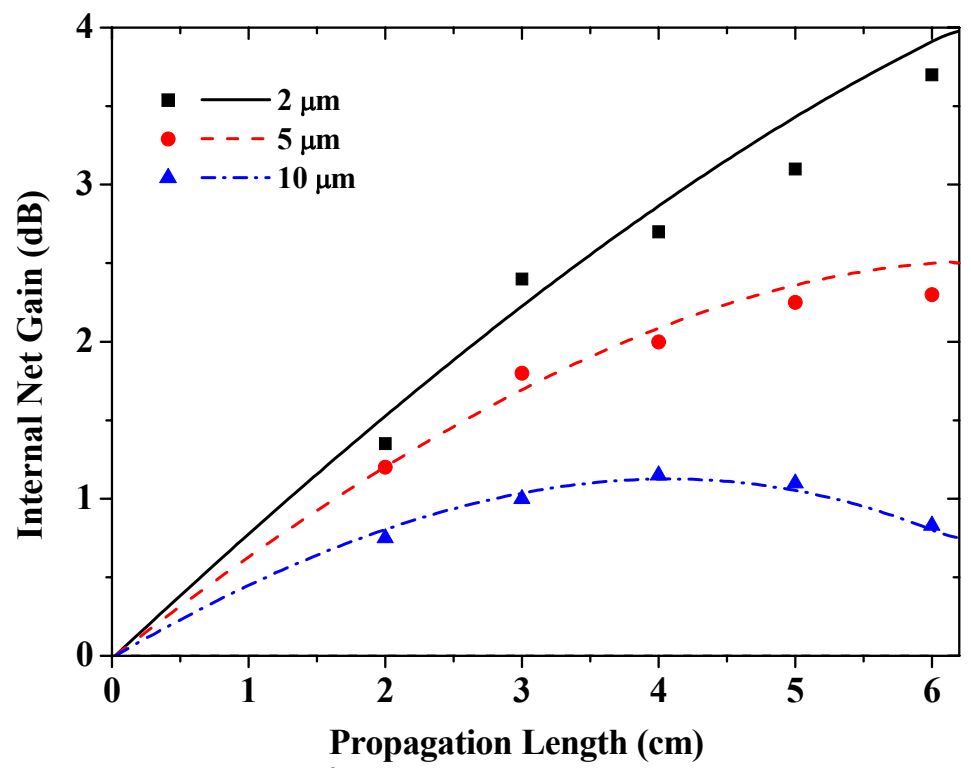

Fig. 6.4. Internal net gain of $\mathrm{Al}_{2} \mathrm{O}_{3}: \mathrm{Nd}^{3+}$ channel waveguides at $880 \mathrm{~nm}$ with a thickness of $1 \mu \mathrm{m}$, etch depth of $0.5 \mu \mathrm{m}$, and different channel widths of $2 \mu \mathrm{m}, 5 \mu \mathrm{m}$, and $10 \mu \mathrm{m}$, at a launched pump power of $45 \mathrm{~mW}$.

Optical gain has also been investigated in channel waveguides with a layer thickness of $3 \mu \mathrm{m}$ and etch depth of $1 \mu \mathrm{m}$. Figures 6.5 (a) and (b) show the measured and simulated gain in two samples with $\mathrm{Nd}^{3+}$ concentrations of 0.38 and $0.91 \times 10^{20}$ $\mathrm{cm}^{-3}$, at a launched pump power of $55 \mathrm{~mW}$. A maximum gain of $2.03 \mathrm{~dB}$ was observed in a 3-cm-long, 2.5- $\mu$ m-wide channel with a $\mathrm{Nd}^{3+}$ concentration of $0.91 \times 10^{20} \mathrm{~cm}^{-3}$. More gain can be achieved by further increasing the pump power and optimization of the propagation length. 


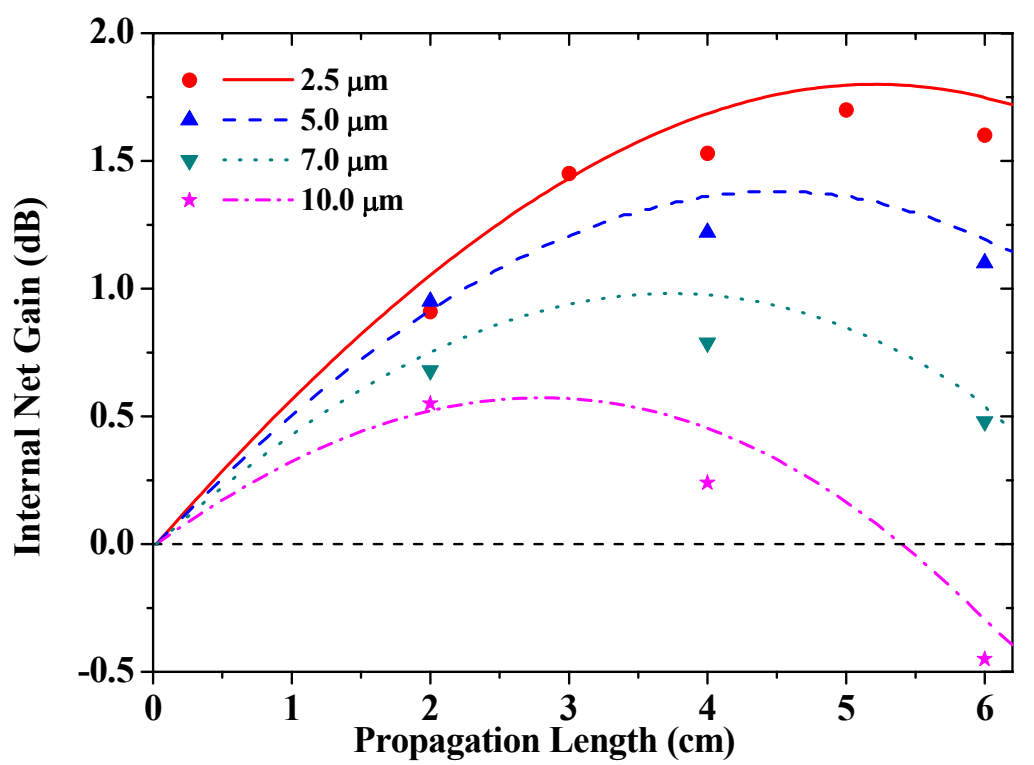

(a)

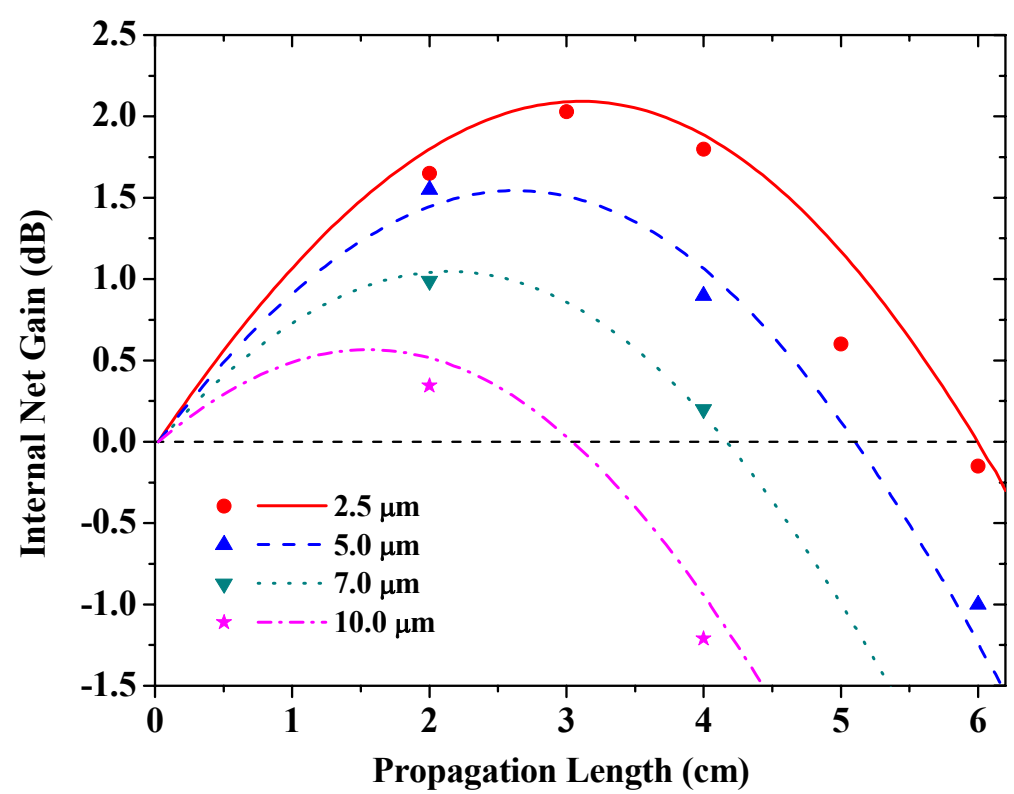

(b)

Fig. 6.5. Internal net gain of $\mathrm{Al}_{2} \mathrm{O}_{3}: \mathrm{Nd}^{3+}$ channel waveguides at $880 \mathrm{~nm}$ with a $\mathrm{Nd}^{3+}$ concentration of (a) $0.38 \times 10^{20} \mathrm{~cm}^{3}$ and (b) $0.91 \times 10^{20} \mathrm{~cm}^{3}$ in samples with a thickness of $3 \mu \mathrm{m}$ and etch depth of $1 \mu \mathrm{m}$, at a launched pump power of $55 \mathrm{~mW}$.

\subsection{Design of $\mathrm{Nd}^{3+}$-doped Waveguide Amplifiers Integrated in Optical Backplanes}

\subsection{1 $\mathrm{Al}_{2} \mathrm{O}_{3}: \mathrm{Nd}^{3+}$ Waveguides Integrated with Polymer Waveguides}

In this work, we focused on direct butt coupling of $\mathrm{Al}_{2} \mathrm{O}_{3}: \mathrm{Nd}^{3+}$ waveguides with polymer waveguides, with the goal to achieve net internal gain. Figure 6.6 shows a

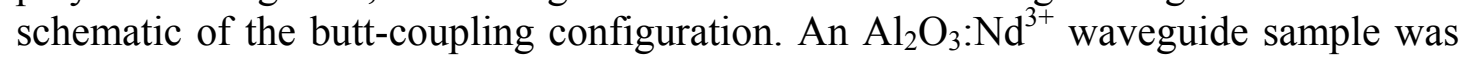
inserted between two polymer sections. Both the pump and signal light were coupled into a channel of the first polymer waveguide sample (Polymer I), propagated through 
Polymer I, then coupled into and out of a channel of the $\mathrm{Al}_{2} \mathrm{O}_{3}: \mathrm{Nd}^{3+}$ waveguide sample, and finally coupled out from the channel of the second polymer waveguide sample (Polymer II). Using the pump probe method, the internal net gain achieved in these three waveguides can be experimentally measured.

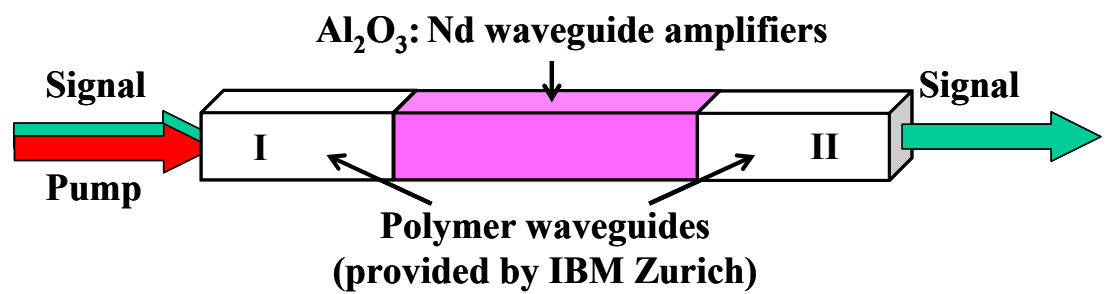

Fig. 6.6. Schematic of the butt-coupling configuration of $\mathrm{Al}_{2} \mathrm{O}_{3}: \mathrm{Nd}^{3+}$ and polymer waveguides in FR4.

In order to achieve internal net gain in such a system, in addition to the propagation losses in the three waveguides, the coupling losses at the two interfaces between polymer and $\mathrm{Al}_{2} \mathrm{O}_{3}: \mathrm{Nd}^{3+}$ waveguides need to be compensated by the gain accumulated in the $\mathrm{Al}_{2} \mathrm{O}_{3}: \mathrm{Nd}^{3+}$ amplifier. $\mathrm{A}$ three-dimensional (3D) Beam Propagation Method (BPM) [136] was applied to study and optimize the coupling between the two types of waveguides at $880 \mathrm{~nm}$. All the modes propagating through the channel waveguides were taken into account during the simulation. The coupling loss at the first interface (Polymer I to $\mathrm{Al}_{2} \mathrm{O}_{3}: \mathrm{Nd}^{3+}$ ) and second interface (Polymer II to $\mathrm{Al}_{2} \mathrm{O}_{3}: \mathrm{Nd}^{3+}$ ) have been calculated separately. The total coupling loss was obtained by adding the two parts together. The polymer waveguides of channel thickness $6 \mu \mathrm{m}$ were chosen, while the thickness of $\mathrm{Al}_{2} \mathrm{O}_{3}: \mathrm{Nd}^{3+}$ waveguides was varied from 1 to 5 $\mu \mathrm{m}$. The width of the polymer and $\mathrm{Al}_{2} \mathrm{O}_{3}: \mathrm{Nd}^{3+}$ channel waveguides were assumed to be the same during calculations. Figures 5.7(a) and (b) show the calculated coupling loss at the two waveguide interfaces as well as the total coupling loss versus layer thickness of the $\mathrm{Al}_{2} \mathrm{O}_{3}: \mathrm{Nd}^{3+}$ waveguides for channel widths of $6 \mu \mathrm{m}$ and $8 \mu \mathrm{m}$, respectively.

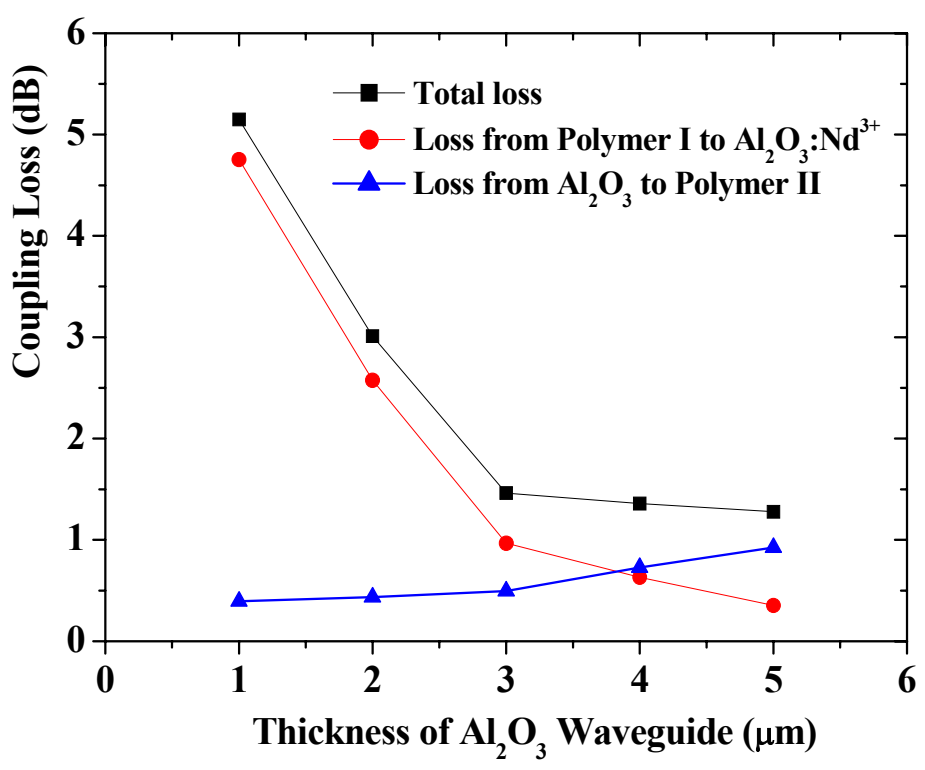

(a) 


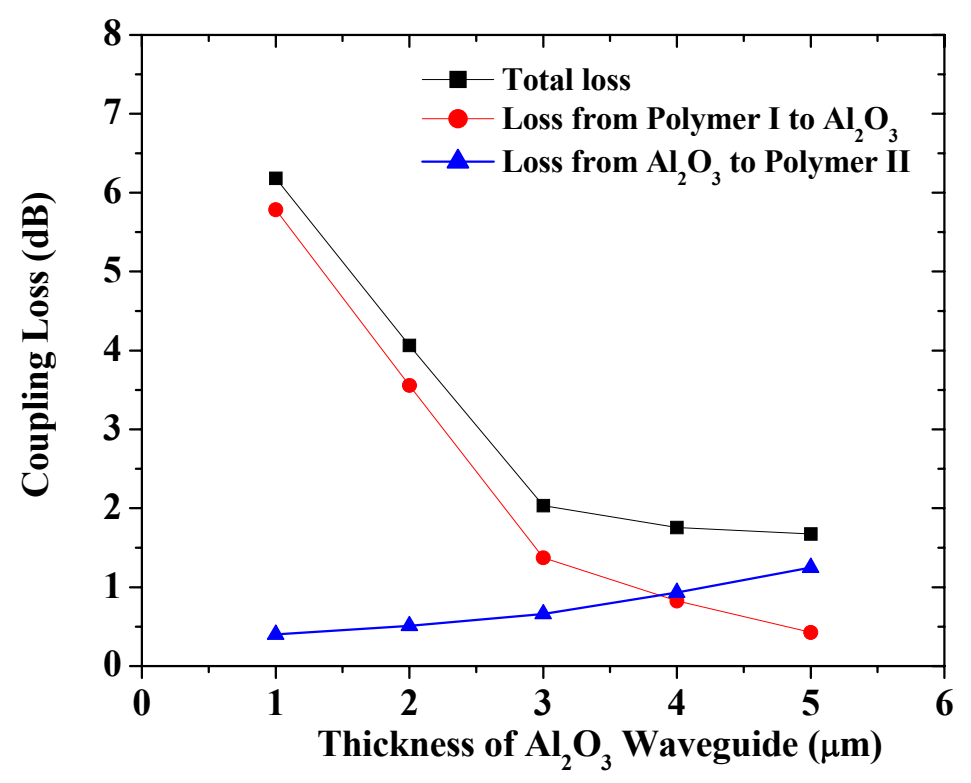

(b)

Fig. 6.7. Calculated coupling loss at $880 \mathrm{~nm}$ between polymer and $\mathrm{Al}_{2} \mathrm{O}_{3}: \mathrm{Nd}^{3+}$ waveguides with channel widths of (a) $6 \mu \mathrm{m}$ and (b) $8 \mu \mathrm{m}$.

The coupling loss between Polymer I and $\mathrm{Al}_{2} \mathrm{O}_{3}: \mathrm{Nd}^{3+}$ waveguides is mainly due to the mismatch of the thickness of the two waveguides and the corresponding losses experienced by high-order modes when propagating from Polymer I to $\mathrm{Al}_{2} \mathrm{O}_{3}: \mathrm{Nd}^{3+}$ waveguide. The loss is significantly reduced by increasing the thickness of the $\mathrm{Al}_{2} \mathrm{O}_{3}: \mathrm{Nd}^{3+}$ waveguide. The coupling loss between Polymer I and a 3- $\mu \mathrm{m}$-thick $\mathrm{Al}_{2} \mathrm{O}_{3}: \mathrm{Nd}^{3+}$ waveguide varied between 0.97 and $1.37 \mathrm{~dB}$.

The coupling loss at the interface between $\mathrm{Al}_{2} \mathrm{O}_{3}: \mathrm{Nd}^{3+}$ and Polymer II waveguides was much lower and increased slightly with increasing the layer thickness of the $\mathrm{Al}_{2} \mathrm{O}_{3}: \mathrm{Nd}^{3+}$ waveguide.

According to the calculation of coupling loss at the two interfaces, when the thickness of polymer channel waveguides is $6 \mu \mathrm{m}$, a total coupling loss below $2 \mathrm{~dB}$ is expected with $\mathrm{Al}_{2} \mathrm{O}_{3}: \mathrm{Nd}^{3+}$ channels thicker than $3 \mu \mathrm{m}$. A total coupling loss of 1.5-2.0 $\mathrm{dB}$ was expected at the two interfaces of a $3-\mu \mathrm{m}$-thick $\mathrm{Al}_{2} \mathrm{O}_{3}: \mathrm{Nd}^{3+}$ waveguide. When the thickness of the $\mathrm{Al}_{2} \mathrm{O}_{3}: \mathrm{Nd}^{3+}$ channel is further increased from $3 \mu \mathrm{m}$ to $5 \mu \mathrm{m}$, the total coupling loss is only slightly decreased.

The coupling loss between the two waveguides can be experimentally estimated by measuring the output intensity of the first and second waveguide, $I_{1}$ and $I_{2}$. Knowing the total background propagation and absorption loss $\alpha_{w 2}$ in $\mathrm{dB} / \mathrm{cm}$, and the propagation length $l_{\mathrm{w} 2}$ of the second waveguide, the coupling loss $L_{c}$ in $\mathrm{dB}$ can be determined as follows:

$$
L_{c}=10 \cdot \log \left(\frac{I_{1}}{I_{2}}\right)-\alpha_{w 2} \cdot l_{w 2}
$$


The coupling loss between a $6 \times 6-\mu \mathrm{m}^{2}$ polymer waveguide and a $3 \times 6-\mu \mathrm{m}^{2}$ polymer waveguide measured by this approach was $1.33 \mathrm{~dB}$, which agrees well with the value estimated from the simulation.

\subsubsection{Waveguide Tapers in $\mathrm{Al}_{2} \mathrm{O}_{3}: \mathrm{Nd}^{3+}$}

The minimum width of the polymer waveguides that can be achieved is limited by fabrication and the increase of the waveguide propagation loss with decreasing width. Besides, the waveguide geometries are even larger in currently investigated optical backplanes (typically $50 \times 50 \mu \mathrm{m}^{2}$ ). On the other hand, small waveguide geometries are required to achieve sufficient optical amplification. One possible solution is tapering down the waveguide geometry in the active part to improve the pump intensity. A linear horizontal taper waveguide was designed for $\mathrm{Al}_{2} \mathrm{O}_{3}: \mathrm{Nd}^{3+}$ waveguides and is depicted in Fig. 6.8. The width of the channel waveguide was first tapered down for achieving high gain and then tapered back to reduce the outcoupling losses. The two tapers at the two ends of the channel waveguide were designed to be symmetric.

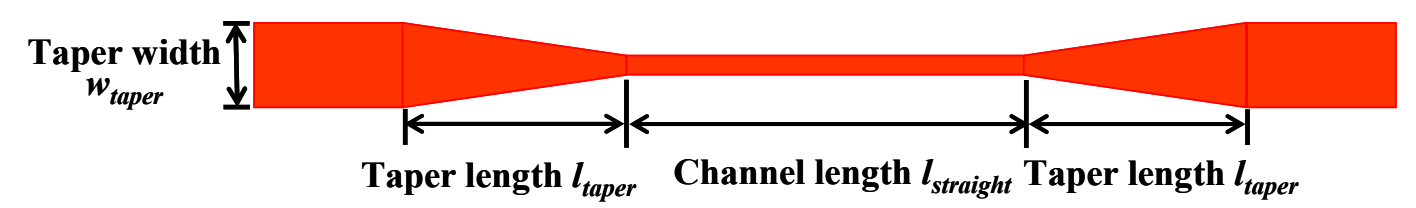

Fig. 6.8. Top view of the design of an $\mathrm{Al}_{2} \mathrm{O}_{3}: \mathrm{Nd}^{3+}$ tapered waveguide.

The length of the straight channel waveguide amplifier part $l_{\text {straight }}$ was varied for the optimization of optical gain with different $\mathrm{Nd}^{3+}$ concentrations and pump powers. To reduce re-absorption losses in the taper part, the length of the taper part $l_{\text {taper }}$ should be designed as short as possible. On the other hand, a certain length of the taper part is required to keep a low taper loss. The taper length was optimized by minimizing the taper losses $\alpha_{\text {taper }}$ in the waveguide as calculated by a 2D BPM at $880 \mathrm{~nm}$. Figures 6.9 (a) and (b) show the optical loss of tapers with a width of $8 \mu \mathrm{m}$ and $10 \mu \mathrm{m}$, respectively, as a function of taper length. The width of the channel waveguides was tapered down to $1.5,2.0$, and $2.5 \mu \mathrm{m}$.

According to the 2D BPM calculations, the optical loss reduced to a low level and remained almost constant for taper lengths longer than $500 \mu \mathrm{m}$. Therefore, taper lengths of $500 \mu \mathrm{m}$ and $700 \mu \mathrm{m}$ were chosen for the design of the mask. 


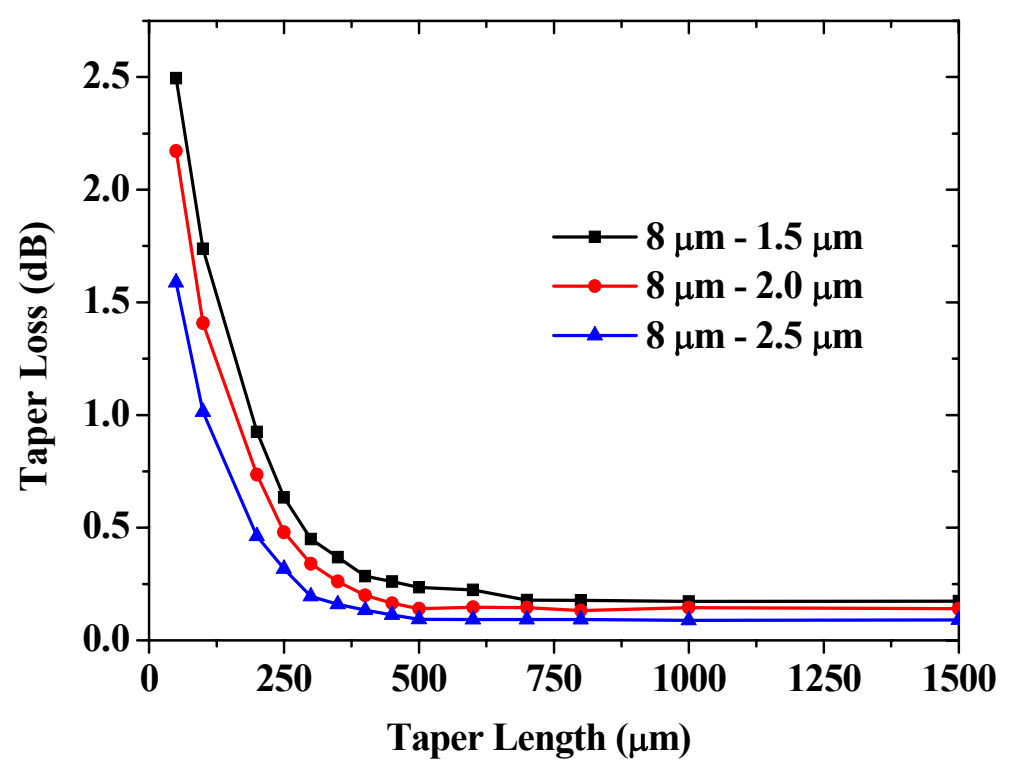

(a)

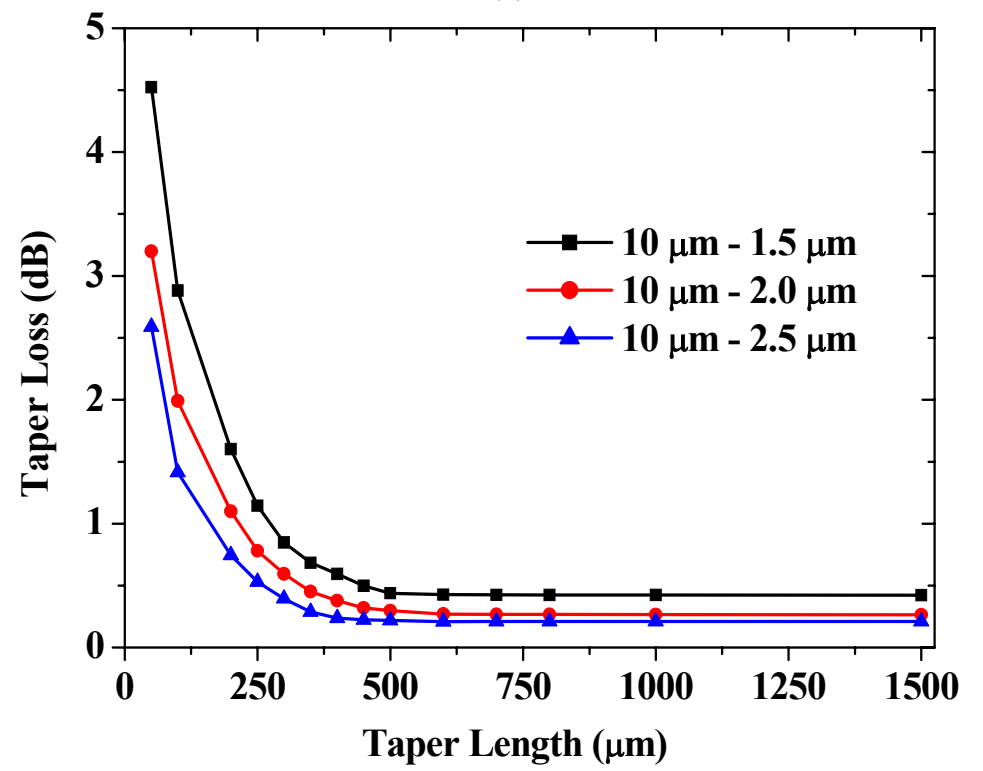

(b)

Fig. 6.9. Taper loss versus taper length at $880 \mathrm{~nm}$ in $\mathrm{Al}_{2} \mathrm{O}_{3}: \mathrm{Nd}^{3+}$ channel taper waveguides with a taper width of (a) $8 \mu \mathrm{m}$ and (b) $10 \mu \mathrm{m}$.

To experimentally investigate the extra optical loss introduced by the tapers, a similar-width straight channel waveguide was used as a reference. Its channel width was the same as the width of the taper and the same propagation length of the total taper channel length was used. The waveguide propagation losses in the waveguide with and without tapers are assumed to be the same. Since the width of the two channel waveguides at the starting part is the same, the same coupling efficiency can be achieved from polymer to these two waveguide. Therefore, the loss from the tapers can be determined by comparing the output intensity of the two waveguides.

\subsubsection{Side Pumping Coupler}

The low damage threshold of the polymer materials limits the pump power that can be coupled into the polymer waveguides. In addition, the pump power is attenuated due 
to the propagation loss in the polymer waveguide and coupling loss between the two waveguides. To improve the efficiency of the pump, a side pumping coupler was designed to couple the pump light directly into the $\mathrm{Al}_{2} \mathrm{O}_{3}: \mathrm{Nd}^{3+}$ waveguide. Figure 6.10 shows the design of a side pump coupler.

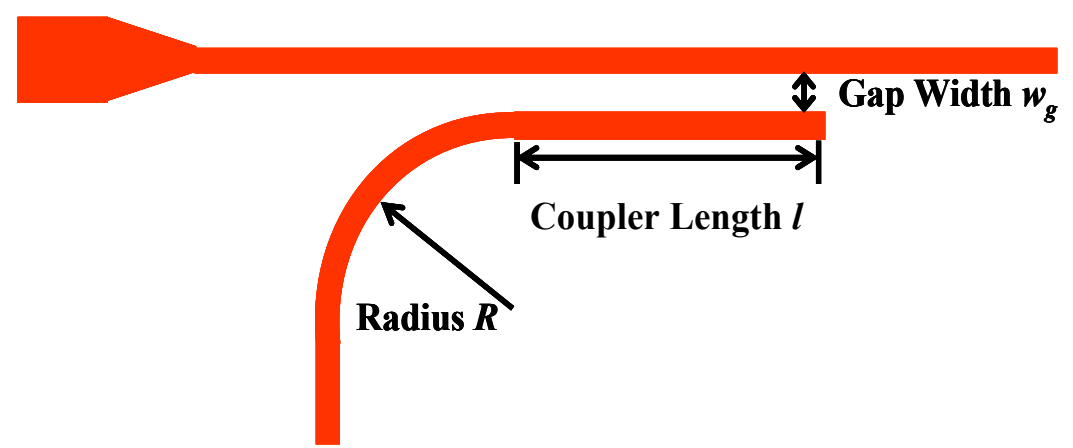

Fig. 6.10. The design of a side pump coupler.

In order to couple light from one waveguide to another typically a directional coupler is used. Because the two waveguides are closely spaced, the evanescent tail of the optical mode propagating in one waveguide interacts with the other waveguide. For a coupling region geometry shown in Fig. 6.10, the coupling coefficient $\kappa$ can be calculated using

$$
\kappa=\sin \left(\frac{\pi}{2} \frac{l+l_{\text {end }}}{l_{\text {coupling }}}\right),
$$

where $l$ is the length of the coupler.

$$
l_{\text {coupling }}=\frac{2 \pi}{\kappa}=\frac{\lambda_{0}}{2 \cdot\left|N_{\text {eff } 1}-N_{\text {eff } 2}\right|}
$$

is the coupling length required for completely coupling light of wavelength $\lambda_{0}$ from one waveguide to another [137]. The effective refractive index of the coupled modes $N_{\text {eff }}$ can be found using a mode solver. The end length $l_{\text {end }}$ is the contribution in the total coupling length of the bend sections and is given by [138]

$$
l_{\text {end }}=\sqrt{2 \pi R w_{g}},
$$

where $R$ is the bend radius and $w_{g}$ is the gap width between the port waveguide and the straight section.

Bend radii of $300 \mu \mathrm{m}$ and $450 \mu \mathrm{m}$ were chosen to guarantee a low bending loss for channel widths of $1 \mu \mathrm{m}$ and $2 \mu \mathrm{m}$, respectively. Figure 6.11 shows the 
coupling coefficient versus the length of $\left(l+l_{\text {end }}\right)$. To achieve a high coupling coefficient $(\sim 0.62)$ at the pump wavelength, $800 \mathrm{~nm}$, as well as a low coupling coefficient $(\sim 0.21)$ at the signal wavelength, $880 \mathrm{~nm}$, coupler lengths of $1369 \mu \mathrm{m}$ and $1962 \mu \mathrm{m}$ were designed for channel widths of $1 \mu \mathrm{m}$ and $2 \mu \mathrm{m}$, receptively, with a gap width of $2 \mu \mathrm{m}$.

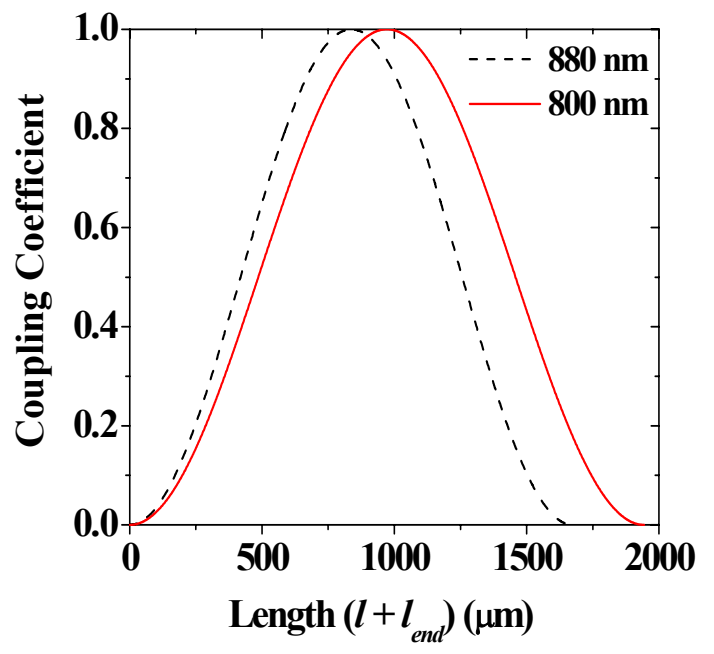

(a)

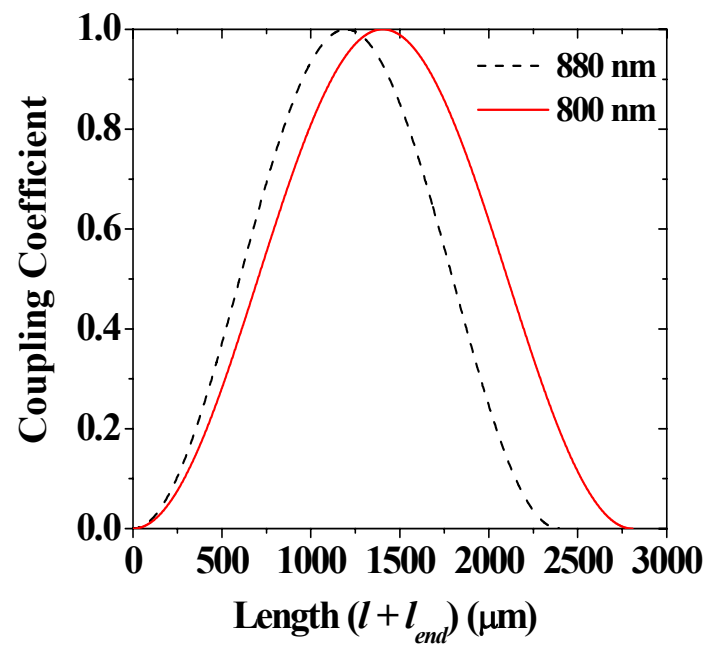

(b)

Fig. 6.11. Coupling coefficient of side pumping couplers versus the length of $\left(l+l_{\text {end }}\right)$ : (a) bend radius $300 \mu \mathrm{m}$ and channel width $1 \mu \mathrm{m}$; (b) bend radius $450 \mu \mathrm{m}$ and channel width $2 \mu \mathrm{m}$.

\subsection{Demonstration of Waveguide Amplifiers Integrated in Optical Backplanes}

\subsubsection{Gain Measurement}

The first demonstration of waveguide amplification was carried out with one polymer sample and one $\mathrm{Al}_{2} \mathrm{O}_{3}: \mathrm{Nd}^{3+}$ sample as depicted in Fig. 6.12. Two movable stages were used to optimize coupling of the samples. The end facets of the two waveguides were placed as close as possible, and a fluorinated index-matching fluid with refractive index of 1.57 was applied in between.

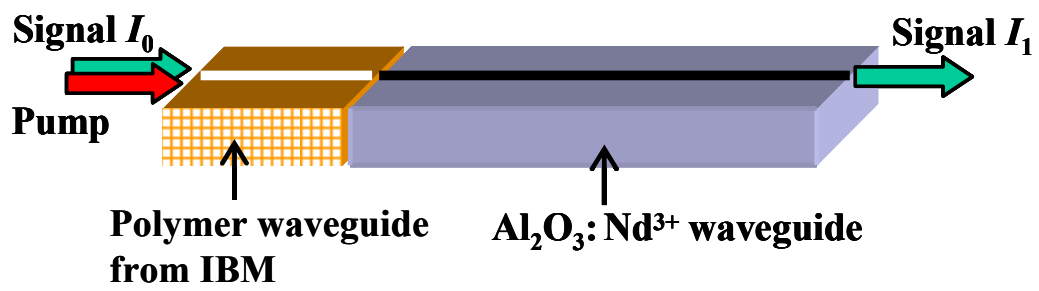

Fig. 6.12. Schematic of the demonstration of optical amplification in optical backplanes

The small signal gain was measured using the pump-probe method as described in Section 4.2. A Ti:Sapphire laser operating at $800 \mathrm{~nm}$ was used as the pump source and a diode laser at $880 \mathrm{~nm}$ was applied as the signal source. Pump light and signal light modulated by a mechanical chopper were combined and coupled into 
and out of the waveguides using two $10 \times$ microscope objectives. The unabsorbed pump light coupled out of the waveguide was blocked by a high-pass filter (RG850), while the transmitted signal light was measured by a germanium photodiode and amplified with the lock-in technique. The signal enhancement was determined by measuring the ratio of the transmitted signal intensities $I_{p}$ and $I_{u}$ in the pumped and unpumped case, respectively. By subtracting the total optical loss $L$ in $\mathrm{dB}$, the internal net gain was obtained by calculating the small-signal-gain coefficient in $\mathrm{dB}$ using the equation

$$
G_{\text {int ernal }}=10 \cdot \log _{10}\left(I_{p} / I_{u}\right)-L
$$

where $10 \cdot \log _{10}\left(I_{p} / I_{u}\right)$ is the signal enhancement. The total optical loss $L$ is the sum of all the relevant optical losses:

$$
L=\alpha_{\text {polymer }} \cdot l_{\text {polymer }}+\alpha_{A l 2 O 3} \cdot l_{A l 2 O 3}+L_{\text {coupling }}+L_{\text {taper }},
$$

where $\alpha_{\text {polymer }}$ is the background propagation loss coefficient of the polymer waveguide, $\alpha_{A l 2 O 3}$ is the background propagation and absorption loss coefficient of the $\mathrm{Al}_{2} \mathrm{O}_{3}: \mathrm{Nd}^{3+}$ waveguide, $L_{\text {coupling }}$ is the coupling loss between the two waveguides, $L_{\text {taper }}$ is the loss in tapers, and $l_{\text {polymer }}$ and $l_{A l 2 O 3}$ are the channel length of the polymer and $\mathrm{Al}_{2} \mathrm{O}_{3}: \mathrm{Nd}^{3+}$ waveguide, respectively.

\subsubsection{Gain Results}

A $6 \times 6-\mu \mathrm{m}^{2}$ polymer channel waveguide was used in the demonstration device, and the light was coupled into different $\mathrm{Al}_{2} \mathrm{O}_{3}: \mathrm{Nd}^{3+}$ waveguides with and without tapers after propagating through the polymer waveguide. Two 3- $\mu$ m-thick $\mathrm{Al}_{2} \mathrm{O}_{3}: \mathrm{Nd}^{3+}$ waveguide samples with $\mathrm{Nd}^{3+}$ concentrations of 0.38 and $0.91 \times 10^{20} \mathrm{~cm}^{3}$ were used for the measurements. The coupling losses from a $6 \times 6-\mu \mathrm{m}^{2}$ polymer waveguide to $3 \times 6-\mu \mathrm{m}^{2}$ and $3 \times 8-\mu \mathrm{m}^{2} \mathrm{Al}_{2} \mathrm{O}_{3}: \mathrm{Nd}^{3+}$ waveguides were measured to be 1.33 and $1.26 \mathrm{~dB}$ at $880 \mathrm{~nm}$, respectively. The measured taper loss at $880 \mathrm{~nm}$ from an 8 - $\mu \mathrm{m}$-wide channel tapered down to channels with width of $1.5-2.5 \mu \mathrm{m}$ is almost the same, approximately $0.32 \mathrm{~dB}$.

By directly coupling the pump and signal light from the polymer waveguide into $\mathrm{Al}_{2} \mathrm{O}_{3}: \mathrm{Nd}^{3+}$ waveguides with a width of $6 \mu \mathrm{m}$ without tapers, an internal gain of $0.12 \mathrm{~dB}$ was demonstrated in a 2-cm-long sample by pumping through the polymer waveguide with a launched pump power of $80 \mathrm{~mW}$. Table 6.3 shows the measured gain with different $\mathrm{Nd}^{3+}$ concentrations and channel lengths.

Only very low internal net gain has been achieved using $\mathrm{Al}_{2} \mathrm{O}_{3}: \mathrm{Nd}^{3+}$ waveguides without tapers due to the large active waveguide geometry and low pumping intensity. To improve the pump intensity in the active region, the light from the polymer waveguide was coupled into $\mathrm{Al}_{2} \mathrm{O}_{3}: \mathrm{Nd}^{3+}$ waveguides with a channel waveguide width of $8 \mu \mathrm{m}$. Its width was then tapered down to $1.5-2.5 \mu \mathrm{m}$. Table 6.4 shows the internal net gain measured in different tapered channel widths and lengths 
by coupling light via a $8-\mu \mathrm{m}$-wide taper. A maximum internal net gain of $0.42 \mathrm{~dB}$ was observed.

Table 6.3. Internal net gain at $880 \mathrm{~nm}$ at a launched pump power of $80 \mathrm{~mW}$ in 3- $\mu \mathrm{m}$-thick and 6- $\mu \mathrm{m}-$ wide $\mathrm{Al}_{2} \mathrm{O}_{2}: \mathrm{Nd}^{3+}$ waveguides.

\begin{tabular}{ccccc}
\hline \hline $\begin{array}{c}\mathbf{N d}^{3+} \text { conc. } \\
\left(\mathbf{1 0}^{\mathbf{2 0}} \mathbf{c m}^{\mathbf{3}}\right)\end{array}$ & $\begin{array}{c}\text { Channel length } \\
\text { of } \mathbf{A l}_{\mathbf{2}} \mathbf{O}_{\mathbf{3}}: \mathbf{N d} \mathbf{3}^{\mathbf{3}} \\
\mathbf{( c m )}\end{array}$ & $\begin{array}{c}\text { Measured total loss } \\
\mathbf{( d B})\end{array}$ & $\begin{array}{c}\text { Signal enhancement } \\
(\mathbf{d B})\end{array}$ & $\begin{array}{c}\text { Internal net gain } \\
(\mathbf{d B})\end{array}$ \\
\hline \multirow{2}{*}{0.38} & 2 & 3.43 & 2.80 & -0.83 \\
& 4 & 5.53 & 4.84 & -0.69 \\
\hline \multirow{2}{*}{0.91} & 2 & 4.55 & 4.67 & 0.12 \\
& 4 & 7.77 & 5.96 & -1.81 \\
\hline
\end{tabular}

Table 6.4. Internal net gain at $880 \mathrm{~nm}$ for a launched pump power of $80 \mathrm{~mW}$ in $6 \times 6-\mu \mathrm{m}^{2}$ polymer waveguides and 3- $\mu$ m-thick $\mathrm{Al}_{2} \mathrm{O}_{2}: \mathrm{Nd}^{3+}$ waveguides laterally tapered from $8 \mu \mathrm{m}$ down to channel widths given below. $\mathrm{Nd}^{3+}$ concentration: (a) $0.38 \times 10^{20} \mathrm{~cm}^{-3}$; (b) $0.91 \times 10^{20} \mathrm{~cm}^{-3}$.

(a)

\begin{tabular}{ccccc}
\hline \hline $\begin{array}{c}\mathbf{A l}_{2} \mathbf{O}_{3}: \mathbf{N d}^{3+} \\
\text { Length }(\mathbf{c m})\end{array}$ & $\begin{array}{c}\text { Channel } \\
\text { Width }(\boldsymbol{\mu m})\end{array}$ & $\begin{array}{c}\text { Measured total loss } \\
(\mathbf{d B})\end{array}$ & $\begin{array}{c}\text { Signal enhancement } \\
(\mathbf{d B})\end{array}$ & $\begin{array}{c}\text { Internal net gain } \\
(\mathbf{d B})\end{array}$ \\
\hline \multirow{2}{*}{4} & 1.5 & & 3.05 & -2.05 \\
& 2.0 & 5.10 & 3.80 & -1.30 \\
& 2.5 & & 4.72 & -0.37 \\
\hline \multirow{2}{*}{5} & 1.5 & \multirow{3}{*}{5.98} & 4.10 & -1.88 \\
& 2.0 & & 5.59 & -0.39 \\
& 2.5 & & 6.25 & 0.27 \\
\hline \multirow{2}{*}{6} & 1.5 & \multirow{2}{*}{6.76} & 3.84 & -2.92 \\
& 2.0 & & 5.29 & -1.47 \\
& 2.5 & & 6.03 & -0.73 \\
\hline
\end{tabular}

(b)

\begin{tabular}{ccccc}
\hline \hline $\begin{array}{c}\mathrm{Al}_{2} \mathbf{O}_{3}: \mathbf{N d}^{3+} \text { Channel } \\
\text { Length }(\mathbf{c m})\end{array}$ & $\begin{array}{c}\text { Measured total loss } \\
\text { Width }(\boldsymbol{\mu m})\end{array}$ & $\begin{array}{c}\text { Signal enhancement } \\
(\mathbf{d B})\end{array}$ & $\begin{array}{c}\text { Internal net gain } \\
(\mathbf{d B})\end{array}$ \\
\hline \multirow{2}{*}{2} & 1.5 & \multirow{3}{*}{4.48} & 3.02 & -1.46 \\
& 2.0 & & 3.81 & -0.67 \\
& 2.5 & & 4.30 & -0.18 \\
\hline \multirow{2}{*}{3} & 1.5 & \multirow{3}{*}{5.93} & 5.62 & -0.31 \\
& 2.0 & & 5.95 & 0.02 \\
& 2.5 & \multirow{2}{*}{7.38} & 6.35 & 0.42 \\
\hline \multirow{2}{*}{4} & 1.5 & & 6.34 & -1.02 \\
& 2.0 & & 6.52 & -0.86 \\
& 2.5 & & 7.60 & 0.22 \\
\hline
\end{tabular}

The internal net gain can be further increased by increasing the pump power. The pump efficiency can be improved by pumping from the side of the $\mathrm{Al}_{2} \mathrm{O}_{3}: \mathrm{Nd}^{3+}$ 
waveguides described in Section 6.4.3. Since the coupling loss from the $\mathrm{Al}_{2} \mathrm{O}_{3}: \mathrm{Nd}^{3+}$ waveguide to the second polymer waveguide is much less, it is possible to have internal net gain in the whole system.

The results indicate that it is possible to achieve optical amplification by integration of active waveguides into passive polymer waveguides in optical backplanes. Although the measured internal net gain was still low, it can be improved in the future work. Thus a route toward integration of optical amplification in optical backplanes has been opened.

\subsection{Summary}

The feasibility of $\mathrm{Al}_{2} \mathrm{O}_{3}: \mathrm{Nd}^{3+}$ channel waveguides for use as waveguide amplifiers between polymer waveguides in optical backplanes has been investigated.

Large-core $\mathrm{Al}_{2} \mathrm{O}_{3}: \mathrm{Nd}^{3+}$ channel waveguides have been fabricated and optical amplification has been characterized. By optimization of the propagation length, a maximum gain of $2.03 \mathrm{~dB}$ has been observed in a 3- $\mu \mathrm{m}$-thick, 3-cm-long, and 2.5$\mu \mathrm{m}$-wide channel waveguide with $\mathrm{Nd}^{3+}$ concentration of $0.91 \times 10^{20} \mathrm{~cm}^{-3}$ at a launched pump power of $55 \mathrm{~mW}$.

Polymer waveguides fabricated as optical backplanes by IBM Zurich were used for the demonstration of amplification in optical backplanes. $\mathrm{Al}_{2} \mathrm{O}_{3}: \mathrm{Nd}^{3+}$ channel waveguide samples were applied in between two polymer waveguide samples. Coupling losses between the two waveguides have been investigated to optimize the channel geometries of the two types of waveguides. A tapered waveguide has been designed to improve the pump intensities in the active waveguide region.

A maximum $0.42-\mathrm{dB}$ internal net gain has been demonstrated in a polymer waveguide coupled to an $\mathrm{Al}_{2} \mathrm{O}_{3}: \mathrm{Nd}^{3+}$ waveguide. The gain can be further improved by increasing the pump power. Therefore, a solution for compensating optical losses in optical interconnects has been provided. 
Chapter 6 


\section{Chapter 7}

\section{Conclusions}

Research performed within the frame of this thesis indicates that $\mathrm{Nd}^{3+}$ complex-doped 6-FDA/UVR and $\mathrm{Al}_{2} \mathrm{O}_{3}: \mathrm{Nd}^{3+}$ are well-suited as amplifiers and lasers for integrated active optical devices. In this chapter the main results of this thesis are summarized, conclusions are drawn, and an outlook for future work is provided. 
In this thesis neodymium-doped polymer and amorphous aluminum oxide waveguide amplifiers and lasers for integrated optical applications have been realized.

$\mathrm{Nd}^{3+}$-complex-doped, photo-defined polymer channel waveguides were realized on thermally oxidized silicon wafers with a simple and reproducible fabrication procedure by dividing the functionalities of photo-definition and active doping over two different polymers. Luminescence quenching by the high-energy vibrations present in polymers has been effectively reduced by choosing a fluorinated complex, $\mathrm{Nd}(\mathrm{TTA})_{3}$ phen, and dope it into a fluorinated polymer host, 6-FDA/UVR. The waveguides had very low loss in the visible and near-infrared wavelength range below $1.1 \mu \mathrm{m}$. A high internal net gain of $5.7 \mathrm{~dB} / \mathrm{cm}$ has been demonstrated at 1064 $\mathrm{nm}$, which is comparable with the best gain value that has been reported in Nd-dopedpolymer amplifiers around $1060 \mathrm{~nm}$. However, our polymer amplifiers have a much simpler fabrication procedure. In the wavelength range between 865 and $930 \mathrm{~nm}$, internal net optical gain with up to $2.8 \mathrm{~dB}$ gain was obtained at $873 \mathrm{~nm}$ by optimization of the $\mathrm{Nd}^{3+}$ concentration and channel waveguide length. The calculated and measured gain spectra of the ground-state transition show a good agreement. Gain per unit length of $2.0 \mathrm{~dB} / \mathrm{cm}$ has been measured at $873 \mathrm{~nm}$. To the best of our knowledge, this is the first report of Nd-doped polymer amplifiers at the ground-state transition. With these results a route toward low-cost integrated devices with optical gain has been opened.

Thermal stability is the main problem of polymer active devices, which limits the optical power in the waveguides. To solve this problem, the thermally more stable amorphous aluminum oxide was investigated as a host material. $\mathrm{Al}_{2} \mathrm{O}_{3}: \mathrm{Nd}^{3+}$ films were deposited on thermally oxidized silicon wafers using reactive co-sputtering, and RIE was applied to realize waveguide structures in the films. Channel waveguides fabricated by these techniques have high transparency and low background propagation losses from visible to infrared wavelengths. High-gain waveguide amplifiers have been demonstrated. At the investigated signal wavelengths of $880 \mathrm{~nm}$, $1064 \mathrm{~nm}$, and $1330 \mathrm{~nm}$ small-signal gain of $1.57 \mathrm{~dB} / \mathrm{cm}, 6.30 \mathrm{~dB} / \mathrm{cm}$, and $1.93 \mathrm{~dB} / \mathrm{cm}$, respectively, has been measured for individually optimized $\mathrm{Nd}^{3+}$ concentrations. Furthermore, a maximum gain of $3.0 \mathrm{~dB}, 14.4 \mathrm{~dB}$, and $5.1 \mathrm{~dB}$, respectively, has been obtained. On the ground-state transition, net optical gain has been demonstrated across the wavelength range of $865-930 \mathrm{~nm}$, with good agreement between calculated and measured gain spectra. Our $\mathrm{Al}_{2} \mathrm{O}_{3}: \mathrm{Nd}^{3+}$ waveguide amplifiers exhibit optical gain at $1064 \mathrm{~nm}$ on the ${ }^{4} \mathrm{~F}_{3 / 2} \rightarrow{ }^{4} \mathrm{I}_{11 / 2}$ transition, which is comparable to that of Nd-doped glass waveguides reported in the literature, but have an advantage in terms of highpackaging density due to the high refractive index contrast. Moreover, this work is the first demonstration of the complete gain spectrum on the ground-state transition around $865-930 \mathrm{~nm}$ as well as internal net gain at $1330 \mathrm{~nm}$ for telecommunication in Nd-doped glass-based waveguides. The wavelength range of optical gain in such amplifiers can be extended by doping with other rare-earth ions.

With these results for polymer and aluminium oxide waveguides, a route toward low-cost integrated devices with optical gain has been opened. Higher gain can be achieved by extending the waveguide length and increasing the pump power launched into these waveguides. With the gain simulated by a rate-equation model, the ETU parameter of $\mathrm{Nd}^{3+}$ in these two materials has been studied and the values have been determined. The obtained ETU parameters of $\mathrm{Nd}^{3+}$ in the polymer are 
slightly smaller than values of ETU parameters in many other $\mathrm{Nd}^{3+}$-doped host materials, which might be due to the isolation of each $\mathrm{Nd}^{3+}$ ion by its organic ligands increasing the minimum distance and reduces the interaction between neighboring $\mathrm{Nd}^{3+}$ ions. $\mathrm{Al}_{2} \mathrm{O}_{3}: \mathrm{Nd}^{3+}$, on the other hand, exhibits a higher ETU parameter than the majority of glass, polymer, and crystalline materials, which might be an indication of a close distance between two $\mathrm{Nd}^{3+}$ ions in $\mathrm{Al}_{2} \mathrm{O}_{3}$. Comparing the two types of waveguide amplifiers, $\mathrm{Nd}^{3+}$-complex-doped polymer waveguide amplifiers are easily fabricated and have large emission cross sections, which result in high gain at low pump intensities. However, the main disadvantage of polymer waveguide amplifiers is the low damage threshold compared with inorganic materials, thus limiting the pump power that can be applied to active devices. In addition, the propagation loss of polymer waveguides increases dramatically at the wavelength longer than $1.1 \mu \mathrm{m}$ due to the intrinsic absorption of the polymer. On the other hand, although the emission cross section of $\mathrm{Nd}^{3+}$ in $\mathrm{Al}_{2} \mathrm{O}_{3}$ is lower and the background losses of $\mathrm{Al}_{2} \mathrm{O}_{3}: \mathrm{Nd}^{3+}$ channels at visible and near infrared are a little bit higher, the good thermal stability and small waveguide size due to the high-refractive-index contrast make $\mathrm{Al}_{2} \mathrm{O}_{3}: \mathrm{Nd}^{3+}$ waveguides well-suited for integrated active devices. The waveguide devices based on the two materials have been shown to be well suited for providing optical gain for integrated optical applications, e.g. loss-less data transmission in optical interconnects and telecommunication, signal enhancement in integrated Raman spectroscopy, or lasers integrated into opto-fluidic chips.

Furthermore, laser experiments of polymer channel waveguides have resulted in lasers operating at the four-level as well as the quasi-three-level transition near $1060.2 \mathrm{~nm}$ and $878 \mathrm{~nm}$, respectively. These lasers exhibit remarkable lifetime stability, as they can withstand at least two hours of uninterrupted operation when pumping with up to $150 \mathrm{~mW}$. The maximum output power of the polymer laser was $\sim 1 \mathrm{~mW}$. This is the first demonstration of a rare-earth-ion-doped polymer waveguide laser as well as a $\mathrm{CW}$ solid polymer laser. The $\mathrm{CW}$ polymer waveguide laser developed in this work has a great application potential, e.g. for use as a miniaturized integrated light source for lab-on-chip applications involving infrared and quantumdot labeling in life sciences and medical diagnostics, or as a tool for fluorescence correlation spectroscopy.

One application of rare-earth-ion-doped waveguide amplifiers is optical amplification of embedded waveguides in optical backplanes. The feasibility of using $\mathrm{Al}_{2} \mathrm{O}_{3}: \mathrm{Nd}^{3+}$ channel waveguides as amplifiers between polymer waveguides in optical backplanes has been investigated. $\mathrm{Al}_{2} \mathrm{O}_{3}: \mathrm{Nd}^{3+}$ channel waveguides with a PECVD $\mathrm{SiO}_{2}$ layer as an upper cladding were placed in between two polymer waveguide samples to achieve optical amplification in optical waveguides on backplanes. The polysiloxane-based polymer channel waveguide samples fabricated on FR4 substrates for a demonstration experiment were provided by IBM Zurich. The coupling loss between the two waveguides has been investigated and the channel geometries of the two types of waveguides were optimized accordingly. A tapered waveguide has been designed to improve the pump intensities in the active waveguide region. A maximum of $0.42 \mathrm{~dB}$ internal net gain has been demonstrated in a polymer waveguide coupled to an $\mathrm{Al}_{2} \mathrm{O}_{3}: \mathrm{Nd}^{3+}$ waveguide. The gain can be further improved by increasing the pump power. Therefore, a solution for compensating optical loss in optical interconnects has been provided.

In conclusion, the $\mathrm{Nd}^{3+}$-doped waveguide materials developed in this work are attractive for integrated optical applications due to their high gain and ease of 
integration with integrated optical devices. Combination with other waveguide components to realize advanced functionalities, such as zero-loss splitters, ring lasers, and DFB lasers, will be promising for integrated optical applications. 


\section{References}

[1] S. Uhlig and M. Robertsson, "Limitations to and solutions for optical loss in optical backplanes", J. Lightwave Technol., vol. 24, pp. 1710-1712, 2006.

[2] J. Moisel, J. Guttmann, H. P. Huber, O. Krumpholz, and M. Rode, "Optical backplanes with integrated polymer waveguides", Opt. Eng., vol. 39, pp. 673-679, 2000.

[3] S. Lehmacher and A. Neyer, "Integration of polymer optical waveguides into printed circuit boards (PCB)", Electron. Lett., vol. 36, pp. 1052-1053, 2000.

[4] L. Dellmann, C. Berger, R. Beyeler, R. Dangel, M. Gmür, R. Hamelin, F. Horst, T. Lamprecht, N. Meier, T. Morf, S. Oggioni, M. Spreafico, R. Stevens, and B. J. Offrein, " $120 \mathrm{~Gb} / \mathrm{s}$ optical card-to-card interconnect link demonstrator with embedded waveguides", $57^{\text {th }}$ Electronic Components and Technology Conference Proceeding, May 29 - June 1, 2007, John Ascuaga's Nugget, Reno, Nevada, USA, pp. 1288-1293, 2007.

[5] C. H. Henry, G. E. Blonder, and R. F. Kazarinov, "Glass waveguides on silicon. for hybrid optical packaging", J. Lightwave Technol., vol. 7, pp. 1530-1539, 1989.

[6] M. Kawachi, "Silica waveguides on silicon and their application to integrated-optic components", Opt. Quantum Electron., vol. 22, pp 391-416, 1990.

[7] G. Grand, J. P. Jadot, H. Denis, S. Valette, A. Foumier, and A. M. Grouillet, "Low-loss PECVD silica channel waveguides for optical communications", Electron. Lett., vol. 26, pp 2135-2137, 1990.

[8] W. Chuang, C. Chang, C. Lai, and K. Lin, "Integrated-optics multimodeinterference wavelength fivision multiplexer for optical communication", Fiber Integrated Opt., vol. 18, pp. 93-104, 1999.

[9] A. N. Sloper, J. K. Deacon, and M. T. Falnagan, "A planar indium phosphate monomode waveguide evanescent field immunosensor", Sens. Actuators B, vol. 1, pp. 589-591, 1990.

[10] W. Lukosz, "Integrated optical chemical and direct biochemical sensors", Sens. Actuators B, vol. 29, pp. 37-50, 1995.

[11] R. Horváth, H. C. Pedersen, N. Skivesen, D. Selmeczi, and N. B. Larsen, "Optical waveguide sensor for on-line monitoring of bacteria", Opt. Lett., vol. 28, pp. 1233-1235, 2003.

[12] P. J. Caspers, G. W. Lucassen, E. A. Carter, H. A. Bruining, and G. J. Puppels, "In vivo confocal raman microspectroscopy of the skin: noninvasive determination of molecular concentration profiles", J. Invest. Dermatol., vol. 116, pp. 434-442, 2001.

[13] P. J. Caspers, G. W. Lucassen, and G. J. Puppels, "Combined in vivo confocal Raman spectroscopy and confocal microscopy of human skin", Biophys. J., vol. 85, pp. 572-580, 2003.

[14] F. Spedding and A. H. Daane, The Rare Earths, John Wiley \& Sons, New York, 1961.

[15] A. A. Kaminskii, Laser Crystals, Springer-Verlag, Berlin, 1979.

[16] M. J. F. Digonnet, Rare-earth-doped Fiber Laser and Amplifiers, second edition, revised and expanded, New York, Basel, Marcel Dekker Inc., 2001. 
[17] A. Yariv, Quantum Electronics, third edition, John Wiley \& Sons, New York, 1989.

[18] R. J. Mears, L. Reekie, I. M. Jauncey, and D. N. Payne: "Low-noise erbiumdoped fibre amplifier at $1.54 \mu \mathrm{m}$ ", Electron. Lett., vol. 23, pp.1026-1028, 1987.

[19] E. Desurvire, J. Simpson, and P. C. Becker, "High-gain erbium-doped traveling-wave fiber amplifier", Opt. Lett., vol. 12, pp. 888-890, 1987.

[20] L. Goldberg, J. P. Koplow, and D. A. V. Kliner, "Highly efficient 4-W Ybdoped fiber amplifier pumped by a broad-strip laser diode", Opt. Lett., vol. 24, pp. 673-675, 1999.

[21] J. Limpert, T. Schreiber, T. Clausnitzer, K. Zöllner, H.-J. Fuchs, E.-B. Kley, H. Zellmer, and A. Tünnermann, "High-power femtosecond Yb-doped fiber amplifier", Opt. Express, vol. 10, pp. 628-638, 2002.

[22] E. Snitzer, H. Po, F. Hakimi, R. Tumminelli, and B. C. McCollum, "Double clad, offset core Nd fiber laser", Optical Fiber Communication Conference, Paper PD5, 1988.

[23] X. Xu, "Properties of $\mathrm{Nd}^{3+}$-doped polymer optical fiber amplifiers", Opt. Commun., vol. 205, pp. 55-59, 2003.

[24] K. Kuriki and Y. Koike, "Plastic optical fiber lasers and amplifiers containing lanthanide complexes", Chem. Rev., vol.102, pp 2347-2356, 2002.

[25] H. Liang, Q. Zhang, Z. Zheng, H. Ming, Z. Li, J. Xu, B. Chen, and H. Zhao, "Optical amplification of Eu(DBM) $)_{3}$ Phen-doped polymer optical fiber", Opt. Lett., vol. 29, pp. 477-479, 2004.

[26] S. D. Jackson and T. A. King, "Operation of a 1.064- $\mu \mathrm{m}$ pumped Tm-Hodoped silica fiber laser”, IEEE J. Quantum Electron., vol. 34, pp. 1578-1587, 1998.

[27] D. S. Gill, A. A. Anderson, R. W. Eason, T. J. Warburton, and D. P. Shepherd, "Laser operation of an $\mathrm{Nd}: \mathrm{Gd}_{3} \mathrm{Ga}_{5} \mathrm{O}_{12}$ thin-film optical waveguide fabricated by pulsed laser deposition", Appl. Phys. Lett., vol. 69, pp. 10-12, 1996.

[28] E. Lallier, J. P. Pocholle, M. Papuchon, M. De Micheli, M. J. Li, Q. He, D. B. Ostrowsky, C. Grezes-Besset, and E. Pelletier, "Nd:MgO:LiNbO waveguide laser and amplifier", Opt. Lett., vol. 15, pp. 682-684, 1990.

[29] J. R. Lee, H. J. Baker, G. J. Friel, G. J. Hilton, and D. R. Hall, "Highaverage-power Nd:YAG planar waveguide laser that is face pumped by 10 laser diode bars", Opt. Lett., vol. 27, pp. 524-526, 2002.

[30] L. H. Slooff, A. van Blaaderen, A. Polman, G. A. Hebbink, S. I. Klink, F. C. J. M. Van Veggel, D. N. Reinhoudt, and J. W. Hofstraat, "Rare-earth doped polymers for planar optical amplifiers", J. Appl. Phys., vol. 91, pp. 3955-380, 2002.

[31] R. T. Chen, M. Lee, S. Natarajan, C. Lin, Z. Z. Ho, and D. Robinson, "Single-mode $\mathrm{Nd}^{3+}$-doped graded-index polymer waveguide amplifier", IEEE Photon. Technol. Lett., vol. 5, pp. 1328-1330, 1993.

[32] D. An, Z. Yue, and R. T. Chen, "Dual-functional polymeric waveguide with optical amplification and electro-optic modulation", Appl. Phys. Lett., vol. 72, pp. 2806-2807, 1998.

[33] G. Karve, B. Bihari, and R. T. Chen, "Demonstration of optical gain at 1.06 $\mu \mathrm{m}$ in a neodymium-doped polyimide waveguide", Appl. Phys. Lett., vol. 77, pp. 1253-1255, 2000. 
[34] C. Grivas, D. P. Shepherd, T. C. May-Smith, R. W. Eason, and M. Pollnau, "Single-transverse-mode Ti:sapphire rib waveguide laser", Opt. Express, vol. 13, pp. 210-215, 2005.

[35] Y. E. Romanyuk, C. N. Borca, M. Pollnau, S. Rivier, V. Petrov, and U. Griebner, "Yb-doped $\mathrm{KY}\left(\mathrm{WO}_{4}\right)_{2}$ planar waveguide laser", Opt. Lett., vol. 31, pp. 53-55, 2006.

[36] D. Geskus, S. Aravazhi, E. Bernhardi, C. Grivas, S. Harkema, K. Hametner, D. Günther, K. Wörhoff, and M. Pollnau, "Low-threshold, highly efficient $\mathrm{Gd}^{3+}, \mathrm{Lu}^{3+}$ co-doped $\mathrm{KY}\left(\mathrm{WO}_{4}\right)_{2}: \mathrm{Yb}^{3+}$ planar waveguide lasers", Laser Phys. Lett., vol. 6, pp. 800-805, 2009.

[37] R. Brinkmann, I. Baumann, M. Dinand, W. Sohler, and H. Suche, "Erbiumdoped single- and double-pass Ti: $\mathrm{LiNbO}_{3}$ waveguide amplifiers", IEEE $J$. Quantum Electron., vol. 30, pp. 2356-2360, 1994.

[38] W. H. Wong, E. Y. B. Pun, and K. S. Chan, " $\mathrm{Er}^{3+}-\mathrm{Yb}^{3+}$ codoped polymeric optical waveguide amplifiers", Appl. Phys. Lett., vol. 84, pp. 176-178, 2004.

[39] A. Q. Le Quang, R. Hierle, J. Zyss, I. Ledoux, I. Ledoux, G. Cusmai, R. Costa, A. Barberis, and S. M. Pietralunga, "Demonstration of net gain at $1550 \mathrm{~nm}$ in an erbium-doped polymer single mode rib waveguide", Appl. Phys. Lett., vol. 89, pp. 141124-141126, 2006.

[40] J. D. B. Bradley, F. Ay, K. Wörhoff, M. Pollnau, "Fabrication of low-loss channel waveguides in $\mathrm{Al}_{2} \mathrm{O}_{3}$ and $\mathrm{Y}_{2} \mathrm{O}_{3}$ layers by inductively coupled plasma reactive ion etching", Appl. Phys. B, vol. 89, pp. 311-318, 2007.

[41] K. Wörhoff, J. D. B. Bradley, F. Ay, D. Geskus, T. P. Blauwendraat, and M. Pollnau, "Reliable low-cost fabrication of low-loss $\mathrm{Al}_{2} \mathrm{O}_{3}: \mathrm{Er}^{3+}$ waveguides with 5.4-dB optical gain", IEEE J. Quantum Electron., vol. 45, pp. 454-461, 2009.

[42] J. D. B. Bradley, Ph.D. thesis, University of Twente, Enschede, 2009.

[43] D. Zhang, C. Chen, C. M. Chen, C. S. Ma, D. M. Zhang, S. Bo, and Z. Zhen, "Optical gain at $1535 \mathrm{~nm}$ in $\mathrm{LaF}_{3}: \mathrm{Er}, \mathrm{Yb}$ nanoparticle-doped organicinorganic hybrid material waveguide", Appl. Phys. Lett., vol. 91, pp 161109/1-3, 2007.

[44] G. Facchimi, A. Zappettini, A. Canali, M. Martinelli, G. Gabetta, and G. Tallarida, "Erbium-doped crystalline YAG planar and ridge waveguides on quartz and sapphire substrates: Deposition and material characterisation", Opt. Mater., vol. 17, pp. 251-254, 2001.

[45] T. Kitagawa, K. Hattori, K. Shuto, M. Yasu, M. Kobayashi, and M. Horiguchi, "Amplification in erbium-doped silica-based planar lightwave circuits", Electron. Lett., vol. 28, pp. 1818-1819, 1992.

[46] T. Ohtsuki, N. Peyghambarian, S. Honkanen, and S. I. Najafi, "Gain characteristics of a high-concentration $\mathrm{Er}^{3+}$-doped phosphate glass waveguide", J. Appl. Phys., vol. 78, pp. 3617-3621, 1995.

[47] U. Aoki, E. Ishikawa, and Y. Asahara, " $\mathrm{Nd}^{3+}$-doped glass waveguide amplifier at $1.054 \mu \mathrm{m}$ ", Electron. Lett., vol. 27, pp. 2351-2353, 1991.

[48] M. Ajroud, M. Haouari, H. Ben Ouada, H. Maaref, A. Brenier, and C. Garapon, "Investigation of the spectroscopic properties of $\mathrm{Nd}^{3+}$-doped phosphate glasses", J. Phys.: Condens. Matter, vol. 12, pp. 3181-3193, 2000.

[49] H. Ma, A. K.-Y. Jen, and L. R. Dalton, "Polymer-based optical waveguides: materials, processing, and devices", Adv. Mater.; vol. 14, pp. 1339-1365, 2002. 
[50] L. Eldada and L. W. Shacklette, "Advances in polymer integrated optics", IEEE J. Select. Topics Quantum Electron, vol. 6, pp. 54-68, 2000.

[51] W. H. Wong, Z. Zhou, and E. Y. B. Pun, "Thermally stable low loss polymeric optical waveguides using electron beam direct writing", Appl. Phys. Lett., vol. 78, pp. 2110-2112, 2001.

[52] M. B. J. Diemeer, L. T. H. Hilderink, R. Dekker, and A. Driessen, "Lowcost and low-loss multimode waveguide of photodefinable epoxy", IEEE Photon. Technol. Lett., vol. 18, pp. 1624-1626, 2006.

[53] J. Kobayashi, Y. Inoue, T. Matsuura, and T. Maruno, "Tunable and polarization-insensitive arrayed-waveguide grating multiplexer fabricated from fluorinated polyimides", IEEE Photon. Technol. Lett., vol. 12, pp. 407409, 2000.

[54] D. Chen, H. R. Fetterman, A. Chen, W. H. Steier, L. R. Dalton, W. Wand, and Y. Shi, "Demonstration of $110 \mathrm{GHz}$ electro-optic polymer modulators", Appl. Phys. Lett., vol. 70, pp. 3335-3337, 1997

[55] A. Costela, I. García-Moreno, and R. Sastre, "Polymeric solid-state dye lasers: Recent developments", Phys. Chem. Chem. Phys., vol. 5, pp. 47454763, 2003.

[56] I. D. W. Samuel and G. A. Turnbull, "Organic semiconductor lasers", Chem. Rev., vol. 107, pp. 1272-1295, 2007.

[57] R. Bornemann, U. Lemmer, and E. Thiel, "Continuous-wave solid-state dye laser", Opt. Lett., vol. 31, pp. 1669-1671, 2006.

[58] B. Whittaker, "Low threshold laser action of a rare earth chelate in liquid and solid host media", Nature, vol. 228, pp. 157-159, 1970.

[59] H. Taniguchi, J. Kido, M. Nishiya, ad S. Sasaki, "Europium chelate solid laser based on morphology-dependent resonances", Appl. Phys. Lett., vol. 67, pp. 1060-1062, 1995.

[60] M. K. Smit, G. A. Acket, and C. J. van der Laan, " $\mathrm{Al}_{2} \mathrm{O}_{3}$ films for integrated-optics", Thin Solid Films, vol. 138, pp. 171-181, 1986.

[61] G. N. van den Hoven, E. Snoeks, A. Polman, J. W. M. van Uffelen, Y. S. Oei, and M. K. Smit, "Photoluminescence characterization of Er-implanted $\mathrm{Al}_{2} \mathrm{O}_{3}$ films", Appl. Phys. Lett., vol. 62, pp. 3065-3067, 1993.

[62] G. N. van den Hoven, R. Koper, A. Polman, C. van Dam, J. W. M. van Uffelen, and M. K. Smit, "Net optical gain at $1.53 \mu \mathrm{m}$ in Er-doped $\mathrm{Al}_{2} \mathrm{O}_{3}$ waveguides on silicon", Appl. Phys. Lett., vol. 68, pp. 1886-1888, 1996.

[63] R. Serna and C. N. Afonso, "In situ growth of optically active erbium doped $\mathrm{Al}_{2} \mathrm{O}_{3}$ thin films by pulsed laser deposition", Appl. Phys. Lett., vol. 69, pp. 1541-1543, 1996.

[64] C. E. Chryssou and C. W. Pitt, " $\mathrm{Er}^{3+}$-doped $\mathrm{Al}_{2} \mathrm{O}_{3}$ thin films by plasmaenhanced chemical vapor deposition (PECVD) exhibiting a 55-nm optical bandwidth", IEEE J. Quantum Electron., vol. 34, pp. 282-285, 1998.

[65] S. Musa, H. J. van Weerden, T. H. Yau, and P. V. Lambeck, "Characteristics of Er-doped $\mathrm{Al}_{2} \mathrm{O}_{3}$ thin films deposited by reactive co-sputtering", IEEE J. Quantum Electron., vol. 36, pp. 1089-1097, 2000.

[66] K. Solehmainen, M. U. Kapulainen, P. Heimala, and K. Polamo, "Erbiumdoped waveguides fabricated with atomic layer deposition method", IEEE Photon. Technol. Lett., vol. 16, pp. 194-196, 2004.

[67] D. Miller, "Rationale and challenges for optical interconnects to electronic chips", Proc. IEEE, vol. 88, pp. 728-749, 2000. 
[68] E. Griese, "A high-performance hybrid electrical-optical interconnection technology for high-speed electronic systems", IEEE Trans. Adv. Packag., vol. 24, pp. 375-383, 2001.

[69] A. Naeemi, J. Xu, A. Mule, T. Gaylord, and J. Meindl, "Optical and electrical interconnect partition length based on chip-to-chip bandwidth maximization", IEEE Photon. Technol. Lett., vol. 16, pp. 1221-1223, 2004.

[70] C. Svensson, "Electrical interconnects revitalized", IEEE Trans. Very Large Scale Integr. (VLSI) Syst., vol. 10, pp. 777-788, 2002.

[71] R. R. Tummala, Fundamentals of Microsystems Packaging, McGraw-Hill, NewYork, 2001.

[72] C. Berger, M. A. Kossel, C. Menolfi, T. Morf, T. Toifl, and M. L. Schmatz, "High-density optical interconnects within large-scale systems", in Proc. SPIE_VCSELs and Optical Interconnects, vol. 4942, pp. 222-235, 2003.

[73] A. P. Thorne, Spectrophysics, 2nd ed, Chapman \& Hall, London, 1989.

[74] J. D. J. Ingle and S. R. Crouch, Spectrochemical Analysis, Prentice Hall, New Jersey, 1988.

[75] W. B. Fowler and D. L. Dexter, "Relation between absorption and emission probabilities in luminescent centers in ionic solids", Phys. Rev., vol. 128, pp. 2154-2165, 1962.

[76] D. E. McCumber, "Einstein relations connecting broadband emission and absorption spectra", Phys, Rev., vol. 136, pp. 954-957, 1964.

[77] W. J. Miniscalco and R. S. Quimby, "General procedure for the analysis of $\mathrm{Er}^{3+}$ cross sections", Opt. Lett., vol. 16, pp 258-260, 1991.

[78] S. A. Paye, L. L. Chase, L. K. Smith, W. L. Kway, and W. F. Krupke, "Infrared cross section measurements for crystals doped with $\mathrm{Er}^{3+}, \mathrm{Tm}^{3+}$, and $\mathrm{Ho}^{3+,}$, IEEE J. Quantum Electron., vol. 28, pp. 2619-2630, 1992.

[79] L. D. Deloach, S. A. Paye, L. L. Chase, L. K. Smith, W. L. Kway, and W. F. Krupke, "Evaluation of absorption and emission properties of $\mathrm{Yb}^{3+}$ doped crystals for laser applications", IEEE J. Quantum Electron., vol. 29, pp. 1179-1191, 1993.

[80] B. R. Judd, "Optical absorption intensities of rare-earth ions", Phys. Rev., vol. 127 , pp.750-761, 1962.

[81] G. S. Ofelt, "Intensities of crystal spectra of rare-earth ions", J. Chem. Phys., vol. 37, pp. 511-520, 1962.

[82] W. F. Krupke, "Induced-emission cross sections in neodymium laser glasses", IEEE J. Quantum Electronics, vol. QE-10, pp. 450-457, 1974.

[83] Y. Hasegawa, Y. Wada, S. Yanagida, "Strategies for the design of luminescent lanthanide (III) complexes and their photonic applications", $J$. Photochem. Rev., vol 5, pp. 183-202, 2004.

[84] J. R. Ryan and R. Beach, "Optical absorption and stimulated emission of neodymium in yttrium lithium fluoride", J. Opt. Soc. Am. B, vol. 9, pp. 1883-1887, 1992.

[85] R. S. Quimby and W. J. Miniscalco, "Modified Judd-Ofelt technique and application to optical transitions in $\mathrm{Pr}^{3+}$-doped glass", J. Appl. Phys., vol. 75, pp. 613-615, 1994.

[86] D. K. Sardar, J. B. Gruber, B. Zandi, J. A. Hutchinson, and C. W. Trussell, "Judd-Ofelt analysis of the $\mathrm{Er}^{3+}\left(4 \mathrm{f}^{11}\right)$ absorption intensities in phosphate glass: $\mathrm{Er}^{3+}, \mathrm{Yb}^{3+}$, , J. Appl. Phys., vol. 93, pp. 2041-2046, 2003.

[87] W. T. Carnall, P. R. Fields, and K. Rajnak, "Electronic energy levels in the trivalent lanthanide aquo ions", J. Chem. Phys., vol.49, pp. 4424-4442, 1968. 
[88] M. Pollnau, P. J. Hardman, W. A. Clarkson, and D. C. Hanna, "Upconversion, lifetime quenching, and ground-state bleaching in $\mathrm{Nd}^{3+}$ :LiYF 4 ", Opt. Commun., vol. 147, pp. 203-211, 1998.

[89] M. Pollnau, P. J. Hardman, M. A. Kern, W. A. Clarkson, and D. C. Hanna, "Upconversion-induced heat generation and thermal lensing in Nd:YLF and Nd:YAG", Phys. Rev. B, vol. 58, pp. 16076-16092, 1998.

[90] C. K. Jørgensen and R. Reisfeld, "Judd-Ofelt parameters and chemical bonding", J. Less-Common Metals, vol. 93, pp. 107-112, 1983.

[91] W. F. Krupke, "Radiative transition probabilities within the $4 \mathrm{f}^{3}$ ground configuration of Nd:YAG”, IEEE J. Quantum Electron., vol. 7, pp. 153-159, 1971.

[92] G. Wang, "Optical-transition probability of the $\mathrm{Nd}^{3+}$ ion in $\mathrm{GdAl}_{3}\left(\mathrm{BO}_{3}\right)_{4}$ crystal", J. Opt Soc Am B, vol. 18, pp. 173-175, 2001.

[93] B. Viana, M. Palazzi, and O. LeFol, "Optical characterization of $\mathrm{Nd}^{3+}$ doped sulphide glasses", J. Non-Crystalline Solids, vol. 215, pp. 96-102, 1997.

[94] H. Takebe, K. Yoshino, T. Murata, K. Morinaga, J. Hector, W. S. Brocklesby, D. W. Hewak, J. Wang, and D. N. Payne, "Spectroscopic properties of $\mathrm{Nd}^{3+}$ and $\mathrm{Pr}^{3+}$ in gallate glasses with low phonon energies", Appl. Opt., vol. 36, pp. 5839-5843, 1997.

[95] M. D. McGehee, R. Gupta, S. Veenstra, E. K. Miller, M. A. Diaz-Garcia, and A. J. Heeger, "Amplified spontaneous emission from photopumped films of a conjugated polymer", Phys. Rev. B, vol.58, pp. 7035-7039, 1998.

[96] V. I. Klimov and M. G. Bawendi, "Ultrafast carrier dynamics, optical amplification, and lasing in nanocrystal quantum dots", MRS Bulletin, vol. 12, pp. 998-1004, 2001.

[97] D. N. Payne, "Active fibres and optical amplifiers", Fiber Int. Opt., vol. 11, pp. 191-219, 1992.

[98] L. H. Slooff, A. Polman, S. I. Klink, L. Grave, F. C. J. M. van Veggel, and J. W. Hofstraat, "Concentration effects in the photodegradation of lissaminefunctionalized neodymium complexes in polymer waveguides", J. Opt. Soc. Am. B, vol. 18, pp. 1690-1694, 2001.

[99] F. Artizzu, L. Marchiò, M. L. Mercuri, L. Pilia, A. Serpe, F. Quochi, R. Orrù, F. Cordella, M. Saba, A. Mura, G. Bongiovanni, and P. Deplano, "New insights on near-infrared emitters based on Er-quinolinolate complexes: Synthesis, characterization, structural, and photophysical properties", $A d v$. Funct. Mater., vol. 17, pp. 2365-2376, 2007.

[100] L. H. Slooff, M. J. A. de Dood, A. van Blaaderen, and A. Polman, "Erbiumimplanted silica colloids with 80\% luminescence quantum efficiency", Appl. Phys. Lett., vol. 76, pp. 3682-3684, 2000.

[101] S. Lin, R. J. Feuerstein, and A. R. Mickelson, "A study of neodymiumchelate-doped optical polymer waveguides", J. Appl. Phys., vol. 79, pp.2868-2874, 1996.

[102] Y. Hasegawa, K. Murakoshi, Y. Wada, S. Yanagida, J. Kim, N. Nakashima, T. Yamanaka, "Enhancement of luminescence of $\mathrm{Nd}^{3+}$ complexes with deuterated hexafluoroacetylacetonato ligands in organic solvent", Chem. Phys. Lett., vol. 248, pp. 8-12, 1996.

[103] B. Chen, J. Xu, N. Dong, H. Liang, Q. Zhang, and M. Yin, "Spectra analysis of $\mathrm{Nd}(\mathrm{DBM})_{3}(\mathrm{TPPO})_{2}$ in MMA solution and PMMA matrix", Spectrochim. Acta Part A, vol. 60, pp. 3113-3118, 2004. 
[104] J. Lu, K. Yu, H. Wang, J. He, G. Cheng, C. Qin, J. Lin, W. Wie, and B. Peng, "Optical properties of $\mathrm{Nd}(\mathrm{TTA})_{3}(\mathrm{TPPO})_{2}$ doped polymer and its potential laser application", Opt. Mater., vol. 30, pp. 1531-1537, 2008.

[105] C. Gao, K. Cui, J. She, C. Hou, H. Guo, W. Zhao, W. Wei, and B. Peng, "Optical properties of a novel neodymium pentafluoropropionate binuclear complex", Inorganica Chim. Acta, vol. 362, pp. 2001-2005, 2009.

[106] S Ando, T Matsuura, and S Sasaki, "Perfluorinated polymers for optical waveguides", Chemtech., vol. 24, pp. 20-27, 1994.

[107] L. R. Melby, N. J. Rose, E. Abramson, and J. C. Caris, "Synthesis and fluorescence of some trivalent lanthanide complex", J. Am. Chem. Soc., vol. 86, pp. 5117-5125, 1964.

[108] F. Cui, J. Zhang, T. Cui, K. Han, B. Xie, Q. Lin, and B. Yang, "Nanoassembly of photoluminescent films containing rare earth complex nanoparticles on planar and microspherical supports", Colloid Surf. A: Physicochem. Eng. Asp., vol. 278, pp. 39-45, 2006.

[109] S. Seo, D. Zhao, K. Suh, J. H. Shin, and B. Bae, "Synthesis and luminescence properties of mesophase silica thin films doped with in-situ formed europium complex", J. Lumin., vol.128, pp. 565-572, 2008.

[110] R. Dekker, D. J. W. Klunder, A. Borreman, M. B. J. Diemeer, K. Wörhoff, A. Driessen, J. W. Stouwdam, and F. C. J. M. Veggel, "Stimulated emission and optical gain in $\mathrm{LaF}_{3}: \mathrm{Nd}$ nanoparticle-doped polymer-based waveguides", Appl. Phys. Lett., vol. 85, pp. 6104-6106, 2004.

[111] M. B. J. Diemeer, L. T. H. Hilderink, H. Kelderman, and A. Driessen, "Multimode waveguides of photodefinable epoxy for optical backplane applications", Annual Symposium IEEE/LEOS Benelux, IEEE/LEOS Benelux Chapter, Eindhoven, Nov. 30-Dec 1, pp. 53-56, 2006

[112] L. E. Sutton and O. N. Stavroudis, "Fitting refractive index data by least squares", J. Opt. Soc. Am., vol. 51, pp. 901-905, 1961.

[113] X. Han and G. Wang, "Spectral parameters of $\mathrm{Nd}^{3+}$ ion in $\mathrm{Nd}: \mathrm{KLa}\left(\mathrm{WO}_{4}\right)_{2}$ crystal", Mat. Res. Innovat., vol. 6, pp. 235-237, 2002.

[114] E. O. Serqueira, N. O. Dantas, A. F. G. Monte, and M. J. V. Bell, "Judd Ofelt calculation of quantum efficiencies and branching ratios of $\mathrm{Nd}^{3+}$ doped glasses", J. Non-Crystalline Solids, vol. 352, pp. 3628-3632, 2006.

[115] K. Kueki and Y. Koike, "Plastic optical fiber lasers and amplifiers containing lanthanide complex", Chem. Rev., vol 102, pp. 2347-2356, 2002.

[116] R. C. Powell, Physics of Solid State Laser Materials, Springer, New York, 1998.

[117] P. S. Golding, S. D. Jackson, T. A. King, and M. Pollnau, "Energy transfer processes in $\mathrm{Er}^{3+}$-doped and $\mathrm{Er}^{3+}, \mathrm{Pr}^{3+}$-codoped ZBLAN glasses", Phys. Rev. $B$, vol. 62, pp. 856-864, 2000.

[118] PhoeniX. http://www.phoenixbv.com

[119] S. Seki, T. Yamanaka, and K. Yokoyama, "Two-dimensional analysis of optical waveguides with a non-uniform finite difference method", IEE Proc.-J, vol. 138, pp.123-127, 1991.

[120] J. D. B. Bradley, M. Costa e Silva, M. Gay, L. Bramerie, A. Driessen, K. Wörhoff, J. C. Simon, and M. Pollnau, "170 GBit/s transmission in an erbium-doped waveguide amplifier on silicon", Opt. Express, vol. 17, pp. 22201-22208, 2009.

[121] V. Ostroumov, T. Jensen, J. P. Meyn, and G. Huber, "Study of luminescence concentration quenching and energy transfer upconversion in $\mathrm{Nd}$-doped 
$\mathrm{LaSc}_{3}\left(\mathrm{BO}_{3}\right)_{4}$ and $\mathrm{GdVO}_{4}$ laser crystals", J. Opt. Soc. Am. B, vol. 15, pp. 1052-1060, 1998.

[122] S. A. Payne, G. . Wilke, L. K. Smith, and W. F. Krupke, "Auger upconversion losses in Nd-doped laser glasses", Opt. Commun., vol. 111, pp. 263-268, 1994.

[123] J. L. Doualan, C. Maunier, D. Descamps, J. Landais, R. Moncorgé, "Excited-state absorption and up-conversion losses in the Nd-doped glasses for high-power lasers", Phys. Rev. B, vol. 62, pp. 4459-4463, 2000.

[124] L. C. Courrol, B. L. S. de Lima, L. R. P. Kassab, V. D. Del Cacho, S. H. Tatumi, L. Gomes, and N. U. Wetter, "Up-conversion losses in $\mathrm{Nd}^{3+}$ doped lead fluoroborate glasses", J. Non-Cryst. Solids, vol. 348, pp. 98-102, 2004.

[125] S. L. Oliveira, D. F. de Sousa, A. A. Andrade, L. A. O. Nunes, and T. Catunda, "Upconversion in $\mathrm{Nd}^{3+}$-doped glasses: Microscopic theory and spectroscopic measurements", J. Appl. Phys., vol. 103, pp. 023103-023103-8, 2008.

[126] C. L. Bonner, A. A. Anderson, R. W. Eason, D. P. Shepherd, D. S. Gill, C. Grivas, and N. A. Vainos, "Performance of a low-loss pulsed-laser-deposited $\mathrm{Nd}: \mathrm{Gd}_{3} \mathrm{Ga}_{5} \mathrm{O}_{12}$ waveguide laser at 1.06 and $0.94 \mu \mathrm{m}$ ", Opt. Lett., vol. 22, pp. 988-990, 1997.

[127] R. J. Beach, S. C. Mitchell, H. E. Meissner, O. R. Meissner, W. F. Krupke, J. M. McMahon, W. J. Bennet, D. P. Shepherd, "Continuous-wave and passively $Q$-switched cladding-pumped planar waveguide lasers", Opt. Lett., vol. 26, pp. 881-883, 2001.

[128] E. Lallier, J. P. Pocholle, M. Papuchon, Q. He, M. De Micheli, and D. B. Ostrowsky, "Integrated $\mathrm{Nd}: \mathrm{MgO}: \mathrm{LiNbO}_{3}, \mathrm{FM}$ mode-locked waveguide laser", Electron. Lett., vol. 27, pp. 936-937, 1991.

[129] N. A. Sanford, K. J. Malone, and D. R. Larson, "Extended-cavity operation of rare-earth-doped glass waveguide lasers", Opt. Lett., vol. 16, pp. 10951097, 1991.

[130] D. Findley and R. A. Clay, "The measurement of internal losses in 4-level lasers", Phys. Lett., vol. 20, pp. 277-278, 1966.

[131] W. P. Risk, "Modelling of longitudinally-pumped solid-state lasers", J. Opt. Soc. Am. B, vol. 5, pp. 1412-1421, 1988.

[132] D. Song, V. Gauss, H. Zhang, M. Gross, P. Wen, and S. Esener, "Alloptical flip-flop based on vertical cavity semiconductor optical amplifiers", Opt. Lett., vol. 32, pp. 2969-2971, 2007.

[133] S. Uhlig and M. Robertsson, "Flip chip mountable optical waveguide amplifier for optical backplane systems", Electronic Components and Technology Conference, Orlando, FL, USA, pp. 1880-1887, 2005.

[134] S. Kopetz, E. Rabe, W. J. Kang, and A. Neyer, "Polysiloxane optical waveguide layer integrated in printed circuit board", Electron. Lett., vol. 40, pp. 668-669, 2004.

[135] F. C. Krebs, "Fabrication and processing of polymer solar cells: A review of printing and coating techniques", Solar Energy Mater. \& Solar Cells, vol. 93, pp. 394-412, 2009.

[136] R. Scarmozzino, A. Gopinath, R. Pregla, and S. Helfert, "Numerical techniques for modeling guided-wave photonic device", IEEE J. Select. Topics Quantum Electron., vol.6, pp.150-162, 2000.

[137] F. Ladoucer, and J. D. Love, Silica-based buried channel waveguides and devices, Chapman \& Hall, London,1996. 
[138] C. K. Madsen and J. H. Zhao, Optical filter design and analysis: a signal processing approach, John Wiley \& Sons, New York, 1999. 


\section{Acknowledgements}

First and foremost, I thank all of my supervisors: Prof. Alfred Driessen, Prof. Markus Pollnau, Dr. Mart Diemeer, and Dr. Ay Feridun for providing me the opportunity to study and complete my Ph.D. in IOMS group. There were some personnel changes in my Ph.D. project, and without any of them this thesis would not have been possible. I would like to thank Alfred and Mart for introducing me to this group. I am thankful to Mart for his patient guidance and all instantaneous ideas for steering the polymer work in the right direction. I also thank him for his insightful suggestions and helpful discussions during the past four years even after he left from IOMS group. I would also like to thank Alfred for many stimulating discussions and wonderful ideas during my Ph.D study, as well as his close guidance and daily supervision after Mart's leave. I would like to express my gratitude to Markus and Feridun for taking over the project and my supervision after the leave of Alfred and Mart. I am grateful to Markus for his guidance and support on both polymer and $\mathrm{Al}_{2} \mathrm{O}_{3}$ based waveguide amplifiers and lasers. It was a great experience to be his student, and from whom I have learned so much about the laser physics as well as the way of doing research. I would also like to express my gratitude to my daily supervisor in the second half of my Ph.D. study, Feridun, for his assistance, patience and encouragement, which have led me constantly throughout this work.

I would also like to acknowledge the rest of my graduation committee members: Prof. Fredrik Laurell, Prof. Mein Smit, Prof. Wilem Vos, and Dr. Allard Mosk for their valuable input, time and efforts in reviewing this thesis.

During my time as a PhD-student, I have been lucky enough to have been surrounded by wonderful colleagues and I would like to acknowledge all my coworkers in IOMS for being part of an outstanding work atmosphere and for all their assistance.

I would like to express thanks to my co-workers in the Photonics Integration Technology Subgroup during the first two years of my Ph.D. study: Henry Kelderman, Lucie Hilderink, Gabriël Sengo, Murali Balakrishnan, Ronald Dekker, Edwin Klein, Gamar Hussein, Nur Ismail for all their helps. My particular appreciate goes to Henery, who picked me up at my first arrival at Twente, and introduced me the use of PM500 setup and many other things in IOMS group. And also particularly Lucie, who gave me the first guidance of polymer fabrication and made me familiar with cleanroom equipments and facilities. I also would like to thank Gabriël for helping me with the polymer fabrication as well as other cleanroom work. I would like to thank Ronald for the introduction of the loss measurement with prism coupling setup and spectrometer, the helpful discussion and suggestions on neodymium ions, and the useful small softwares he programmed, e.g. Spectraloss and ErPump. A special thanks to Edwin for the many nice conversations and invaluable help when I was learning my way. I would also like to thank Nur for helping me check the design of the mask.

I would also like to acknowledge people in the Active Device Subgroup, including Kerstin Wörhoff, Dimitri Geskus, Jonathan Bradley, Laura Agazzi, Christos Grivas, Abu Aravazhi, and Marko van Dalfsen for their help and friendship. I am very much thankful to Kerstin for the deposition of all the $\mathrm{Al}_{2} \mathrm{O}_{3}$ : $\mathrm{Nd}^{3+}$ layers, especially 
those thick layers which took her a lot of time and efforts, and her helpful suggestions. I am extremely thankful to Dimitri Geskus for his help and support with gain measurement, sample polishing, and laser experiments as well as many other optical equipments and facilities in the lab. I am also very grateful to Jon, who was always patient with my endless questions regarding $\mathrm{Al}_{2} \mathrm{O}_{3}$ fabrication, rate-equation simulations, waveguide simulation and design, lecture assistances, Ph.D. thesis, and so on. I would like to thank Laura for helping me with the lifetime measurements and loss measurements, and all the discussions about lifetime, upconversion and so on. My particular appreciation goes to Christos, the laser export from the University of South Ampton, who helped me do the polymer laser experiment during his sabbatical visit in IOMS group in 2009, and without him no polymer laser would have been possible. I am thankful to Abu for the nice conversations, and useful information regarding the job-hunting and many other things. Additional thanks to Marko for the help with gain measurement.

I would like to express my appreciation to the rest of IOMS scientific staffs: Hugo Hoekstra and René de Ridder for their assistance and ideas. My particular appreciation goes to Anton Hollin for always being friendly and helpful and giving supports on all the setups, equipments and facilities. Many thanks for seeking lasers, repairing equipments and finding solutions of measurement setups, and without him no measurement would have been possible. A special thanks to Meindert Dijkstra who did all the $\mathrm{Al}_{2} \mathrm{O}_{3}$ fabrication for me. I also thank Henk van Wolferen for his technical support on optical lenses and microscopes. I specially thank our group secretaries Rita ter Weele-Stokkers and Annitta David for their patience and assistance. I would like to thank my officemates Henri Uranus, Marcel Hoekman, Lasse Kauppinen, Fei Sun, and Fehmi Civitci for providing a cordial working atmosphere. I would like to thank Henri for sharing his studying and living experience in the Netherlands with me. A special thank to Marcel for the help and guidance with serval softwares, e.g. PhoeniX and curve loss fitting as well as many helpful discussions. I would like to thank Lasse for many nice conversations. I would also like to thank Fei and his wife Hanli for their nice dinners and friendship. I would also like to express my gratitude to other members of the IOMS group including Chaitanya Dongre, So Van Pham, Edward Bernhardi, and Imran Akca for many useful discussions, fun outings and great atmosphere. I would like to thank Edward for the help with design and ordering the Ebeam mask and useful discussions on amplifiers and lasers.

I would like to thank the people from IBM Zurich including Dr. Bert Jan Offrein, Dr. Folkert Horst and Tobias Lamprecht for the collaboration. A special thank goes to Tobias who have provided samples for us.

I would like to thank all the friends in the Netherlands for their supports and all the happiness we shared. Thanks to Xiaoqing and Haining for sharing the apartment, and particularly, Xiaoqing for having many wonderful trips together. Thanks to Wei Cheng for his delicious dinners and cakes as well as many nice conversations. I would also like to thank Yiping, Lingling, Lao Jing, Xiao Zhong, Xiao Huang, Zhang Peng, Jiwu, Xuexin, Hong Liang, and all the people who played badminton together. There was so much fun! Forgive me because I cannot put all the names here, but I would like to thank and wish you all!

Most importantly I thank my parents for their supports and encouragements, during my Ph.D. study as well as my whole life. They have given me everything they could possibly give, and there is no way of paying back to them. 


\section{List of Publications}

\section{Peer-Reviewed Journal Articles}

\section{Published or Accepted}

1. J. Yang, M. B. J. Diemeer, C. Grivas, G. Sengo, A. Driessen, and M. Pollnau, "Rare-earth-ion complex-doped fluorinated polymer for steady-state lasing", accepted by Laser Physics Letters, 2010.

2. J. Yang, K. van Dalfsen, K. Wörhoff, F. Ay, and M. Pollnau, "High-gain $\mathrm{Al}_{2} \mathrm{O}_{3}: \mathrm{Nd}^{3+}$ channel waveguide amplifiers at $880 \mathrm{~nm}, 1060 \mathrm{~nm}$ and $1330 \mathrm{~nm}$ ", accepted by Applied Physics B, 2010.

3. J. Yang, M. B. J. Diemeer, G. Sengo, M. Pollnau, and A. Driessen, "Nd-doped polymer waveguide amplifiers", accepted by IEEE Journal of Quantum Electronics, 2010.

4. J. Yang, M. B. J. Diemeer, D. Geskus, G. Sengo, M. Pollnau, and A. Driessen, "Neodymium-complex-doped photodefined polymer channel waveguide amplifiers", Optics Letters, vol. 34, pp. 473-475, 2009.

\section{Submitted}

5. C. Grivas, J. Yang, M. B. J. Diemeer, A. Driessen, and M. Pollnau, "Continuous-wave Nd-doped polymer lasers", submitted, 2010.

\section{Conferences}

\section{Oral presentations}

6. J. Yang, M. B. J. Diemeer, G. Sengo, M. Pollnau, and A. Driessen, "Characterization of Nd-doped polymer waveguide amplifiers near 1060 and 870 nm”, Conference on Lasers and Electro-Optics, San Jose, California, 2010.

7. J. Yang, F. Ay, T. Lamprecht, F. Horst, B. J. Offrein, A. Driessen, K. Wörhoff, M. Pollnau, " $\mathrm{Al}_{2} \mathrm{O}_{3}: \mathrm{Nd}^{3+}$ waveguide amplifiers for use in optical backplanes", Conference on Lasers and Electro-Optics, San Jose, California, 2010.

8. C. Grivas, J. Yang, M.B.J. Diemeer, A. Driessen, and M. Pollnau, "Continuouswave solid-state polymer laser", Conference on Lasers and Electro-Optics, San Jose, California, 2010.

9. J. Yang, C. Grivas, M.B.J. Diemeer, A. Driessen, and M. Pollnau, "Continuouswave Lasers in Polymer waveguides", 15th European Conference on Integrated Optics, Cambridge, United Kingdom, 2010. 
10. J. Yang, C. Grivas, M. B. J. Diemeer, G. Sengo, A. Driessen, and M. Pollnau, "Neodymium-complex-doped steady-state polymer waveguide lasers", in Proceedings of the Annual Symposium of the IEEE Photonics Benelux Chapter, Brussels, Belgium, pp. 61-64, 2009.

11. J. Yang, C. Grivas, M. B. J. Diemeer, G. Sengo, A. Driessen, and M. Pollnau, "Nd-complex-doped Polymer Channel Waveguide Lasers", 1st European Optical Society topical meeting on lasers, Capri, Italy, 2009.

12. J. Yang, M. B. J. Diemeer, G. Sengo, M. Pollnau, and A. Driessen, "Ndcomplex-doped Polymer Waveguide Amplifiers at 850-930 nm", Conference on Lasers and Electro-Optics Europe, Munich, Germany, 2009.

13. J. Yang, M. B. J. Diemeer, G. Sengo, M. Pollnau, and A. Driessen, "Demonstration of net gain at $1060 \mathrm{~nm}$ in a Nd-complex-doped, photo-defined polymer channel waveguide", 3rd EPS-QEOD Europhoton Conference, Paris, France, 2008.

14. J. Yang, M. B. J. Diemeer, L. T. H. Hilderink, and A. Driessen, "Experimental study of $\mathrm{Nd}(\mathrm{TTA})_{3}$ phen-doped 6-FDA/epoxy waveguides", in Proceedings Annual Workshop of the IEEE LEOS Benelux Chapter: Progress in Optical Devices and Materials, Eindhoven, The Netherlands, pp. 7-8, 2007.

\section{Post presentations}

15. J. Yang, K. van Dalfsen, K. Wörhoff, F. Ay, and M. Pollnau, "High-Gain $\mathrm{Al}_{2} \mathrm{O}_{3}: \mathrm{Nd}^{3+}$ Integrated Waveguide Amplifiers", European Materials Research Society Spring Meeting, Strasbourg, France, 2010.

16. K. van Dalfsen, J. Yang, F. Ay, K. Wörhoff, and M. Pollnau, "Nd-doped aluminum oxide integrated amplifiers at $880 \mathrm{~nm}, 1060 \mathrm{~nm}$, and $1330 \mathrm{~nm}$ ", 15th European Conference on Integrated Optics, Cambridge, United Kingdom, 2010.

17. K. van Dalfsen, J. Yang, F. Ay, K. Wörhoff, and M. Pollnau, "Neodymiumdoped $\mathrm{Al}_{2} \mathrm{O}_{3}$ Channel Waveguide Amplifiers", in Proceedings of the Annual Symposium of the IEEE Photonics Benelux Chapter, Brussels, Belgium, pp. 149152, 2009.

18. J. Yang, M. B. J. Diemeer, D. Geskus, G. Sengo, M. Pollnau, and A. Driessen, "Neodymium-complex-doped, photo-defined polymer channel waveguide amplifiers", in Proceedings of the Annual Symposium of the IEEE LEOS Benelux Chapter 2008, Enschede, The Netherlands, pp. 123-126, 2008.

19. J. Yang, M. B. J. Diemeer, L. T. H. Hilderink, M. Pollnau, and A. Driessen, "Study of the luminescence properties of $\mathrm{Nd}(\mathrm{TTA})_{3}$ phen-doped 6-FDA/epoxy waveguides", in Proceeding of SPIE, Photonics Europe, Strasbourg, France, pp. 699925-1-699925-11, 2008. 
20. J. Yang, M. B. J. Diemeer, L. T. H. Hilderink, and A. Driessen, "Luminescence study of the $\mathrm{Nd}$ (TTA) $)_{3}$ phen-doped 6-FDA/epoxy waveguide", in Proceedings of the Annual Symposium of the IEEE LEOS Benelux Chapter, Brussels, Belgium, pp. 191-194, 2007.

21. J. Yang, M. B. J. Diemeer, L. T. H. Hilderink, and A. Driessen, "Judd-Ofelt analysis of $\mathrm{Nd}$ (TTA)3phen-doped 6-FDA/epoxy planar waveguides", ePIXnet Winter School on Applications of Photonic Integration, Pontresina, Switzerland, 2007.

22. J. Yang, M. B. J. Diemeer, L. T. H. Hilderink, and A. Driessen, "Judd-Ofelt analysis of $\mathrm{Nd}(\mathrm{TTA}) 3$ phen-doped 6-FDA/epoxy planar waveguides", in Proceedings of the Annual Symposium of the IEEE LEOS Benelux Chapter 2008, Eindhoven, The Netherlands, pp. 253-256, 2006. 
